\title{
Produção de Mésons Vetoriais em Processos Foto-Induzidos no LHC
}

\author{
Bruno Duarte da Silva Moreira
}

Orientador: Prof. Dr. Fernando Silveira Navarra Coorientador: Prof. Dr. Victor Paulo Barros Gonçalves

Tese de doutorado apresentada ao Instituto de Física para a obtenção do título de Doutor em Ciências

Banca Examinadora:

Prof. Dr. Fernando Silveira Navarra (IFUSP)

Prof. Dr. Alexandre Alarcon do Passo Suaide (IFUSP)

Prof. Dr. Francisco de Oliveira Durães (MACKENZIE)

Prof. Dr. Márcio José Menon (UNICAMP)

Prof. Dr. Eduardo de Moraes Gregores (UFABC)

São Paulo 


\section{FICHA CATALOGRÁFICA \\ Preparada pelo Serviço de Biblioteca e Informação do Instituto de Física da Universidade de São Paulo}

Moreira, Bruno Duarte da Silva

Produção de mésons vetoriais em processos foto-induzidos no LHC. São Paulo, 2017.

Tese (Doutorado) - Universidade de São Paulo. Instituto de Física. Depto. de Física Experimental.

Orientador: Prof. Dr. Fernando Silveira Navarra

Área de Concentração: Física

Unitermos: 1. Colisões de íons pesados relativísticos; 2.

Cromodinâmica Quântica; 3. LHC.

USP/IF/SBI-034/2017 
Este trabalho é dedicado

À minha família 


\section{Agradecimentos}

Agradecer é uma tarefa difícil, uma vez que, em nossa caminhada, interagimos com muitas pessoas e de diversas maneiras.

Este trabalho é o resultado dessas interações.

Então, para ser justo, agradeço a todos aqueles que fazem parte da construção de quem sou e do aprimoramento que me fará ser.

Agadeço à minha família o incentivo desde cedo para o estudo, acolhendo meus momentos incondicionalmente e o apoio à minha vinda para a cidade de São Paulo. Em particular agradeço à minha mãe Cláudia TUDO (e aqui, tudo é muito mais do que essa palavra parece significar) aquilo que tem feito por mim e por ter estado tão próxima, mesmo estando longe, durante o doutorado. Agradeço ao meu pai Onelly, o acompanhamento tão de perto nos meus primeiros anos de escola e pelo companheirismo no período do doutorado. Agradeço, também, à minha irmã Helen, ser minha melhor amiga e ter trazido ao mundo meu afilhado Pedro que, sem dúvida, foi o maior presente que essa família já ganhou e a maior motivação para os próximos passos. E agradeço à minha madrinha Daniela ser tão carinhosa comigo e ser um grande exemplo de determinação, o qual procuro seguir diariamente.

Agradeço ao meu orientador Dr. Fernando Silveira Navarra, a orientação e a amizade. Agradeço, também, ter me proporcionado um ambiente de trabalho maravilhoso e tranquilo, fazendo com que eu me sentisse em casa muito rapidamente ao chegar em São Paulo .

Agradeço ao coorientador Dr. Victor Paulo Barros Gonçalves, a amizade que já dura muito tempo e os conhecimentos compartilhados com tanta generosidade. Agradeço também por ter sido, juntamente à minha família, a pessoa que me incentivou para de Pelotas chegar a São Paulo.

Aos colegas, professores e funcionários do grupo de hádrons e física teórica a agradável convivência nesses últimos anos e, principalmente, a amizade.

Aos colegas de república, a amizade e parceria durantes este período. Vocês sempre serão lembrados como a minha família de São Paulo.

Agradeço à professora Dra. Fabiana Carvalho e ao professor Dr. Carlos Bertulani a amizade e as colaborações durante o período do doutorado.

Ao pessoal da CPG que me atendeu com muita dedicação e paciência desde o primeiro dia de doutorado. 
Aos professores e alunos da Universidade Federal de Pelotas, onde realizei meus estágios com o professor Victor, agradeço a recepção e a estrutura cedida em minhas visitas.

Agradeço aos meus amigos de Pelotas, especialmente aos da UFPel, com os quais pude vivenciar, com muita alegria, inúmeras experiências, durante minhas visitas ao RS. Fizeram bastante falta nesses anos.

Aos professores da USP e da UFPel que participaram, de forma concreta, da minha formação.

Agradeço, por fim, à Coordenação de Aperfeiçoamento de Pessoal de Nível Superior, a bolsa durante o tempo do doutorado. 


\section{Resumo}

O advento dos colisores modernos tem proporcionado novas possibilidades de estudo em física de partículas como, por exemplo, a busca por nova física e o estudo de novos estados da matéria hadrônica. Em particular, em colisões de íons pesados em altas energias, acredita-se que um novo estado da matéria seja formado antes da colisão, o chamado condensado de vidros de cor. Tal estado seria a condição inicial de um plasma de quarks e glúons e é caracterizado por altas densidades de pártons (quarks e glúons). De fato, as equações de evolução da Cromodinâmica Quântica predizem que, para virtualidades moderadas e altas energias, os hádrons se tornam sistemas extremamente densos devido ao crescimento das distribuições de glúons nessas condições. Um crescimento indefinido das distribuições de glúons poderia levar à violação do vínculo de unitariedade. Para evitar essa violação, existe um mecanismo chamado de saturação de pártons o qual contém o crescimento das distribuições de quarks e glúons de forma a respeitar o vínculo de unitariedade. Teoricamente, no limite de altas energias, observa-se que os hádrons são populados especialmente por glúons. Experimentalmente, existem indícios da saturação em colisões ep, $p p, p A$ e $A A$, contudo, isso ainda é uma questão em aberto. Portanto, no limite de altas densidades, espera-se que a física não linear, a qual leva em conta efeitos de recombinações de glúons, passe a se manifestar. Uma alternativa para o estudo da saturação em colisores hadrônicos são os processos foto-induzidos, os quais ocorrem em interações ultraperiféricas. Em uma colisão ultraperiférica entre dois hádrons o parâmetro de impacto é maior do que a soma dos raios dos hádrons, de forma que interações fortes são suprimidas. Dessa forma os hádrons atuam como fontes de fótons (quase reais) podendo ocorrer interações fóton-hádron e fóton-fóton. Nesse trabalho estudamos a fotoprodução difrativa de mésons vetoriais em energias do LHC para colisões ultraperiféricas prótonpróton, próton-núcleo e núcleo-núcleo e a produção dupla de mésons vetoriais em colisões fóton-fóton e devido ao mecanismo de duplo espalhamento $\gamma p(A)$. Mostramos como esses processos podem ser usados para estudar a física de altas energias e os efeitos de saturação. Os efeitos de saturação em nossos cálculos são levados em conta através do formalismo de dipolo de cor, que é uma das ferramentas básicas usadas nessa tese. O tratamento de colisões ultraperiféricas hádron-hádron foi feito com a aproximação de fótons equivalentes que assim como o formalismo de dipolo, foi extensamente usada. Nossos resultados apontam que o estudo fenomenológico e experimental dos tipos de processos citados acima são factíveis e podem ser usados para vincular a dinâmica da QCD em altas energias.

Palavras-chave: colisões ultraperiféricas, LHC, mésons vetoriais, QCD e saturação. 


\section{Abstract}

The advent of the modern colliders has provided new possibilities of study in particle physics as, for example, the search for new physics and the study of new states of the hadronic matter. In particular, in high energy heavy ion collisions is expected that a new state can be formed before the collision, the so called Color Glass Condensate. This state would be the initial condition of the Quark Gluon Plasma and is characterized by high parton (quarks and gluons) densities. Indeed, the Quantum Chromodynamics evolution equations predict that, for moderate virtualities and high energies, the hadrons become extremely dense systems due to the growth of the parton distribution in these conditions. A indefinite growth of the parton distributions could lead to the violation of the unitarity bound. To avoid this violation, there is a mechanism called parton saturation which tames the growth of the parton distributions to satisfy the unitarity bound. Theoretically, in the high energy limit, one can see that the hadrons are populated mainly by gluons. Experimentally, there are indications of the saturation in ep, $p p, p A$ and $A A$ collisions, however, this is an open question. So, in the high density limit, we expect that the nonlinear physics, which takes into account gluon recombination effects start to manifest itself. An alternative for the study of the saturation at hadronic colliders are the photon-induced processes, which occurs in ultraperipheral collisions. In a ultraperipheral collision between two hadrons the impact parameter is greater than the sum of the radii of the hadrons, so the strong interaction is suppressed. Thus, the hadrons act as sources of (almost real) photons and may occur photon-hadron and photon-photon interactions. In this work we study the diffractive photoproduction of vector mesons at LHC and future colliders energies in ultraperipheral proton-proton, proton-nucleus and nucleus-nucleus collisions and the double vector meson production in photon-photon collisions and due to the double $\gamma p(A)$ scattering mechanism. We show how these processes can be used to study the high energy physics and the saturation effects. These effects are considered in our calculations through the color dipole formalism, which is one of the basic tools used in this thesis. The treatment of the ultraperipheral hadron-hadron collisions was done through the equivalent photon approximation that just as in the dipole formalism, was widely used. Our results point that the phenomenological and experimental studies of the processes cited above are feasible and can be used to constraint the QCD dynamics in high energies.

Keywords: LHC, QCD, saturation, ultraperipheral collisions and vector mesons. 


\section{Publicações}

Durante o período em que o doutorado foi realizado, os seguintes trabalhos foram submetidos à publicação:

1. V. P. Gonçalves, B. D. Moreira and F. S. Navarra, Investigation of diffractive photoproduction of $J / \Psi$ in hadronic collisions, Phys. Rev. C 90, no. 1, 015203 (2014).

2. V. P. Gonçalves, B. D. Moreira and F. S. Navarra, Exclusive $\Upsilon$ photoproduction in hadronic collisions at CERN LHC energies, Phys. Lett. B 742, 172 (2015).

3. F. Carvalho, V. P. Gonçalves, B. D. Moreira and F. S. Navarra, Double vector meson production in the International Linear Collider, Eur. Phys. J. C 75, no. 8, 392 (2015).

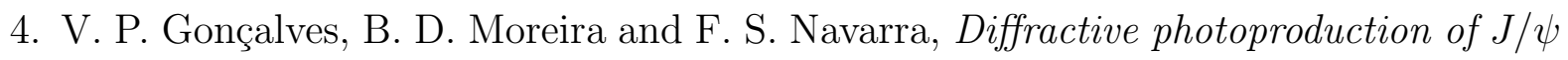
at LHC energies, J. Phys. Conf. Ser. 630, no. 1, 012061 (2015).

5. V. P. Gonçalves, B. D. Moreira and F. S. Navarra, Double vector meson production in $\gamma \gamma$ interactions at hadronic colliders, Eur. Phys. J. C 76, no. 3, 103 (2016).

6. V. P. Gonçalves, B. D. Moreira and F. S. Navarra, Double vector meson production in photon - hadron interactions at hadronic colliders, Eur. Phys. J. C 76, no. 7, 388 (2016).

7. V. P. Gonçalves, B. D. Moreira, F. S. Navarra and D. Spiering, Exclusive vector meson production with a leading neutron in photon - hadron interactions at hadronic colliders, Phys. Rev. D 94, no. 1, 014009 (2016).

8. B. D. Moreira, C. A. Bertulani, V. P. Gonçalves and F. S. Navarra, Production of exotic charmonium in $\gamma \gamma$ interactions at hadron colliders, Phys. Rev. D 94, no. 9, 094024 (2016).

9. V. Goncalves, B. D. Moreira and F. Navarra, Vector Meson Production in Photon induced Interactions at the LHC, PoS LHCP 2016, 228 (2016).

10. V.P. Goncalves, B. D. Moreira and F. S. Navarra, Exclusive heavy vector meson photoproduction in hadronic collisions at the LHC: predictions of the Color Glass Condensate model for Run 2 energies, Phys. Rev. D 95, no. 5, 054011 (2017).

11. C. A. Bertulani, V. P. Gonçalves, B. D. Moreira and F. S. Navarra, Production of exotic charmonium in ultra-peripheral heavy ion collisions, EPJ Web Conf. 137, 06019 (2017). 
Nessa tese, vamos discutir os trabalhos presentes nas referências 1, 2, 3, 5, 6 e 10 . 


\section{Índice}

1. Introdução . . . . . . . . . . . . . . . . . . . . . . . . 2

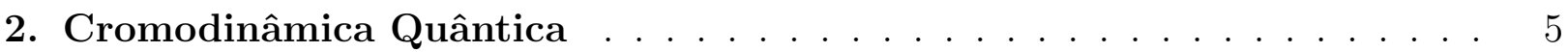

2.1 A Lagragiana da QCD e a Invariância de Gauge . . . . . . . . . . . . . . 6

2.2 A Constante de Acoplamento da QCD e a Liberdade Assintótica . . . . . . . 9

2.3 O Espalhamento Profundamente Inelástico . . . . . . . . . . . . . . . . 11

2.3 .1 Cinemática do DIS . . . . . . . . . . . . . . . . . . . . 12

2.3.2 Espalhamento elástico elétron-quark . . . . . . . . . . . . . . 13

2.3.3 Espalhamento inelástico elétron - hádron . . . . . . . . . . . 15

2.4 A violação do scaling de Bjorken e as equações DGLAP . . . . . . . . . . . . 17

2.5 Análise global e obtenção das PDFs . . . . . . . . . . . . . . . . . . . . 21

2.6 Conclusão . . . . . . . . . . . . . . . . . . . . . . . . . 22

3. Dinâmica de pequeno $\mathrm{x} \ldots \ldots \ldots \ldots \ldots \ldots$

3.1 Formalismo de Dipolo . . . . . . . . . . . . . . . . . . . . . . . 23

3.2 Solução das equações DGLAP no limite de duplo logaritmo dominante . . 26

3.3 Equação BFKL . . . . . . . . . . . . . . . . . . . . . . . . . . . . 28

3.4 Limite de Froissart-Martin . . . . . . . . . . . . . . . . . . . . . . . . 30

3.5 Equação GLR e efeitos de saturação . . . . . . . . . . . . . . . . . . . . 31

3.6 Saturação no formalismo de dipolo de cor . . . . . . . . . . . . . . . . . 33

3.7 Equação $\mathrm{BK} \ldots \ldots$. . . . . . . . . . . . . . . . . . . . . . . . . . . . 34

3.8 Modelos fenomenológicos para $\mathcal{N} \ldots \ldots \ldots \ldots$

3.8 .1 Modelo GBW . . . . . . . . . . . . . . . . . . . . . . . 37

3.8 .2 Modelo IIM . . . . . . . . . . . . . . . . . . . . . . . . . . . 38

3.8 .3 Modelo bCGC . . . . . . . . . . . . . . . . . . . . . . . . . . . . . 39

3.8.4 Modelo rcBK . . . . . . . . . . . . . . . . . . . . . . . . . . . 39

3.9 Evidências experimentais da saturação $\ldots \ldots$. . . . . . . . . . . . . . . 40

3.10 Conclusão . . . . . . . . . . . . . . . . . . . . . . . . . . . . . 42

4. Processos foto-induzidos em colisões hadrônicas . . . . . . . . . . . . . . 44

4.1 O método de fótons equivalentes . . . . . . . . . . . . . . . . . 46

4.2 Colisões ultraperiféricas em colisores hadrônicos . . . . . . . . . . . . . . 48

4.2.1 Fluxo de fótons independente do parâmetro de impacto . . . . . . . . 48

4.2.2 Fluxo de fótons dependente do parâmetro de impacto . . . . . . . . . 49

4.3 Produção exclusiva de mésons vetoriais em colisões ultraperiféricas . . . . . . . 52

4.4 Produção de partículas em processos foto-induzidos no LHC . . . . . . . . 55 
4.5 Conclusão . . . . . . . . . . . . . . . . . . . . . 56

5. Fotoprodução difrativa de mésons vetoriais em interações $\gamma h \ldots \ldots$

5.1 Formalismo de dipolo para a produção quase elástica de mésons vetoriais . . 59

5.1.1 Formalismo de dipolo para a produção difrativa de mésons vetoriais

em colisões $\gamma A$. . . . . . . . . . . . . . . . . . . . . . . . . . . . . . . . . . . 62

5.2 Fotoprodução difrativa de $J / \psi$ em colisões ultraperiféricas . . . . . . . . . . 63

5.2 .1 Colisões $\gamma p$ e $\gamma P b \ldots \ldots \ldots \ldots$. . . . . . . . . . . . . 64

5.2 .2 Colisões $\mathrm{pp}, \mathrm{pPb}$ e $\mathrm{PbPb} \ldots \ldots \ldots \ldots \ldots \ldots$

5.2.3 Fotoprodução difrativa incoerente de $J / \psi$ em colisões $\mathrm{PbPb}$. . . . . 68

5.3 Fotoprodução difrativa de $\Upsilon$ em colisões ultraperiféricas . . . . . . . . . . . . 70

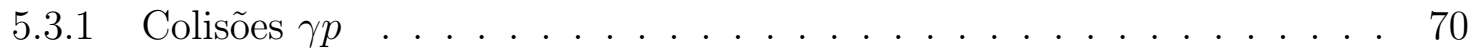

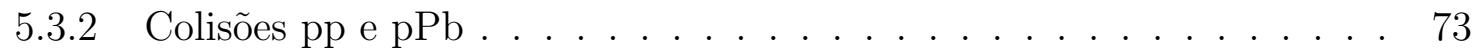

5.4 Previsões para o Run II do LHC . . . . . . . . . . . . . . . . . . . . . . 75

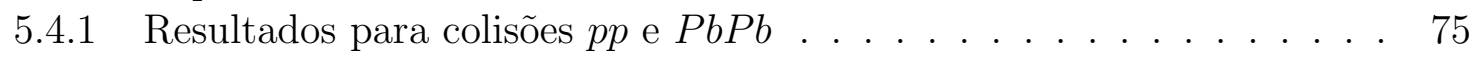

5.5 Conclusões . . . . . . . . . . . . . . . . . . . . 78

6. Produção dupla de mésons vetoriais em colisões $\gamma \gamma$. . . . . . . . . . . . 80

6.1 Formalismo de dipolo para a produção dupla de mésons vetoriais . . . . . . . 80

6.1 .1 Previsões para o ILC . . . . . . . . . . . . . . . . . . 82

6.2 Previsões para o LHC e o FCC . . . . . . . . . . . . . . . . . . . . . . . . 88

6.3 Conclusões . . . . . . . . . . . . . . . . . . . . . . . 94

7. Produção dupla de mésons vetoriais em interações $\gamma h$ no LHC . . . . . . 96

7.1 O mecanismo de duplo espalhamento . . . . . . . . . . . . . . . 96

7.2 Previsões para o LHC . . . . . . . . . . . . . . . . . . . . . . . . . . . . . . . . . . . . . . . . . . .

7.3 Conclusões . . . . . . . . . . . . . . . . . . . . . . . . . 100

8. Conclusão . . . . . . . . . . . . . . . . . . . . . . . . . 101

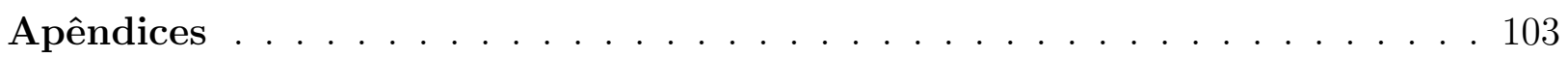

A. Funções de onda do cone de luz . . . . . . . . . . . . . . . . . . . . . 104

A.1 Função de onda do fóton . . . . . . . . . . . . . . . . . . . . . . . . . . 104

A.1.1 Polarização longitudinal . . . . . . . . . . . . . . . . . . . . . . . . . . . . . . . . . . . . . . . . . . . . . .

A.1.2 Polarização transversal . . . . . . . . . . . . . . . . . . . 107

A.2 Funções de onda de mésons vetoriais . . . . . . . . . . . . . . . . . 111

B. Soluções da Equação BK . . . . . . . . . . . . . . . . . . . . . . . . 114

B.1 Soluções fora da região de saturação . . . . . . . . . . . . . . . . . . . . . . . 114

B.1.1 Aproximação de Difusão . . . . . . . . . . . . . . . . . . . . . . . . 114

B.1.2 Aproximação de Duplo Logaritmo Dominante . . . . . . . . . . . . . 116

B.1.3 Solução próximo ao regime de saturação . . . . . . . . . . . . . . . . 117

B.2 Solução dentro da região de saturação . . . . . . . . . . . . . . . . . . . . . 119 
C. Aproximação de Fótons Equivalentes . . . . . . . . . . . . . . . . . . 121

C.1 Derivação do espectro de fótons equivalentes . . . . . . . . . . . . . . . . 121

C.2 Espectro de Fótons Equivalentes Dependente do Parâmetro de Impacto . . . 126

C.2.1 Fatores de Forma . . . . . . . . . . . . . . . . . . . . 128 


\section{Relação de Figuras}

2.1 Propagadores da QCD . . . . . . . . . . . . . . . . . . . . 8

2.2 Vértices da QCD. . . . . . . . . . . . . . . . . . . . . . . . . 9

2.3 Espalhamento profundamente inelástico elétron - próton. . . . . . . . . . 12

2.4 Espalhamento elétron - párton. . . . . . . . . . . . . . . . 14

2.5 Função de estrutura $F_{2}$ em função da virtualidade. . . . . . . . . . . . 18

2.6 Diagramas que contribuem em ordem $\alpha_{s}$ para o espalhamento profundamente inelástico. . . . . . . . . . . . . . . . . . . . . . 19

2.7 PDFs extraídas de dados experimentais. . . . . . . . . . . . . . . 21

3.1 Amplitude de espalhamento elástico $\gamma^{*} p \rightarrow \gamma^{*} p$ via formalismo de dipolo. . . 24

3.2 Amplitude elástica para o espalhamento entre um fóton virtual e um próton em altas energias. . . . . . . . . . . . . . . . . 28

3.3 Diagrama $\left(x, Q^{2}\right)$ para a QCD em altas energias. Aqui $Q_{s}$ é a escala de saturação. . . . . . . . . . . . . . . . . . . . 33

3.4 Dipolo de cor inicial e depois de um passo de evolução em rapidez. . . . . . 35

3.5 Cascata de glúons no limite de grande $N_{c} \ldots \ldots$. . . . . . . . . . 36

3.6 Scaling geométrico observado na seção de choque $\sigma^{\gamma^{*} p}$ (à esquerda) e razão

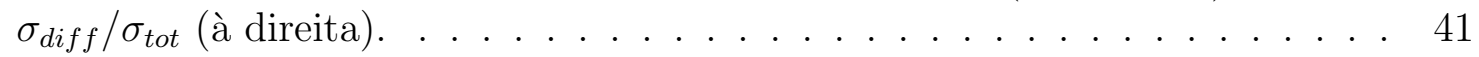

3.7 Dependência da multiplicidade hadrônica com a rapidez em colisões $A A$ no RHIC (à esquerda) e dependência da multiplicidade hadrônica com a energia em colisões $p p$ e $A A$ para energias do RHIC e do LHC (à direita). . . . . . . 42

3.8 Dependência de $\left\langle p_{T}\right\rangle$ com $\sqrt{s} \ldots \ldots \ldots \ldots \ldots$

4.1 Campo eletromagnético de uma carga para $v=0$ (à esquerda) e $v \sim c$ (à

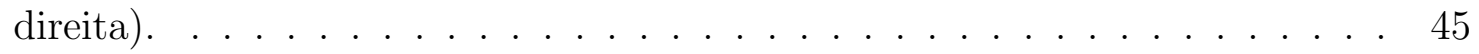

4.2 Fluxo de fótons equivalentes associados ao próton. . . . . . . . . . . . . 49

4.3 Processo $\gamma \gamma$ em interações hadrônicas. . . . . . . . . . . . . . . . 50

4.4 Comparação entre os fluxos de fótons dependentes de $b \ldots \ldots \ldots$. . . . . 51

4.5 Dependência das seções de choque de produção de mésons vetoriais com a energia de centro de massa $\gamma p$ comparadas com dados de HERA. . . . . . . 53

4.6 Distribuição de rapidez para a fotoprodução de $\rho$ em colisões ultraperiféricas AuAu no RHIC. . . . . . . . . . . . . . . . . . . . . . . . . . . 54

4.7 Distribuições de $p_{T}$ para a produção de $\mu^{+} \mu^{-}$(painel esquerdo) e de $\mu^{ \pm} e^{\mp}$ (painel direito) em colisões $p p$ com $\sqrt{s}=7 \mathrm{TeV}$ em interações $\gamma \gamma$. Dados da colaboração CMS. . . . . . . . . . . . . . . . . . . . 56 
4.8 Distribuições de rapidez para a produção de $J / \psi$ (painel esquerdo) e de $\rho$ (painel direito) em colisões ultraperiféricas $\mathrm{PbPb} \mathrm{com} \sqrt{s}=2.76 \mathrm{TeV}$. Dados das colaborações ALICE e CMS. . . . . . . . . . . . . . . . 57

5.1 Cinemática do DIS difrativo. . . . . . . . . . . . . . . . . . . . . . 59

5.2 Amplitude de espalhamento quase elástica $\gamma^{*} p \rightarrow V p \ldots \ldots$. . . . . . . . 60

5.3 Seção de choque $\gamma p(P b)$ como função da energia do centro de massa $\gamma p(P b)$. À esquerda: processo $\gamma^{*} p \rightarrow J / \psi p$. À direita: $\gamma^{*} P b \rightarrow J / \psi P b$ (coerente). . . 64

5.4 Distribuição de rapidez para a fotoprodução difrativa de $J / \psi$ em colisões $p p$ em $\sqrt{s}=7 \mathrm{TeV}$ (à esquerda) e $\sqrt{s}=14 \mathrm{TeV}$ (à direita). . . . . . . . . . .

5.5 Distribuição de rapidez de $J / \psi$ 's criados através de fotoprodução difrativa em colisões $\mathrm{PbPb}$ a $\sqrt{s}=2,76 \mathrm{TeV}$ (à esquerda) e a $\sqrt{s}=5,5 \mathrm{TeV}$ (à direita). 67

5.6 Distribuição de rapidez para a fotoprodução difrativa de $J / \psi$ em colisões $p P b$ em $\sqrt{s}=5 \mathrm{TeV} \ldots \ldots \ldots \ldots \ldots \ldots$

5.7 Distribuição de rapidez para a fotoprodução difrativa incoerente de $J / \psi$ em colisões $\mathrm{PbPb}$ em $\sqrt{s}=2,76 \mathrm{TeV}$. À esquerda usamos o modelo Gaus-LC para a função de onda do méson e à direita, o modelo Boosted Gaussian. . .

5.8 Distribuição de rapidez para a fotoprodução difrativa incoerente de $J / \psi$ em colisões $\mathrm{PbPb}$ em $\sqrt{s}=5,5 \mathrm{TeV}$. À esquerda usamos o modelo Gaus-LC para a função de onda do méson e à direita, o modelo Boosted Gaussian. . . . . . .

5.9 Comparação da parte transversal da função de onda do $\Upsilon$ para os modelos Boosted Gaussian e Gaus-LC. . . . . . . . . . . . . . . . . . . . . . . . . 71

5.10 Seção de choque de fotoprodução de $\Upsilon$ em colisões $\gamma p$. . . . . . . . . . . . 72

5.11 Distribuição de rapidez para a fotoprodução difrativa de $\Upsilon$ em colisões $p p$ a $\sqrt{s}=7 \mathrm{TeV}$ (acima) e a $\sqrt{s}=14 \mathrm{TeV}$ (abaixo). ’̀ esquerda usamos o modelo Boosted Gaussian para a função de onda do méson e à direita, o modelo Gaus-LC. . . . . . . . . . . . . . . . . . . .

5.12 Distribuição de rapidez para a fotoprodução difrativa de $\Upsilon$ em colisões $p P b$ em $\sqrt{s}=5 \mathrm{TeV}$. À esquerda usamos o modelo Boosted Gaussian para a função de onda do méson e à direita, o modelo Gaus-LC. . . . . . . . . . . . .

5.13 Contribuição transversal do overlap entre as funções de onda do fóton e do méson vetorial integrados em $z$ para $Q^{2}=0$. . . . . . . . . . . . . 76

5.14 Distribuições de rapidez em colisões $p p$ a $\sqrt{s}=13 \mathrm{TeV} \ldots \ldots$. . . . . . . . . 77

5.15 Distribuições de rapidez em colisões $\mathrm{PbPb}$ a $\sqrt{s}=5.02 \mathrm{TeV}$. . . . . . . . . 78

6.1 Produção dupla de mésons vetoriais em interações $\gamma^{*} \gamma^{*}$ em altas energias na representação de dipolos de cor. . . . . . . . . . . . . . . . . . . . 81

6.2 Dependência do produto $B_{V_{1} V_{2}} \sigma\left(\gamma^{*} \gamma^{*} \rightarrow V_{1} V_{2}\right)$ com a energia assumindo $V_{1}=V_{2}$ e considerando $Q_{1}^{2}=Q_{2}^{2}=0 \ldots \ldots \ldots$. . . . . . . 83

6.3 Dependência do produto $B_{V_{1} V_{2}} \sigma\left(\gamma^{*} \gamma^{*} \rightarrow V_{1} V_{2}\right)$ com a energia assumindo $V_{1} \neq V_{2}$ e considerando $Q_{1}^{2}=Q_{2}^{2}=0$. . . . . . . . . . . . 84

6.4 Dependência do produto $B_{V_{1} V_{2}} \sigma\left(\gamma^{*} \gamma^{*} \rightarrow V_{1} V_{2}\right)$ com a virtualidade assumindo $V_{1}=V_{2}$ e considerando $Q_{1}^{2}=Q_{2}^{2}=Q^{2}$ para $W=500 \mathrm{GeV} \ldots \ldots$. . . . . 85

6.5 Dependência do produto $B_{V_{1} V_{2}} \sigma\left(\gamma^{*} \gamma^{*} \rightarrow V_{1} V_{2}\right)$ com a virtualidade assumindo $V_{1} \neq V_{2}$ e considerando $Q_{1}^{2}=Q_{2}^{2}=Q^{2}$ para $W=500 \mathrm{GeV} \ldots \ldots$. . . . 86 
6.6 Dependência da seção de choque normalizada com a energia e com a virtualidade $\left(Q_{1}^{2}=Q_{2}^{2}=Q^{2}\right) \ldots \ldots \ldots \ldots \ldots \ldots$. . . . . . . . . . . . . . . . .

6.7 Dependência de $\sigma\left(\gamma^{*} \gamma^{*} \rightarrow V_{1} V_{2}\right)$ com a energia para diferentes estados finais, considerando $Q_{1}^{2}=Q_{2}^{2}=0 \ldots \ldots \ldots$. . . . . . . . . 87

6.8 Comparação entre as partes linear e completa do modelo IIM-S para o comportamento

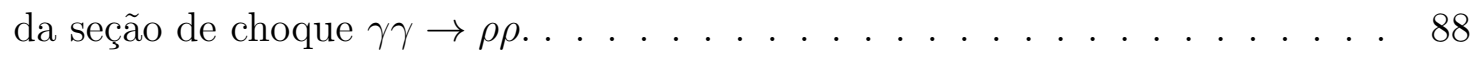

6.9 Diagrama para o processo $A A \rightarrow A V V A$ devido à interação $\gamma \gamma$. . . . . . . 89

6.10 Distribuições de rapidez para a produção dupla de mésons vetoriais em colisões

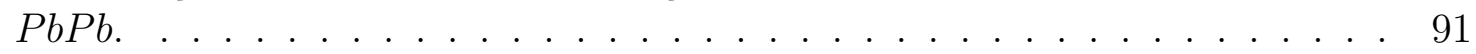

6.11 Distribuições de rapidez para a produção dupla de mésons vetoriais em colisões

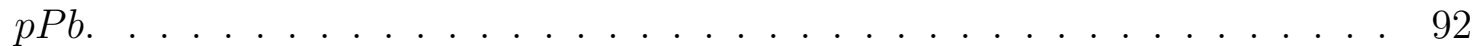

6.12 Distribuições de rapidez na produção dupla de mésons vetoriais em colisões $p p .93$

6.13 Comportamento das seções de choque com $\sqrt{s}$. . . . . . . . . . . . . . . 94

7.1 Produção dupla de mésons vetoriais através do duplo espalhamento $\gamma h$. . . . 97

7.2 Dependência da seção de choque com a energia para a produção dupla de mésons vetoriais através dos mecanismos de duplo espalhamento e de interação $\gamma \gamma \ldots \ldots \ldots \ldots \ldots \ldots \ldots$

7.3 Distribuições de rapidez para a produção dupla de $\rho$ (primeira coluna), $J / \psi$ (segunda coluna) e para a produção mista $\rho J / \psi$ (terceira coluna) devido ao mecanismo de duplo espalhamento em colisões $\mathrm{PbPb}$ a $\sqrt{s}=5.5 \mathrm{TeV}$ (painel superior) e em colisões $p P b$ a $\sqrt{s}=5 \mathrm{TeV}$ (painel inferior). As distribuições de rapidez estão em mb. . . . . . . . . . . . . . . . . . . . . . . . . . 99

A.1 Diagrama para a função de onda do cone de luz do fóton. . . . . . . . . . . . 105

B.1 Comportamento da função característica da BFKL. . . . . . . . . . . . . . 116

C.1 (a) Colisão entre um fóton real e uma partícula de quadrimomentum $q$ e (b) uma partícula de quadrimomentum inicial $p$ emite um fóton virtual que interage com uma partícula de quadrimomentum $q$. Nos dois casos é gerado um estado final com quadrimomentum $Q . \ldots$. . . . . . . . . . . . . 122 


\section{Relação de Tabelas}

3.1 Parâmetros do modelo GBW. . . . . . . . . . . . . . . . . . . . . . 38

3.2 Parâmetros do modelo IIM. . . . . . . . . . . . . . . . . . . . . . . . . . . . . . 39

3.3 Parâmetros do modelo bCGC . . . . . . . . . . . . . . . . . . . 39

4.1 Parâmetros e limites cinemáticos para processos fóton-hádron e fóton-fóton no RHIC e no LHC. . . . . . . . . . . . . . . . . . . . . 46

5.1 Seção de choque total para a produção difrativa de $J / \psi$ em colisões $p p, p P b$ e $\mathrm{PbPb}$ em energias do LHC. . . . . . . . . . . . . . . . . . . . . . . . 68

5.2 Seções de choque totais de fotoprodução exclusiva de $\Upsilon$ em colisões $p p$ em $\sqrt{s}=7,8$ e $14 \mathrm{TeV}$ e colisões $p P b$ em $\sqrt{s}=5 \mathrm{TeV}$ considerando os modelos Gaus-LC e Boosted Gaussian para a função de onda do $\Upsilon$. . . . . . . . . . . 75

5.3 Seções de choque totais de fotoprodução exclusiva de mésons vetoriais em colisões $p p$ em $\sqrt{s}=13 \mathrm{TeV}$ e colisões $\mathrm{PbPb}$ em $\sqrt{s}=5,02 \mathrm{TeV} \ldots \ldots$. . . . 79

6.1 Seções de choque totais para a produção dupla de $\rho$ em interações $\gamma \gamma$ em colisões $p p, p P b$ and $\mathrm{PbPb}$ nas energias do RHIC, LHC, FCC e CEPC SPPC. Valores em nb. . . . . . . . . . . . . . . . . . . . . 94

6.2 Seções de choque totais para a produção dupla de $J / \psi$ em interações $\gamma \gamma$ em colisões $p p, p P b$ and $\mathrm{PbPb}$ nas energias do RHIC, LHC, FCC e CEPC SPPC. Valores em pb. . . . . . . . . . . . . . . . . . . . 95

7.1 Seções de choque totais para a produção dupla de mésons vetoriais através dos mecanismos de duplo espalhamento e de interação $\gamma \gamma$ em energias do LHC. 98

A.1 Parâmetros para a função de onda Gaus-LC. . . . . . . . . . . . . . . . . . . 112

A.2 Parâmetros para a função de onda Boosted Gaussian. . . . . . . . . . . . . . 112 


\section{Capítulo 1}

\section{Introdução}

A Cromodinâmica Quântica ("Quantum Chromodynamics" - QCD) é a teoria que estuda as interações entre quarks e glúons (coletivamente denominados de pártons). Quarks e glúons não são observados como partículas livres na natureza e em vez disso formam os hádrons (como prótons, nêutrons, píons e etc). Nesse trabalho estudamos a QCD através de colisões de hádrons no regime de altas energias. Em colisões lépton-hádron e hádron-hádron com momentum transferido fixo (virtualidade fixa) e altas energias, espera-se um rápido crescimento da distribuição de glúons dentro dos hádrons, conforme descrito pelas equações de evolução lineares da QCD DGLAP (devida à Dokshitzer, Gribov, Lipatov, Altarelli e Parisi) e BFKL (devida à Balitsky, Fadin, Kuraev e Lipatov), tornando o hádron um sistema denso. Por outro lado um crescimento rápido das distribuições de glúons com a energia, tal como o descrito pelas equações lineares da QCD, viola o vínculo de unitariedade da matriz de espalhamento (matriz $S$ ). O mecanismo responsável por diminuir a velocidade de crescimento das distribuições de glúons é chamado de saturação de glúons. Esse mecanismo está associado a efeitos de recombinação de glúons (processos do tipo $g g \rightarrow g$ ) que são descritos pelas equações de evolução não lineares da QCD.

Atualmente, a física de partículas passa por um importante período devido à possibilidade de estudos experimentais em condições extremas nos grandes aceleradores de partículas. Em particular, o LHC ("Large Hadron Collider") possibilita o estudo de colisões hadrônicas em energias de centro de massa $(\sqrt{s})$ jamais alcançadas em outros aceleradores. A região cinemática provada pelo LHC motiva a busca/estudo por novos estados da matéria, dentre eles o condensado de vidro de cor ("Color Glass Condensate" - CGC) e o plasma de quarks e glúons ("Quark Gluon Plasma" - QGP). Nesse trabalho, estamos interessados na física do CGC, que seria o estado de alta densidade partônica onde os hádrons se encontram antes da colisão enquanto o QGP é o estado que pode ocorrer após a colisão, onde quarks e glúons estariam misturados e livres imediatamente após a colisão formando um plasma.

Uma forma bastante adequada de se estudar a estrutura hadrônica em altas energias, é através do espalhamento profundamente inelástico elétron-hádron ("Deep Inelastic Scattering" - DIS), onde apenas uma das partículas possui estrutura interna. O estudo experimental do DIS em altas energias será possível quando os novos colisores elétron-hádron entrarem em funcionamento. Por outro lado, uma alternativa para o estudo de efeitos de altas densidades é encontrada em colisões ultraperiféricas entre dois hádrons, que é um tipo de colisão onde o parâmetro de impacto dos hádrons é maior que a soma de seus raios. Em uma colisão desse tipo, prótons e núcleos ultra-relativísticos atuam como fontes de fótons que interagem com o 
alvo. Nesse caso, podemos estudar a interação hádron-hádron através do método de fótons equivalentes, onde essa interação pode ser tratada em termos das interações fóton-hádron e/ou fóton-fóton. Estudamos as interações fóton-hádron e fóton-fóton, pelo formalismo de dipolo de cor, que é uma poderosa ferramenta para o estudo de espalhamentos em altas energias, além de ser capaz de incorporar efeitos de saturação. No formalismo de dipolo, um fóton virtual flutua num par quark-antiquark (dipolo de cor) o qual interage com o hádron alvo. Esse formalismo nos permite estudar o processo fóton-hádron, em termos do processo dipolo-hádron, sendo esse último o processo onde efeitos de saturação são modelados. Em nosso trabalho, para o caso da interação fóton-fóton, estudamos o caso onde os dois fótons flutuam em dipolos de cor, levando ao espalhamento dipolo-dipolo.

Na presente tese, estudamos a fotoprodução de mésons vetoriais através do método de fótons equivalentes e do formalismo de dipolo e mostramos como tal produção pode ser usada para estudar a dinâmica da QCD em altas energias. Mais precisamente, estudamos a produção de um único méson vetorial em colisões hádron-hádron (através do subprocesso fóton-hádron) e a dupla produção de mésons vetoriais através de colisões fóton-fóton e da dupla colisão fóton-hádron. Nossos resultados podem ser encontrados nas Refs.[1, 2, 3, 4, $5,6]$.

Essa tese está organizada da seguinte forma:

- No capítulo 2, apresentamos algumas características da QCD. Apresentamos também o espalhamento profundamente inelástico e o modelo de pártons introduzindo as funções de distribuição de pártons e funções de estrutura. No final, mostramos como correções ao modelo de pártons devidas à QCD levam às equações DGLAP e fazemos uma breve discussão sobre a chamada análise global e a obtenção das funções de distribuição de pártons;

- No capítulo 3 abordamos diversas ferramentas importantes para o estudo da QCD em altas energias. Começamos introduzindo o formalismo de dipolo de cor, o qual é um dos ingredientes básicos de nossos trabalhos. Logo após, apresentamos as soluções das equações lineares da QCD (DGLAP e BFKL) no limite de altas energias e salientamos que as mesmas violam o vínculo de unitariedade da matriz $S$. Estudamos ainda as equações de evolução não lineares GLR (devida à Gribov, Levin e Ryskin) e BK (devida à Balitsky e Kovchegov). Aqui, introduzimos o conceito de saturação de pártons e discutimos como ele pode ser entendido através do formalismo de dipolo. A seguir, apresentamos os modelos fenomenológicos utilizados em nossos trabalhos para a seção de choque dipolo-alvo, os quais levam em conta efeitos de saturação. O capítulo se encerra com uma breve discussão a respeito de algumas evidências experimentais da física de saturação;

- No capítulo 4, estudamos o método de fótons equivalentes e mostramos como as colisões ultraperiféricas entre dois hádrons podem ser tratadas em termos de colisões fóton-hádron e fóton-fóton. Logo após, apresentamos os chamados fluxos de fótons equivalentes que são usados nos cálculos dessa tese. Em seguida, discutimos como a produção de mésons vetoriais vem sendo estudada em colisores hadrônicos. Por fim, apresentamos alguns resultados de produção de partículas em processos foto-induzidos no LHC; 
- No capítulo 5 apresentamos nossos resultados para a produção de mésons vetoriais em colisões hádron-hádron para energias disponíveis no LHC. Inicialmente, discutimos a fotoprodução difrativa de mésons vetoriais e como o formalismo de dipolo se aplica a esse tipo de processo. Logo após, apresentamos nossos resultados para a produção de $J / \psi, \Upsilon, \rho$ e $\phi$ em colisões $p p, p P b$ e $P b P b$ ultraperiféricas. Esses resultados foram publicados nas Refs. [1, 2, 6];

- No capítulo 6, estudamos a produção dupla de mésons vetoriais em interações $\gamma-\gamma$ em colisões periféricas. Primeiramente, começamos nosso estudo com uma análise detalhada do comportamento das seções de choque $\gamma \gamma \rightarrow V V$ ( $V$ denota um méson vetorial) com a energia de centro de massa $\gamma \gamma$ e com a virtualidade. Argumentamos que tal processo, poderá ser estudado em um colisor $e^{+} e^{-}$. Logo após, discutimos como esse processo pode ser estudado no LHC, através de colisões ultraperiféricas hádron-hádron e apresentamos nossos resultados. Esses resultados foram publicados nas Refs.[3, 4];

- No capítulo 7 estudamos a produção dupla de mésons vetoriais devido ao espalhamento duplo $\gamma$-hádron (mecanismo de duplo espalhamento - "double scattering mechanism" / DSM). As seções de choque do DSM são escritas em termos das seções de choque para a produção de um único méson (como discutido no capítulo 5). Aqui também fazemos uma comparação com as seções de choque obtidas no capítulo 6 para a produção dupla de mésons vetoriais em interações $\gamma \gamma$. Esses resultados foram publicados na Ref.[5];

- Por fim, apresentamos nossas conclusões e perspectivas. 


\section{Capítulo 2}

\section{Cromodinâmica Quântica}

A Cromodinâmica Quântica, é a teoria que trata da mais intensa das interações fundamentais conhecidas, a interação forte. Ela estuda a interação entre quarks e glúons. Quarks são férmions de spin-1/2, carga elétrica fracionária e portam um tipo de carga chamada carga de cor (existem três tipos de cargas de cor: azul, vermelho e verde). Os glúons são bósons de spin-1, sem massa e sem carga elétrica e, assim como os quarks, também portam carga de cor. Consequentemente, além dos glúons interagirem com quarks, podem também interagir entre si. Essa é uma característica da QCD que não está presente na Eletrodinâmica Quântica (Quantum Electrodynamics-QED).

Existem ainda duas características básicas das interações fortes que devem ser levadas em conta na construção da teoria: o confinamento e a liberdade assintótica. Em grandes distâncias, a interação forte é bastante intensa. Nesse caso a constante de acoplamento das interações fortes $\alpha_{s}$ é grande, impossibilitando o uso de métodos perturbativos. Portanto, quarks e glúons são confinados formando os hádrons (prótons, nêutrons, píons e etc.). Por outro lado, em pequenas distâncias, a intensidade da interação forte é pequena. Isso leva à liberdade assintótica, ou seja, os quarks são praticamente livres (interagem pouco entre si). Nessas condições $\alpha_{s}$ é pequeno e métodos da QCD perturbativa podem ser usados.

As duas propriedades citadas acima podem ser descritas por uma teoria de gauge não abeliana (ou seja, uma teoria cujos geradores não comutam). Para o caso da QCD, temos o grupo $S U\left(N_{c}=3\right.$ ), onde S (special) significa que o determinante do operador $U$ responsável pela transformação de gauge é igual a 1 , U (unitary) significa que o operador $U$ é unitário $\left(U^{\dagger} U=1\right)$ e $N_{c}$ é o número de cores. De fato, Gross, Wilczek e Politzer mostraram (Refs. $[7,8]$ ) que uma teoria de gauge não abeliana possui liberdade assintótica.

Nesse capítulo vamos introduzir a lagrangiana da QCD e discutir alguns conceitos básicos. Vamos também discutir a liberdade assintótica presente na QCD, mostrando como se dá a variação de $\alpha_{s}$ com a escala de momento. Para isso, usaremos os métodos de grupo de renormalização. Logo após, apresentamos os pincipais resultados do espalhamento profundamente inelástico via modelo de pártons, onde introduzimos variáveis e observáveis físicos de interesse no estudo da física de altas energias. Por fim mostramos como surgem as correções ao modelo de pártons vindas das interações da QCD. 


\subsection{A Lagragiana da QCD e a Invariância de Gauge}

A lagrangiana da QCD pode ser escrita como $[9,10]$

$$
\mathcal{L}=-\frac{1}{4} F^{a \mu \nu} F_{\mu \nu}^{a}+\bar{\psi}(\not D-m) \psi
$$

sendo $\psi$ definido por

$$
\psi=\left(\begin{array}{c}
\psi_{r} \\
\psi_{b} \\
\psi_{g}
\end{array}\right)
$$

onde os índices $r, b$ e $g$ denotam as 3 cores e $\psi_{i}(i=r, b$ e $g)$ são os campos dos quarks. Temos ainda que $\not D=\gamma^{\mu} D_{\mu}, D_{\mu}=\partial_{\mu}-i g A_{\mu}, A_{\mu} \equiv A_{\mu}^{a} t^{a}\left(A_{\mu}\right.$ são os campos dos glúons e $a=1,2, \ldots, 8)$ e $t^{a}$ são os geradores do grupo $\mathrm{SU}(3)$. Aqui $g$ é o análogo da carga elétrica elementar $e$ presente na lagrangiana da QED. Além disso, $F_{\mu \nu}=F_{\mu \nu}^{a} t^{a}$ é definido por $-i g F_{\mu \nu} \psi=\left[D_{\mu}, D_{\nu}\right] \psi$. A lagrangiana acima é invariante sob as seguintes transformações de gauge

$$
\begin{gathered}
\psi(x) \rightarrow \psi^{\prime}(x)=U(x) \psi(x) \\
\bar{\psi}(x) \rightarrow \bar{\psi}^{\prime}(x)=\bar{\psi}(x) U^{-1}(x) \\
A_{\mu}(x) \rightarrow A_{\mu}^{\prime}(x)=U(x) A_{\mu} U^{-1}(x)-\frac{i}{g}\left(\partial_{\mu} U(x)\right) U^{-1}(x),
\end{gathered}
$$

onde o operador $U(x)=e^{i t^{a} \theta^{a}(x)}$ e $\theta^{a}(x)$ é um conjunto de funções reais dependentes da posição e do tempo. De fato, com as tranformações acima, podemos mostrar que

$$
\mathcal{L}\left(\psi^{\prime}, \bar{\psi}^{\prime}, A_{\mu}^{\prime}\right)=\mathcal{L}\left(\psi, \bar{\psi}, A_{\mu}\right)
$$

ou seja, a lagrangiana é invariante sob tais transformações.

Os geradores do grupo $S U(3)$ são [10]

$$
\begin{gathered}
t^{1}=\frac{1}{2}\left(\begin{array}{lll}
0 & 1 & 0 \\
1 & 0 & 0 \\
0 & 0 & 0
\end{array}\right), \quad t^{2}=\frac{1}{2}\left(\begin{array}{ccc}
0 & -i & 0 \\
i & 0 & 0 \\
0 & 0 & 0
\end{array}\right), \quad t^{3}=\frac{1}{2}\left(\begin{array}{ccc}
1 & 0 & 0 \\
0 & -1 & 0 \\
0 & 0 & 0
\end{array}\right), \\
t^{4}=\frac{1}{2}\left(\begin{array}{lll}
0 & 0 & 1 \\
0 & 0 & 0 \\
1 & 0 & 0
\end{array}\right), \quad t^{5}=\frac{1}{2}\left(\begin{array}{ccc}
0 & 0 & -i \\
0 & 0 & 0 \\
i & 0 & 0
\end{array}\right), \quad t^{6}=\frac{1}{2}\left(\begin{array}{ccc}
0 & 0 & 0 \\
0 & 0 & 1 \\
0 & 1 & 0
\end{array}\right), \\
t^{7}=\frac{1}{2}\left(\begin{array}{ccc}
0 & 0 & 0 \\
0 & 0 & -i \\
0 & i & 0
\end{array}\right), \quad t^{8}=\frac{1}{2 \sqrt{3}}\left(\begin{array}{ccc}
1 & 0 & 0 \\
0 & 1 & 0 \\
0 & 0 & -2
\end{array}\right)
\end{gathered}
$$


os quais satisfazem as seguintes relações

$$
\operatorname{Tr}\left[t^{a}, t^{b}\right]=\frac{1}{2} \delta^{a b}
$$

e

$$
t^{a} t^{a}=\frac{N_{c}^{2}-1}{2 N_{c}}
$$

Da definição de $F_{\mu \nu}$, temos

$$
F_{\mu \nu}=F_{\mu \nu}^{a} t^{a}=\partial_{\mu} A_{\nu}-\partial_{\nu} A_{\mu}-i g\left[A_{\mu}, A_{\nu}\right]
$$

Para o caso de uma teoria de gauge abeliana, o comutador acima se anula (como ocorre na QED, por exemplo). Entretanto, para uma teoria de gauge não-abeliana, temos $\left[A_{\mu}, A_{\nu}\right]=$ $A_{\mu}^{a} A_{\nu}^{b}\left[t^{a}, t^{b}\right]$ com $\left[t^{a}, t^{b}\right]=i f^{a b c} t^{c}$, sendo $f^{a b c}$ as constantes de estrutura do grupo $S U(3)$ (as quais são totalmente antissimétricas). Isto nos leva à seguinte forma para $F_{\mu \nu}^{a}$

$$
F_{\mu \nu}^{a}=\partial_{\mu} A_{\nu}^{a}-\partial_{\nu} A_{\mu}^{a}+g f^{a b c} A_{\mu}^{b} A_{\nu}^{c} .
$$

A lagrangiana acima (Eq.(2.1)) é chamada de lagrangiana clássica da QCD. Das Eqs.(2.1) e (2.10), podemos observar uma característica marcante da QCD, que não está presente na QED. Devido ao termo novo que surge em $F^{a \mu \nu}$, surgem novos tipos de interações (vértices). Além da interação férmion-férmion-bóson $(F F B)$, já presente na QED, podemos observar que esse novo termo de $F^{a \mu \nu}$ permite que glúons possam interagir entre si através dos vértices $B B B$ e $B B B B$.

Entretanto, a Eq.(2.1) não é a forma completa da lagrangiana da QCD, devido à uma impossibilidade de determinação do propagador do glúon sem a fixação de um gauge. Isso ocorre por causa da liberdade de escolha de gauge na Eq.(2.5), tornando o campo $A_{\mu}$ um campo arbitrário. Devemos incluir um termo de fixação de gauge $\left(\mathcal{L}_{\text {fix }}\right)$ que em sua forma covariante será $[9,10]$

$$
\mathcal{L}_{\text {fix }}=-\frac{1}{2 \xi}\left(\partial^{\mu} A_{\mu}^{a}\right)^{2}
$$

Mas um termo de fixação de gauge covariante, como o definido acima dá origem a graus de liberdade não físicos. Isso requer que este termo de fixação de gauge venha acompanhado dos campos fantasmas de Fadeev-Popov, que são incluídos na lagrangiana através do termo $\left(\mathcal{L}_{\text {ghost }}\right)$

$$
\mathcal{L}_{\text {ghost }}=\left(\partial_{\mu} c^{a *}\right)\left(\delta^{a c} \partial^{\mu}+g f^{a b c} A_{\mu}^{b}\right) c^{c},
$$

sendo $c^{a}(x)$ os campos fantasmas, que são escalares de Lorentz, e são responsáveis por cancelar os graus de liberdade não físicos. Uma escolha de gauge bastante comum é o gauge de Lorenz, onde

$$
\partial^{\mu} A_{\mu}^{a}=0
$$




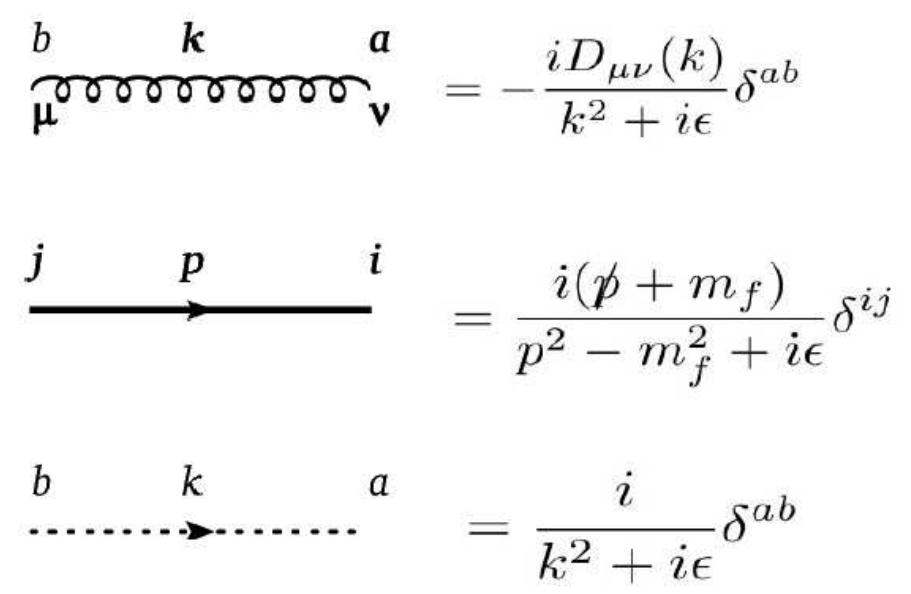

Fig. 2.1: Propagadores da QCD. A figura foi retirada da Ref.[9].

Outra escolha de gauge possível é o gauge do cone de luz, definido por

$$
\eta \cdot A^{a}=\eta^{\mu} A_{\mu}^{a}=0
$$

onde $\eta^{\mu}$ é um quadrivetor constante do tipo luz (isto é, $\eta^{2}=0$ ). Nessa escolha particular de gauge, os campos fantasmas não se acoplam aos campos de glúons. Usando o formalismo de integrais de trajetória é possível mostrar que não existem campos fantasmas no gauge de cone do luz [10]. Nesse caso

$$
\mathcal{L}_{f i x}=-\frac{1}{2 \xi}\left(\eta^{\mu} A_{\mu}^{a}\right)^{2}, \xi \rightarrow 0
$$

Nas Figs.2.1 e 2.2 apresentamos as regras de Feynman da QCD. A Fig.2.1 mostra os propagadores de glúons (linha em forma de mola), quarks (linha contínua) e fantasmas (linha tracejada). Os vértices de interação são mostrados na Fig.2.2.

O fator $D_{\mu \nu}(k)$ no propagador do glúon (Fig.2.1) é dado por

$$
D_{\mu \nu}(k)=g_{\mu \nu}-(1-\xi) \frac{k_{\mu} k_{\nu}}{k^{2}}
$$

no gauge de Lorenz, onde usualmente escolhemos $\xi=0$ (gauge de Landau) ou $\xi=1$ (gauge de Feynman). No gauge do cone de luz temos

$$
D_{\mu \nu}(k)=g_{\mu \nu}-\frac{\eta_{\mu} k_{\nu}+\eta_{\nu} k_{\mu}}{\eta \cdot k}
$$



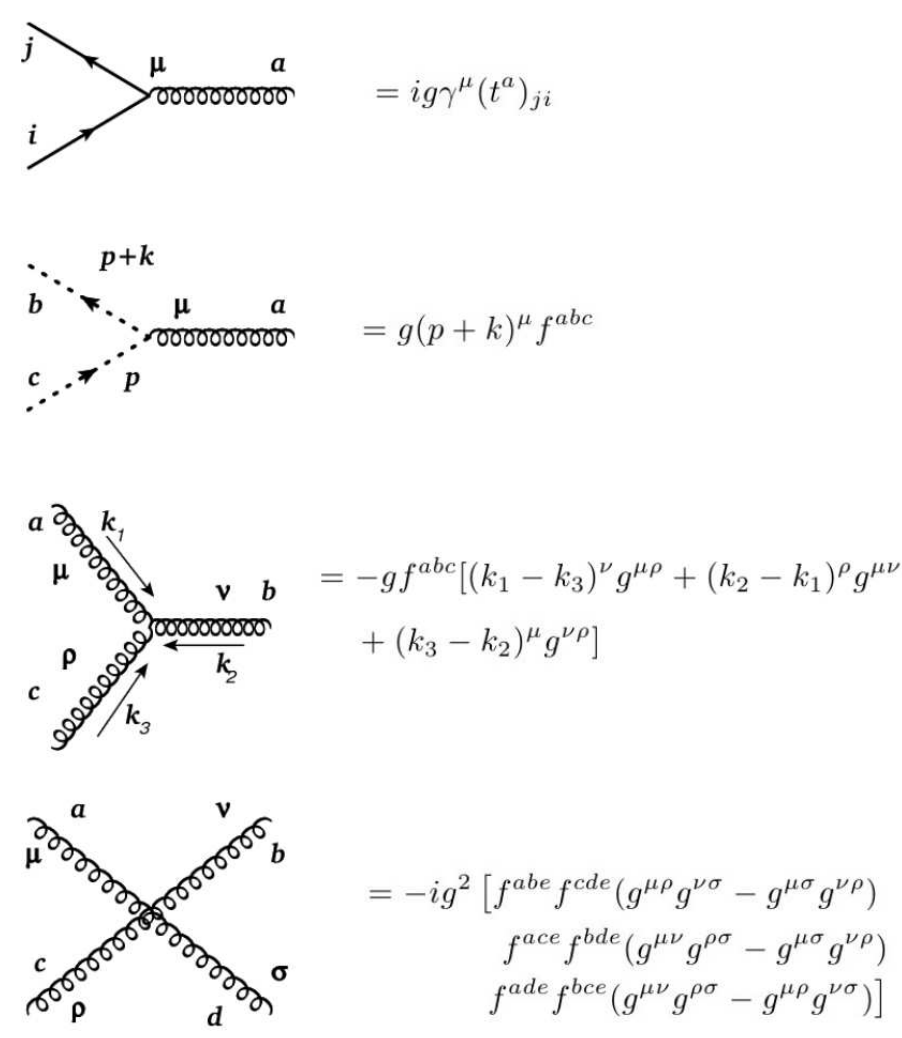

Fig. 2.2: Vértices da QCD. A figura foi retirada da Ref.[9].

\subsection{A Constante de Acoplamento da QCD e a Liberdade Assintótica}

Em teoria quântica de campos, a expansão perturbativa da amplitude de espalhamento além da ordem dominante é em geral divergente (ver Ref.[10]). A forma de regular as eventuais divergências é através dos chamados métodos de renormalização. Uma teoria de campos renormalizável é aquela em que a renormalização de um número finito de parâmetros assegura resultados finitos para cálculos em todas as ordens em teoria de perturbação. Isso faz com que parâmetros considerados como constantes inicialmente, possam depender da escala do processo em estudo. Mais especificamente, os parâmetros da lagrangiana como 
massa, constante de acoplamento e os campos, devem ser redefinidos (os parâmetro devem ser renormalizados) de forma a absorverem as divergências.

O processo de renormalização requer que os parâmetros renormalizados sejam definidos em uma dada escala $\mu^{2}$, a qual é arbitrária (em geral, a medida experimental de um observável em uma dada escala $\mu$ ). Mas um certo observável físico não pode depender de uma escolha de escala. Por exemplo, um parâmetro renormalizado de uma teoria (a constante de acoplamento, por exemplo) pode ser definido em várias escalas diferentes. Mas um observável físico (uma seção de choque, por exemplo), calculado perturbativamente, não pode depender de uma particular escolha de escala onde os parâmetros foram definidos. Dizemos que tal observável deve ser invariante frente ao grupo de todas as possíveis escolhas de escala. Esse fato é explorado pelo chamado método do grupo de renormalização [9, 10, 11], que será brevemente discutido a seguir.

Vamos considerar um observável adimensional $\Gamma$ (uma razão entre seções de choque, por exemplo) envolvendo apenas uma escala $Q^{2}$. Tal observável pode ser escrito como (ver Ref. [11]) $\Gamma=\Gamma\left(Q^{2} / \mu^{2}, \alpha_{s}\left(\mu^{2}\right)\right)$, onde $\alpha_{s}\left(\mu^{2}\right)\left(=g^{2} /(4 \pi)\right)$ é a constante de acoplamento renormalizada da QCD definida na escala $\mu^{2}$. O fato de $\Gamma$ não depender da escala é expresso por

$$
\mu^{2} \frac{\mathrm{d} \Gamma\left(Q^{2} / \mu^{2}, \alpha_{s}\left(\mu^{2}\right)\right)}{\mathrm{d} \mu^{2}}=0
$$

o que leva à equação do grupo de renormalização

$$
\left\{\mu^{2} \frac{\partial}{\partial \mu^{2}}+\beta\left(\alpha_{s}\right) \frac{\partial}{\partial \alpha_{s}}\right\} \Gamma\left(Q^{2} / \mu^{2}, \alpha_{s}\left(\mu^{2}\right)\right)=0
$$

onde definimos a função beta da QCD (a qual pode ser calculada perturbativamente)

$$
\beta\left(\alpha_{s}\right)=\mu^{2} \frac{\partial \alpha_{s}\left(\mu^{2}\right)}{\partial \mu^{2}}
$$

Temos que uma mudança de escala $\mu^{2} \rightarrow \mu_{2}^{2}$, deve ser compensada por uma mudança na constante de acoplamento $\alpha_{s}\left(\mu^{2}\right) \rightarrow \alpha_{s}\left(\mu_{2}^{2}\right)$, de forma que o observável seja independente dessa mudança

$$
\Gamma\left(Q^{2} / \mu^{2}, \alpha_{s}\left(\mu^{2}\right)\right)=\Gamma\left(Q^{2} / \mu_{2}^{2}, \alpha_{s}\left(\mu_{2}^{2}\right)\right)
$$

Definindo a variável $t=\ln \left(\mu_{2}^{2} / \mu^{2}\right)$, temos

$$
\beta\left(\alpha_{s}\left(\mu_{2}^{2}\right)\right)=\frac{\partial \alpha_{s}\left(\mu_{2}^{2}\right)}{\partial t} .
$$

A equação acima pode ser escrita como

$$
t=\ln \frac{\mu_{2}^{2}}{\mu^{2}}=\int_{\alpha_{s}\left(\mu^{2}\right)}^{\alpha_{s}\left(\mu_{2}^{2}\right)} \frac{\mathrm{d} x}{\beta(x)} .
$$

A função beta da QCD é conhecida e seu termo em mais baixa ordem em $\alpha_{s}$ é [8, 11]

$$
\beta\left(\alpha_{s}=x\right)=-b_{0} x^{2}, \quad b_{0}=\frac{33-2 n_{f}}{12 \pi},
$$


onde $n_{f}$ é o número de sabores ativos. Com isso, a Eq.(2.23) fornece

$$
\alpha_{s}\left(\mu_{2}^{2}\right)=\frac{\alpha_{s}\left(\mu^{2}\right)}{1+b_{0} \alpha_{s}\left(\mu^{2}\right) \ln \left(\mu_{2}^{2} / \mu^{2}\right)} .
$$

É comum definir a escala de confinamento da QCD $\Lambda_{Q C D}$ como sendo dada por (a qual é determinada experimentalmente) [11]

$$
\ln \Lambda_{Q C D}^{2}=\ln \mu^{2}-\frac{1}{b_{0} \alpha_{s}\left(\mu^{2}\right)} .
$$

Então, tomando $\mu_{2}^{2}=Q^{2}$, a Eq.(2.25) fornece

$$
\alpha_{s}\left(Q^{2}\right)=\frac{1}{b_{0} \ln \left(Q^{2} / \Lambda_{Q C D}^{2}\right)} .
$$

A equação acima nos diz que a constante de acoplamento diminui com o aumento da escala de momento (diminuição da distância), sendo que no caso $Q^{2} \gg \Lambda_{Q C D}^{2}\left(\operatorname{com} \Lambda_{Q C D} \approx\right.$ $200-300 \mathrm{MeV}$ ), temos uma constante de acoplamento pequena, possibilitando cálculos perturbativos. Além disso, nessas condições, os quarks são aproximadamente livres. Esse fenômeno é chamado de liberdade assintótica. Por outro lado, se $Q^{2} \rightarrow \Lambda_{Q C D}^{2}$, as interações entre quarks e glúons se tornam muito intensas, e métodos perturbativos não são adequados para tratar o problema.

\subsection{O Espalhamento Profundamente Inelástico}

O espalhamento profundamente inelástico (Deep Inelastic Scattering - DIS) é uma importante ferramenta para o estudo da estrutura interna dos hádrons, bem como pode ser usado como um teste da QCD perturbativa. No DIS, um elétron (que é uma partícula sem estrutura interna) colide com um hádron (um próton, por exemplo) para que possamos obter informações sobre a estrutura interna deste último.

Inicialmente, o elétron age como uma fonte de fótons virtuais (denotados por $\gamma^{*}$ ), que colidem com o próton. Para uma colisão com momento transferido suficientemente grande o fóton virtual consegue resolver objetos puntuais (com tamanho da ordem do inverso do momento transferido) dentro do próton. Tais objetos puntuais (sem estrutura interna) são chamados de pártons. Além disso, para grandes momenta transferidos, o espalhamento é inelástico, provocando a quebra do próton.

No chamado modelo de pártons (para uma revisão, veja a Ref.[11]), que será brevemente apresentado, considera-se que a seção de choque do DIS entre o elétron e o próton é dada pela soma incoerente das seções de choque elásticas (devido à interação eletromagnética) entre o elétron e cada párton individual. Nesse modelo considera-se que os pártons não interagem entre si. Essa forma de tratar o DIS leva às definições das funções de distribuição de pártons e das funções de estrutura e serão discutidas mais adiante.

As funções de estrutura são observáveis físicos que apresentam o chamado scaling de Bjorken [12], isto é, elas são aproximadamente independentes do momento transferido. Experimentalmente (ver Ref.[13, 14]) esse resultado é válido apenas para energias moderadas. 


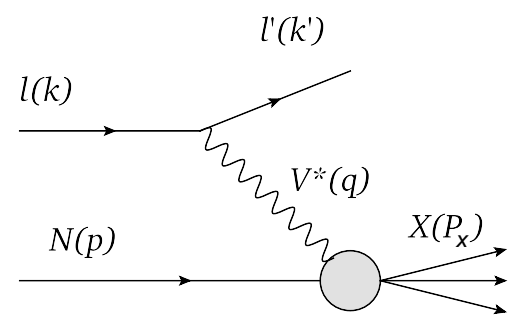

Fig. 2.3: Espalhamento profundamente inelástico elétron - próton.

A violação do scaling de Bjorken é explicada pela QCD. Pártons são identificados como quarks e glúons e ao levarmos em conta a interação entre eles, obtemos correções que levam à violação do scaling de Bjorken. Essas correções levam às chamadas equações de evolução DGLAP (devidas à Dokshitzer, Gribov, Lipatov, Altarelli e Parisi [15, 16, 17]) para as funções de distribuição de quarks e glúons. Essas equações descrevem a evolução das funções de distribuições de quarks e glúons com o momento transferido.

Nessa seção vamos estudar as seções de choque do DIS e definir as funções de distribuição de pártons, bem como as funções de estrutura. Por fim, vamos mostrar como a violação do scaling de Bjorken leva às equações de evolução DGLAP apresentando alguns de seus aspectos.

\subsubsection{Cinemática do DIS}

Vamos apresentar a cinemática para o processo lépton-hádron $l N \rightarrow l^{\prime} X$. Associamos os 4 -vetores $k$ e $k^{\prime}$ para os léptons incidente e emergente, respectivamente, e $p$ para o alvo. O processo é mediado por um bóson vetorial ( $\gamma, W$ ou $Z$ ) com quadrimomento (ver Fig.2.3)

$$
q=k-k^{\prime},
$$

sendo o quadrimomento do estado final $X$ dado por

$$
P_{X}=p+q
$$

Os seguintes invariantes de Lorentz são definidos para a descrição cinemática do processo:

$$
s=(p+k)^{2},
$$

que é a energia do CM ao quadrado,

$$
W^{2}=(q+p)^{2} \quad\left(\equiv P_{X}^{2}\right),
$$


que é a energia de CM ao quadrado do sistema $\gamma^{*} p$ (ou ainda a massa invariante ao quadrado do sistema $X$ ),

$$
Q^{2}=-q^{2}
$$

que é o negativo do quadrimomento transferido ao quadrado (chamado de virtualidade do fóton),

$$
\nu=\frac{p \cdot q}{m_{p}}=E-E^{\prime}
$$

que é a diferença entre as energias inicial e final do elétron no referencial de laboratório,

$$
x=\frac{Q^{2}}{2 p \cdot q}=\frac{Q^{2}}{2 m_{p} \nu}=\frac{Q^{2}}{Q^{2}+W^{2}-m_{p}^{2}},
$$

que é a variável de Bjorken, a qual é interpretada como a fração de momento do núcleon incidente carregada pelo parton interagente e

$$
y=\frac{p \cdot q}{p \cdot k}=\frac{W^{2}+Q^{2}-m_{p}^{2}}{s-m_{p}^{2}},
$$

que é a medida da quantidade de energia transferida entre o lépton e o sistema hadrônico.

Podemos mostrar que

$$
\begin{aligned}
s x y & =\left[(p+k)^{2}\right]\left[\frac{Q^{2}}{2 p \cdot q}\right]\left[\frac{p \cdot q}{p \cdot k}\right] \\
& =\left[m_{p}^{2}+m_{e}^{2}+2 p \cdot k\right]\left[\frac{Q^{2}}{2 p \cdot q}\right]\left[\frac{p \cdot q}{p \cdot k}\right]
\end{aligned}
$$

que leva a (usando $p^{2} \approx k^{2} \approx 0$, válido para pequenas massas)

$$
Q^{2}=s x y \text {. }
$$

A equação acima nos mostra que o limite de altas energias corresponde ao limite de pequeno $x$.

No modelo de pártons, temos que o núcleon é composto por centros espalhadores puntuais e não interagentes (pártons). A seção de choque lépton-hádron é aproximada por uma soma incoerente de espalhamentos lépton-párton via troca de um bóson vetorial. Um exemplo pode ser visto na Fig.2.4.

\subsubsection{Espalhamento elástico elétron-quark}

Nessa subseção, apresentamos alguns resultados do modelo a pártons para o DIS, a fim de interpretar o espalhamento lépton-hádron em termos do espalhamento lépton-párton. Para um próton, as possíveis cargas dos quarks serão $\frac{2}{3} e$ ou $-\frac{1}{3} e$, o que nos leva a

$$
e^{\prime}=e_{i} e
$$




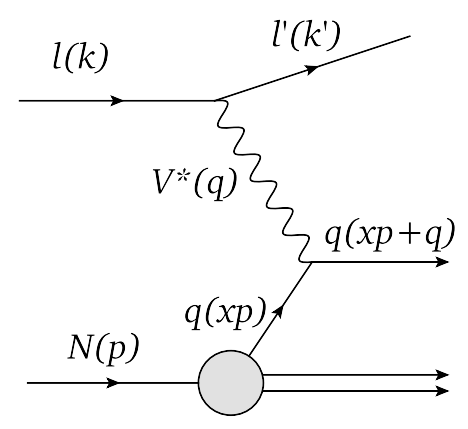

Fig. 2.4: Espalhamento elétron - párton.

sendo $e_{i}$ a fração que multiplica $e$. Além disso, as variáveis cinemáticas $s, Q^{2}$ e $y$ devem estar adequadas para o espalhamento elétron-quark, lembrando que o quark interagente carrega uma fração $x$ do momento do hádron pai. A virtualidade $Q^{2}$ (momento transferido) e a energia que o lépton transfere ao hádron $y$ permanecem inalterados. Por outro lado como $p \rightarrow x p$, então $s \rightarrow x s$, sendo $s\left(\approx 2 k \cdot p_{\text {quark }}\right)$ a energia de CM lépton-quark. A seção de choque para o espalhamento elétron-quark para um dado valor de $x$ será [11]

$$
\frac{\mathrm{d} \hat{\sigma}}{\mathrm{d} y}=\frac{2 \pi \alpha^{2}}{Q^{4}}\left[1+(1-y)^{2}\right] x s e_{i}^{2} .
$$

onde $\hat{\sigma}$ denota a seção de choque do processo elétron-párton.

Para obtermos o espalhamento elétron-hádron da Eq.(2.38), vamos definir algumas quantidades. Uma delas é $f_{i}(x)$, que é a função de distribuição que dá a probabilidade de que o quark interagente $i$ carregue uma fração $x$ do momento $p$ do hádron. Essa quantidade fornece a distribuição de pártons (densidade de pártons). Outra quantidade é $x f_{i}(x)$ que é a distribuição de momento (ou densidade de momento), também chamada de função de distribuição partônica (Parton Distribution Function - PDF).

Com essas definições a seção de choque duplamente diferencial para o espalhamento incoerente devido a todos os tipos possíveis de quarks com um dado valor de $x$ é dada pela soma incoerente das seções de choque dos componentes individuais do alvo [18].

$$
\begin{aligned}
\frac{\mathrm{d}^{2} \sigma}{\mathrm{d} x \mathrm{~d} y} & =\sum_{i} f_{i}(x) \frac{\mathrm{d} \hat{\sigma}}{\mathrm{d} y} \\
& =\frac{2 \pi \alpha^{2}}{Q^{2}}\left[1+(1-y)^{2}\right] s \sum_{i} e_{i}^{2} x f_{i}(x)
\end{aligned}
$$

ou, de forma equivalente

$$
\frac{\mathrm{d}^{2} \sigma}{\mathrm{d} x \mathrm{~d} Q^{2}}=\frac{2 \pi \alpha^{2}}{x Q^{4}}\left[1+(1-y)^{2}\right] \sum_{i} e_{i}^{2} x f_{i}(x) .
$$


A seguir calculamos o espalhamento elétron-hádron, cuja seção de choque será comparada com a dada pela equação acima. Como veremos, isso nos fornecerá informações e resultados importantes com respeito ao DIS.

\subsubsection{Espalhamento inelástico elétron - hádron}

Vamos desenvolver o modelo de pártons para o espalhamento lépton-hádron. Analogamente ao tensor leptônico, vamos introduzir o tensor hadrônico ( $W^{\mu \nu}$, definido nas Refs. [9, $11,18]$ ), tal que a seção de choque no referencial de laboratório (referencial de repouso do próton) é $[9,11]$

$$
\frac{\mathrm{d}^{2} \sigma}{\mathrm{d} \Omega \mathrm{d} E^{\prime}}=\frac{\alpha^{2}}{q^{4}} \frac{E^{\prime}}{E} L \cdot W
$$

onde $E$ e $E^{\prime}$ são, respectivamente, as energias do elétron incidente e espalhado e d $\Omega$ é o ângulo sólido num ângulo de espalhamento $\theta$. O tensor hadrônico é dado por $[9,11,18]$

$$
\begin{aligned}
W^{\mu \nu} & =-W_{1}\left(x, Q^{2}\right)\left(g^{\mu \nu}-\frac{q^{\mu} q^{\nu}}{q^{2}}\right) \\
& +\frac{W_{2}\left(x, Q^{2}\right)}{m^{2}}\left(p^{\mu}-\frac{p \cdot q}{q^{2}} q^{\mu}\right)\left(p^{\nu}-\frac{p \cdot q}{q^{2}} q^{\nu}\right)
\end{aligned}
$$

onde $W_{i}$ são chamadas de constantes de estrutura. Com a equação acima, é possível mostrar que $[11]$

$$
L \cdot W=4\left(k^{\prime} \cdot k\right) W_{1}+\frac{2 W_{2}}{m^{2}}\left[2(p \cdot k)\left(p \cdot k^{\prime}\right)-m^{2}\left(k \cdot k^{\prime}\right)\right] .
$$

No referencial de repouso do próton, temos

$$
\begin{aligned}
k \cdot p & =m E \\
k^{\prime} \cdot p & =m E^{\prime}
\end{aligned}
$$

e

$$
\begin{aligned}
Q^{2} & =-\left(k-k^{\prime}\right)^{2} \\
& =4 E E^{\prime} \operatorname{sen}^{2} \frac{\theta}{2}
\end{aligned}
$$

o que nos permite escrever

$$
\frac{\mathrm{d}^{2} \sigma}{\mathrm{d} \Omega \mathrm{d} E^{\prime}}=\frac{\alpha^{2}}{4 E^{2} \operatorname{sen}^{4} \frac{\theta}{2}}\left(W_{2} \cos ^{2} \frac{\theta}{2}+2 W_{1} \operatorname{sen}^{2} \frac{\theta}{2}\right) .
$$


Vamos agora escrever a equação acima de outra forma. Das relações entre as variáveis cinemáticas para o referencial de laboratório, é possível mostrar que

$$
x=\frac{2 E E^{\prime} \operatorname{sen}^{2} \frac{\theta}{2}}{m\left(E-E^{\prime}\right)}
$$

e

$$
y=1-\frac{E^{\prime}}{E}
$$

o que leva a

$$
\frac{\mathrm{d}^{2} \sigma}{\mathrm{d} x \mathrm{~d} y}=\frac{4 \pi \alpha^{2} s}{Q^{4}}\left[x y^{2} F_{1}(x, y)+(1-y) F_{2}(x, y)\right],
$$

onde definimos as funções de estrutura $F_{1}=m W_{1}$ e $F_{2}=\nu W_{2}$. Definindo $F_{L}=F_{2}-2 x F_{1}$ e $Y_{+}=1+(1-y)^{2}$, temos

$$
\frac{\mathrm{d}^{2} \sigma}{\mathrm{d} x \mathrm{~d} Q^{2}}=\frac{2 \pi \alpha^{2}}{x Q^{4}}\left[Y_{+} F_{2}\left(x, Q^{2}\right)-y^{2} F_{L}\left(x, Q^{2}\right)\right]
$$

Comparando a equação acima com a Eq.(2.40), obtemos alguns resultados importantes do modelo de pártons. Primeiro,

$$
F_{2}\left(x, Q^{2}\right)=\sum_{i} e_{i}^{2} x f_{i}(x)
$$

e além disso,

$$
F_{L}\left(x, Q^{2}\right)=0
$$

que nos leva à relação de Callan-Gross $F_{2}\left(x, Q^{2}\right)=2 x F_{1}\left(x, Q^{2}\right)$ [19]. Além disso, vemos que as funções de estrutura não dependem de $Q^{2}$ e dependem apenas de $x$. Esse resultado é o scaling de Bjorken citado anteriormente.

Por fim, a seção de choque para o processo elétron-hádron pode ser relacionada com a seção de choque para o processo fóton virtual-próton. Podemos mostrar [11] que as seções de choque $\gamma^{*} p$ transversa $\left(\sigma_{T}\right)$ e longitudinal $\left(\sigma_{L}\right)$ se relacionam com as funções de estrutura do DIS por

$$
\sigma_{T}=\frac{4 \pi^{2} \alpha}{Q^{2}} 2 x F_{1}
$$

e

$$
\sigma_{T}+\sigma_{L}=\frac{4 \pi^{2} \alpha}{Q^{2}} F_{2}
$$

A seção de choque do DIS se relaciona com as seções de choque do processo $\gamma^{*} p$ por

$$
\frac{\mathrm{d}^{2} \sigma}{\mathrm{d} x \mathrm{~d} Q^{2}}=\Gamma\left(\sigma_{T}+\epsilon \sigma_{L}\right)
$$


onde $\Gamma$ é o fluxo e $\epsilon$ a polarização do fóton virtual, definidos por

$$
\Gamma=\frac{\alpha\left[1+(1-y)^{2}\right]}{2 \pi x Q^{2}}
$$

$\mathrm{e}$

$$
\epsilon=\frac{2(1-y)}{1+(1-y)^{2}}
$$

Nessa seção. definimos as funções de estrutura e vimos que, o modelo de pártons prediz o scaling de Bjorken. Entretanto, para $x$ suficientemente pequeno $(x \approx 0,01)$ o scaling de Bjorken é violado e as funções de estrutura passam a depender de $Q^{2}$ [13, 14] (ver Fig.2.5). Essa violação de scaling é explicada ao levarmos em conta a interação entre os pártons. Isso leva às chamadas equações de evolução DGLAP, como será visto na próxima seção.

\subsection{A violação do scaling de Bjorken e as equações DGLAP}

O modelo de pártons é a aproximação de ordem zero do mundo real, já que os constituintes dos hádrons podem interagir entre si. Eles são descritos pela QCD, a teoria de interação entre quarks e glúons. Experimentalmente, observamos que o scaling predito pelo modelo de pártons é violado e as funções de estrutura dependem de $Q^{2}$ (ver Fig.2.5). Vamos agora ver como esse comportamento surge na QCD perturbativa.

A função de estrutura $F_{2}(x)$ pode ser escrita como

$$
F_{2}(x)=\sum_{q, \bar{q}} \int_{x}^{1} \mathrm{~d} \xi f_{q}(\xi) \hat{F}_{2}^{q}\left(\frac{x}{\xi}\right),
$$

onde $\hat{F}_{2}^{q}$ é a função de estrutura elementar de quarks. De fato, se na equação acima tomamos

$$
\hat{F}_{2}^{q}(z)=e_{q}^{2} \delta(1-z)
$$

vemos que essa se reduz a Eq.(2.48).

Se não existisse interação entre quarks e glúons, a equação acima estaria pronta. Entretanto, existem outros tipos de processos que contribuem para o espalhamento $\gamma^{*} q$ (dois primeiros diagramas da Fig.2.6). Em ordem $\alpha_{s}^{1}$ temos correções de glúons real e vitual [11] e no cálculo desses diagramas surgem singularidades. Para a emissão de glúons real, temos a singularidade colinear, que surge no canal $\hat{t}$ quando $\hat{t} \rightarrow 0$, ou seja, $\operatorname{como} \hat{t} \propto(1-\cos \theta)$ essa divergência ocorre quando o glúon emitido é colinear ao quark que o emitiu. Existe também a singularidade devido a emissão de glúons soft, que é cancelada quando as contribuições reais e virtuais são somadas [18]. Portanto, vamos trabalhar apenas com a primeira.

Podemos regularizar a singularidade colinear introduzindo um corte $k_{0}^{2}$ inferior no momento transversal $k_{\perp}^{2}$ do quark interagente. $\operatorname{Em} \mathcal{O}\left(\alpha_{s}\right)$, os dois primeiros diagramas da Fig.2.6 (emissão real de glúon) contribuem para $\hat{F}_{2}^{q}$ como

$$
\hat{F}_{2}^{q}(z)=\frac{\alpha_{s}}{2 \pi} e_{q}^{2} z\left[P(z) \ln \frac{Q^{2}}{k_{0}^{2}}+h(z)\right],
$$




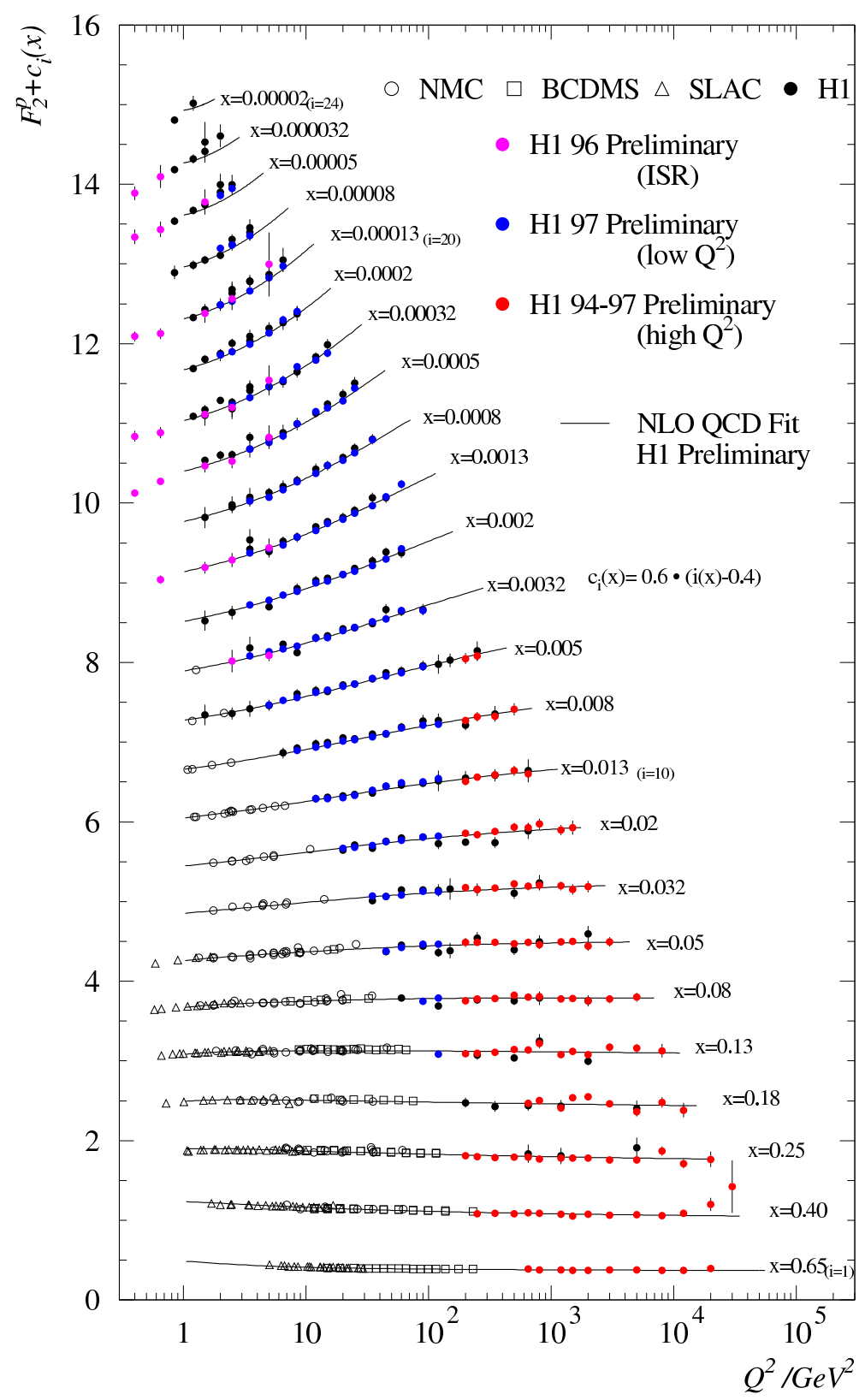

Fig. 2.5: Função de estrutura $F_{2}$ em função da virtualidade, retirada da Ref.[13].

onde $P(z)$ e $h(z)$ são funções finitas. 

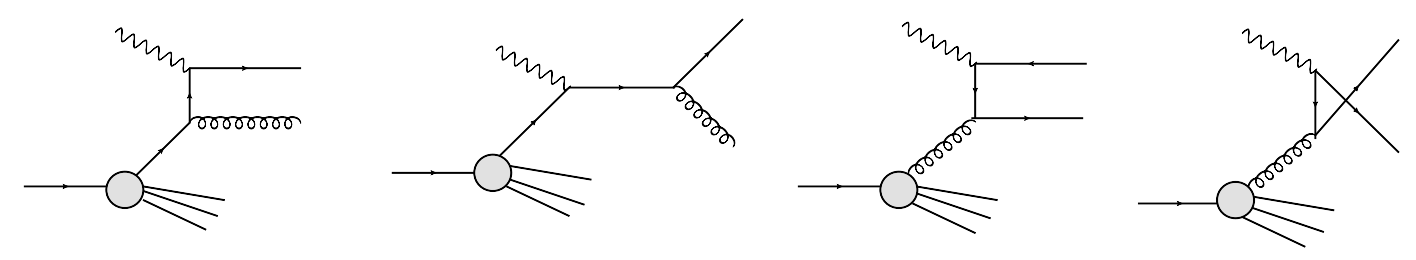

Fig. 2.6: Diagramas que contribuem em ordem $\alpha_{s}$ para o espalhamento profundamente inelástico.

Agora, podemos reescrever a Eq.(2.55) como

$$
\begin{aligned}
& F_{2}\left(x, Q^{2}\right)= \sum_{q, \bar{q}} \\
& e_{q}^{2} x\left\{f_{q}^{0}(x)+\frac{\alpha_{s}}{2 \pi} \int_{x}^{1} \frac{\mathrm{d} \xi}{\xi} f_{q}^{0}(\xi)\right. \\
&\left.\times\left[P\left(\frac{x}{\xi}\right) \ln \frac{Q^{2}}{k_{0}^{2}}+h\left(\frac{x}{\xi}\right)\right]\right\} .
\end{aligned}
$$

Iremos introduzir a escala de fatorização $\mu^{2}$ de tal forma que o logaritmo da equação acima possa ser dividido em dois na forma

$$
\ln \left(\frac{Q^{2}}{k_{0}^{2}}\right)=\ln \left(\frac{Q^{2}}{\mu^{2}}\right)+\ln \left(\frac{\mu^{2}}{k_{0}^{2}}\right)
$$

e também separamos a função $h(z)$ como

$$
h(z)=\tilde{h}(z)+h^{\prime}(z) .
$$

Vamos absorver a singularidade $\ln \mu^{2} / k_{0}^{2}$ e o termo $h^{\prime}(z)$ em uma redefinição da distribuição de quark

$$
q\left(x, \mu^{2}\right)=f_{q}^{0}+\frac{\alpha_{s}}{2 \pi} \int_{x}^{1} \frac{\mathrm{d} \xi}{\xi} f_{q}^{0}(\xi)\left[P\left(\frac{x}{\xi}\right) \ln \frac{\mu^{2}}{k_{0}^{2}}+h^{\prime}\left(\frac{x}{\xi}\right)\right]+\ldots
$$

Em termos de $F_{2}\left(x, Q^{2}\right)$, podemos escrever

$$
F_{2}\left(x, Q^{2}\right)=\sum_{q, \bar{q}} e_{q}^{2} x \int_{x}^{1} \frac{\mathrm{d} \xi}{\xi} q\left(\xi, \mu^{2}\right) C\left(\frac{x}{\xi}, Q^{2}, \mu^{2}\right)
$$

onde definimos as funções coeficiente

$$
C\left(z, Q^{2}, \mu^{2}\right)=\delta(1-z)+\frac{\alpha_{s}}{2 \pi}\left[P(z) \ln \frac{Q^{2}}{\mu^{2}}+\tilde{h}(z)\right]+\ldots
$$


Como $F_{2}\left(x, Q^{2}\right)$ é um observável físico, ela não pode depender da escala $\mu^{2}$. Então, impondo $\partial F_{2} / \partial \ln \mu^{2}=0$, temos

$$
\sum_{q, \bar{q}} e_{q}^{2} \int_{x}^{1} \frac{\mathrm{d} \xi}{\xi}\left\{\frac{\partial q\left(\xi, \mu^{2}\right)}{\partial \ln \mu^{2}} C\left(\frac{x}{\xi}, Q^{2}, \mu^{2}\right)+q\left(\xi, \mu^{2}\right) \frac{\partial}{\partial \ln \mu^{2}} C\left(\frac{x}{\xi}, Q^{2}, \mu^{2}\right)\right\}=0
$$

o que em ordem $\alpha_{s}$ dá

$$
\frac{\partial q\left(x, \mu^{2}\right)}{\partial \ln \mu^{2}}=\frac{\alpha_{s}}{2 \pi} \int_{x}^{1} \frac{\mathrm{d} \xi}{\xi} P\left(\frac{x}{\xi}\right) q\left(\xi, \mu^{2}\right)
$$

A equação acima é conhecida como equação DGLAP (Dokshitzer, Gribov, Lipatov, Altarelli e Parisi $[15,16,17])$. A função de splitting $P(x)$ representa a probabilidade de um quark emitir outro quark com fração de momento $x$ e pode ser calculada perturbativamente $[9,11]$

$$
P(x)=\sum_{n} \alpha_{s}^{n} P^{(n)}(x)
$$

$\operatorname{Em} \mathcal{O}\left(\alpha_{s}^{0}\right)$ nas funções de splitting e $\mathcal{O}\left(\alpha_{s}^{1}\right)$ nas funções coeficiente, a equação DGLAP ressoma contribuições do tipo $\left(\alpha_{s} \ln Q^{2}\right)^{n}$.

Embora tenhamos restringido nosso tratamento para quarks e antiquarks, podemos, de forma análoga, pensar na distribuição de glúons $g\left(x, Q^{2}\right)$, uma vez que podemos ter um espalhamento do tipo $\gamma^{*} g \rightarrow q \bar{q}$ (como mostram os dois últimos diagramas da Fig.2.6). Além disso, definindo a distribuição não-singleto de quarks

$$
q_{N S}\left(x, Q^{2}\right)=q\left(x, Q^{2}\right)-\bar{q}\left(x, Q^{2}\right)
$$

e a distribuição singleto

$$
\Sigma\left(x, Q^{2}\right)=\sum_{i}\left[q_{i}\left(x, Q^{2}\right)+\bar{q}_{i}\left(x, Q^{2}\right)\right]
$$

podemos mostrar que as equações DGLAP podem ser escritas como (tomando $t=\ln Q^{2} / \mu^{2}$ )

$$
\frac{\partial q_{N S}(x, t)}{\partial t}=\frac{\alpha_{s}}{2 \pi} \int_{x}^{1} \frac{\mathrm{d} \xi}{\xi} P\left(\frac{x}{\xi}\right) q_{N S}(\xi, t)
$$

e

$$
\frac{\partial}{\partial t}\left(\begin{array}{c}
\Sigma(x, t) \\
g(x, t)
\end{array}\right)=\frac{\alpha_{s}(t)}{2 \pi} \int_{x}^{1} \frac{\mathrm{d} \xi}{\xi}\left(\begin{array}{cc}
P_{q q}\left(\frac{x}{\xi}\right) & 2 n_{f} P_{q g}\left(\frac{x}{\xi}\right) \\
P_{g q}\left(\frac{x}{\xi}\right) & P_{g g}\left(\frac{x}{\xi}\right)
\end{array}\right)\left(\begin{array}{c}
\Sigma(\xi, t) \\
g(\xi, t)
\end{array}\right)
$$

onde $n_{f}$ é o número de sabores ativos.

As funções de splitting em LO são dadas por [11, 18]

$$
\begin{gathered}
P_{q q}(z)=\frac{4}{3}\left[\frac{1+z^{2}}{(1-z)_{+}}+\frac{3}{2} \delta(1-z)\right], \\
P_{q g}(z)=\frac{1}{2}\left[z^{2}+(1-z)^{2}\right],
\end{gathered}
$$



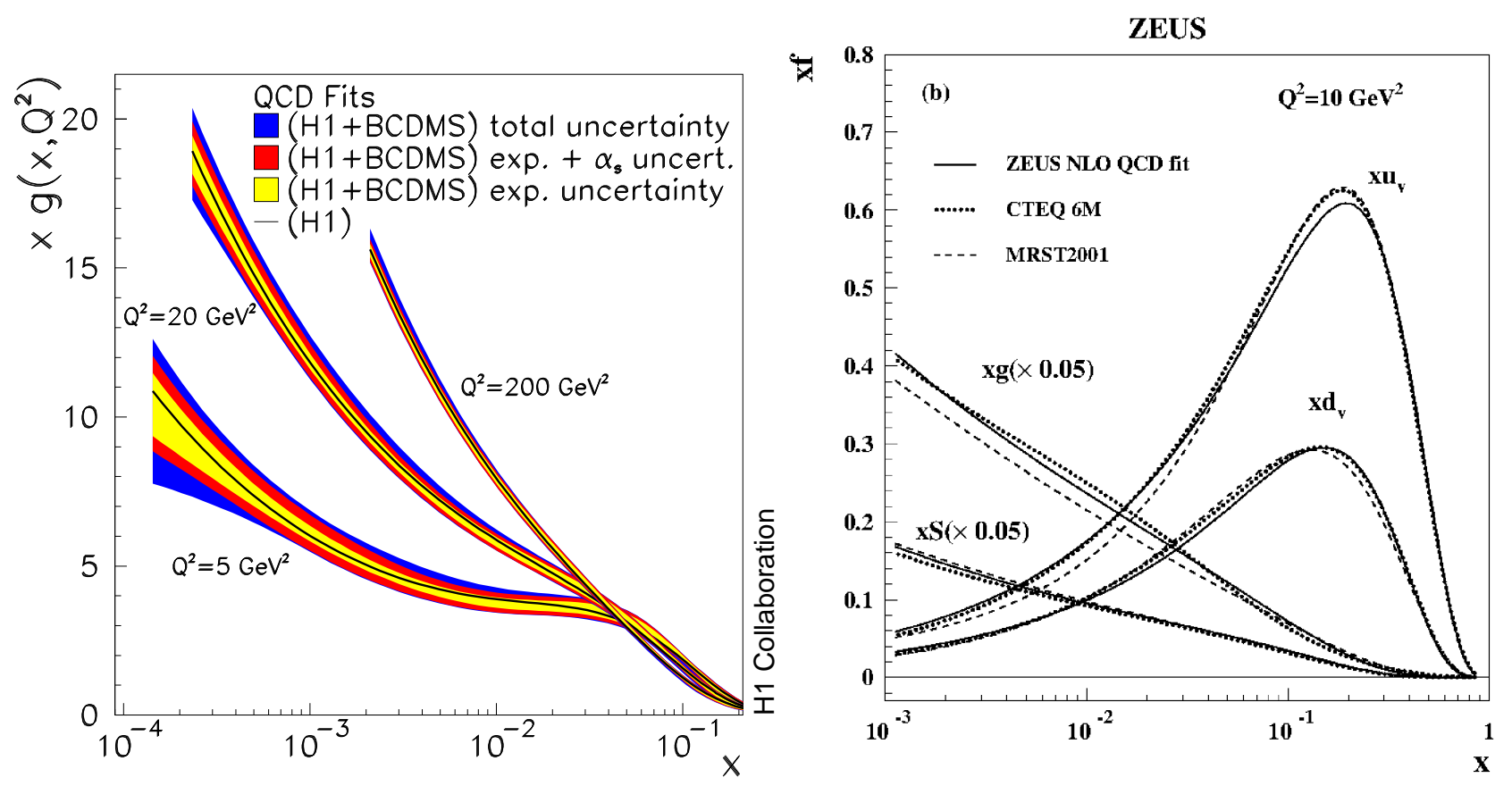

Fig. 2.7: PDFs extraídas de dados experimentais [20] e [21].

$$
\begin{gathered}
P_{g q}(z)=\frac{4}{3}\left[\frac{1+(1-z)^{2}}{z}\right], \\
P_{g g}(z)=6\left[\frac{(1-z)}{z}+\frac{z}{(1-z)_{+}}+\frac{33-2 n_{f}}{6} \delta(1-z)\right]
\end{gathered}
$$

com a definição

$$
\int_{0}^{1} \mathrm{~d} z \frac{f(z)}{(1-z)_{+}}=\int_{0}^{1} \mathrm{~d} z \frac{f(z)-f(1)}{1-z}
$$

\subsection{Análise global e obtenção das PDFs}

A chamada fatorização colinear, representada pela Eq.(2.62), nos permite escrever as seções de choque e a função de estrutura $F_{2}$ do DIS em termos das PDFs. De fato, pelas Eq.(2.39), (2.48) e (2.49), observamos que o conhecimento das PDFs é necessário para o cálculo de observáveis de interesse. As equações DGLAP nos fornecem a evolução das 
PDFs com $Q^{2}$, mas não com $x$. Poderíamos buscar parametrizações analíticas consistentes com a evolução em $Q^{2}$ produzido pela dinâmica DGLAP. Infelizmente, tais parametrizações analíticas não existem para todo o range de $x, Q^{2}$, especialmente quando vamos para ordens além da ordem dominante (NLO - next to leading order).

A forma com que o problema acima é normalmente atacado, se dá através das chamadas análises globais de um grande conjunto de dados do DIS e de outros processos hard (vários detalhes são discutidos na Ref.[11]). Abaixo, resumimos em que consiste esse método de obtenção das PDFs:

- uma forma analítica para as PDFs (quarks de valência, de mar e glúons) é assumida em um valor inicial arbitrário (contanto que seja perturbativo) de $Q_{0}^{2}$.

- as PDFs são evoluídas via dinâmica DGLAP para diferentes valores de $Q^{2}$.

- logo após, as PDFs são convoluídas com as funções coeficiente (ver Eq.(2.63)), onde deve ser escolhido o esquema de fatorização, a fim de se obter a função de estrutura $F_{2}$.

- um ajuste de parâmetros deve ser feito de forma que o resultado de $F_{2}$ descreva um grande conjunto de dados experimentais (análise global) referentes à diversos processos.

- as PDFs são fornecidas na forma de grids em $\left(x, Q^{2}\right)$. Esses grids nos fornecem o valor das PDFs em qualquer ponto $\left(x, Q^{2}\right)$ através de uma interpolação.

A Fig.2.7, mostra as PDFs extraídas de dados experimentais de H1 (à esquerda) e de ZEUS (à direita). No gráfico de H1, as bandas para uma mesma curva são referentes às incertezas do experimento. No gráfico de ZEUS, observamos uma comparação entre os modelos de diferentes grupos. Nos dois gráficos podemos observar um grande crescimento da função de distribuição de glúons $x g\left(x, Q^{2}\right)$ à medida que $x$ diminui. Além disso, podemos observar também que $x g\left(x, Q^{2}\right)$ é dominante frente às outas PDFs, no limite de pequeno $x$.

\subsection{Conclusão}

Nesse capítulo, introduzimos noções básicas da QCD como sua lagrangiana, as regras de Feynman, e sua constante de acoplamento variável com a escala. Além disso, apresentamos o espalhamento profundamente inelástico, que é uma importante ferramenta para o estudo da estrutura dos hádrons, e mostramos os principais resultados do modelo de pártons. Dentro do modelo de pártons definimos as funções de distribuição de pártons e as funções de estrutura. Mostramos ainda que esse modelo prevê o scaling de Bjorken. Logo após, discutimos a violação do scaling de Bjorken e como tal violação pode ser estudada levando em conta correções devidas à QCD, que nos conduzem às equações de evolução DGLAP, as quais implicam um forte crescimento de $x g\left(x, Q^{2}\right)$ no limite de pequeno $x$. No próximo capítulo, vamos estudar mais profundamente as características da dinâmica de altas energias (ou dinâmica de pequeno $x$ ). Para isso, vamos apresentar alguns dos principais métodos que nos permitem estudar processos de espalhamento em altas energias, bem como as implicações físicas nessas condições. 


\section{Capítulo 3}

\section{Dinâmica de pequeno $\mathrm{x}$}

No capítulo 2 estudamos como correções em $\ln Q^{2}$ surgem no modelo de pártons ao levarmos em conta os efeitos da QCD. Tais correções se tornam importantes para grande $Q^{2}$ e levam às equações de evolução DGLAP para as funções de distribuição de quarks e glúons. Além disso, observamos também que as PDFs extraídas de dados experimentais indicam um forte crescimento da distribuição de glúons no limite de pequeno $x$ (altas energias). Nesse capítulo estamos interessados no estudo da dinâmica de altas energias.

Quando consideramos espalhamentos em altas energias entre dois quarks, por exemplo, termos em $\alpha_{s} \ln 1 / x \approx 1$ aparecem em cálculos de amplitudes de espalhamento [18]. A princípio, isso poderia trazer problemas com relação à convergência da série perturbativa. Além disso, neste regime e com $Q^{2}$ moderado, as equações DGLAP deixam de ser válidas. Portanto, precisamos de mecanismos que ressomem termos em $\alpha_{s} \ln 1 / x$. Tais mecanismos levam às equações de evolução BFKL [22, 23] (devida a Balitsky, Fadin, Kuraev e Lipatov), GLR [24] (devida a Gribov, Levin e Ryskin) e BK [25, 26] (devida a Balitsky e Kovchegov), que são equações de evolução em $\ln 1 / x$.

Começaremos introduzindo o formalismo de dipolo (Refs.[27, 28, 29, 30]), que é uma importante ferramenta, amplamente utilizada nesta tese, para o cálculo de vários processos de espalhamento em pequeno $x$. Ele fornece uma descrição quântica do processo de espalhamento fóton-hádron bastante intuitiva. Além disso, com este formalismo os efeitos de saturação são levados em conta facilmente através da seção de choque de interação dipolo-hádron (ou seção de choque de dipolo), que carrega toda a informação das interações fortes para um dado processo. Definiremos neste capítulo a seção de choque de dipolo e mostraremos como ela se relaciona com as equações de evolução. Vamos apresentar também algumas características das equações de evolução no limite de pequeno $x$ e discutiremos a necessidade da saturação, que surge naturalmente da análise das soluções das equações de evolução lineares em altas energias e da imposição da unitariedade da matriz S. Logo após apresentamos a equação BK e suas características, bem como os modelos fenomenológicos utilizados nesta tese que descrevem a dinâmica do espalhamento devido à interação forte.

\subsection{Formalismo de Dipolo}

No formalismo de dipolo, uma colisão fóton-alvo é descrita da seguinte maneira: consi- 


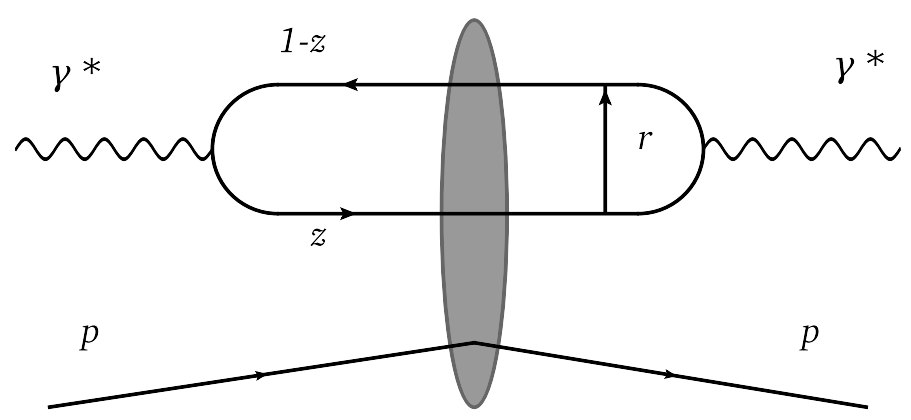

Fig. 3.1: Amplitude de espalhamento elástico $\gamma^{*} p \rightarrow \gamma^{*} p$ via formalismo de dipolo.

deramos um fóton virtual com grande momento longitudinal que irá colidir com um hádron em repouso (alvo). O fóton virtual flutua em um dipolo de cor (um par quark-antiquark) que espalha via interação forte com o hádron. Esta forma de pensar o espalhamento dipolohádron é válida no limite em que o tempo de interação, que é da ordem do tamanho do hádron, for muito menor do que o tempo de formação do dipolo, uma vez que nestas condições o tamanho do dipolo pode ser considerado fixo. Pode-se mostrar que o tempo de formação do dipolo é da ordem do inverso de $x[9,11,18]$. Portanto este formalismo é ideal para o estudo e processos em pequeno $x$.

Vamos agora apresentar características e resultados do formalismo de dipolo, segundo o qual, a amplitude elástica para o espalhamento $\gamma^{*} p$ ocorre em três etapas (ver Fig. 3.1):

- o fóton virtual flutua em um par $q \bar{q}$

- o par $q \bar{q}$ interage com o alvo

- o par $q \bar{q}$ se recombina formando um fóton virtual.

A amplitude para o espalhamento elástico $\gamma^{*} p \rightarrow \gamma^{*} p$, denotada por $\mathcal{A}^{\gamma^{*} p}(x, Q, \Delta)$, é dada pelo produto dos três subprocessos integrados sobre as variáveis do dipolo $\vec{r}$ e $z$ que são respectivamente a separação transversal do dipolo e a fração de momento longitudinal do fóton portada pelo quark [31] (ou, de maneira mais formal, no espaço de fase do cone de luz na representação mista: coordenada transversal e fração de momento longitudinal [9])

$$
\mathcal{A}^{\gamma^{*} p}(x, Q, \Delta)=\sum_{f} \sum_{h, \bar{h}} \int \mathrm{d}^{2} r \int_{0}^{1} \mathrm{~d} z \psi_{h \bar{h}}^{*}(r, z, Q) \mathcal{A}_{q \bar{q}}(x, r, \Delta) \psi_{h \bar{h}}(r, z, Q)
$$

onde $\psi_{h \bar{h}}(r, z, Q)$ é a função de onda do cone de luz do fóton virtual, que é a amplitude de probabilidade de um fóton flutuar num dipolo $q \bar{q}$ com helicidades $h$ e $\bar{h}$ e sabor $f$ (mais detalhes sobre as funções de onda podem ser encontrados no apêndice A). Além disso, $\mathcal{A}_{q \bar{q}}(x, r, \Delta)$ é a amplitude para o processo elementar do espalhamento de um dipolo de 
tamanho $\vec{r}$ com o próton. Por fim, $\Delta$ é o momento transversal perdido pelo próton emergente e $x$ é a variável de Bjorken.

A seção de choque do processo elementar é dada por [31]

$$
\frac{\mathrm{d} \sigma_{q \bar{q}}}{\mathrm{dt}}=\frac{1}{16 \pi}\left|\mathcal{A}_{q \bar{q}}(x, r, \Delta)\right|^{2}
$$

onde $t=-\Delta^{2}$. A amplitude $\mathcal{A}_{q \bar{q}}(x, r, \Delta)$ pode ser escrita como

$$
\mathcal{A}_{q \bar{q}}(x, r, \Delta)=\int \mathrm{d}^{2} \vec{b} e^{-i \vec{b} \cdot \vec{\Delta}} \mathcal{A}_{q \bar{q}}(x, r, b)
$$

Em termos da matriz $S, \mathcal{A}_{q \bar{q}}(x, r, b)$ pode ser parametrizada como [31]

$$
\mathcal{A}_{q \bar{q}}(x, r, b)=2 i[1-S(x, r, b)]
$$

levando a

$$
\mathcal{A}_{q \bar{q}}(x, r, \Delta)=i \int \mathrm{d}^{2} \vec{b} e^{-i \vec{b} \cdot \vec{\Delta}} 2[1-S(x, r, b)]
$$

O teorema óptico nos permite escrever [31, 32]

$$
\begin{aligned}
\sigma_{q \bar{q}}(x, r) & =\operatorname{Im}_{\mathcal{A}_{q \bar{q}}}(x, r, \Delta=0) \\
& =2 \int \mathrm{d}^{2} b[1-\operatorname{Re} S(x, r, b)] .
\end{aligned}
$$

A seção de choque total para o espalhamento $\gamma^{*} p$ é obtida usando a equação acima via teorema óptico

$$
\begin{aligned}
\sigma_{T, L}^{\gamma^{* p}}(x, Q) & =\operatorname{Im} \mathcal{A}_{T, L}^{\gamma^{*} p}(x, Q, \Delta=0) \\
& =\sum_{f} \int \mathrm{d}^{2} r \int_{0}^{1} \mathrm{~d} z\left[\psi^{*} \psi\right]_{T, L}^{f} \sigma_{q \bar{q}}(x, r) .
\end{aligned}
$$

Esta equação é a base do formalismo de dipolo [27, 28, 29, 30]. Ela nos mostra que a seção de choque total $\gamma^{*} p$ pode ser fatorizada como sendo o produto do quadrado da função de onda do cone de luz (para o splitting do fóton virtual em um par $q \bar{q}$ ) pela seção de choque do processo elementar $q \bar{q} p$.

A seguir, listamos a forma como $\sigma_{d i p}$ se relaciona com grandezas físicas de interesse:

- A seção de choque de dipolo pode ser escrita em termos da função de distribuição de glúons da seguinte forma [18]

$$
\sigma_{d i p}(x, r) \approx \frac{\pi^{2}}{3} r^{2} \alpha_{s}(r) x g\left(x, B / r^{2}\right)
$$

onde $B \approx 10$ e $g$ satisfaz a dinâmica DGLAP. A equação acima é válida na aproximação de duplo logaritmo dominante (isto é, no limite em que $\ln Q^{2}, \ln 1 / x \rightarrow \infty$ ). Podemos observar ainda que $\sigma_{d i p} \rightarrow 0$ no limite em que $r \rightarrow 0$. Esse é o chamado limite de transparência de cor, para o qual o dipolo não interage via interação forte. 
- Vamos definir a distribuição de glúons não integrada $f$, a qual está associada com $g\left(x, Q^{2}\right)$ por

$$
x g\left(x, Q^{2}\right)=\int^{Q^{2}} \frac{\mathrm{d} k_{\perp}^{2}}{k_{\perp}^{2}} f\left(x, k_{\perp}^{2}\right)
$$

Como veremos mais adiante, esta quantidade satisfaz a equação BFKL. A seção de choque de dipolo se relaciona com $f\left(x, k_{\perp}^{2}\right)$ através de $[11,18]$

$$
\sigma(x, r)=\frac{4 \pi}{3} \int \frac{\mathrm{d}^{2} \vec{k}}{\vec{k}^{4}} \alpha_{s} f\left(x, \vec{k}^{2}\right)\left(1-e^{i \vec{k} \cdot \vec{r}}\right)
$$

onde $|\vec{k}|=k_{\perp}$. A relação acima surge naturalmente derivando-se o formalismo de dipolo com o auxílio da fatorização $\vec{k}_{\perp}[18]$.

- A Eq. (3.6) pode ser escrita como

$$
\begin{aligned}
\sigma_{q \bar{q}}(x, r) & =2 \int \mathrm{d}^{2} b[1-\operatorname{Re} S(x, r, b)] \\
& =2 \int \mathrm{d}^{2} b \mathcal{N}(x, r, b) .
\end{aligned}
$$

No segundo sinal de igualdade definimos a parte imaginária da amplitude de espalhamento frontal dipolo-alvo $\mathcal{N}$. Este é um dos principais ingredientes desta tese, para o qual utilizamos alguns modelos. Assim como $\sigma_{\text {dip }}, \mathcal{N}$ carrega toda a informação sobre a interação via força forte entre o dipolo e alvo hadrônico. Nas próximas seções mostraremos como $\mathcal{N}$ se relaciona com algumas das equações de evolução da QCD em altas energias.

\subsection{Solução das equações DGLAP no limite de duplo logaritmo dominante}

Analisando as funções de splitting das Eqs. DGLAP para pequeno $x(z \rightarrow 0)$, obtemos (ver as Eqs. 2.71 - 2.74 do capítulo anterior)

$$
P_{q q}(z) \rightarrow \frac{4}{3} ; P_{q g}(z) \rightarrow \frac{1}{2} ; P_{g q}(z) \rightarrow \frac{8}{3 z} ; P_{g g}(z) \rightarrow \frac{6}{z}
$$

Observamos que as funções de splitting $P_{g q}$ e $P_{g g}$ são singulares enquanto as $P_{q q}$ e $P_{q g}$ não. Portanto a evolução da QCD em pequeno $x$ é dominada por glúons, conforme discutido no final do capítulo anterior. Considerando apenas o setor de glúons $g\left(x, Q^{2}\right)$, podemos aproximar a equação DGLAP por

$$
\frac{\partial g(x, t)}{\partial t}=\frac{\alpha_{s}(t)}{2 \pi} \int_{x}^{1} \frac{\mathrm{d} \xi}{\xi} P_{g g}\left(\frac{x}{\xi}\right) g(\xi, t)
$$


que pode ser reescrito como

$$
\frac{\partial g(x, t)}{\partial t}=\frac{\alpha_{s}(t)}{2 \pi} \int_{0}^{\ln \left(\frac{1}{x}\right)} \mathrm{d} \ln \left(\frac{1}{\xi}\right) P_{g g}\left(\frac{x}{\xi}\right) g(\xi, t) .
$$

Aproximando $P_{g g}$ pela Eq. (3.12) (usando $\left.P(x / \xi)=6 \xi / x\right)$ e substituindo a expressão da constante de acoplamento (em mais baixa ordem é $\alpha_{s}=1 /\left(b_{0} t\right)$ ), obtemos

$$
t \frac{\partial x g(x, t)}{\partial t}=\frac{3}{\pi b_{0}} \int_{0}^{\ln \left(\frac{1}{x}\right)} \mathrm{d} \ln \left(\frac{1}{\xi}\right) \xi g(\xi, t) .
$$

Definindo agora

$$
u=\ln \left(\frac{t}{t_{0}}\right) ; v=\ln \left(\frac{x_{0}}{x}\right)
$$

e $G(u, v)=x g(x, t)$ a Eq. (3.14) se reduz a

$$
\frac{\partial^{2} G(u, v)}{\partial u \partial v}=\gamma^{2} G(u, v), \operatorname{com} \gamma^{2}=\frac{3}{\pi b_{0}} .
$$

Para encontrar a solução do limite duplamente assintótico $\left(\ln Q^{2}, \ln (1 / x) \rightarrow \infty\right)$, é conveniente fazermos a seguinte mudança de variáveis

$$
\rho=\left(\frac{v}{u}\right)^{\frac{1}{2}}, \sigma=(u v)^{\frac{1}{2}}
$$

O limite que estamos estudando se traduz em $\sigma \rightarrow \infty$ e $\rho$ fixo. Com as transformações acima, temos a seguinte relação

$$
4 \frac{\partial^{2}}{\partial u \partial v}=\frac{\partial^{2}}{\partial \sigma^{2}}-\frac{\rho}{\sigma^{2}} \frac{\partial}{\partial \rho}+\frac{1}{\sigma} \frac{\partial}{\partial \sigma}-\frac{\rho^{2}}{\sigma^{2}} \frac{\partial^{2}}{\partial \rho^{2}},
$$

que desprezando os termos proporcionais a $1 / \sigma($ pois $\sigma \rightarrow \infty)$ se reduz a

$$
\frac{\partial^{2}}{\partial u \partial v}=\frac{1}{4} \frac{\partial^{2}}{\partial \sigma^{2}}
$$

Então a Eq. (3.16) pode ser escrita como

$$
\frac{\partial^{2} G(\sigma, \rho)}{\partial \sigma^{2}}=4 \gamma^{2} G(\sigma, \rho)
$$

cuja solução é dada por

$$
G(\sigma, \rho)=A \exp (2 \gamma \sigma)
$$

onde $A$ é uma constante. Escrevendo em termos das variáveis originais

$$
x g(x, t)=A \exp \left\{\sqrt{\frac{12}{\pi b_{0}} \ln \frac{t}{t_{0}} \ln \frac{x_{0}}{x}}\right\} .
$$

Por fim, seguindo o mesmo raciocínio, mas tomando $\alpha_{s}$ fixo, obtemos

$$
x g\left(x, Q^{2}\right)=A \exp \left\{2 \sqrt{\frac{3 \alpha_{s}}{\pi} \ln \frac{Q^{2}}{Q_{0}^{2}} \ln \frac{x_{0}}{x}}\right\}
$$

onde $Q^{2} \gg Q_{0}^{2}$. Essas são as soluções da equação DGLAP na aproximação de duplo logaritmo dominante. Podemos observar que essas soluções apresentam um crescimento rápido à medida em que $x$ se torna muito pequeno. 


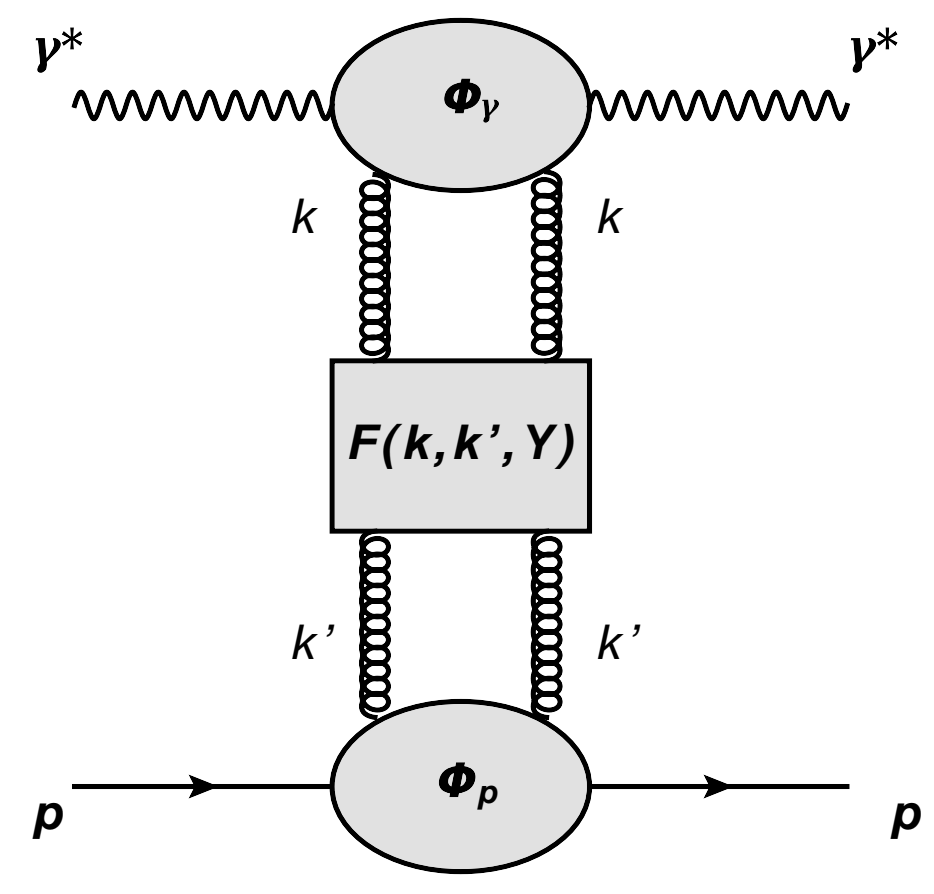

Fig. 3.2: Amplitude elástica para o espalhamento entre um fóton virtual e um próton em altas energias.

\subsection{Equação BFKL}

Conforme mencionado na introdução deste capítulo, para espalhamentos em altíssimas energias ( $x$ muito pequeno) e $Q^{2}$ moderado, a aproximação de duplo logaritmo dominante deixa de ser válida e encontramos termos da forma $\alpha_{s} Y \approx 1$, onde em altas energias, a variável de rapidez $Y$ é dada por $Y=\ln (1 / x)$. Devemos portanto considerar mecanismos que ressomem estes termos. Como discutiremos, tal ressoma é feita pela dinâmica BFKL.

Vamos considerar o espalhamento entre um fóton virtual e um próton. Conforme havia sido adiantado, em pequeno $x$ observamos um grande crescimento na densidade de glúons dentro do próton. Desta forma, os processos elementares $\gamma^{*} g$, mostrados nos dois últimos diagramas da Fig. 2.6 se tornam muito importantes para o espalhamento $\gamma^{*} p$. Além disso, em mais baixa ordem, os dois últimos diagramas da Fig. 2.6 fornecem a amplitude para o espalhamento $\gamma^{*} p$, com presença de um único glúon.

A dinâmica BFKL, considera correções devido às múltiplas emissões de glúons em altas energias. A Fig. 3.2, mostra o módulo quadrado da amplitude do espalhamento $\gamma^{*} p$ no 
contexto da dinâmica BFKL. A função $F$ presente na figura representa a escada gluônica perturbativa, responsável pelas correções em $\ln (1 / x)$ e as funções $\Phi_{\gamma}$ e $\Phi_{p}$ são, respectivamente, os fatores de impacto do fóton virtual e do próton e determinam o acoplamento da escada com as partículas externas. Além disso $F$ e $\Phi_{\gamma}$ podem ser calculadas perturbativamente, enquanto $\Phi_{p}$ é uma quantidade não perturbativa e só pode ser determinada de maneira fenomenológica.

No limite de altas energias, a seção de choque de espalhamento $\gamma^{*} p$ pode ser escrita como [18]

$$
\sigma_{\lambda}^{\gamma^{*} p}\left(x, Q^{2}\right)=\frac{1}{(2 \pi)^{4}} \int \frac{\mathrm{d}^{2} \vec{k}}{\vec{k}^{2}} \int \frac{\mathrm{d}^{2} \vec{k}^{\prime}}{\vec{k}^{\prime 2}} \Phi_{\lambda}\left(\vec{k}^{2}, Q^{2}\right) \Phi_{p}\left(\vec{k}^{\prime 2}\right) F\left(x, \vec{k}, \vec{k}^{\prime}\right),
$$

sendo $\Phi_{\lambda}=\left[\Phi_{\gamma}\right]_{\lambda}$ onde $\lambda$ explicita a polarização do fóton. Vamos definir a distribuição de glúons não integrada $f\left(x, \vec{k}^{2}\right)$ em termos de $F$

$$
f\left(x, \vec{k}^{2}\right)=\frac{1}{(2 \pi)^{3}} \int \frac{\mathrm{d}^{2} \vec{k}^{\prime}}{\vec{k}^{\prime 2}} \Phi_{p}\left(\vec{k}^{\prime}\right) \vec{k}^{2} F\left(x, \vec{k}, \vec{k}^{\prime}\right),
$$

de forma que a Eq. (3.24) possa ser reescrita da seguinte forma

$$
\sigma_{\lambda}^{\gamma^{*} p}\left(x, Q^{2}\right)=\frac{1}{2 \pi} \int \frac{\mathrm{d}^{2} \vec{k}}{\vec{k}^{4}} f\left(x, \vec{k}^{2}\right) \Phi_{\lambda}\left(\vec{k}^{2}, Q^{2}\right),
$$

isto é, a seção de choque passa a ser fatorizada entre uma quantidade não perturbativa $(f)$ e em uma perturbativa $(\Phi)$. Devido à definição da distribuição de glúons não integrada via Eq. (3.25), podemos observar que como $F$ satisfaz a dinâmica BFKL, então $f$ também irá satisfazer. Podemos então escrever a equação BFKL para a distribuição de glúons não integrada como $[9,18]$

$$
\frac{\partial f\left(x, k_{\perp}^{2}\right)}{\partial \ln (1 / x)}=\frac{\bar{\alpha}_{s}}{\pi} \int \frac{\mathrm{d}^{2} \vec{q}_{\perp}}{\left(\vec{k}_{\perp}-\vec{q}_{\perp}\right)^{2}}\left[f\left(x, q_{\perp}^{2}\right)-\frac{k_{\perp}^{2}}{2 q_{\perp}^{2}} f\left(x, k_{\perp}^{2}\right)\right] \quad, \quad \bar{\alpha}_{s}=\frac{\alpha N_{c}}{\pi} .
$$

A equação acima ressoma todas as correções de altas energias (em $\ln (1 / x)$ dominante) ao espalhamento $\gamma^{*} p$ em mais baixa ordem.

Consideremos duas soluções da equação BFKL. Seja uma escala $\Lambda$ de momento transversal inicial. É possível mostrar que para $k_{\perp} \sim \Lambda$, a equação BFKL fornece a seguinte solução $[9,18]$

$$
f\left(x, k_{\perp}^{2}\right) \sim\left(\frac{1}{x}\right)^{\alpha_{\mathbb{P}}-1} \exp \left\{-\frac{\ln ^{2}\left(k_{\perp} / \Lambda\right)}{14 \zeta(3) \bar{\alpha}_{s} \ln (1 / x)}\right\},
$$

sendo $\alpha_{\mathbb{P}}-1=4 \bar{\alpha}_{s} \ln 2$. Esta é a chamada aproximação de difusão e nos mostra que a distribuição de glúons não integrada cresce com uma potência de $1 / x$ em pequeno $x$. Outra solução pode ser obtida considerando-se $k_{\perp} \gg \Lambda$. Neste caso a solução é dada por $[9,18]$

$$
f\left(x, k_{\perp}^{2}\right) \sim \exp \left\{2 \sqrt{\bar{\alpha}_{s} \ln \left(\frac{1}{x}\right) \ln \left(\frac{k_{\perp}^{2}}{\Lambda^{2}}\right)}\right\},
$$


a qual é a versão da aproximação de duplo logaritmo dominante da BFKL. No apêndice B, estas soluções são derivadas em mais detalhes no espaço das posições transversais. Por fim, vamos fazer alguns comentários com respeito à estas soluções. Podemos observar que estas duas aproximações predizem um forte crescimento da distribuição de glúons não integrada (da mesma forma que a solução da equação DGLAP na aproximação de duplo logaritmo dominante, estudada na seção anterior). Como veremos na próxima seção, este comportamento viola o vínculo de unitariedade da matriz $S$. Portanto algum novo efeito deve ser levado em conta, de forma que tal violação não ocorra.

\subsection{Limite de Froissart-Martin}

A mecânica quântica impõe um limite máximo de crescimento da seção de choque. Ele é chamado de limite de disco negro e é uma consequência da unitariedade da matriz $S$, sendo expresso por [18]

$$
\sigma_{t o t} \leq 2 \pi R^{2}
$$

onde $R$ é o raio do disco negro.

Vamos considerar a seção de choque hádron-hádron num certo parâmetro de impacto $b$ tal que o limite de disco negro não seja alcançado. O espalhamento se dá pela troca de partículas e a seção de choque cresce com alguma potência positiva $\Delta$ da energia $\left(\sigma_{t o t} \sim s^{\Delta}\right)$. Por outro lado, a intensidade da interação deve cair com o aumento de $b$. A queda de probabilidade de interação mais lenta possível em QCD é

$$
e^{-2 m_{\pi} b}
$$

sendo $m_{\pi}$ a massa do píon (o qual é o estado ligado mais leve da QCD).

A probabilidade de interação $p$ deve ser da ordem de

$$
p \sim s^{\Delta} e^{-m_{\pi} b} .
$$

Se $p \sim 1$, a interação é intensa e esperamos que o comportamento de disco negro comece a se manifestar.

Temos que $p \approx 1$ para um dado parâmetro de impacto $b=b^{*}$, tal que

$$
s^{\Delta} e^{-2 m_{\pi} b^{*}}=1
$$

e

$$
R=b^{*} \sim \frac{\Delta}{2 m_{\pi}} \ln s
$$

Como $b^{*}$ é o valor de $b$ onde o limite de disco negro se manifesta, temos

$$
\sigma_{t o t} \leq 2 \pi R^{2}=\frac{\pi \Delta^{2}}{2 m_{\pi}^{2}} \ln ^{2} s
$$


A equação acima é o chamado limite de Froissart-Martin [33, 34]. Dela concluímos que, em QCD, as seções de choque não podem crescer mais rápido do que $\ln ^{2} s$. Aqui fizemos uma derivação extremamente simplificada. Uma derivação mais rigorosa (que pode ser encontrada na Ref.[18]), mostra que este limite surge, essencialmente, do requerimento de unitariedade.

Nas soluções da BFKL para a aproximação de difusão e duplo logaritmo dominante (bem como as soluções da DGLAP em pequeno $x$ ) estudadas anteriormente, vemos que elas implicam na violação do limite acima tendo em vista que crescem com potências da energia, o que é muito mais rápido do que o permitido pelo limite de Froissart-Martin. Nas próximas seções, vamos apresentar equações que não apresentem este problema.

\subsection{Equação GLR e efeitos de saturação}

Vamos determinar a região cinemática onde a BFKL viola a unitariedade. Seja um espalhamento onium-onium (isto é, um espalhamento entre dois mésons pesados). A dinâmica BFKL na aproximação de difusão fornece $\left(\operatorname{com} l_{\perp} \sim 1 / x_{\perp}\right)[9]$

$$
\sigma_{\text {tot }}^{\text {onium }+ \text { onium }} \sim \alpha_{s}^{2} x_{1 \perp} x_{2 \perp} e^{\left(\alpha_{P}-1\right) Y} .
$$

Devido ao limite de disco negro, temos que $\sigma_{\text {tot }}^{\text {onium }+ \text { onium }} \leq 2 \pi R^{2}$. Vamos fixar $R \sim r_{h}$, onde $r_{h}$ é o raio hadrônico.

Consideramos que um onium (um par quark - antiquark) vem do decaimento de um fóton virtual e que o outro desempenha o papel de hádron alvo. Logo $x_{1 \perp} \sim 1 / Q$ e $x_{2 \perp} \sim r_{h}$. Impondo o limite de disco negro

$$
\alpha_{s}^{2} \frac{r_{h}}{Q} e^{\left(\alpha_{P}-1\right) Y} \leq 2 \pi r_{h}^{2}
$$

A igualdade (o valor máximo do lado esquerdo) é alcançada para um certo valor $Q=Q_{s}$ tal que (lembrando que $Y=\ln (1 / x)$ )

$$
\begin{aligned}
\frac{\alpha_{s}^{2}}{Q_{s}} e^{\ln (1 / x)^{\alpha_{P}-1}} & \sim \frac{1}{\Lambda_{Q C D}} \\
\rightarrow Q_{s} & \sim \alpha_{s}^{2} \Lambda_{Q C D}\left(\frac{1}{x}\right)^{\alpha_{p}-1}
\end{aligned}
$$

onde tomamos $r_{h} \sim 1 / \Lambda_{Q C D}$. Concluímos, portanto, que a violação da unitariedade ocorre para valores $Q<Q_{s}$. Para $x$ muito pequeno, temos $Q_{s} \gg \Lambda_{Q C D}$, o que implica que a violação da unitariedade começa para pequenas distâncias $\left(\sim 1 / Q_{s}\right)$, ainda no domínio da QCD perturbativa (pQCD). Então o problema da BFKL violar a unitariedade deve ser resolvido na pQCD. Conforme $x$ diminui, a densidade de pártons aumenta e para um dado valor de $x$, a densidade é tão grande que as funções de onda dos pártons passam a se sobrepor. Para um sistema densamente populado, devemos esperar a interação entre os pártons. Portanto, para $Q^{2} \leq Q_{s}^{2}$ devemos ter uma nova equação que inclui, por exemplo, 
a recombinação de dois glúons em um. Esta equação deve diminuir e, até mesmo, parar o crescimento do número de pártons.

Devemos ressaltar que como em altas energias $Q_{s}^{2} \gg \Lambda_{Q C D}^{2}, \alpha_{s}$ é pequeno e os métodos de QCD perturbativa podem ser aplicados.

Gribov, Levin e Ryskin [24] propuseram que antes da energia se tornar alta o suficiente para que houvesse uma violação do limite de Froissart, poderia existir uma região de energia intermediária, onde a física da distribuição de glúons é dominada pela fusão de dois glúons em um, que traria um termo não linear para a equação BFKL [24]

$$
\frac{\partial f\left(x, k_{\perp}^{2}\right)}{\partial \ln (1 / x)}=\frac{\alpha_{s} N_{c}}{\pi^{2}} \int \mathrm{d}^{2} l_{\perp} K_{B F K L}(k, l) f\left(x, k_{\perp}^{2}\right)-\frac{\alpha_{s}^{2} N_{c} \pi}{2 C_{F} S_{\perp}}\left[f\left(x, k_{\perp}^{2}\right)\right]^{2},
$$

sendo a notação

$$
\int \mathrm{d}^{2} l_{\perp} K_{B F K L}(k, l) f\left(x, k_{\perp}^{2}\right)=\int \frac{\mathrm{d}^{2} l_{\perp}}{\left(\vec{k}_{\perp}-\vec{l}_{\perp}\right)^{2}}\left[f\left(x, l_{\perp}^{2}\right)-\frac{k_{\perp}^{2}}{2 l_{\perp}^{2}} f\left(x, k_{\perp}^{2}\right)\right]
$$

e $S_{\perp}=\pi R^{2}$ a área da seção transversal do próton. O termo quadrático é responsável pela fusão de dois glúons. Ele leva a um amortecimento no crescimento das funções de distribuição de glúons com a energia. Este fenômeno é conhecido como saturação das distribuições de pártons.

Mueller e Qiu (Ref.[35]) derivaram a equação GLR na aproximação de duplo logaritmo dominante para a função de distribuição de glúons integrada,

$$
x g\left(x, Q^{2}\right)=\int^{Q^{2}} \frac{\mathrm{d} k_{\perp}^{2}}{k_{\perp}^{2}} f\left(x, k_{\perp}^{2}\right),
$$

dada por

$$
\frac{\partial^{2} x g\left(x, Q^{2}\right)}{\partial \ln (1 / x) \partial \ln \left(Q^{2} / \Lambda^{2}\right)}=\frac{\alpha_{s} N_{c}}{\pi} x g\left(x, Q^{2}\right)-\frac{\alpha_{s}^{2} N_{c} \pi}{2 C_{F} S_{\perp}} \frac{1}{Q^{2}}\left[x g\left(x, Q^{2}\right)\right]^{2} .
$$

Se impomos a saturação de $x g\left(x, Q^{2}\right)$ (isto é, o lado esquerdo da equação acima igual a zero), obtemos

$$
\begin{aligned}
Q_{s}^{2} & =\frac{\alpha_{s} \pi^{2}}{S_{\perp} 2 C_{F}} x g\left(x, Q^{2}\right) \\
& =\frac{\alpha_{s} \pi^{2}}{S_{\perp} 2 C_{F}}\left(\frac{1}{x}\right)^{\lambda}
\end{aligned}
$$

onde, da aproximação de difusão (Eq. 3.28)

$$
x g\left(x, Q^{2}\right) \sim\left(\frac{1}{x}\right)^{\lambda}
$$

Para um núcleo com A núcleons, devemos multiplicar a Eq. (3.43) por $A$

$$
Q_{s}^{2} \sim \frac{A}{S_{\perp}}\left(\frac{1}{x}\right)^{\lambda} \sim A^{1 / 3}\left(\frac{1}{x}\right)^{\lambda}
$$

pois $S_{\perp} \sim R^{2}$ e $R \sim A^{1 / 3}$. O fator de $A^{1 / 3}$ na equação acima nos diz que $Q_{s}^{2}$ é maior para núcleos. Como efeitos não lineares (efeitos de saturação) são importantes para $Q^{2} \lesssim Q_{s}^{2}$, a região de saturação é mais ampla para DIS com núcleos pesados (grande $A$ ). Isso também fornece uma forte justificativa para o uso de pQCD. 


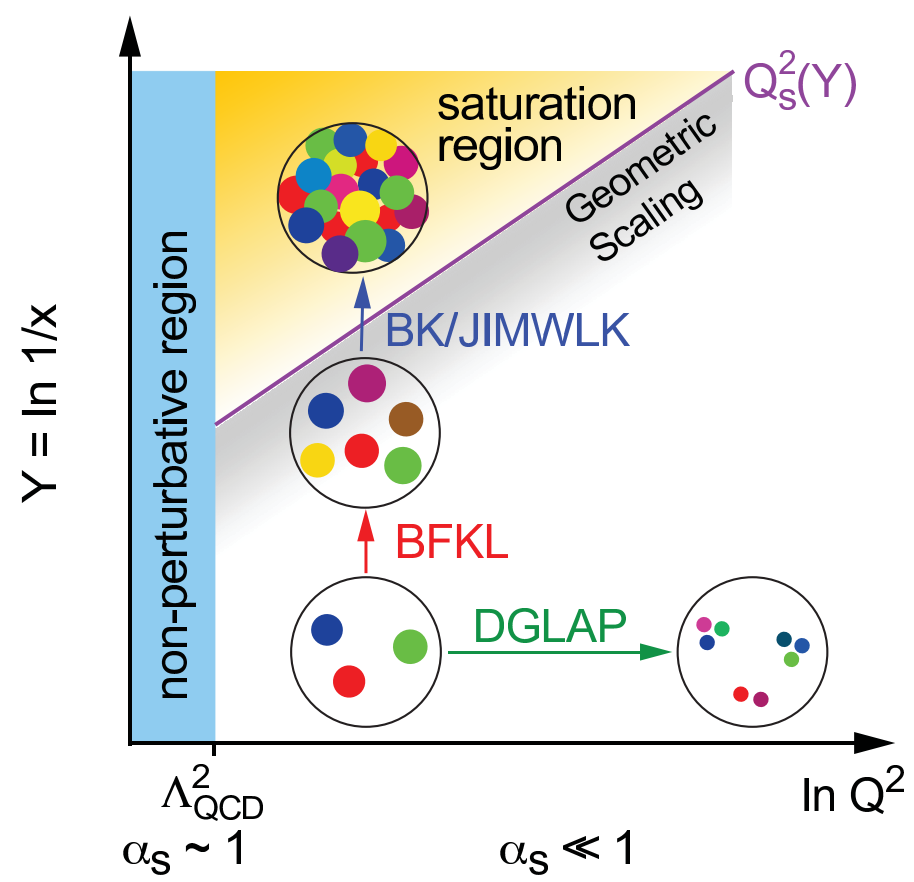

Fig. 3.3: Diagrama $\left(x, Q^{2}\right)$ para a $Q C D$ em altas energias. Aqui $Q_{s}$ é a escala de saturação. A figura foi retirada da Ref.[36].

\subsection{Saturação no formalismo de dipolo de cor}

$\mathrm{Na}$ seção anterior introduzimos o fenômeno de saturação. Tendo em vista que o nosso formalismo básico para o estudo da interação $\gamma^{*} p(A)$ é o formalismo de dipolo de cor, vamos tentar entender como ele se relaciona com o fenômeno da saturação.

A Fig. 3.3 é útil para ilustrar como um espalhamento $\gamma^{*}$-hádron pode ser entendido. Nela, os eixos evoluem o hádron em $\ln (1 / x)$ e em $\ln Q^{2}$ e a linha $Q_{s}(Y=\ln 1 / x)$ descreve, de forma qualitativa, a escala de saturação, a qual separa os regimes linear e não linear (isto é, onde espera-se que efeitos de saturação sejam importantes). Vamos sintetizar as principais informações fornecidas por este diagrama:

- inicialmente vamos tomar o caso em que $x$ é constante enquanto evoluímos para valores de $Q^{2}$ maiores. Conforme já estudado, esta evolução é regida pela dinâmica DGLAP. Neste caso esperamos um crescimento da densidade de glúons dentro do hádron. Por outro lado, aumentando $Q^{2}$, aumentamos a resolução com a qual o fóton virtual sonda o hádron. Tal aumento pode ser entendido via formalismo de dipolo da seguinte forma: inicialmente, o fóton virtual flutua em um par $q \bar{q}$ com separação transversal $r$. Neste 
caso, o dipolo resolve ("enxerga") pártons com dimensões transversais da ordem de $r$. Como $r \sim 1 / Q$, ao aumentarmos a virtualidade, diminuímos $r$ e, consequentemente, as dimensões transversais dos pártons. Neste caso, o sistema evolui para um sistema diluído.

- vamos agora considerar a virtualidade constante enquanto vamos para valores de $x$ cada vez menores (isto é, para energias cada vez maiores). Neste caso, conforme estudado pela equação BFKL, esperamos o crescimento da densidade de glúons dentro do hádron. Temos que $Q^{2}$ e consequentemente $r$ são constantes. Desta forma, em uma energia suficientemente alta, os glúons passam a se sobrepor, tornando o hádron um sistema de alta densidade. Para energias suficientemente altas e $Q^{2}<Q_{s}^{2}$ (neste caso, os dipolos possuem $r$ grande quando comparado com $1 / Q_{s}$ ), espera-se que efeitos da física de saturação comecem a se manifestar. Assim sendo, devemos buscar outras equações de evolução que levem em conta tais efeitos.

- por completeza, evoluindo o sistema para $x$ pequeno e alto $Q^{2}$, atingimos a aproximação de duplo logaritmo dominante.

O sistema de alta densidade partônica formado em altas energias para $Q^{2}<Q_{s}^{2}$ é chamado na literatura de Color Glass Condensate (denotado por CGC) [9, 11, 37]. Na teoria efetiva do CGC [38, 39, 40], considera-se que ocorram múltiplas emissões de glúons soft (pequeno $x$ ) por fontes que se movem rapidamente (glúons com $x^{\prime} \gg x$ ). Estes campos são descritos por um campo clássico (com grande número de ocupação) de Yang-Mills. De fato, é possível mostrar que o tempo de vida de um párton é proporcional à sua fração de momentum $x$ (Ref.[41]), de forma que glúons "lentos" possuem um tempo de vida muito menor do que os glúons "rápidos" (com fração de momentum $x^{\prime}$ ). Portanto, para a dinâmica de pequeno $x$, considera-se que glúons com $x^{\prime} \gg x$ atuam como fontes de cor congeladas (estacionárias), as quais emitem glúons na escala $x$. Esta teoria possui semelhanças técnicas com a teoria de vidros de spin, onde ocorre a separação dos graus de liberdade de acordo com as escalas de tempo [9]. Podemos acrescentar a este aspecto o fato dos glúons portarem cor e serem bósons os quais formam condensados no regime de altas densidades. Estas características motivam o nome CGC.

A teoria do CGC fornece uma equação de evolução não linear em $\ln (1 / x)$, chamada de equação JIMWLK (devida a Jalilian-Marian, Iancu, McLerran, Weigert, Leonidov e Kovner). Esta equação representa uma hierarquia infinita de equações acopladas as quais descrevem a evolução simultânea de todas as funções de correlação da teoria [41]. É possível mostrar que no limite de grande $N_{c}$, esta equação leva à equação BK, a qual será discutida na próxima seção (maiores detalhes podem ser encontrados na Ref.[9]).

\subsection{Equação BK}

Nesta seção, vamos apresentar a equação BK e suas características. Esta equação, assim como a equação BFKL, ressoma termos em $\alpha_{s} \ln 1 / x$. Entretanto, como veremos, ela leva em conta efeitos não lineares. Inicialmente consideremos a Fig. 3.4, onde no estado inicial temos 


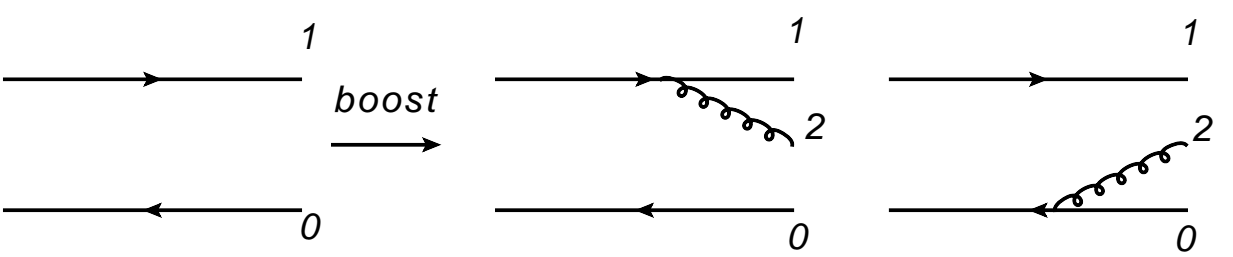

Fig. 3.4: Dipolo de cor inicial e depois de um passo de evolução em rapidez.

um dipolo de cor. Após um passo em rapidez, devemos levar em conta correções devido à emissão de um glúon pelo dipolo. Com base na Fig. 3.4, podemos definir as posições transversais ocupadas por cada partícula do par e do glúon: o quark ocupa a posição $x_{1}$, o antiquark ocupa a posição $x_{0}$ e o glúon ocupa a posição $x_{2}$. Definimos também $x_{i j}=\left|\vec{x}_{i}-\vec{x}_{j}\right|$, a fim de tornar nossa notação mais compacta.

Queremos, portanto, estudar a dependência da amplitude de espalhamento com a rapidez (energia). Um cálculo completo consistiria em analisar o comportamento da função de onda do dipolo antes e depois das emissões de um glúon real e um virtual (como feito, por exemplo, nas Refs. [9, 42, 43, 44]). Aqui, apresentaremos apenas um possível caminho à equação BK. Temos que em um passo de rapidez, a amplitude para o espalhamento $q \bar{q}$ com o alvo é $[43,44]$

$$
\mathcal{N}_{q \bar{q}}\left(Y+\Delta Y, \vec{x}_{10}\right)=\mathcal{N}_{q \bar{q}}\left(Y, \vec{x}_{10}\right)+\mathcal{O}\left(\alpha_{s} \ln Y\right)
$$

onde $\mathcal{O}\left(\alpha_{s}\right)$ caracteriza as correções devido às emissões de glúons. Seguindo [43, 44], temos que as correções devido às emissões reais e virtuais são, respectivamente,

$$
\frac{\alpha_{s} C_{F}}{\pi^{2}} \int \mathrm{d} Y \mathrm{~d} x_{2} \frac{x_{10}^{2}}{x_{20}^{2} x_{21}^{2}} \mathcal{N}_{q \bar{q} g}\left(\vec{x}_{10}, \vec{x}_{20}\right)
$$

e

$$
-\frac{\alpha_{s} C_{F}}{\pi^{2}} \int \mathrm{d} Y \mathrm{~d} x_{2} \frac{x_{10}^{2}}{x_{20}^{2} x_{21}^{2}} \mathcal{N}_{q \bar{q}}\left(\vec{x}_{10}\right)
$$

Então a Eq. (3.46) fica

$$
\begin{aligned}
\mathcal{N}_{q \bar{q}}\left(Y+\Delta Y, \vec{x}_{10}\right)=\mathcal{N}_{q \bar{q}}\left(Y, \vec{x}_{10}\right) & +\frac{\alpha_{s} C_{F}}{\pi^{2}} \int \mathrm{d} Y \mathrm{~d} x_{2} \frac{x_{10}^{2}}{x_{20}^{2} x_{21}^{2}} \\
& \times\left[\mathcal{N}_{q \bar{q} g}\left(\vec{x}_{10}, \vec{x}_{20}, Y\right)-\mathcal{N}_{q \bar{q}}\left(\vec{x}_{10}, Y\right)\right] .
\end{aligned}
$$

No limite de grande $N_{c}$, a emissão de um glúon, pode ser interpretada como a emissão de um dipolo. Desta forma teremos um novo sistema, agora com dois dipolos, como 


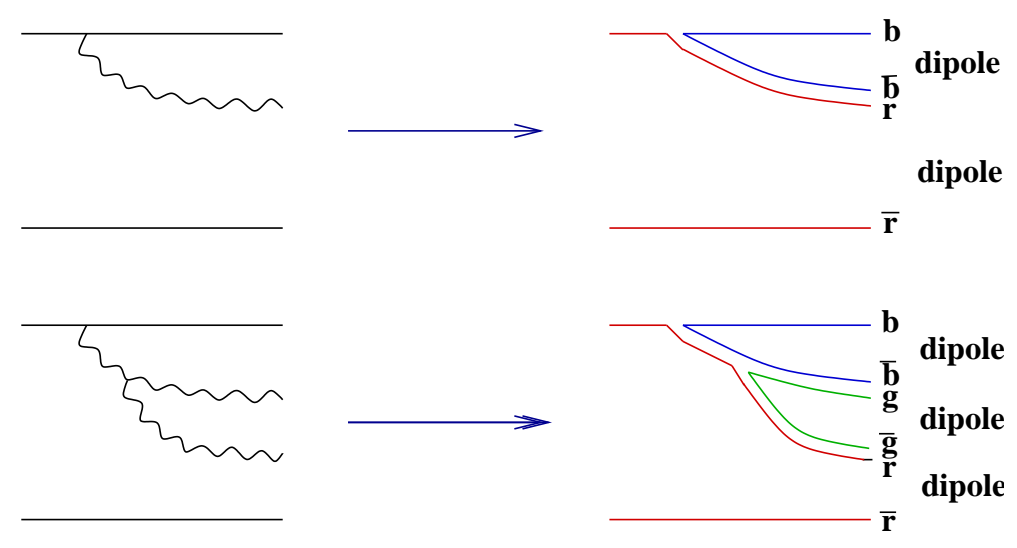

Fig. 3.5: Cascata de glúons no limite de grande $N_{c}$. A figura foi retirada da Ref.[42].

na parte superior da Fig. 3.5. A cascata de glúons relativa à evolução em rapidez é, portanto, substituída por uma cascata de dipolos (parte inferior da Fig. 3.5). Além disso, desconsiderando qualquer tipo de correlação entre os dipolos (ou seja, os dipolos interagem de forma independente com o alvo sem interagirem entre si), podemos escrever [43, 44]

$$
S_{q \bar{q} g}\left(\vec{x}_{10}, \vec{x}_{20}\right)=S_{q \bar{q}}\left(\vec{x}_{12}\right) S_{q \bar{q}}\left(\vec{x}_{20}\right)
$$

Agora, lembrando que $S=1-\mathcal{N}, \mathcal{N}_{q \bar{q} g}$ pode ser reescrito em termos das amplitudes $\mathcal{N}_{q \bar{q}}$ como

$$
\begin{aligned}
\mathcal{N}_{q \bar{q} g} & =1-S_{q \bar{q}}\left(\vec{x}_{12}\right) S_{q \bar{q}}\left(\vec{x}_{20}\right) \\
& =1-\left(1-\mathcal{N}_{q \bar{q}}\left(\vec{x}_{12}\right)\right)\left(1-\mathcal{N}_{q \bar{q}}\left(\vec{x}_{20}\right)\right) \\
& =\mathcal{N}_{q \bar{q}}\left(\vec{x}_{20}\right)+\mathcal{N}_{q \bar{q}}\left(\vec{x}_{12}\right)-\mathcal{N}_{q \bar{q}}\left(\vec{x}_{12}\right) \mathcal{N}_{q \bar{q}}\left(\vec{x}_{20}\right)
\end{aligned}
$$

Levando o resultado acima na Eq. (3.49), para $C_{F}=\left(N_{c}^{2}-1\right) /\left(2 N_{c}\right) \approx N_{c} / 2$ (limite de grande $N_{c}$ ), obtemos

$$
\frac{\partial \mathcal{N}\left(x_{10}, Y\right)}{\partial Y}=\frac{\alpha_{s} N_{c}}{2 \pi^{2}} \int \mathrm{d} x_{2} \frac{x_{10}^{2}}{x_{20}^{2} x_{21}^{2}}\left[\mathcal{N}\left(x_{12}\right)+\mathcal{N}\left(x_{20}\right)-\mathcal{N}\left(x_{10}\right)-\mathcal{N}\left(x_{12}\right) \mathcal{N}\left(x_{20}\right)\right]
$$

onde a partir de agora, omitimos o índice $q \bar{q}$ das amplitudes. A equação acima é a equação BK. Ela apresenta as seguintes características:

- Dado uma condição inicial $\mathcal{N}\left(Y=Y_{0}\right)$, a equação BK prediz a amplitude de espalhamento para um $Y>Y_{0}$.

- Para $\mathcal{N}$ pequeno (abaixo do limite de disco negro), podemos desprezar o termo não linear. Desta forma, obtemos a equação BFKL no espaço das posições. 
- Tomando $\mathcal{N}=1$ (limite de disco negro e máximo valor de $\mathcal{N}$ ), o lado direito da Eq. (3.52) se anula e, portanto, não existe mais evolução em rapidez (saturação).

- As divergências do Kernel em $x_{20}=x_{21} \rightarrow 0$ são regularizadas pela transparência de cor $\mathcal{N}\left(x_{i j}=0\right)=0$. Isso ocorre para pequenos dipolos, ou seja, $x_{i j} \lesssim 1 / Q_{s}$. Neste caso, os dipolos espalham muito pouco. Em $x_{i j}=0$, temos neutralidade de cor e o dipolo não espalha via interação forte.

- Para grandes dipolos, temos $x_{i j} \gtrsim 1 / Q_{s}$. Os dipolos espalham com probabilidade quase 1, mas nunca maior.

No apêndice B, apresentamos alguma soluções aproximadas das equações BK e BFKL, válidas em certos regimes cinemáticos.

\subsection{Modelos fenomenológicos para $\mathcal{N}$}

Na seção anterior, apresentamos a equação BK, a qual nos fornece a evolução da parte imaginária da amplitude de espalhamento frontal dipolo-alvo como função da energia e discutimos que uma de suas principais características é que ela respeita o vínculo de unitariedade. Por outro lado, soluções analíticas da BK só são conhecidas em regiões cinemáticas restritas (veja o apêndice B). Portanto, devemos recorrer a modelos fenomenológicos que contenham as principais características esperadas para $\mathcal{N}$, como o limite de transparência (ou neutralidade) de cor e a saturação. Nesta seção, vamos apresentar os modelos utilizados nessa tese para a seção de choque $\sigma_{\text {dip }}$ e a amplitude de espalhamento $\mathcal{N}$ para a interação dipolo-próton.

\subsubsection{Modelo GBW}

O modelo fenomenológico GBW (devido à Golec-Biernat e Wusthoff) é um dos modelos mais simples para $\sigma_{d i p}$. Ele é dado por $[45,46]$

$$
\sigma_{d i p}(x, r)=\sigma_{0}\left(1-e^{-r^{2} Q_{s}^{2}(x) / 4}\right)
$$

onde $\sigma_{0}$ é uma constante e $Q_{s}(x)$ é a escala de saturação, a qual é definida por

$$
Q_{s}^{2}(x)=\left(\frac{x_{0}}{x}\right)^{\lambda_{G B W}} \mathrm{GeV}^{2}
$$

Nessa tese, utilizamos utilizamos duas versões do modelo GBW: a versão proposta em $[45,46]$, a qual denotaremos por GBW, e a versão proposta em [47], a qual denotaremos por GBW KSX (devido à Kozlov, Shoshi e Xiang), que se trata de uma atualização do modelo GBW. A Tab.3.1 mostra os conjuntos de parâmetros desses dois modelos. 


\begin{tabular}{||c|c|c|c||}
\hline \hline & $\sigma_{0}(\mathrm{mb})$ & $\lambda_{G B W}$ & $x_{0}$ \\
\hline GBW & 23,03 & 0,288 & $3,04 \times 10^{-4}$ \\
\hline GBW KSX & 22,17 & 0,285 & $4,11 \times 10^{-4}$ \\
\hline \hline
\end{tabular}

Tab. 3.1: Parâmetros do modelo $G B W$.

A Eq. (3.53) traz dois comportamentos esperados para $\sigma_{\text {dip }}$ : para dipolos muito pequenos $\left(r \ll 1 / Q_{s}\right)$, temos

$$
\begin{aligned}
\sigma_{d i p} & \approx \sigma_{0}\left(1-1+\frac{r^{2} Q_{s}^{2}(x)}{4}\right) \\
& =\frac{r^{2} Q_{s}^{2} \sigma_{0}}{4} \\
& \propto r^{2},
\end{aligned}
$$

que é o comportamento de transparência de cor, ou seja, para pequenos dipolos $\sigma_{d i p}$ é proporcional ao tamanho do dipolo ao quadrado; já, para dipolos muito grandes $\left(r \gg 1 / Q_{s}^{2}\right)$, temos

$$
\sigma_{d i p} \approx \sigma_{0}
$$

isto é, $\sigma_{d i p}$ satura para um valor constante.

\subsubsection{Modelo IIM}

Iancu, Itakura e Munier, propuseram um modelo para $\sigma_{\text {dip }}$ que leva em conta duas soluções analíticas válidas para certas regiões cinemáticas da equação BK (as quais são discutidas em mais detalhes no apêndice B): a solução da BFKL próximo à região de saturação e a equação BK dentro da região de saturação (Lei de Levin-Tuchin). O modelo IIM é uma interpolação suave destas duas soluções tal que a forma de $\sigma_{\text {dip }}$ é [48]

$$
\sigma_{d i p}=\sigma_{0} \begin{cases}\mathcal{N}_{0}\left(\frac{r Q_{s}}{2}\right)^{2\left[\gamma_{s}+(1 /(\kappa \lambda Y)) \ln \left(2 / r Q_{s}\right)\right]} & , r Q_{s} \leq 2 \\ 1-e^{-A \ln ^{2}\left(B r Q_{s}\right)} & , r Q_{s}>2\end{cases}
$$

onde $Y=\ln (1 / x), \gamma_{s}=0,63, \kappa=9.9 \mathrm{e}$

$$
Q_{s}(x)=\left(\frac{x_{0}}{x}\right)^{\lambda / 2}
$$

Os parâmetros $\sigma_{0}, \mathcal{N}_{0}, \lambda$ e $x_{0}$ foram determinados por um fit dos dados de $F_{2}$ de HERA. Os coeficientes $A$ e $B$ são determinados pela condição de que $\sigma_{\text {dip }}$ e sua derivada em relação a $r Q_{s}$ sejam contínuas em $r Q_{s}=2$, de forma que

$$
\begin{aligned}
A & =-\frac{\mathcal{N}_{0}^{2} \gamma_{s}^{2}}{\left(1-\mathcal{N}_{0}\right)^{2} \ln \left(1-\mathcal{N}_{0}\right)} \\
B & =\frac{1}{2}\left(1-\mathcal{N}_{0}\right)^{-\left(1-\mathcal{N}_{0}\right) /\left(\mathcal{N}_{0} \gamma_{s}\right)}
\end{aligned}
$$


Em nossos trabalhos, utilizamos atualizações deste modelo feita na Ref. [49], o qual denotaremos por IIM-S (devido à Soyez), e da Ref.[50] que denotaremos por IIM-RS (devido à Rezaeian e Schmidt). Os parâmetro do modelo fornecidos por essas duas referências são apresentados na Tab.3.2.

\begin{tabular}{||c|c|c|c|c|c|c||}
\hline \hline & $\sigma_{0}(\mathrm{mb})$ & $\gamma_{S}$ & $x_{0}$ & $\lambda$ & $\kappa$ & $\mathcal{N}_{0}$ \\
\hline IIM-S & 26,25 & 0.6194 & $0,2131 \times 10^{-4}$ & 0,2545 & 9,9 & 0,7 \\
\hline IIM-RS & 21,85 & 0,762 & $6,226 \times 10^{-5}$ & 0,2319 & 9,9 & 0,7 \\
\hline \hline
\end{tabular}

Tab. 3.2: Parâmetros do modelo IIM.

\subsubsection{Modelo bCGC}

Uma modificação do modelo acima, a qual leva em conta uma dependência de $\sigma_{\text {dip }}$ no parâmetro de impacto, é o chamado modelo bCGC (CGC dependente do parâmetro de impacto). Nele a seção de choque de dipolo é escrita como [31]

$$
\frac{\mathrm{d} \sigma_{d i p}}{\mathrm{~d}^{2} b}=2 \mathcal{N}(x, r, b)=2 \times \begin{cases}\mathcal{N}_{0}\left(\frac{r Q_{s}}{2}\right)^{2\left[\gamma_{s}+(1 /(\kappa \lambda Y)) \ln \left(2 / r Q_{s}\right)\right]} & , r Q_{s} \leq 2 \\ 1-e^{-A \ln ^{2}\left(B r Q_{s}\right)} & , r Q_{s}>2\end{cases}
$$

onde $Q_{s}(b)$ possui a seguinte dependência em $b$

$$
Q_{s} \equiv Q_{s}(x, b)=\left(\frac{x_{0}}{x}\right)^{\lambda / 2}\left[\exp \left(-\frac{b^{2}}{2 B_{C G C}}\right)\right]^{1 /\left(2 \gamma_{s}\right)} .
$$

Além do modelo bCGC, utilizamos também sua atualização presente na Ref.[50], o qual denotaremos por bCGC NEW. Os parâmetros para esse modelo presentes nas Refs.[31, 50] são apresentados na Tab.3.3.

\begin{tabular}{||c|c|c|c|c|c|c||}
\hline \hline & $B_{C G C}\left(\mathrm{GeV}^{2}\right)$ & $\gamma_{S}$ & $x_{0}$ & $\lambda$ & $\kappa$ & $\mathcal{N}_{0}$ \\
\hline bCGC & 7,5 & 0.46 & $1,84 \times 10^{-6}$ & 0,119 & 9,9 & 0,558 \\
\hline bCGC NEW & 5,5 & 0,6599 & 0,00105 & 0,2063 & 9,9 & 0,3358 \\
\hline \hline
\end{tabular}

Tab. 3.3: Parâmetros do modelo bCGC.

\subsubsection{Modelo rcBK}

Acima citamos três modelos fenomenológicos para a seção de choque de dipolo dentro de várias possibilidades que podem ser encontradas na literatura. Outro modelo usado 
nesta tese é o chamado rcBK ("running coupling" BK) no qual a solução da equação BK é calculada numericamente [51]. Ele leva em conta correções em next-to-leading order (NLO) e considera a constante de acoplamento $\alpha_{s}$ variável.

Na Ref. [51] foi fornecido um código em FORTRAN que calcula a seção de choque de dipolo com o modelo rcBK. Para tal modelo, devemos fornecer a condição inicial, normalmente extraída de algum modelo fenomenológico. Aqui, seguimos a Ref. [51] e usamos o modelo GBW como condição inicial.

Este modelo é bastante interessante por considerar a solução numérica da equação BK e, por isso, é considerado como o estado da arte para o cálculo de $\mathcal{N}$. Porém, esta solução é válida apenas para $x<0,01$. Como será discutido na subseção 5.2.2, esta limitação em $x$ leva a uma limitação cinemática em nossos resultados para a distribuição de rapidez. Portanto, os modelos fenomenológicos citados anteriormente são fundamentais para a apresentação de predições para todo a extensão de rapidez.

\subsection{Evidências experimentais da saturação}

Nesta seção vamos apresentar algumas evidências experimentais da saturação. Como veremos, existe uma série de resultados relacionados a observáveis que foram estudados no HERA, no RHIC e no LHC que possuem uma boa concordância com o formalismo do CGC. Este fato é extremamente interessante e nos mostra que o formalismo do CGC nos leva a uma descrição universal e auto-consistente de tais resultados.

Vamos começar apresentando dois resultados do HERA que podem ser explicados pela física de saturação. Uma das predições que podem ser feitas com o CGC é o chamado scaling geométrico (Fig. 3.6 à esquerda). Em pequeno $x$, as seções de choque $\gamma^{*} p$ dependem apenas da seguinte variável

$$
\tau=\frac{Q^{2}}{Q_{s}^{2}(x)} \sim \frac{1}{r^{2} Q_{s}^{2}(x)} .
$$

Tal comportamento é refletido explicitamente nos modelos fenomenológicos de dipolo. O primeiro gráfico da Fig. 3.6 mostra este comportamento. No segundo gráfico da Fig. 3.6, apresentamos resultados experimentais para a razão entre as seções de choque difrativa e total. A física de saturação prediz que esta razão deva ser aproximadamente constante com a energia [46]. Tal predição é verificada pela figura.

Vamos agora apresentar alguns dados dos colisores hadrônicos RHIC e LHC e mostrar como predições feitas com o CGC possuem boa concordância com os dados. Segundo a física de saturação, a multiplicidade de hádrons produzidos em colisões $p p$ e $A A$ (denotada por $\mathrm{d} N / \mathrm{d} \eta$, sendo $\eta$ a pseudo-rapidez) por unidade de área trasversa deve ser proporcional à escala de saturação

$$
\frac{1}{S_{\perp}} \frac{\mathrm{d} N}{\mathrm{~d} \eta} \propto Q_{s}^{2}(x)
$$

A equação acima está associada com o fato de que, em altas energias, a única escala relevante para o processo de espalhamento é a escala de saturação $Q_{s}^{2}$. Além disso, como $Q_{s}^{2} \sim x^{-\lambda}$, 

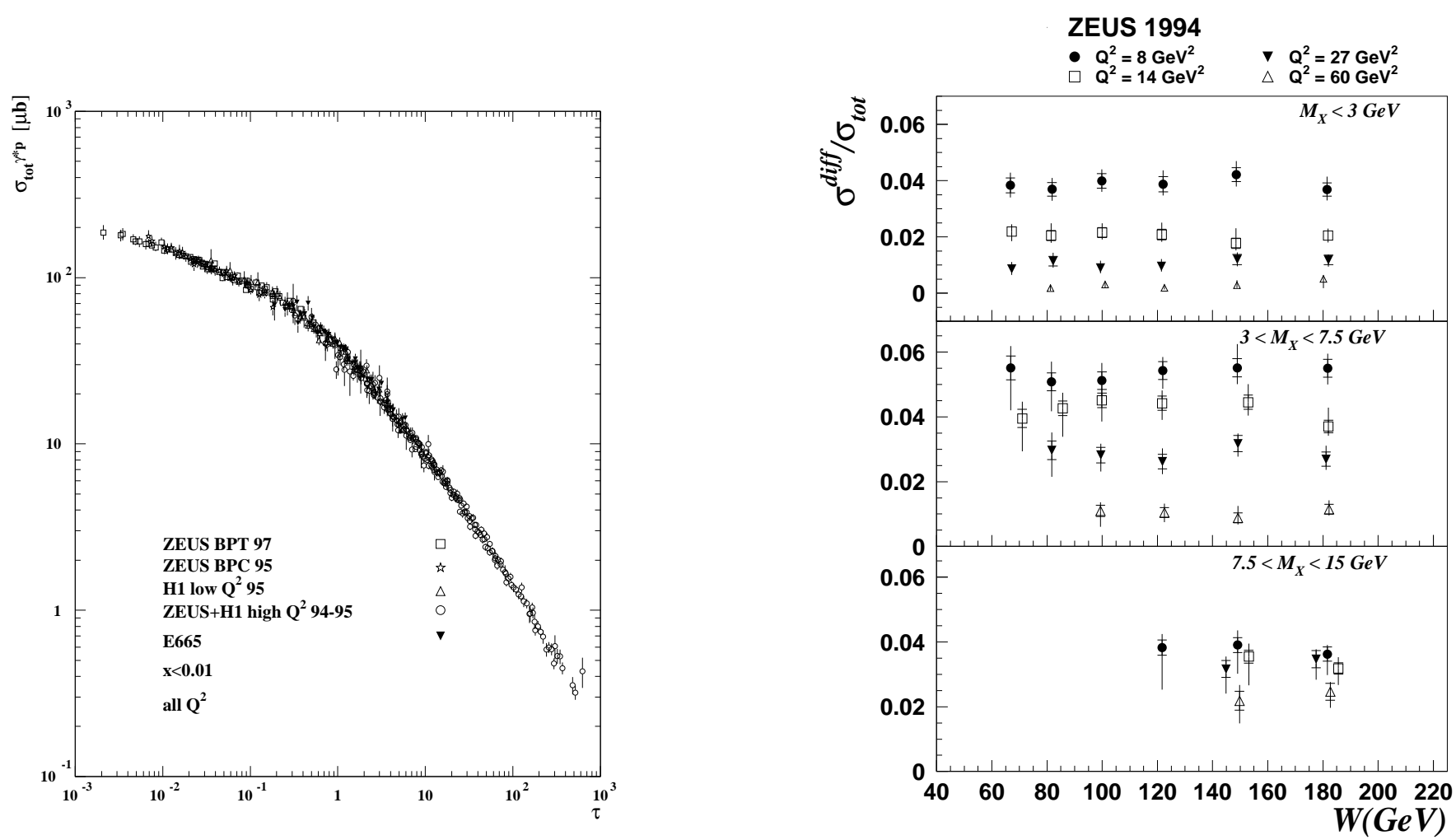

Fig. 3.6: Scaling geométrico observado na seção de choque $\sigma^{\gamma^{*} p}$ [52] (à esquerda) e Razão $\sigma_{\text {diff }} / \sigma_{\text {tot }}[53]$ (à direita).

temos que a multiplicidade deve crescer com a energia. O primeiro gráfico da Fig. 3.7 mostra que a física de saturação possui uma concordância excelente com os dados experimentais para a mutiplicidade como função da pseudo-rapidez em colisões $A A$ para dados do RHIC. No segundo gráfico da Fig. 3.7, são apresentadas as multiplicidades de hádrons produzidos em colisões $p p$ e $A A$ como função da energia para dados do RHIC e do LHC. Novamente a física de saturação leva a uma boa concordância com os dados.

Por fim, outra predição feita pela física de saturação é a de que o momento transversal médio $\left\langle p_{T}\right\rangle$ das partículas produzidas deva ser proporcional à escala de saturação e, portanto, deve crescer com a energia. Novamente, esta é uma manifestação do fato de que em altas energias, esta é a única escala relevante do problema. A Fig. 3.8 mostra dados experimentais da colaboração CMS para o comportamento de $\left\langle p_{T}\right\rangle$ contra $\sqrt{s}$ que parecem demonstrar este comportamento.

Nesta seção, mostramos algumas evidências experimentais da saturação. Em particular, destacamos que este formalismo é capaz de descrever um grande conjunto de dados rela- 

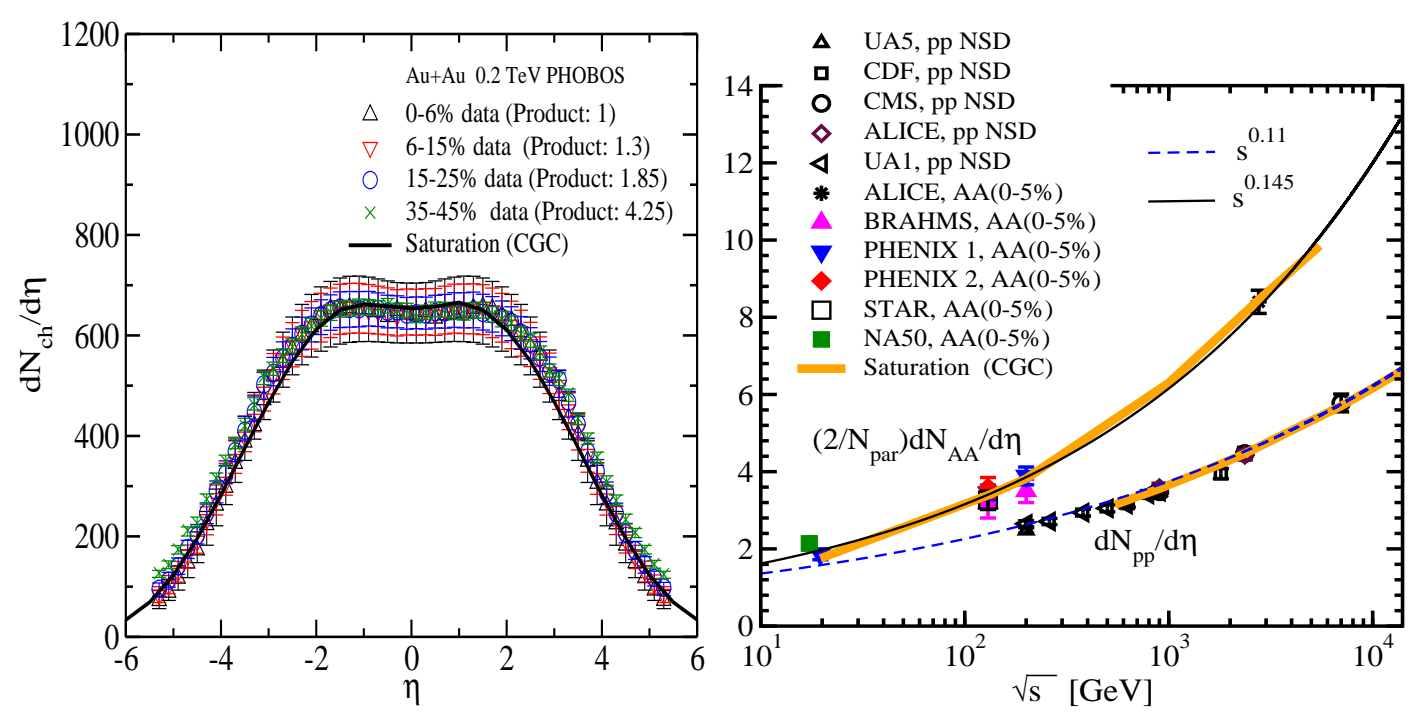

Fig. 3.7: Dependência da multiplicidade hadrônica com a rapidez em colisões AA no RHIC (à esquerda) e dependência da multiplicidade hadrônica com a energia em colisões pp e AA para energias do RHIC e do LHC (à direita). A figura foi retirada da Ref.[54].

cionados a diferentes experimentos. Aqui, mostramos apenas alguns resultados. Maiores detalhes do que foi discutido e um número maior de evidências da saturação podem ser encontrados nas Refs. [9, 37]. Por fim, cabe salientar que embora a física de saturação seja bem-sucedida na explicação de diversos processos, uma resposta concreta sobre a existência da saturação se torna difícil, tendo em vista que outras aproximações são capazes de explicar vários desses efeitos sem utilizar o fenômeno de saturação.

\subsection{Conclusão}

Neste capítulo introduzimos as principais equações de evolução da QCD. Inicialmente apresentamos a equação BFKL, que é uma equação linear, e derivamos algumas de suas soluções aproximadas. Derivamos o limite de Froissart e mostramos que as soluções das equações DGLAP e BFKL violam tal limite. A seguir apresentamos a equação GLR, a qual é uma equação de evolução não linear. Aqui, definimos o conceito de escala de saturação e justificamos o fenômeno da saturação de glúons. No final do capítulo apresentamos um caminho para a derivação da equação BK, que também é uma equação não linear. De forma geral, discutimos conceitos importantes para o estudo da QCD em altas energias, mostrando os problemas das equações lineares da QCD no limite de pequeno $x$ e apresentando o 


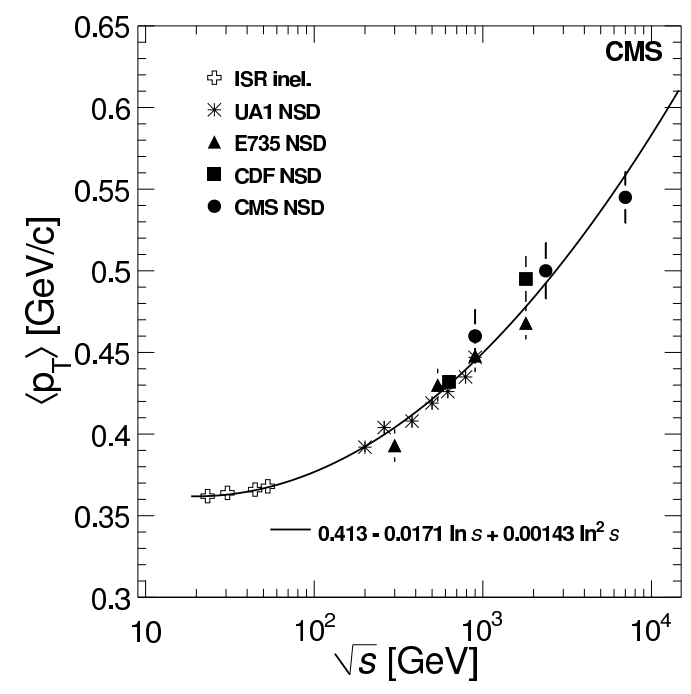

Fig. 3.8: Dependência de $\left\langle p_{T}\right\rangle$ com $\sqrt{s}$. A figura foi retirada da Ref.[55].

fenômeno de saturação e algumas de suas evidências experimentais. Em particular, com o formalismo de dipolo introduzido nesse capítulo para o caso inclusivo (o qual será estendido no cap.5 para o caso exclusivo), temos condições de estudar interações $\gamma$-alvo. No próximo capítulo, vamos discutir como podemos estudar o espalhamento entre dois hádrons em uma colisão ultraperiférica uma vez conhecida a seção de choque de interação $\gamma$ - alvo (onde, em nossos casos de interesse, o alvo pode ser um hádron ou outro fóton). Para isso, vamos estudar o chamado método de fótons equivalentes. Vamos também argumentar que esse método pode ser utilizado para estudar a produção exclusiva de mésons vetoriais, que é o tema central dessa tese e é uma forma alternativa ao DIS para o estudo da física de altas energias. O estudo fenomenológico desses tipos de processos se mostram bastante interessantes na atualidade, tendo em vista que o LHC fornece uma oportunidade única (isto é, em energias nunca antes alcançadas por outro colisor) para o estudo dos mesmos. 


\section{Capítulo 4}

\section{Processos foto-induzidos em colisões hadrônicas}

Neste trabalho, estudamos processos foto-induzidos (induzidos por fotons) através da aproximação de fótons equivalentes. A aproximação de fótons equivalentes é um método para o cálculo de seções de choque para vários processos eletromagnéticos. Enrico Fermi foi um dos primeiros físicos a pensar neste método [56]. Em seu trabalho, Fermi tratou o campo eletromagnético de uma carga elétrica em movimento como um fluxo de fótons virtuais. Algum tempo depois, Wezsäcker e Williams (Refs.[57, 58]) estenderam essa aproximação para partículas ultra-relativísticas.

Analisando o campo eletromagnético de uma carga elétrica em movimento, observamos que o aumento de sua velocidade faz com que as linhas campo eletromagnético se agrupem cada vez mais na direção transversal ao seu movimento (Fig. 4.1). Este campo a uma dada distância da trajetória da partícula é semelhante ao campo de um fóton real. A idéia do método é substituir o efeito do campo eletromagnético gerado pela carga elétrica em movimento (com respeito a um dado referencial inercial) por um fluxo de fótons quase reais associado a ela.

Em uma colisão hádron-hádron ultraperiférica, ou seja, tal que o parâmetro de impacto é maior do que a soma de seus raios $\left(b>R_{1}+R_{2}\right)$, os hádrons atuam como fontes de fótons podendo ocorrer colisões fóton-núcleo, ou fóton-fóton. A exigência de que o parâmetro de impacto seja grande, implica que interações hadrônicas sejam suprimidas. Portanto, numa colisão ultraperiférica a interação inicial é descrita pela QED e a interação fóton-hádron ou fóton-fóton, que ocorre posteriormente, pode envolver interações descritas pela QCD.

O interesse atual em colisões ultraperiféricas se deve ao fato de existirem várias oportunidades de estudo experimental oferecidas pelo LHC [59]. Em particular, em colisões envolvendo $\mathrm{Pb}(Z=82)$ no LHC, as seções de choque são amplificadas por causa dos campos extremamente intensos gerados pela carga do íon. Além disso, com o LHC é possível explorar a física de pequeno $x$ em colisões $\gamma p$ e $\gamma A$ em $W \gtrsim 1 \mathrm{TeV}$ (onde $W$ é a energia de centro de massa fóton-hádron). Com estas energias é possível ter acesso a valores de $x$ que são uma ordem de grandeza menores do que os alcançados no HERA [60]. Isto significa que o LHC está em um regime cinemático onde possíveis efeitos da física não linear são muitas vezes maiores do que em qualquer outro acelerador já construído.

Para o caso de um núcleo atuando como fonte de fótons, temos que as seções de choque serão realçadas por um fator $Z^{2}$ se os fótons forem emitidos por todo o núcleo de forma 

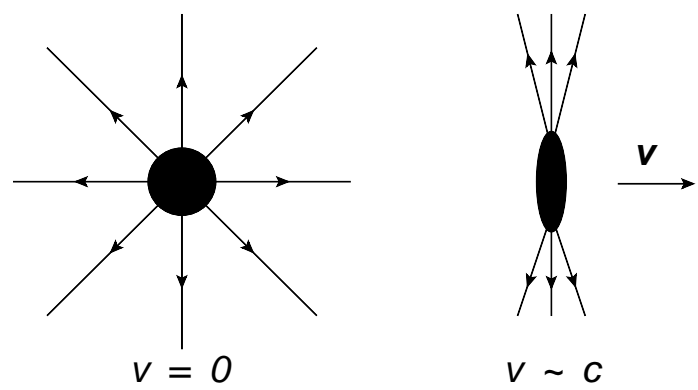

Fig. 4.1: Campo eletromagnético de uma carga para $v=0$ (à esquerda) e $v \sim c$ (à direita).

coerente. Isso implica que o comprimento de onda do fóton deve ser da ordem do raio do núcleo, isto é $\lambda \sim R_{A}$. Dessa forma, a virtualidade do fóton é limitada por

$$
Q^{2} \leq \frac{1}{R_{A}^{2}} \rightarrow Q_{\max }^{2}=\frac{1}{R_{A}^{2}}
$$

Como $Q^{2}=-k^{2}$, conforme discutido em detalhes no apêndice $\mathrm{C}$, temos (para $k=\left(\omega, k_{x}=\right.$ $\left.\omega / v, \vec{k}_{\perp}\right)$, onde o eixo $x$ é o eixo onde ocorre a colisão)

$$
Q^{2} \approx \frac{\omega^{2}}{\gamma^{2}}+\vec{k}_{\perp}^{2}
$$

Usando a equação acima com a Eq.4.1, obtemos para a energia e momentum máximos dos fótons

$$
\omega_{\max } \approx \frac{\gamma}{R_{A}}
$$

$\mathrm{e}$

$$
\left|\vec{k}_{\perp, \max }\right| \approx \frac{1}{R_{A}}
$$

respectivamente.

Na Tab.4.1 listamos alguns parâmetros e limites cinemáticos do RHIC e do LHC pertinentes em colisões $\gamma$-alvo e $\gamma-\gamma$. Nela são apresentados os valores para a luminosidade em diferentes configurações $\left(\mathcal{L}_{A B}\right)$, energia de centro de massa núcleon-núcleon $\left(\sqrt{s_{N N}}\right)$, fatores de Lorentz $\left(\gamma_{L}\right)$, energias de centro de massa $\gamma p(A)$ máximas $\left(\sqrt{s_{\gamma N}}=W_{\gamma N}=\right.$ $\left.\left[2 \omega_{\max } \sqrt{s_{N N}}\right]^{1 / 2}\right)$, energias máximas do fóton $\left(\omega_{\max }=\gamma_{L} / R_{A}\right)$ e energias do centro de massa $\gamma \gamma$ máximas $\left(\sqrt{s_{\gamma \gamma}}=W_{\gamma \gamma}=\sqrt{4 \omega_{1, \max } \omega_{2, \max }}\right)$. Uma breve comparação entre a cinemática 


\begin{tabular}{|c|c|c|c|c|c|c|c|}
\hline$A B$ & $\begin{array}{c}\mathcal{L}_{A B} \\
\left(\mathrm{mb}^{-1} \mathrm{~s}^{-1}\right)\end{array}$ & $\begin{array}{c}\sqrt{s_{N N}} \\
(\mathrm{TeV})\end{array}$ & $\begin{array}{c}E_{\text {beam }} \\
(\mathrm{TeV})\end{array}$ & $\gamma_{L}$ & $\begin{array}{c}\omega_{\max } \\
(\mathrm{GeV})\end{array}$ & $\begin{array}{c}\sqrt{s_{\gamma N}^{\max }} \\
(\mathrm{GeV})\end{array}$ & $\begin{array}{c}\sqrt{s_{\gamma \gamma}^{\max }} \\
(\mathrm{GeV})\end{array}$ \\
\hline \multicolumn{7}{|c|}{ RHIC } \\
\hline $\mathrm{Au}+\mathrm{Au}$ & 0.4 & 0.2 & 0.1 & 106 & 3.0 & 34.7 & 6.0 \\
\hline$p$ & 6000 & 0.5 & 0.25 & 266 & 87 & 296 & 174 \\
\hline $\mathrm{O}+\mathrm{O}$ & 160 & 7 & 3.5 & 3730 & 243 & 1850 & 486 \\
$\mathrm{Ar}+\mathrm{Ar}$ & 43 & 6.3 & 3.15 & 3360 & 161 & 1430 & 322 \\
$\mathrm{~Pb}+\mathrm{Pb}$ & 0.42 & 5.5 & 2.75 & 2930 & 81 & 950 & 162 \\
\hline$p \mathrm{O}$ & 10000 & 9.9 & 4.95 & 5270 & 343 & 2610 & 686 \\
$p \mathrm{Ar}$ & 5800 & 9.39 & 4.7 & 5000 & 240 & 2130 & 480 \\
$p \mathrm{~Pb}$ & 420 & 8.8 & 4.4 & 4690 & 130 & 1500 & 260 \\
\hline$p p$ & $10^{7}$ & 14 & 7 & 7455 & 2452 & 8390 & 4904 \\
\hline
\end{tabular}

Tab. 4.1: Parâmetros e limites cinemáticos para processos fóton-hádron e fóton-fóton no RHIC e no LHC [60].

dos dois colisores mostra que o LHC nos permite o estudo em colisões de altíssimas energias relativamente ao RHIC.

Um atrativo do estudo experimental de colisões ultraperiféricas, é que elas são processos limpos, especialmente quando comparados com processos puramente hadrônicos, tendo em vista que um ou ambos os hádrons do estado inicial não se fragmentam. Isso se deve ao fato de que o fóton não porta carga e, dessa forma, não emite partículas. Por conta disso, o fóton gera um gap de rapidez (isto é, regiões do espaço de fase onde não existem partículas) que separa o estado produzido das partículas presentes no estado inicial. Esta característica favorece o estudo da produção exclusiva de partículas em colisões hadrônicas, que é o nosso objeto de estudo. Outra vantagem é que o LHC é uma fábrica de mésons vetoriais devido às altas taxas de produção destas partículas [61].

Nas seções seguintes, mostraremos as principais características da chamada aproximação de fótons equivalntes. Uma derivação das fórmulas principais é apresentada no apêndice C. Logo após, seção faremos uma breve descrição de como a produção exclusiva de mésons vetoriais vem sendo estudada desde o HERA até o LHC. Por fim, apresentamos alguns dados experimentais do LHC referentes à produção de partículas em processos foto-induzidos.

\subsection{O método de fótons equivalentes}

Seja um processo de espalhamento entre duas partículas carregadas $A$ e $B$ em energias ultra-relativísticas. Neste processo, consideramos que a partícula $A$ emite um fóton virtual $\gamma^{*}$ que interage com a partícula $B$ produzindo um sistema de partículas $X$ no estado final. 
Tal processo pode ser denotado pela seguinte equação

$$
A+B \rightarrow A+\left(\gamma^{*}+B\right) \rightarrow A+X .
$$

Consideremos agora um processo de espalhamento onde um fóton real $\gamma$ interage com a mesma partícula $B$ e produz o mesmo estado final $X$, expresso por

$$
\gamma+B \rightarrow X
$$

Se no primeiro processo, o fóton virtual tiver uma virtualidade muito baixa, isto é, se $\gamma^{*}$ for um fóton quase real, a Eq.(4.5) pode ser reescrita como

$$
A+B \rightarrow A+(\gamma+B) \rightarrow A+X .
$$

Isso significa que o processo entre parênteses na equação acima pode ser aproximado pelo processo da Eq.(4.6).

Neste caso, é possível mostrar que a seção de choque do processo $A+B$ pode ser escrita em termos da seção de choque do processo $\gamma+B$ como (conforme apêndice C e Ref.[62])

$$
\sigma_{A+B \rightarrow A+X}=\int \frac{\mathrm{d} \omega}{\omega} n_{A}(\omega) \sigma_{\gamma+B \rightarrow X}(\omega)
$$

onde, na equação acima, as seções de choque $\sigma_{A+B \rightarrow A+X}$ e $\sigma_{\gamma+B \rightarrow X}$ são respectivamente referentes aos processos das Eq.(4.7) e Eq.(4.6). Além disso, $\omega$ é a energia do fóton e $n_{A}(\omega)$ é o chamado espectro de fótons equivalentes emitidos pela partícula $A$ (que depende apenas da partícula $A$ ). Portanto, em altas energias e baixas virtualidades, a seção de choque do processo $A+B \rightarrow A+X$ pode ser fatorizada em termos da seção de choque do processo $\gamma+B \rightarrow X$ e do espectro de fótons equivalentes da partícula $A$.

A quantidade $n(\omega)$ pode ser calculada pela QED para o caso em que a fonte de fótons $A$ é uma partícula elementar carregada (um lépton, por exemplo), fornecendo [62]

$$
\begin{aligned}
n(\omega) & =4 \alpha \int \frac{\left|\vec{k}_{\perp}\right|^{2} \mathrm{~d}^{2}\left|\vec{k}_{\perp}\right|}{(2 \pi)^{2}} \frac{1}{\left(\vec{k}_{\perp}^{2}+\frac{\omega^{2}}{\gamma^{2}}\right)^{2}} \\
& \approx \frac{2}{\pi} \alpha \log \left(\frac{\gamma m_{B}}{\omega}\right),
\end{aligned}
$$

onde $\gamma$ é o fator de Lorentz e $m_{B}$ é a massa da partícula $B$. O caso de partículas com estrutura interna atuando como fonte de fótons será discutido na próxima seção. A equação (4.8) é uma das ferramentas básicas desta tese. Ela nos permite estudar a produção de partículas em colisões $p p, p P b$ e $P b P b$ no LHC através do conhecimento de seções de choque de interação $\gamma p(P b)$. Tais seções de choque são calculadas com o auxílio do formalismo de dipolo de cor, o qual foi desenvolvido no capítulo anterior para o caso da produção inclusiva. Para os processos que são de interesse no presente trabalho, as seções de choque $\gamma p(P b)$ serão discutidas nos próximos capítulos.

A Eq.(4.8) pode ser generalizada para o caso onde ocorrem interações $\gamma \gamma$. Este tratamento é interessante para o caso em que conhecemos a seção de choque de interação entre dois fótons $\sigma_{\gamma \gamma}$ dando origem a um estado final $X$. Esta generalização leva a

$$
\sigma_{A B}=\iint \mathrm{d} \omega_{1} \mathrm{~d} \omega_{2} \frac{n_{A}\left(\omega_{1}\right)}{\omega_{1}} \frac{n_{B}\left(\omega_{2}\right)}{\omega_{2}} \sigma_{\gamma \gamma}\left(\omega_{1}, \omega_{2}\right)
$$

e nos permite estudar processos $\gamma \gamma$ em colisões hadrônicas. 


\subsection{Colisões ultraperiféricas em colisores hadrônicos}

Nessa seção vamos discutir como os resultados acima podem ser generalizados para o caso onde prótons e núcleos atuam como fontes de fótons. Isso é extremamente interessante pois possibilita o estudo deste tipo de colisão no LHC. Além disso, este tema está fortemente relacionado aos temas estudados na presente tese.

\subsubsection{Fluxo de fótons independente do parâmetro de impacto}

Como comentado na seção anterior, a aproximação de fótons equivalentes pode ser derivada pela QED, para o caso onde a fonte de fótons é uma partícula elementar. Portanto, a Eq.(4.9) considera que os fótons são emitidos por partículas sem estrutura interna. Entretanto, nos cálculos dessa tese, estamos interessados em colisões hadrônicas. Para o caso de partículas com estrutura interna, temos $[63,64]$

$$
f(x)=\frac{\alpha Z^{2}}{2 \pi} \frac{\left[1+(1-x)^{2}\right]}{x} \int_{Q_{\min }^{2}}^{\infty} \frac{Q^{2}-Q_{\min }^{2}}{Q^{4}}\left|F\left(Q^{2}\right)\right|^{2} \mathrm{~d} Q^{2}
$$

com

$$
x f(x)=n(\omega) ; x=\frac{\omega}{E}
$$

onde $\omega$ é a energia do fóton, $E$ e $F\left(Q^{2}\right)$ são respectivamente a energia e o fator de forma da partícula que atua como fonte de fótons (projétil) e $x$ é a fração da energia do hádron projétil portada por um dado fóton. Além disso, $Q^{2}$ é o quadrimomento transferido do projétil de massa $M_{A}$ e

$$
Q_{\min }^{2}=\left(x M_{A}\right)^{2} /(1-x)
$$

O espectro de fótons equivalentes gerados por prótons de altas energias pode ser obtido utilizando um fator de forma do tipo dipolo elétrico

$$
F\left(Q^{2}\right)=\frac{1}{\left(1+Q^{2} / 0,71 \mathrm{GeV}^{2}\right)^{2}},
$$

e é dado por [64]

$$
f(x)=\frac{\alpha}{2 \pi} \frac{\left[1+(1-x)^{2}\right]}{x}\left[\ln (\Omega)-\frac{11}{6}+\frac{3}{\Omega}-\frac{3}{2 \Omega}+\frac{1}{3 \Omega^{3}}\right]
$$

com $\Omega=1+\left(0,71 \mathrm{GeV}^{2}\right) / Q_{\text {min }}^{2}$. Este é um fluxo de fótons (obtido por Dress e Zeppenfeld [65]) amplamente utilizado na literatura.

No caso de uma partícula puntual (isto é, $F(q)=1$ ), temos $[64,66]$

$$
f(x)=\frac{\alpha Z^{2}}{\pi x}\left[2 Y K_{0}(Y) K_{1}(Y)-Y^{2}\left(K_{1}^{2}(Y)-K_{0}^{2}(Y)\right)\right]
$$




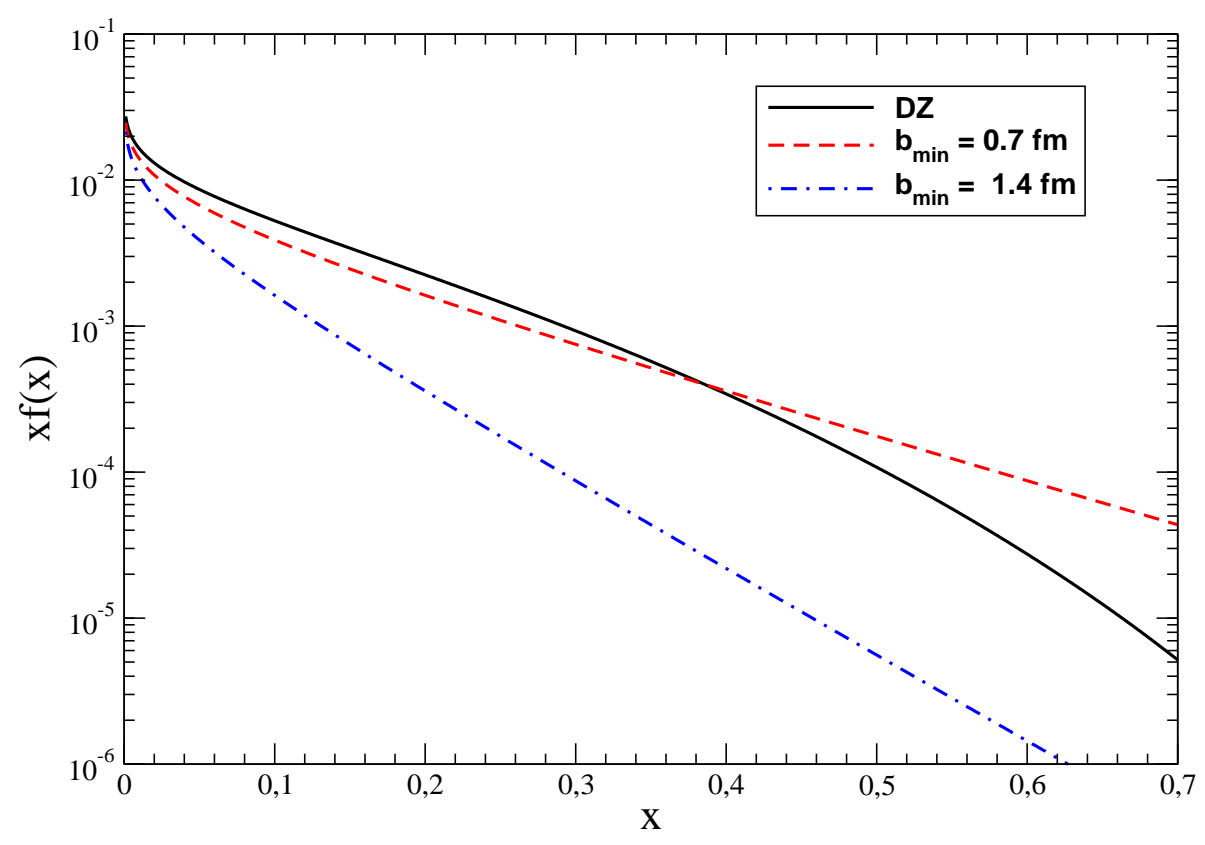

Fig. 4.2: Fluxo de fótons equivalentes associados ao próton.

onde $K_{0}$ e $K_{1}$ são funções de Bessel, $Y=x M_{A} b_{\min }$ e $b_{\min }$ é o parâmetro de impacto mínimo. Em nossos cáculos, utilizamos esse fluxo para núcleos.

A Fig. 4.2 mostra uma comparação entre os dois fluxos de fótons. Para o fluxo da Eq. (4.16), utilizamos $b_{\text {min }}=0.7 \mathrm{fm} \mathrm{e} 1.4 \mathrm{fm}$ (para $R_{p} \approx 0.7 \mathrm{fm}$ ), para mostrar o efeito da escolha do corte em parâmetro de impacto.

\subsubsection{Fluxo de fótons dependente do parâmetro de impacto}

Existem casos onde é necessário conhecer a dependência do espectro de fótons equivalentes com o parâmetro de impacto, a fim de excluir interações hadrônicas. Para exemplificar, consideremos o caso onde temos uma interação $\gamma \gamma$ em uma colisão núcleo-núcleo, que é esquematizada na Fig.4.3. Nesse caso os campos das duas partículas interagem entre si à distâncias $\vec{b}_{1}$ e $\vec{b}_{2}$ respectivas as partículas 1 e 2 (ver Fig.4.3). Por outro lado, estamos interessados apenas em colisões tais que o parâmetro de impacto da colisão seja maior do que a soma dos raios dos núcleos envolvidos. Para que isso possa ser feito, é necessário atribuírmos uma dependência das seções de choque nas distâncias $\vec{b}_{1}$ e $\vec{b}_{2}$ e consequentemente no parâmetro de impacto. Para isso, assumimos um fluxo de fótons equivalentes dependente 


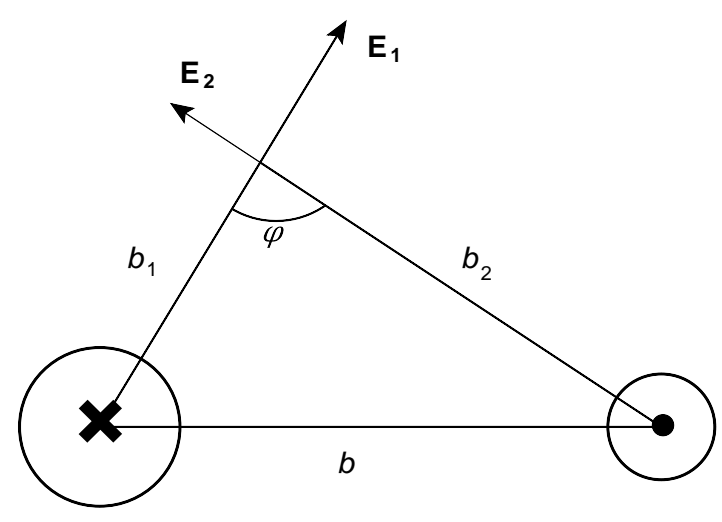

Fig. 4.3: Processo $\gamma \gamma$ em interações hadrônicas.

de $\vec{b}$, denotado por $N(\omega, \vec{b})$, o qual pode ser definido por

$$
\frac{n(\omega)}{\omega}=\int \mathrm{d}^{2} b N(\omega, b)
$$

Desta forma, a seção de choque deste processo pode ser escrita como

$$
\sigma_{A_{1} A_{2} \rightarrow A_{1} A_{2} X}(\sqrt{s})=\int \mathrm{d}^{2} b_{1} \mathrm{~d}^{2} b_{2} \mathrm{~d} \omega_{1} \mathrm{~d} \omega_{2} N_{A_{1}}\left(\omega_{1}, b_{1}\right) N_{A_{2}}\left(\omega_{2}, b_{2}\right) \sigma_{\gamma \gamma \rightarrow X}\left(\omega_{1}, \omega_{2}\right)
$$

Como uma primeira tentativa de impor a condição $b>R_{1}+R_{2}$, poderíamos tomar $b_{i, \min }=R_{i}$ ou, até mesmo $b_{i, \min }=R_{1}+R_{2}$. Contudo, isso não impediria que ocorressem colisões em parâmetros de impacto menores do que a soma dos raios dos núcleos, já que estas imposições nas distâncias $b_{1,2}$ não acarretam diretamente em um parâmetro de impacto maior do que a soma dos raios dos projéteis. A forma adequada de remover colisões em $b^{\prime} \mathrm{s}$ menores do que a soma dos raios dos núcleos é inserindo um fator de absorção (denotado por $\left.S_{a b s}^{2}(\vec{b})\right)$ dependente de $b$ no integrando da forma

$$
S_{a b s}^{2}(\vec{b})=\Theta\left[|\vec{b}|-\left(R_{1}+R_{2}\right)\right]=\Theta\left[\left|\vec{b}_{1}-\vec{b}_{2}\right|-\left(R_{1}+R_{2}\right)\right],
$$

onde agora os limites inferiores de integração são tomados como zero, caso o fluxo dependente de $b$ escolhido permita esta escolha. Caso contrário tomamos $b_{i, \min }=R_{A}$. 


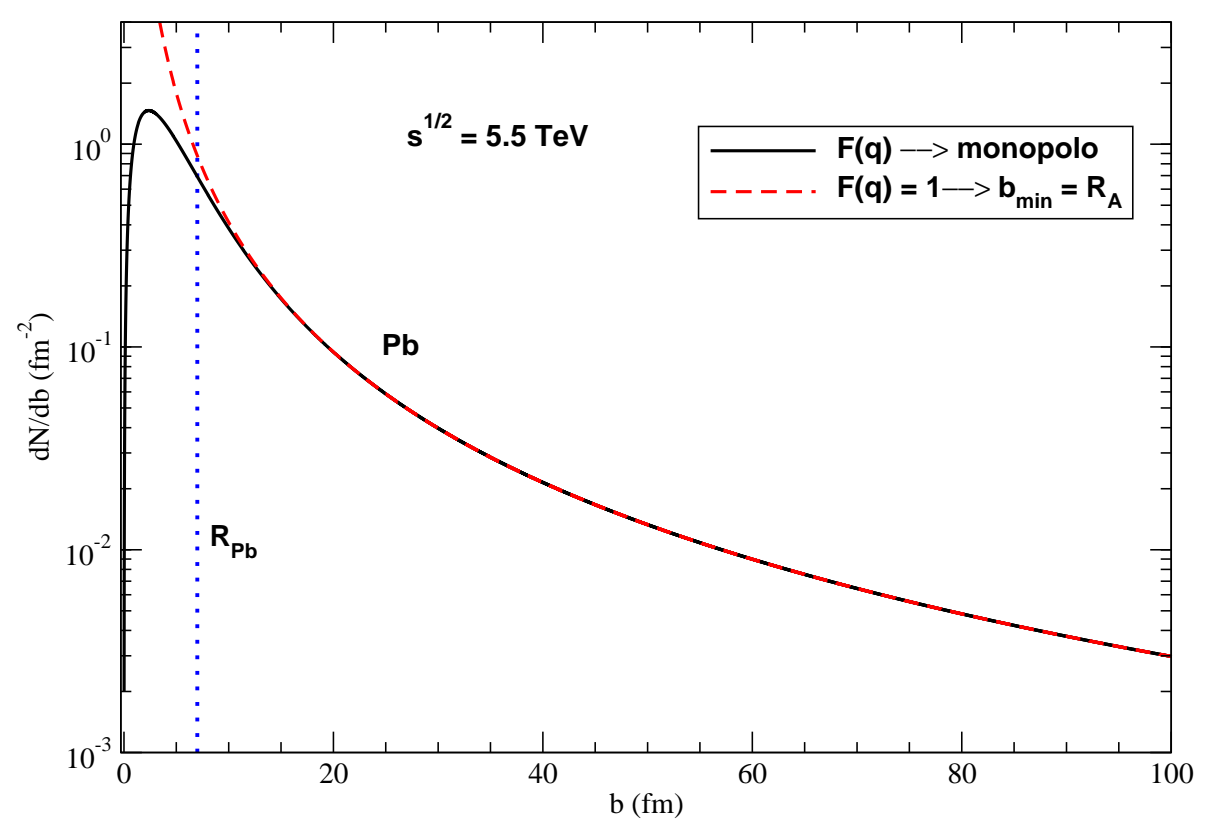

Fig. 4.4: Comparação entre os fluxos de fótons dependentes de b.

O fluxo de fótons equivalentes dependente de $b$ é dado por (veja o apêndice C)

$$
N(\omega, b)=\frac{Z^{2} \alpha}{\pi^{2} \omega b^{2}}\left|\int_{0}^{\infty} \mathrm{d} u u^{2} J_{1}(u) \frac{F\left[\frac{u^{2}+\left(\frac{b \omega}{\gamma}\right)^{2}}{b^{2}}\right]}{\left[u^{2}+\left(\frac{b \omega}{\gamma}\right)^{2}\right]}\right|^{2} .
$$

Na equação acima, $F\left(Q^{2}\right)$ é o fator de forma da partícula que atua como fonte de fótons. A seguir, vamos apresentar os fluxos de fótons para alguns dos fatores de forma utilizados em nossos trabalhos.

Para núcleos, utilizamos dois fatores de forma. No primeiro deles, tomamos $F\left(Q^{2}\right)=1$. Neste caso o fluxo é dado por

$$
N(\omega, b)=\frac{Z^{2} \alpha \xi^{2}}{\pi^{2} \omega b^{2}} K_{1}^{2}(\xi), \quad \xi=\frac{\omega b}{\gamma} .
$$

Este fluxo diverge para $b \rightarrow 0$. Portanto, devemos tomar um corte para o limite inferior nas integrais em $b$. Em geral, é escolhido $b_{\min }=R_{A}[66,67,68]$.

Outra escolha possível (e mais realista que a primeira), é um fator de forma do tipo monopolo [69], dado por

$$
F(q)=\frac{\Lambda^{2}}{\Lambda^{2}+q^{2}} ; \Lambda=0,088 \mathrm{GeV}
$$


que leva a um fluxo de fótons equivalentes dado por

$$
N(\omega, b)=\frac{Z^{2} \alpha_{e m}}{\pi^{2} \omega}\left[\frac{\omega}{\gamma} K_{1}\left(\frac{b \omega}{\gamma}\right)-\sqrt{\left(\frac{\omega^{2}}{\gamma^{2}}+\Lambda^{2}\right)} K_{1}\left(b \sqrt{\frac{\omega^{2}}{\gamma^{2}}+\Lambda^{2}}\right)\right]^{2} .
$$

Diferente do primeiro caso, este fluxo não diverge em pequeno $b$. Portanto podemos tomar o limite inferior das integrais em $b$ como sendo zero.

A Fig. 4.4 mostra a comparação entre esses dois fluxos contra $b$. O fluxo do monopolo (linha cheia) vai diminuindo para $b$ muito pequeno, enquanto o fluxo para $F\left(Q^{2}\right)=1$ (linha tracejada) tende a infinito no mesmo limite. Apresentamos também uma linha pontilhada que denota onde é feito o corte para o fluxo com $F\left(Q^{2}\right)=1$. Podemos observar, contudo, que os dois fluxos concordam para $b$ suficientemente grande $\left(b \gtrsim R_{A}\right)$.

Por fim, apresentamos o fluxo dependente de $b$ para prótons. Neste caso, consideramos um fator de forma do tipo dipolo elétrico dado por

$$
F(q)=\frac{\Lambda^{4}}{\left(\Lambda^{2}+q^{2}\right)^{2}} ; \Lambda^{2}=0.71 \mathrm{GeV}^{2}
$$

Tal escolha leva ao seguinte fluxo de fótons

$$
\begin{array}{r}
N(\omega, b)=\frac{\alpha_{e m}}{\pi^{2}} \frac{1}{\omega}\left[\frac{\omega}{\gamma} K_{1}\left(\frac{b \omega}{\gamma}\right)-\sqrt{\left(\Lambda^{2}+\frac{\omega^{2}}{\gamma^{2}}\right)} K_{1}\left(b \sqrt{\left(\Lambda^{2}+\frac{\omega^{2}}{\gamma^{2}}\right)}\right)\right. \\
\left.-\frac{b \Lambda^{2}}{2} K_{0}\left(b \sqrt{\left(\Lambda^{2}+\frac{\omega^{2}}{\gamma^{2}}\right)}\right)\right]^{2}
\end{array}
$$

As equações (4.23) e (4.25), foram derivadas no apêndice C.

\subsection{Produção exclusiva de mésons vetoriais em colisões ultraperiféricas}

Nos capítulos 2 e 3 apresentamos o DIS inclusivo como uma ferramenta importante para o estudo da estrutura dos hádrons e apontamos diversas abordagens para estudar este processo em altas energias. Em particular, os experimentos de DIS realizados no HERA fornecem várias informações sobre as funções de estrutura e as seções de choque [70]. Com os dados do HERA foi possível determinar as funções de distribuição dos partons (PDFs) através de análises globais. Foi ainda possível determinar os parâmetros dos modelos fenomenológicos para as seções de choque de dipolo.

No HERA foi observado que aproximadamente $10 \%$ dos eventos que ocorriam deixavam os prótons intactos e separados do estado final produzido por regiões do acelerador onde não havia produção de partículas (grandes gaps de rapidez) [71]. Tais eventos são chamados de difrativos [18]. Em mais baixa ordem em QCD e altas energias, tais eventos são caracterizados pela troca de dois glúons num estado singleto de cor (Pomeron perturbativo), 


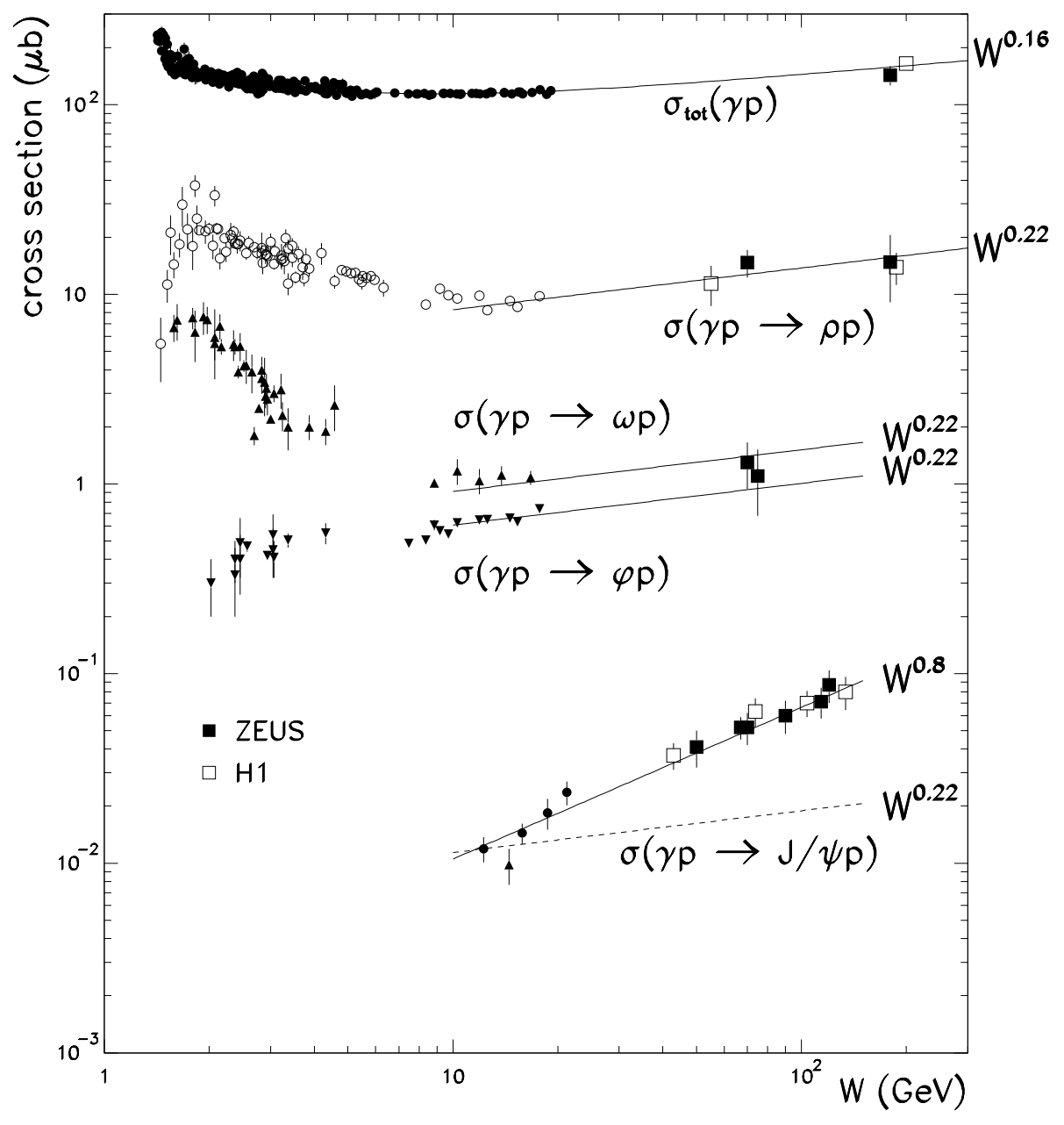

Fig. 4.5: Dependência das seções de choque de produção de mésons vetoriais com a energia de centro de massa $\gamma$ p comparadas com dados de HERA (retirado da Ref.[70]).

tornando o processo mais sensível ao fenômeno de saturação do que eventos onde ocorre a troca de apenas um glúon. Esta característica torna os eventos difrativos extremamente interessantes para o estudo da física não linear.

Entre os eventos difrativos, a produção exclusiva de mésons vetoriais forma uma importante classe que foi estudada no HERA. Para o processo de fotoprodução, que é o processo de interesse nesta tese, temos

$$
\gamma\left(Q^{2} \approx 0\right)+p \rightarrow V+p
$$

onde $V$ denota o méson vetorial produzido. A Fig. 4.5 mostra as seções de choque de fotoprodução exclusiva de diferentes mésons vetoriais como função da energia do centro de 


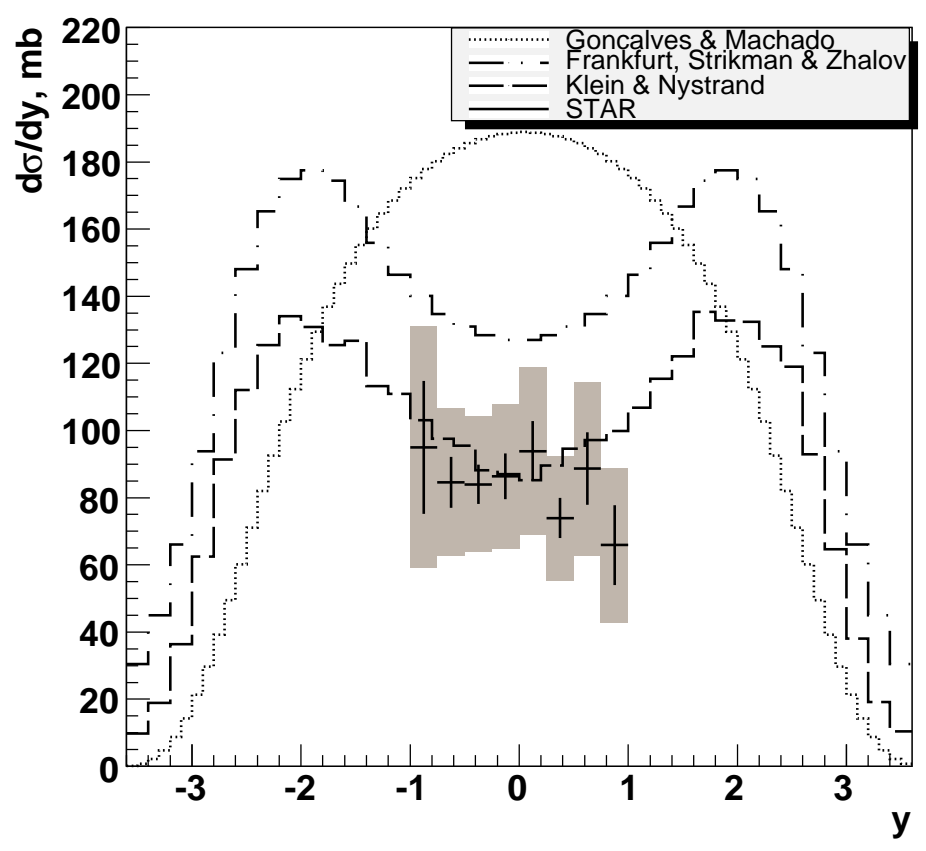

Fig. 4.6: Distribuição de rapidez para a fotoprodução de $\rho$ em colisões ultraperiféricas AuAu no RHIC.

massa $\gamma p$ comparadas com dados do HERA [70]. Em mais baixa ordem em $\alpha_{S}$ (troca de dois glúons), a seção de choque $\gamma p \rightarrow V p$ é dada por $[18,59]$

$$
\frac{\mathrm{d} \sigma}{\mathrm{d} t} \propto\left[\alpha_{S} x_{\mathbb{P}} g\left(x_{\mathbb{P}}, \frac{M_{V}^{2}}{4}\right)\right]^{2},
$$

onde $x_{\mathbb{P}}$ é a fração de momento do próton carregada pelo Pomeron e $g\left(x_{\mathbb{P}}, \frac{M_{V}^{2}}{4}\right)$ é a função de distribuição de glúons. Cabe salientar que essa aproximação é justificada apenas se a massa do méson for grande o suficiente para que seja possível o cálculo com QCD perturbativa. Um cálculo para mésons leves só é possível se $Q^{2} \neq 0$ (de forma que a escala hard é dada por $\left.Q^{2} \rightarrow\left(Q^{2}+M_{V}^{2}\right) / 4\right)$ e é grande o suficiente para comportar métodos perturbativos. Contudo, como estamos interessados em explorar o método de fótons equivalentes, um cálculo desse tipo se afasta dos objetivos dessa tese. Uma característica importante da produção exclusiva de mésons vetoriais é o fato da seção de choque diferencial ser proporcional ao quadrado da distribuição de glúons. Isto torna possível a medida direta de $g\left(x_{\mathbb{P}}, \frac{M_{V}^{2}}{4}\right)$, algo que não é possível no DIS inclusivo. Além disso, 
processos de fotoprodução possibilitam o estudo em virtualidades muito baixas e pequeno $x$, onde efeitos de saturação devem ser importantes.

Em futuros colisores ep $(A)$, o DIS inclusivo e difrativo poderão ser estudados em condições cinemáticas extremas comparadas ao HERA [71]. Em particular, este é o cenário ideal para estudar fenômenos de saturação na colisão entre um sistema diluído (elétron) e um CGC (próton ou núcleo). Em particular, nestas condições espera-se que, para alguns observáveis, a física não linear possa ser separada mais facilmente da física linear [71, 72].

Uma alternativa aos colisores $e p(A)$, são colisores hadrônicos. Em colisores hadrônicos, os processos de fotoprodução podem ser estudados através das colisões ultraperiféricas. Os primeiros estudos experimentais desse tipo foram feitos na última década no RHIC [73, 74], enquanto que os primeiros estudos teóricos com respeito ao tema podem ser encontrados nas Refs. $[75,76,77,78]$, as quais lidam com o processo utilizando diferentes aproximações para a seção de choque $\gamma$-alvo. Em particular, na Ref.[78] é utilizado o formalismo de dipolo de cor, fazendo da mesma uma das principais referências de nossos trabalhos. A Fig. 4.6 mostra a distribuição de rapidez de mesons $\rho$ criados por fotoprodução em colisões ultraperiféricas $A u A u$ no RHIC. Os pontos experimentais (Ref.[74]) são comparados com previsões teóricas (Refs.[75, 78, 79]). Atualmente, o LHC vem medindo a produção de mésons vetoriais e provando energias ainda mais altas do que o RHIC (como pode ser observado na Tab. 4.1). Alguns resultados para a produção exclusiva de partículas no LHC serão brevemente apresentados na próxima seção e, para o caso particular de produção de mésons vetoriais, no próximo capítulo.

\subsection{Produção de partículas em processos foto-induzidos no LHC}

A produção de partículas em processos foto-induzidos em colisores hadrônicos já havia sido estudada no RHIC (conforme foi brevemente discutido na seção anterior) e no Tevatron. Atualmente, o estudo experimental desse tipo de processo, vem sendo feito pelo LHC em energias maiores do que as de seus antecessores. Nessa seção, vamos apresentar alguns resultados para a produção de partículas em processos foto-induzidos no LHC.

Primeiramente, começamos com a produção de pares de léptons e de bósons vetoriais em colisões $p p$. Esses processos ocorrem através dos subprocessos $\gamma \gamma \rightarrow l^{+} l^{-}$(onde $l$ denota um lépton) e $\gamma \gamma \rightarrow W^{+} W^{-}$. A Fig.4.7 (painel esquerdo) mostra dados experimentais da colaboração CMS [80] para a distribuição de $p_{T}$ de pares de múons produzidos em colisões $p p$ em $\sqrt{s}=7 \mathrm{TeV}$. Na Fig.4.7 (painel direito), apresentamos dados experimentais da colaboração CMS [81] para a produção de $\mu^{ \pm} e^{\mp}$ através do processo

$$
p p \rightarrow p^{*} W^{+} W^{-} p^{*} \rightarrow p^{*} \mu^{ \pm} e^{\mp} p^{*}
$$

onde $p^{*}$ significa que a probabilidade de dissociação do próton é levada em conta.

Nas Figs.4.8, apresentamos dados para a produção de $J / \psi$ (painel esquerdo) e de $\rho$ (painel direito) em colisões ultraperiféricas $\mathrm{PbPb}$ em $\sqrt{s}=2,76 \mathrm{TeV}$. Estes resultados estão diretamente relacionados com os temas dessa tese. Em particular, no próximo capítulo, onde 

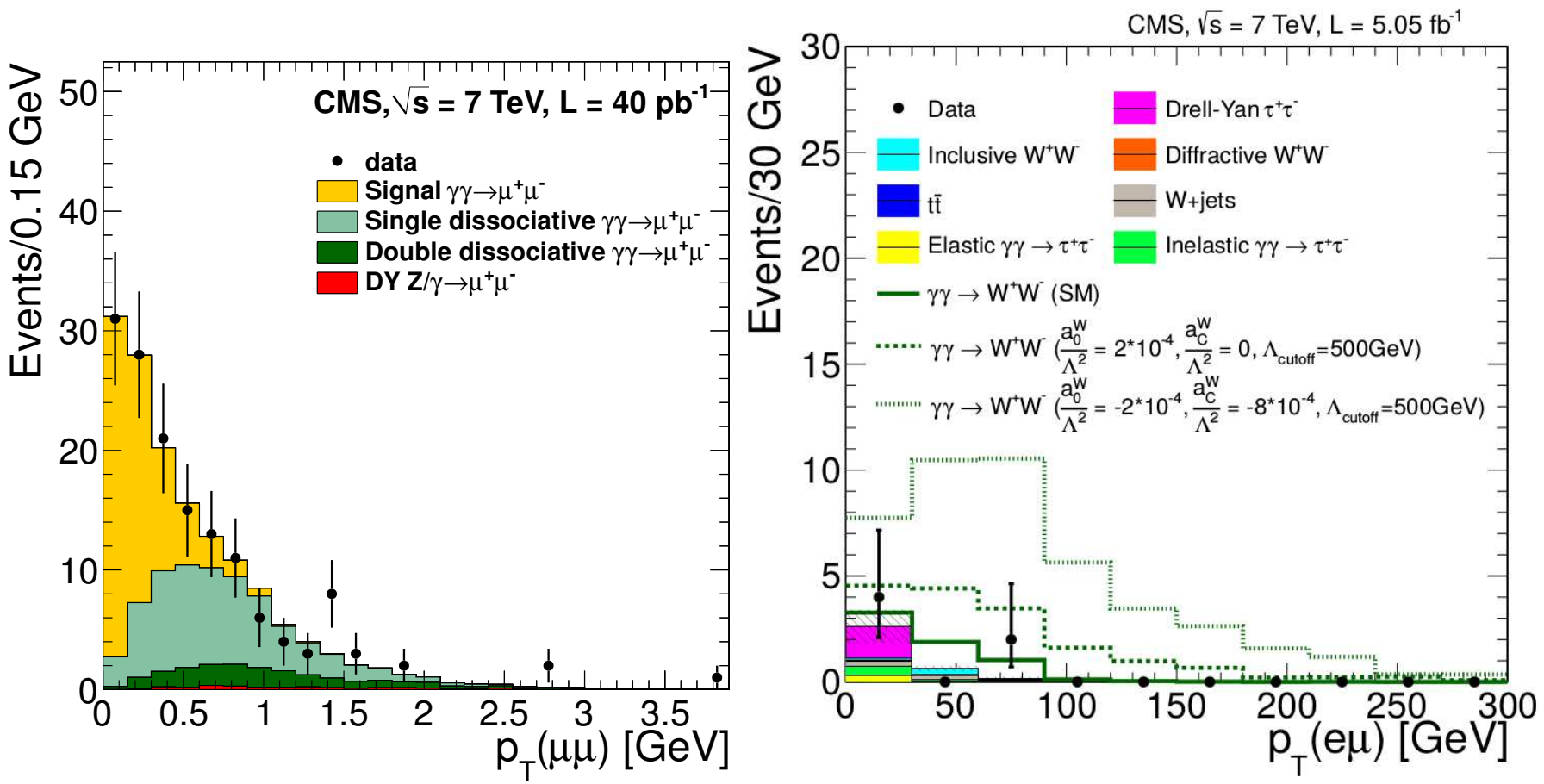

Fig. 4.7: Distribuições de $p_{T}$ para a produção de $\mu^{+} \mu^{-}$[80] (painel esquerdo) e de $\mu^{ \pm} e^{\mp}$ [81] (painel direito) em colisões pp com $\sqrt{s}=7 \mathrm{TeV}$ em interações $\gamma \gamma$. Dados da colaboração CMS.

começaremos a expor nossos primeiros resultados, apresentaremos mais dados experimentais relacionados à produção exclusiva de mésons vetoriais. Mais resultados e maiores detalhes com respeito aos processos que foram discutidos aqui, podem ser encontrados nas Refs.[83, 84].

\subsection{Conclusão}

Neste capítulo apresentamos os principais ingredientes usados na aproximação de fótons equivalentes, iniciando pela discussão do fluxo de fótons gerados por uma carga puntual ultra-relativística. Apresentamos também, as equações dos fluxos de fótons equivalentes associados à prótons e núcleos, que foram amplamente usadas em nossos trabalhos. Em particular, esse método se torna adequado para o estudo de interações ultraperiféricas entre dois hádrons em altas energias, que é um dos processos de interesse nesta tese. Em nossos trabalhos, a aproximação de fótons equivalentes foi usada para estudar interações hádronhádron em termos de interações fóton-hádron e fóton-fóton. As interações fóton-hádron e 

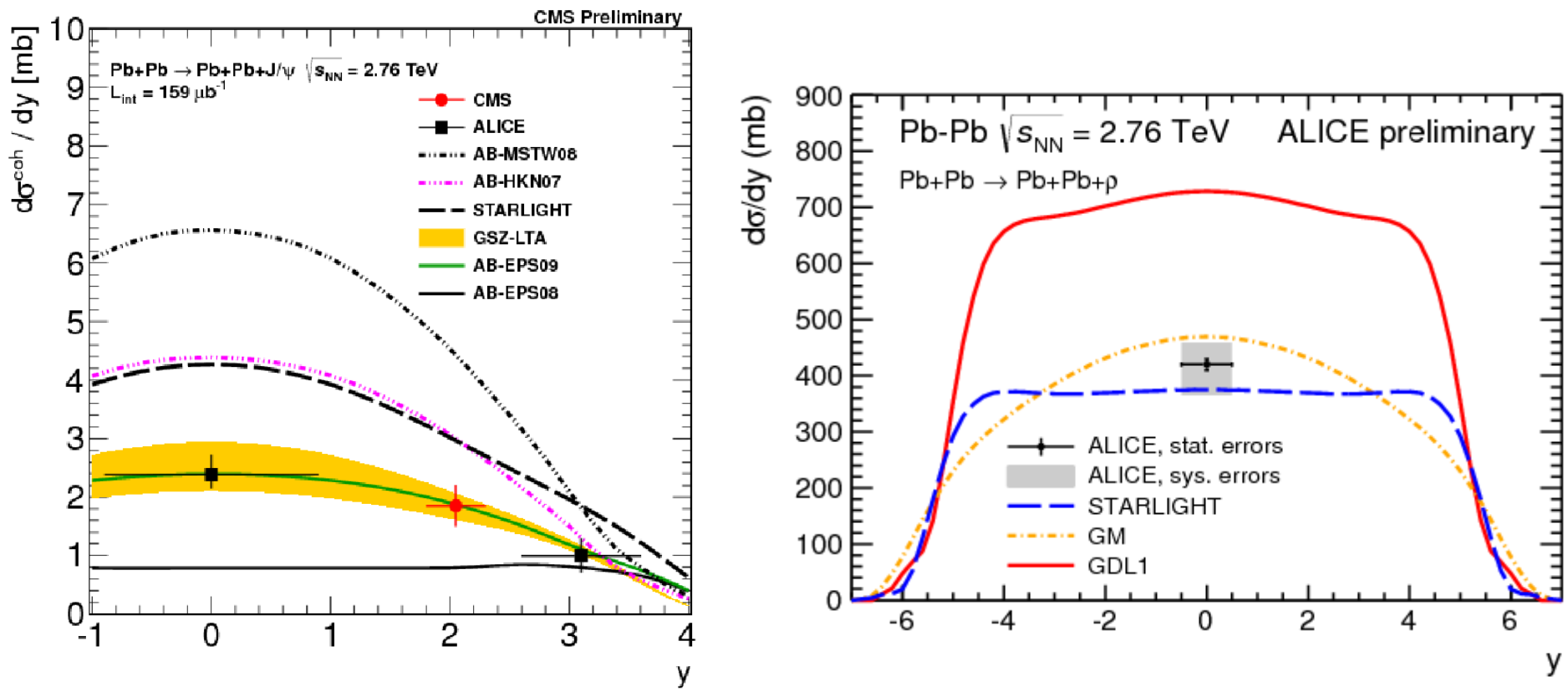

Fig. 4.8: Distribuições de rapidez para a produção de $J / \psi$ [82, 83] (painel esuqerdo) e de $\rho$ [83] (painel direito) em colisões ultraperiféricas $\mathrm{PbPb}$ com $\sqrt{s}=2.76 \mathrm{TeV}$. Dados das colaborações ALICE e CMS.

fóton-fóton por outro lado, foram estudadas através do formalismo de dipolo de cor, que foi discutido no cap.3 para o caso inclusivo e que será estendido nos próximos capítulos para o caso de produção difrativa e exclusiva de mésons vetoriais. Portanto, juntamente com o formalismo de dipolo, a aproximação de fótons equivalentes é uma das principais ferramentas utilizadas nesta tese. Além disso, iniciamos aqui a discussão sobre a produção exclusiva de mésons vetoriais buscando fazer uma breve contextualização de como esse processo vem sendo estudado desde o HERA até o LHC. Até aqui, apresentamos os principais elementos para o estudo da física em altas energias em colisões ultraperiféricas entre dois hádrons. Nos próximos capítulos, apresentaremos nossos resultados para a produção exclusiva de mésons vetoriais em colisões hadrônicas ultraperiféricas em vários processos. 


\section{Capítulo 5}

\section{Fotoprodução difrativa de mésons vetoriais em interações $\gamma h$}

Neste capítulo, apresentamos nossos resultados para a fotoprodução $\left(Q^{2} \approx 0\right)$ exclusiva de mésons vetoriais no processo quase elástico $\gamma^{*} p \rightarrow V p$. Como veremos, tal processo é mediado pela interação fóton-Pomeron. O Pomeron é um objeto que não carrega cor líquida, o que faz com que a emissão de hádrons seja suprimida durante a interação. Como consequência de tal supressão surgem "gaps" de rapidez, que são regiões do espaço de fase onde nenhuma partícula é observada.

O processo físico descrito acima é chamado na literatura de processo difrativo. Sistemas produzidos via difração são caracterizados por portarem os mesmos números quânticos da partícula inicial e são caracterizados por um momento transferido muito pequeno $\left(\sim 1 / R^{2}\right.$, onde $R$ é o raio do alvo). Portanto, em princípio, difração é um processo não perturbativo. Entretanto, uma das principais características da física de saturação é a existência de uma escala de saturação $Q_{s}(x)$ que, em altas energias, pode ser grande o suficiente para que métodos perturbativos possam ser utilizados. Isso significa que o estudo da difração pode ser um teste da física de saturação.

Para começar nosso estudo, vamos analisar a Fig. 5.1, onde mostramos o diagrama que representa o DIS difrativo (DDIS). Na parte superior do diagrama, um elétron emite um fóton que interage com o próton (parte inferior do diagrama). No DIS convencional, o fóton virtual iria interagir com um párton do próton, que carrega carga de cor. No DIS difrativo, o próton emite um Pomeron, denotado pela linha duplamente ondulada na figura, que interage com o fóton virtual e produz um sistema com momento $P_{X}$, enquanto o próton permanece intacto. Em mais baixa ordem em teoria de perturbação, o Pomeron pode ser descrito por dois glúons no estado singleto de cor. As correções de altas energias fazem com que os dois glúons sejam descritos por uma escada de glúons, descrita pela dinâmica BK.

Estamos interessados na produção exclusiva (ou seja, tal que todas as partículas produzidas são identificadas no estado final), onde o sistema de partículas com momento $P_{X}$ é simplesmente um méson vetorial. Além das variáveis cinemáticas do DIS convencional, definimos as seguintes varáveis importantes para a descrição do DIS difrativo:

$$
t=-\left(P^{\prime}-P\right)^{2}
$$




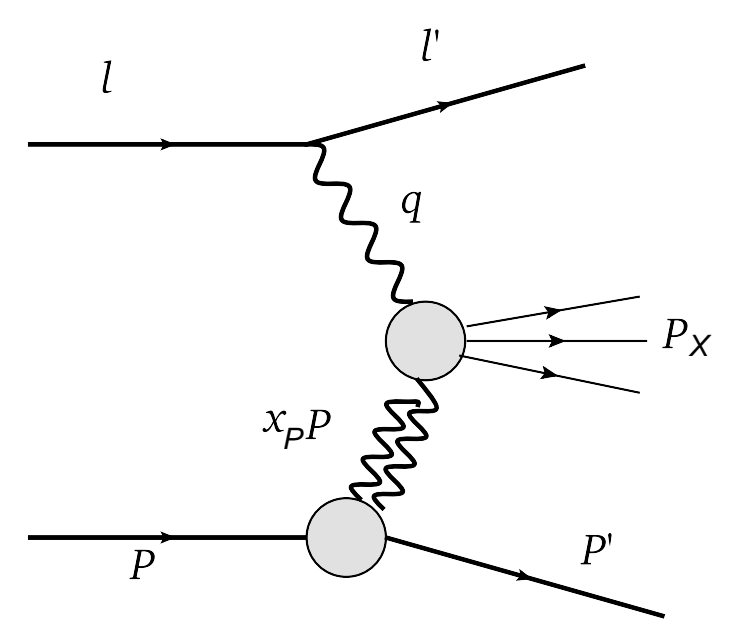

Fig. 5.1: Cinemática do DIS difrativo.

e

$$
x_{\mathbb{P}}=\frac{Q^{2}+M_{V}^{2}}{Q^{2}+W^{2}}
$$

onde $t$ é o quadrado do momentum transferido do alvo e $x_{\mathbb{P}}$ é a fração de momento do alvo carregada pelo Pomeron. Além disso, $W^{2}$ é a energia de centro de massa ao quadrado do sistema $\gamma^{*} p$ e $M_{V}$ é a massa do méson vetorial produzido.

Com esta breve introdução, estamos aptos a estudar a produção exclusiva de mésons vetoriais em colisores hadrônicos. Este processo ocorre numa colisão periférica entre dois hádrons que pode ser separada em dois subprocessos: (i) inicialmente, os hádrons atuam como fontes de fótons quase reais, descritas pela aproximação de fótons equivalentes, e (ii) logo após ocorrem interações $\gamma$-alvo, que são calculadas com o formalismo de dipolo de cor.

No capítulo 3 apresentamos o formalismo de dipolo de cor para o DIS inclusivo. Neste capítulo começamos com a descrição do formalismo de dipolo de cor para o processo quase elástico $\gamma^{*} p(\mathrm{~Pb}) \rightarrow V p(\mathrm{~Pb})$. Em seguida, apresentamos nossos resultados para a produção de mésons vetoriais devido à esse processo e também ao processo $h_{1} h_{2} \rightarrow h_{1} V h_{2}$, onde $h_{i}=p, P b$.

\subsection{Formalismo de dipolo para a produção quase elástica de mésons vetoriais}



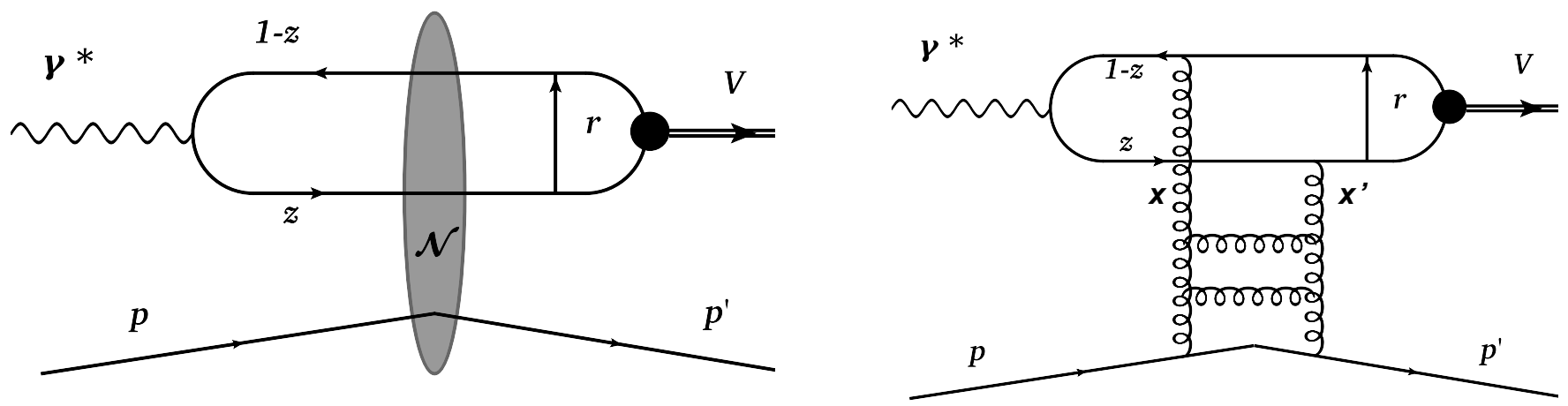

Fig. 5.2: Amplitude de espalhamento quase elástica $\gamma^{*} p \rightarrow V p$.

Nessa seção vamos desenvolver o formalismo para a produção de mésons vetoriais no processo quase elástico $\gamma^{*} p \rightarrow V p$ representado pelos diagramas da Fig. 5.2 (para isso, seguimos a Ref. [31]). O primeiro diagrama da figura mostra um fóton virtual que flutua num dipolo de cor e posteriormente interage com o alvo. Essa interação é descrita pelo blob na figura que representa a amplitude de espalhamento frontal $\mathcal{N}$ entre o dipolo e o próton. O segundo diagrama nos mostra como essa interação pode ser entendida como a troca de uma escada de glúons.

Seguindo a Ref. [31], a amplitude de espalhamento para esse processo é escrita no formalismo de dipolo como (ver Eq. (3.1))

$$
\begin{aligned}
\mathcal{A}_{T, L}^{\gamma^{*} p \rightarrow V p}(x, Q, \Delta) & =\int \mathrm{d}^{2} \vec{r} \int \mathrm{d} z\left(\psi_{V}^{*} \psi\right)_{T, L} \mathcal{A}_{q \bar{q}}(x, r, \Delta) \\
& =i \int \mathrm{d}^{2} \vec{r} \int \mathrm{d} z \int \mathrm{d}^{2} \vec{b}\left(\psi_{V}^{*} \psi\right)_{T, L} e^{-i \vec{b} \cdot \vec{\Delta}} 2[1-S(x, r, b)]
\end{aligned}
$$

onde no segundo sinal de igualdade usamos a Eq. (3.5). Por outro lado, se consideramos que $S$ é predominantemente real, podemos usar a Eq. (3.11) para escrever

$$
\mathcal{A}_{T, L}^{\gamma^{*} p \rightarrow V p}(x, Q, \Delta)=\int \mathrm{d}^{2} \vec{r} \int \mathrm{d} z \int \mathrm{d}^{2} \vec{b}\left(\psi_{V}^{*} \psi\right)_{T, L} e^{-i \vec{b} \cdot \vec{\Delta}} 2 \mathcal{N}(x, r, b)
$$

$\mathrm{Na}$ equação acima $\psi_{V}^{*} \psi$ denota o overlap entre as funções de onda do fóton virtual e do méson vetorial, que é discutida em maiores detalhes no apêndice A e na Ref. [31]. Em particular, $\psi_{V}$ é derivada levando-se em conta que a separação do par $q \bar{q}$ é muito menor do que as dimensões do próton. As funções $\psi_{V}$ e $\psi$ são chamadas de funções de onda frontais. As correções não frontais para a função de onda podem ser levadas em conta incluindo um fator multiplicativo no integrando do tipo $\exp [i(1-z) \vec{r} \cdot \vec{\Delta}]$ (conforme Ref. [85]). 
A seção de choque para o processo quase elástico $\gamma^{*} p \rightarrow V p$ é dada por

$$
\begin{aligned}
\frac{\mathrm{d} \sigma_{T, L}^{\gamma^{*} p \rightarrow V p}}{\mathrm{~d} t} & =\frac{R_{g}^{2}\left(1+\beta^{2}\right)}{16 \pi}\left|\mathcal{A}_{T, L}^{\gamma^{*} p \rightarrow V p}(x, Q, \Delta)\right|^{2} \\
& =\frac{R_{g}^{2}\left(1+\beta^{2}\right)}{16 \pi}\left|\int \mathrm{d}^{2} \vec{r} \int \mathrm{d} z \int \mathrm{d}^{2} \vec{b}\left(\psi_{V}^{*} \psi\right)_{T, L} e^{-i[\vec{b}-(1-z) \vec{r}] \cdot \vec{\Delta}} 2 \mathcal{N}(x, r, b)\right|^{2}
\end{aligned}
$$

$\mathrm{Na}$ equação acima foram incluídos dois fatores de correção. Na obtenção da Eq. (5.4), assumimos que a matriz $S$ era puramente real (e portanto, $\mathcal{A}$ é puramente imaginária). Isto é corrigido multiplicando o lado direito da equação acima por $\left(1+\beta^{2}\right)$, onde

$$
\beta=\frac{\operatorname{Re} \mathcal{A}}{\operatorname{Im} \mathcal{A}}=\tan \left(\frac{\pi \lambda}{2}\right), \lambda=\frac{\partial \ln \left[\mathcal{A}\left(x, Q^{2}, \Delta\right)\right]}{\partial \ln (1 / x)} .
$$

Além disso, os glúons ligados ao par $q \bar{q}$ podem portar diferentes frações de momento do próton $x$ e $x^{\prime}$ (conforme diagrama à direita da Fig. 5.2). No limite em que $x^{\prime} \ll x \ll 1$ e $t$ é pequeno, temos [86]

$$
R_{g}(\lambda)=\frac{2^{2 \lambda+3}}{\sqrt{\pi}} \frac{\Gamma(\lambda+5 / 2)}{\Gamma(\lambda+4)} .
$$

Em alguns modelos de dipolo para $\mathcal{N}$ não conhecemos a dependência em $b$. Para esses modelos, é assumido que a seguinte parametrização seja válida

$$
\mathcal{N}(x, r, b)=S(b) \mathcal{N}(x, r)
$$

de forma que

$$
\begin{aligned}
\sigma^{d i p}(x, r) & =\int \mathrm{d}^{2} b \mathcal{N}(x, r, b) \\
& =\mathcal{N}(x, r) \int \mathrm{d}^{2} b S(b) \\
& =\sigma_{0} \mathcal{N}(x, r),
\end{aligned}
$$

onde $\sigma_{0}$ é um parâmetro livre de cada modelo obtido através de ajuste aos dados experimentais.

É possível assumir a seguinte dependência em $t$ para a seção de choque

$$
\begin{aligned}
\sigma & =\left.\int_{-\infty}^{0} \mathrm{~d} t e^{-B|t|} \frac{\mathrm{d} \sigma}{\mathrm{d} t}\right|_{t=0} \\
& =\left.\frac{\mathrm{d} \sigma}{\mathrm{d} t}\right|_{t=0} \int_{-\infty}^{0} \mathrm{~d} t e^{B t} \\
& =\left.\frac{1}{B} \frac{\mathrm{d} \sigma}{\mathrm{d} t}\right|_{t=0}
\end{aligned}
$$

onde $B$ é chamado de parâmetro de slope. Existem parametrizações experimentais para $B$ que dependem da massa do méson vetorial produzido, de $Q^{2}$ e/ou de $W^{2}$. Para modelos fenomenológicos de $\mathcal{N}$ sem dependência em $b$, a parametrização acima precisa ser necessariamente utilizada para cálculos de seções de choque $\gamma p$, enquanto que em modelos com dependência em $b$ (como o bCGC) esta parametrização não é indispensável (embora possa ser utilizada também). 


\subsubsection{Formalismo de dipolo para a produção difrativa de mésons vetoriais em colisões $\gamma A$}

Vamos agora apresentar as principais expressões para a produção de mésons vetoriais num processo quase elástico na interação $\gamma$-núcleo. Neste caso existem dois tipos de processos a serem considerados: processos coerentes e processos incoerentes. No processo coerente, o fóton interage de forma coerente com todos os núcleons do núcleo. Já nos processos incoerentes, o fóton interage com apenas um núcleon do núcleo causando a quebra parcial ou excitação do núcleo. Tal processo é ainda difrativo por não trocar cor e ser caracterizado pela presença de gaps de rapidez. Nessa seção vamos apresentar as principais equações utilizadas em nossos cálculos.

\section{Processos coerentes}

A seção de choque diferencial para a produção coerente pode ser escrita de forma análoga à seção de choque do caso $\gamma^{*} p$

$$
\frac{\mathrm{d} \sigma^{\gamma^{*} A}}{\mathrm{~d} t}=\frac{1}{16 \pi}\left|\mathcal{A}^{\gamma^{*} A}(x, r, \Delta)\right|^{2}
$$

onde o índice $A$ na seção de choque e na amplitude denota a interação $\gamma^{*} A$. Devido à relação entre as varáveis $t$ e $\Delta$ podemos escrever

$$
\mathrm{d} t=\mathrm{d} \vec{\Delta}^{2}
$$

Por outro lado

$$
\mathrm{d}^{2} \vec{\Delta}=2 \pi|\vec{\Delta}| \mathrm{d} \vec{\Delta}=\pi \mathrm{d} \vec{\Delta}^{2}
$$

e nos permite escrever

$$
\mathrm{d} t=\frac{\mathrm{d}^{2} \vec{\Delta}}{\pi} .
$$

Então, a seção de choque pode ser reescrita como

$$
\frac{\mathrm{d} \sigma^{\gamma^{*} A}}{\mathrm{~d}^{2} \vec{\Delta}}=\frac{1}{16 \pi^{2}}\left|\mathcal{A}^{\gamma^{*} A}(x, r, \Delta)\right|^{2} .
$$

De forma análoga ao que foi feito na seção anterior, é possível escrever a amplitude $\gamma^{*} A$ como

$$
\mathcal{A}^{\gamma^{*} A}(x, Q, \Delta)=i \int \mathrm{d}^{2} \vec{r} \mathrm{~d} z\left[\psi_{V}^{*} \psi\right] \int \mathrm{d}^{2} \vec{b} e^{-i \vec{b} \cdot \vec{\Delta}} 2 \mathcal{N}^{A}(x, r, b)
$$

onde $\mathcal{N}^{A}(x, r, b)$ é a parte imaginária da amplitude de espalhamento frontal dipolo-núcleo. Esta quantidade pode ser calculada através do modelo de Glauber em termos da seção de choque dipolo-próton. Neste modelo, considera-se que o espalhamento dipolo-núcleo possa 
ser expresso em termos do espalhamento do dipolo com cada núcleon. Ou seja, os núcleons são tratados como centros espalhadores independentes, de forma que o problema do cálculo da amplitude $\mathcal{N}^{A}$ pode ser reduzido ao cálculo da amplitude de espalhamento para um problema de dois corpos. Seguindo as Refs. [87, 88], temos

$$
\mathcal{N}^{A}(x, r, b)=1-\exp \left[-\frac{1}{2} \sigma_{d i p}\left(x, r^{2}\right) T_{A}(b)\right],
$$

onde $\sigma_{\text {dip }}\left(x, r^{2}\right)$ é a seção de choque dipolo-próton e $T_{A}(b)$ é a função perfil nuclear obtida de uma distribuição de Fermi para a densidade nuclear normalizada a $A$.

Integrando a Eq.(5.15) em $\Delta$ e usando as Eqs. (5.16) e (5.17) obtemos

$$
\sigma^{\gamma^{*} A}=\int \mathrm{d}^{2} \vec{b}\left|\int \mathrm{d}^{2} \vec{r} \int \mathrm{d} z\left[\psi_{V}^{*}(r, z) \psi\left(r, z, Q^{2}\right)\right]\left\{1-\exp \left[-\frac{1}{2} \sigma_{d i p}\left(x, r^{2}\right) T_{A}(b)\right]\right\}\right|^{2} .
$$

Com esta expressão vamos calcular a seção de choque de um processo coerente.

\section{Processos incoerentes}

A seção de choque de um processo incoerente ainda não é bem entendida e existem alguns modelos para calculá-la [89, 90, 91, 92]. Vamos usar o modelo proposto na Ref. [90], que fornece a seguinte seção de choque

$$
\sigma^{\gamma^{*} A \rightarrow V A^{\prime}}=\frac{|\operatorname{Im} \mathcal{A}(s, t=0)|^{2}}{16 \pi B}
$$

onde $B$ é o slope (igual ao caso $\left.\gamma^{*} p\right)$ e

$$
|\operatorname{Im} \mathcal{A}|^{2}=\int \mathrm{d}^{2} \vec{b}\left|\int \mathrm{d}^{2} \vec{r} \int \mathrm{d} z\left[\psi_{V}^{*}(r, z) \psi\left(r, z, Q^{2}\right)\right] \sigma_{d i p} \exp \left[-\frac{1}{2} \sigma_{d i p} T_{A}(b)\right]\right|^{2} .
$$

Com tudo o que foi visto até aqui, temos condições de estudar as seções de choque $\gamma^{*} p(A)$ e colisões ultraperiféricas $p p, p P b$ e $\mathrm{PbPb}$ em energias do LHC. Vamos apresentar nossos resultados a partir da próxima seção.

\subsection{Fotoprodução difrativa de $J / \psi$ em colisões ultraperiféricas}

Nesta seção vamos estudar a fotoprodução difrativa de $J / \psi$ em colisões $p p, p P b$ e $P b P b$, onde nesse último caso tratamos apenas a produção coerente (o caso de produção incoerente será mostrado em uma subseção separada). Vamos considerar energias do LHC e usar o formalismo de dipolo para o cálculo da seção de choque fóton-hádron e a aproximação de fótons equivalentes para o tratamento da seção de choque hádron-hádron. Esses resultados foram publicados na Ref. [1]). 

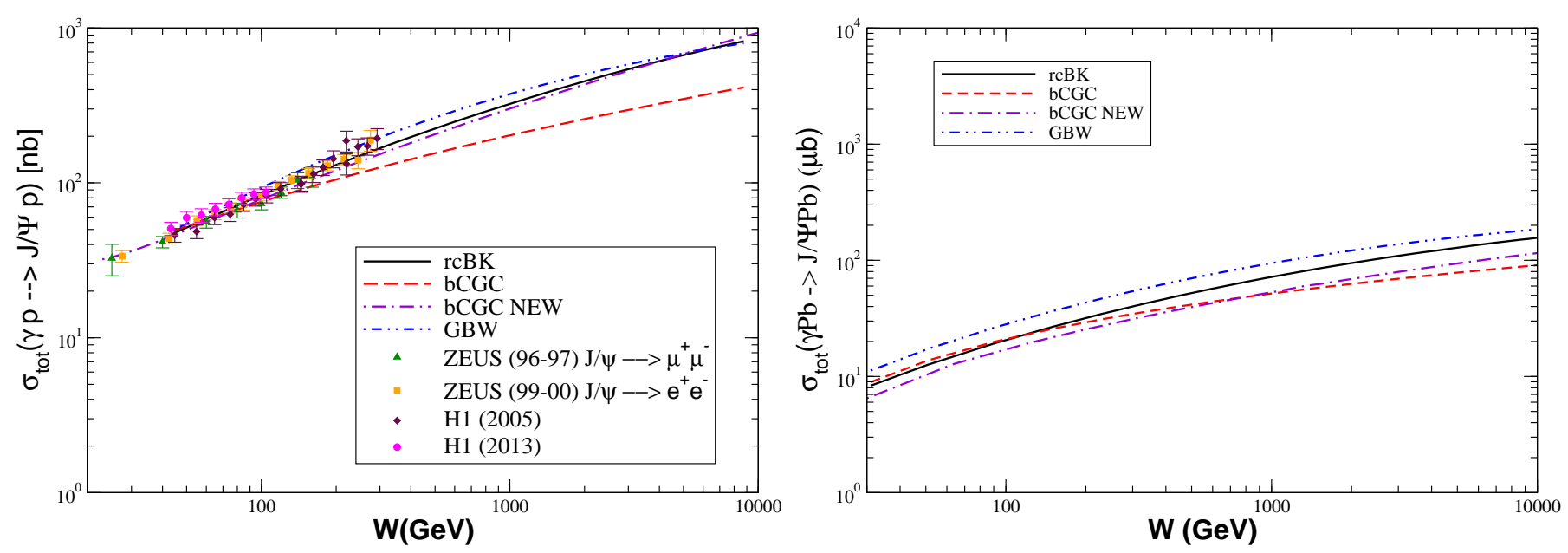

Fig. 5.3: Seção de choque $\gamma p(P b)$ como função da energia do centro de massa $\gamma p(P b)$. $\grave{A}$ esquerda: processo $\gamma^{*} p \rightarrow J / \psi p$. ’̀ direita: $\gamma^{*} \mathrm{~Pb} \rightarrow J / \psi P b$ (coerente).

Nosso principal objetivo é estimar a incerteza teórica das previsões existentes na literatura. Para isso, fixamos um único modelo para a função de onda do méson vetorial (o modelo Gaus-LC) e para o fluxo de fótons equivalentes e calculamos a produção de $J / \psi$ usando diferentes modelos da amplitude de espalhamento $\mathcal{N}$. Nossos resultados serão confrontados com os dados experimentais da colaboração LHCb de fotoprodução de $J / \psi$ em colisões $p p$ a $\sqrt{s}=7 \mathrm{TeV}$ (Refs. [93, 94]) e com dados da colaboração ALICE de colisões $\mathrm{PbPb}$ a $\sqrt{s}=14 \mathrm{TeV}$ (Refs. [82, 95]). Vamos também apresentar nossas previsões para a produção de $J / \psi$ em colisões $p P b$ e para energias do centro de massa mais altas.

No estudo de fotoprodução é interessante considerar uma colisão hádron-hádron ultraperiférica, isto é, uma colisão em que o parâmetro de impacto entre os hádrons seja maior do que a soma de seus raios. Nestas condições, o início da interação é dominado pela interação eletromagética, possibilitando o estudo com o método de fótons equivalentes.

\subsubsection{Colisões $\gamma p$ e $\gamma P b$}

Nesta subseção vamos apresentar nossos resultados para as seções de choque do processo quase elástico $\gamma p(A) \rightarrow V p(A)$. Vamos considerar diferentes modelos para a amplitude dipolo-alvo $\mathcal{N}$. Lembramos que o formalismo usado foi descrito no início deste capítulo e os modelos para $\mathcal{N}$ utilizados foram apresentados no capítulo 3.

A seção de choque do processo $\gamma p(P b)$ é um dos ingredientes básicos nos nossos cálculos. No caso $\gamma p$ utilizamos a Eq. (5.10). Neste caso é necessário conhecer o parâmetro de slope $B$. Este parâmetro foi determinado através da análise dos dados experimentais de HERA [96, 97, 98] (mostrados na Fig. 5.3 à esquerda). 
A Fig. 5.3 mostra nossos resultados para as seções de choque dos processos $\gamma p$ (esquerda) e $\gamma \mathrm{Pb}$ (direita). No caso $\gamma p$, observamos que os modelos de $\mathcal{N}$ utilizados fornecem seções de choque que possuem um comportamento com a energia que é compatível com os dados de HERA de baixas energias. Deste gráfico, podemos perceber que a versão mais recente do modelo bCGC [50] (aqui chamada de bCGC NEW) prevê um crescimento mais rápido da seção de choque com a energia comparado com sua versão original [99], tornando-o consistente com os dados de HERA.

Nossas previsões para o caso $\gamma P b$ são apresentadas na Fig. 5.3, à direita. Podemos observar que os modelos bCGC e rcBK concordam em baixas energias. O limite inferior de nossas previsões é dado pelo modelo bCGC NEW em baixas energias, até $W \approx 500 \mathrm{GeV}$, e pelo modelo bCGC em energias mais altas. Além disso, o modelo GBW fornece o limite superior de nossas previsões em todo o intervalo de energia considerado.

Com o conhecimento do comportamento das seções de choque $\gamma-$ alvo com a energia do centro de massa $\gamma-p(P b)$, podemos estender nossos resultados para colisões $p p, p P b$ e $\mathrm{PbPb}$ em energias do LHC. Esta extensão será discutida na próxima subseção, onde apresentaremos nossos resultados para distribuições de rapidez e seções de choque totais.

\subsubsection{Colisões $\mathrm{pp}, \mathrm{pPb}$ e $\mathrm{PbPb}$}

Um dos observáveis em que estamos interessados é a distribuição de rapidez. Para definila, vamos inicialmente reescrever a equação (4.8) com uma notação mais adequada para o nosso estudo

$$
\sigma^{h_{1} h_{2} \rightarrow h_{1} h_{2} J / \psi}=\int_{\omega_{\min }}^{\infty} \mathrm{d} \omega \frac{n^{h_{2}}(\omega)}{\omega} \sigma^{\gamma h_{1}}(\omega)
$$

com

$$
\omega_{\text {min }}=\frac{W_{\gamma p(A), \min }^{2}}{4 \gamma_{L} m_{p}}, W_{\gamma p(A), \min }=M_{J / \psi}
$$

sendo $\gamma_{L}$ o fator de Lorentz, $m_{p}$ a massa do próton e $M_{J / \psi}$ a massa do $J / \psi$. De forma geral, $W_{\gamma p(A)}^{2}$, que é a energia de centro de massa fóton-alvo ao quadrado, é definida por

$$
W_{\gamma p(A)}^{2}=4 \omega E_{p},
$$

onde $\omega$ é a energia do fóton e $E_{p}$ é a energia do próton. Na Eq.(5.21) estamos considerando uma colisão entre dois hádrons $h_{1}$ e $h_{2}$, onde o hádron $h_{2}$ atua como uma fonte de fótons (esses fótons são associados ao fluxo $n^{h_{2}}(\omega)$ ) que vão interagir com o hádron $h_{1}$ (a seção de choque para a interação fóton- $h_{1}$ é denotada por $\left.\sigma^{\gamma h_{1}}\right)$. A energia do fóton se relaciona com a variável de rapidez $y$ da seguinte forma

$$
\omega=\frac{M_{V}}{2} e^{y}
$$

onde $M_{V}$ é a massa do méson vetorial. Dessa forma, usando a Eq.(5.21), definimos a distribuição de rapidez para o processo como $(\operatorname{com} \mathrm{d} \sigma / \mathrm{d} \omega=(1 / \omega) \mathrm{d} \sigma / \mathrm{d} y)$

$$
\frac{\mathrm{d} \sigma^{h_{1} h_{2} \rightarrow h_{1} h_{2} J / \psi}}{\mathrm{d} y}=n^{h_{2}}(y) \sigma^{\gamma h_{1}}(y) .
$$



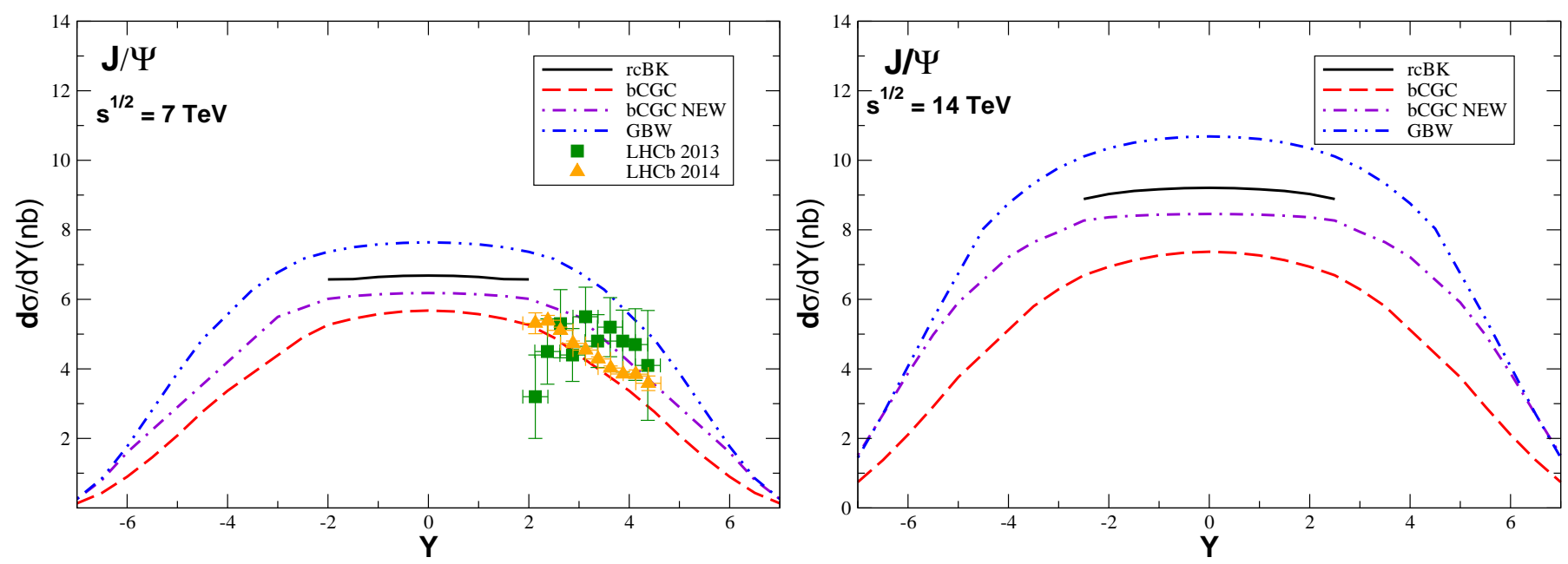

Fig. 5.4: Distribuição de rapidez para a fotoprodução difrativa de $J / \psi$ em colisões pp em

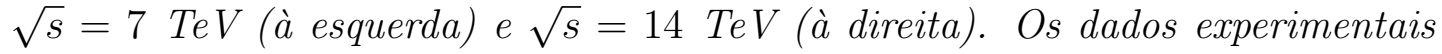
são da colaboração $\mathrm{LHCb}$ [93, 94].

Ao derivar a equação acima, consideramos que o hádron $h_{2}$ atua como fonte de fótons e que o hádron $h_{1}$ é o alvo. Entretanto, numa aproximação mais realista, qualquer um dos hádrons pode atuar como fonte de fótons e/ou alvo. Levando essa outra contribuição em conta, a equação acima fica

$$
\frac{\mathrm{d} \sigma^{h_{1} h_{2} \rightarrow h_{1} h_{2} J / \psi}}{\mathrm{d} y}=n^{h_{2}}(y) \sigma^{\gamma h_{1}}(y)+n^{h_{1}}(-y) \sigma^{\gamma h_{2}}(-y) .
$$

Além disso, com ela podemos calcular a seção de choque total

$$
\sigma^{h_{1} h_{2} \rightarrow h_{1} h_{2} J / \psi}=\int_{y_{\min }}^{y_{\max }} \mathrm{d} y \frac{\mathrm{d} \sigma^{h_{1} h_{2} \rightarrow h_{1} h_{2} J / \psi}}{\mathrm{d} y}
$$

que é outro observável calculado neste trabalho. Em particular, para o cálculo da distribuição de rapidez usamos os fluxos de fótons definidos no cap. 4 para prótons e núcleos. Nos gráficos que serão mostrados a seguir desconsideramos interações soft que levariam a uma produção extra de partículas reduzindo o gap de rapidez do estado final.

Na Fig. 5.4 apresentamos nossos resultados para a distribuição de rapidez de $J / \psi$ 's criados através de fotoprodução difrativa em colisões $p p$ a $\sqrt{s}=7$ (gráfico da esquerda) e nossas previsões para $14 \mathrm{TeV}$ (gráfico da direita) usando os modelos GBW, rcBK, bCGC e bCGC NEW para a amplitude de espalhamento $\mathcal{N}$. Como o modelo rcBK é válido apenas para $x<0.01$ (ou equivalentemente $W>30 \mathrm{GeV}$ ), obtivemos apenas um intervalo limitado em rapidez para a região central. Observamos também que, entre os modelos usados, a maior diferença dos resultados se encontra na região central de rapidez ( $y$ em torno de zero) 

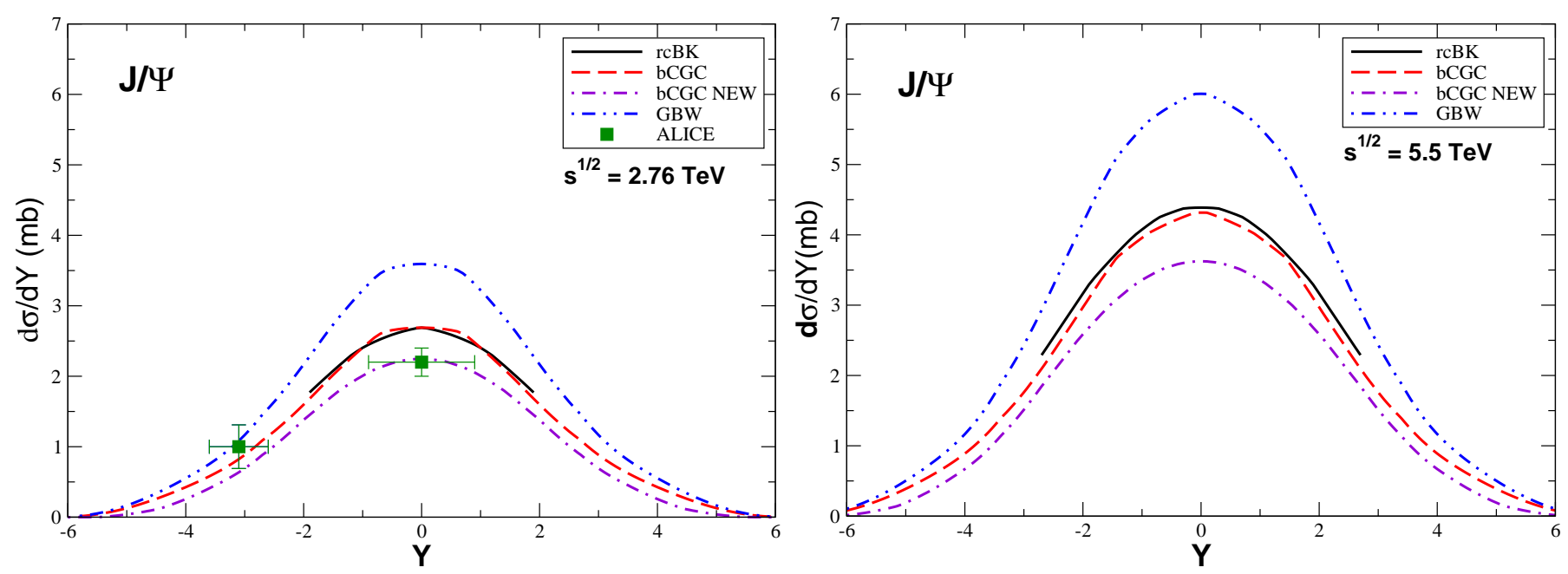

Fig. 5.5: Distribuição de rapidez de $J / \psi$ 's criados através de fotoprodução difrativa em

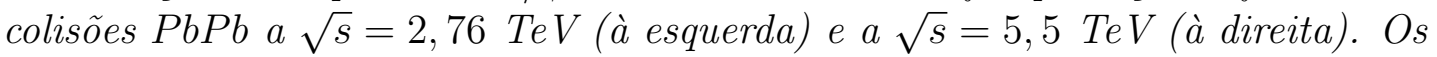
dados experimentais são da colaboração ALICE [82, 95].

e que o modelo GBW pode ser tomado como limite superior para a distribuição de rapidez enquanto o modelo bCGC pode ser tomado como limite inferior. No caso de $\sqrt{s}=7 \mathrm{TeV}$, comparando nossos resultados com os dados experimentais de LHCb [93, 94], vemos que os modelos bCGC e bCGC NEW estão em boa concordância com a experiência. Além disso, notamos que com o aumento de $\sqrt{s}$, nossas previsões mostram que as diferenças entre os modelos também devem aumentar. Essa é uma boa motivação para um futuro estudo experimental em energias mais altas, o que possibilitaria um melhor entendimento da QCD no regime de altas energias.

Na Fig. 5.5 apresentamos nossos resultados da distribuição de rapidez para a produção coerente de $J / \psi$ em colisões $P b P b$ em $\sqrt{s}=2,76$ e 5,5 TeV. Assim como no caso anterior para colisões $p p$, observamos que as maiores discrepâncias entre os modelos de $\mathcal{N}$ ocorrem na região central de rapidez. Podemos observar que, para $\sqrt{s}=2,76 \mathrm{TeV}$, o modelo bCGC NEW parece dar uma boa descrição dos dados da colaboração ALICE [93, 94]. Em particular, observamos que o modelo rcBK não descreve o ponto experimental na região central de rapidez. Novamente, como no caso pp, um aumento na escala de energia provoca um aumento da separação entre as previsões dos modelos na região de rapidez central.

Na Fig. 5.6, apresentamos nossas previsões para a distribuição de rapidez para a fotoprodução difrativa de $J / \psi$ em colisões $p P b$ em $\sqrt{s}=5 \mathrm{TeV}$. Como esperado, temos uma distribuição de rapidez assimétrica em torno de $Y=0$.

Por fim, a Tab. 5.1 mostra nossos resultados para a seção de choque total para a fotoprodução difrativa de $J / \psi$ em colisões $p p, p P b$ e $P b P b$. Como esperado de nossa análise da distribuição de rapidez, os valores das seções de choque são bastante diferentes. 


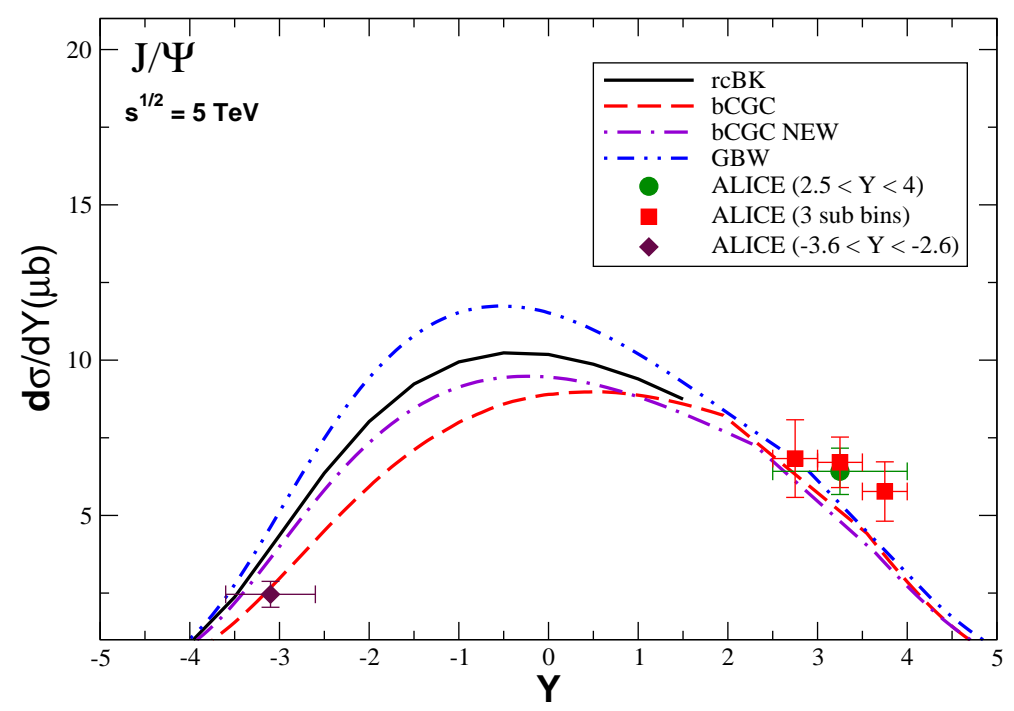

Fig. 5.6: Distribuição de rapidez para a fotoprodução difrativa de $J / \psi$ em colisões pPb em $\sqrt{s}=5 \mathrm{TeV}$. Os dados da colaboração ALICE podem ser encontrados na Ref.[100].

\begin{tabular}{||c|c|c|c||}
\hline \hline & GBW & bCGC & bCGC NEW \\
\hline$p p(\sqrt{s}=7 \mathrm{TeV})$ & $74.0 \mathrm{nb}$ & $49.0 \mathrm{nb}$ & $59.0 \mathrm{nb}$ \\
\hline$p p(\sqrt{s}=14 \mathrm{TeV})$ & $113.0 \mathrm{nb}$ & $71.2 \mathrm{nb}$ & $93.7 \mathrm{nb}$ \\
\hline \hline$p P b(\sqrt{s}=5 \mathrm{TeV})$ & $67.11 \mu \mathrm{b}$ & $52.38 \mu \mathrm{b}$ & $55.98 \mu \mathrm{b}$ \\
\hline \hline$P b P b(\sqrt{s}=2.76 \mathrm{TeV})$ & $18.2 \mathrm{mb}$ & $13.6 \mathrm{mb}$ & $11.0 \mathrm{mb}$ \\
\hline$P b P b(\sqrt{s}=5.5 \mathrm{TeV})$ & $33.8 \mathrm{mb}$ & $24.4 \mathrm{mb}$ & $20.3 \mathrm{mb}$ \\
\hline \hline
\end{tabular}

Tab. 5.1: Seção de choque total para a produção difrativa de $\mathrm{J} / \psi$ em colisões $p p, p P b$ e $\mathrm{PbPb}$ em energias do $\mathrm{LHC}$.

\subsubsection{Fotoprodução difrativa incoerente de $J / \psi$ em colisões $P b P b$}

Nesta seção vamos mostrar nossos resultados para a fotoprodução difrativa incoerente de $J / \psi$, considerando dois modelos para a amplitude de espalhamento dipolo-próton (GBW e bCGC NEW) e os dois modelos para as funções de onda de mésons vetoriais (Gaus-LC e Boosted Gaussian) discutidos no apêndice A. Novamente usamos o que foi visto na subseção 5.1.1 para o cálculo da produção de $J / \psi$ em colisões fóton-núcleo. Para o slope $B$ usamos 

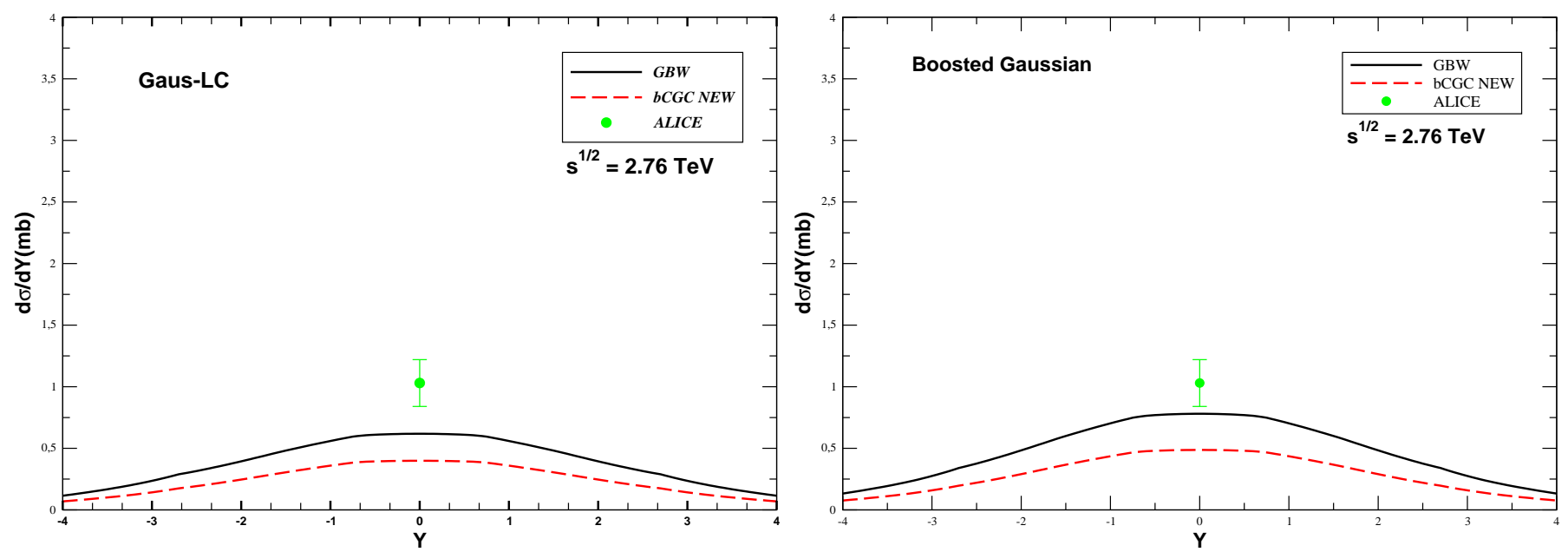

Fig. 5.7: Distribuição de rapidez para a fotoprodução difrativa incoerente de $\mathrm{J} / \psi$ em colisões $\mathrm{PbPb}$ em $\sqrt{s}=2,76 \mathrm{TeV}$. $\grave{A}$ esquerda usamos o modelo Gaus-LC para a função de onda do méson e à direita, o modelo Boosted Gaussian. O dado experimental foi extraído da colaboração ALICE [95].

a parametrização [101]

$$
B=0,60\left[\frac{14}{\left(\frac{Q^{2}+M_{V}^{2}}{\mathrm{GeV}^{2}}\right)^{0,26}}+1\right] \mathrm{GeV}^{-2}
$$

Na Fig. 5.7 apresentamos nossos resultados para a distribuição de rapidez para a fotoprodução difrativa incoerente de $J / \psi$ em colisões $P b P b$ em $\sqrt{s}=2,76 \mathrm{TeV}$ para os modelos GBW, e bCGC NEW para a amplitude de espalhamento $\mathcal{N}$ e os modelos Gaus-LC (à esquerda) e Boosted Gaussian (à direita). Podemos observar que os modelos usados não são capazes de descrever o dado experimental, e que nossa melhor descrição é obtida com o modelo GBW para $\mathcal{N}$ e Boosted Gaussian para $\psi_{V}$.

Por fim, na Fig. 5.8, apresentamos nossos resultados para a distribuição de rapidez para a produção de $J / \psi$ em colisões $P b P b$ a $\sqrt{s}=5.5 \mathrm{TeV}$ com os mesmos modelos para $\mathcal{N}$ e $\psi_{V}$ usados na Fig. 5.7. Podemos observar que o aumento em energia leva a um aumento da diferença entre os modelos de $\mathcal{N}$. Outras aproximações para a produção incoerente de $J / \psi$ podem ser encontrados nas Refs.[89, 91, 92]. 

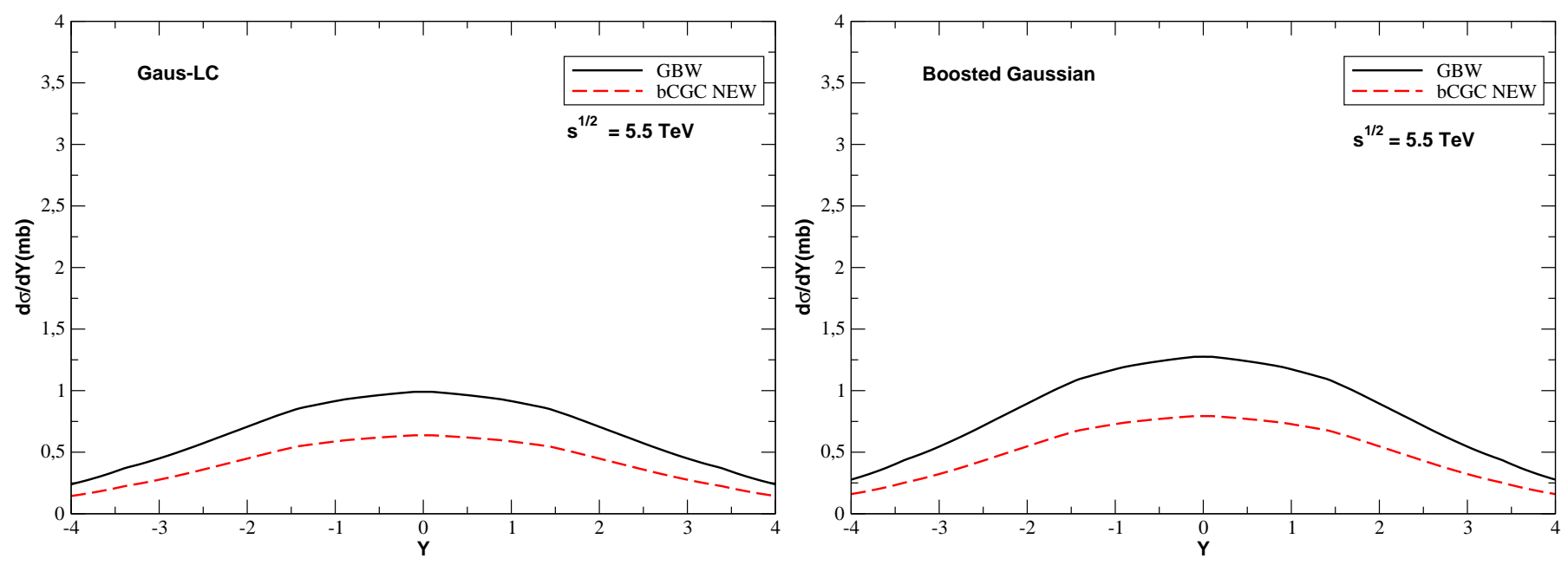

Fig. 5.8: Distribuição de rapidez para a fotoprodução difrativa incoerente de $\mathrm{J} / \psi$ em colisões $\mathrm{PbPb}$ em $\sqrt{\mathrm{s}}=5,5 \mathrm{TeV}$. À esquerda usamos o modelo Gaus-LC para a função de onda do méson e à direita, o modelo Boosted Gaussian.

\subsection{Fotoprodução difrativa de $\Upsilon$ em colisões ultraperiféricas}

Nesta subseção vamos estudar a fotoprodução do méson $\Upsilon$ em colisões ultraperiféricas $p p$ e $p P b$ nas energias do LHC, usando o formalismo de dipolo e a aproximação de fótons equivalentes. Aqui, consideramos diferentes modelos para a amplitude de espalhamento $\mathcal{N}$ e para a função de onda do $\Upsilon$. Nossos objetivos são atualizar os cálculos, apresentar nossas previsões para a produção de $\Upsilon$ e comparar os diferentes modelos de $\mathcal{N}$ e $\psi_{V}$. Assim como no caso da produção de $J / \psi$, calculamos distribuições de rapidez e seções de choque totais. Esses resultados foram publicados na Ref.[2].

\subsubsection{Colisões $\gamma p$}

Os modelos para a amplitude de espalhamento dipolo-próton usados nestes cálculos foram GBW, GBW KSX e bCGC NEW, que chamaremos apenas de bCGC. Os cálculos com o modelo bCGC foram feitos de duas formas diferentes: (i) uma usando o parâmetro de slope que será chamada de $b C G C B_{V}$ e outra (ii) fazendo uma integração sobre $t$ que será chamada de $b C G C$ full. Os modelos GBW, GBW KSX e bCGC $B_{V}$ foram calculados 


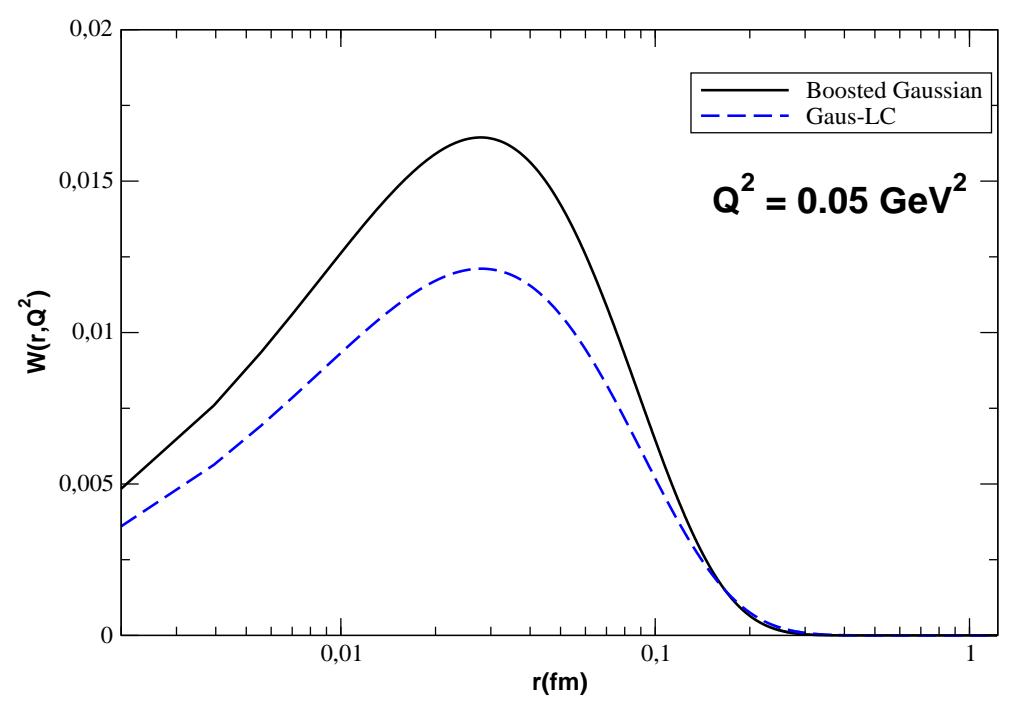

Fig. 5.9: Comparação da parte transversal da função de onda do $\Upsilon$ para os modelos Boosted Gaussian e Gaus-LC.

usando a seguinte parametrização para o slope $B$ [102]

$$
B=N\left[\frac{14}{\left(M_{\Upsilon} / \mathrm{GeV}\right)^{0,4}}+1\right], N=0.55 \mathrm{GeV}^{-2},
$$

enquanto que bCGC full representa a seção de choque calculada pela integração em $t$

$$
\begin{aligned}
\sigma_{T}^{\gamma^{*} p \rightarrow V p} & =\int_{-\infty}^{0} \mathrm{~d} t \frac{\mathrm{d} \sigma_{T}^{\gamma^{*} p \rightarrow V p}}{\mathrm{~d} t} \\
& =\frac{R_{g}^{2}\left(1+\beta^{2}\right)}{16 \pi} \int_{-\infty}^{0} \mathrm{~d} t\left|\mathcal{A}_{T}^{\gamma^{*} p \rightarrow V p}\right|^{2} .
\end{aligned}
$$

A Fig. 5.9, mostra a quantidade

$$
W\left(r, Q^{2}\right)=2 \pi r \int \mathrm{d} z\left(\psi_{\Upsilon}^{*} \psi_{\gamma^{*}}\right)_{T}
$$

que nos dá uma ideia do comportamento da superposição ("overlap") entre a função de onda do fóton virtual e a função de onda do $\Upsilon$ integrada em $z$, em função do tamanho do dipolo $r$. O gráfico foi feito para $Q^{2}=0.05 \mathrm{GeV}^{2}$ e para a parte transversal de $\left(\psi_{\Upsilon}^{*} \psi_{\gamma^{*}}\right.$ ) (já que estamos interessados em fotoprodução) usando os modelos Gaus-LC e Boosted Gaussian para a função de onda do $\Upsilon$. Podemos observar que os dois modelos possuem um pico para pequenos valores de $r$, o que está diretamente associado com a massa do bottom. Ou seja, a função de onda "seleciona" pequenos valores para o tamanho do dipolo. Além disso, 

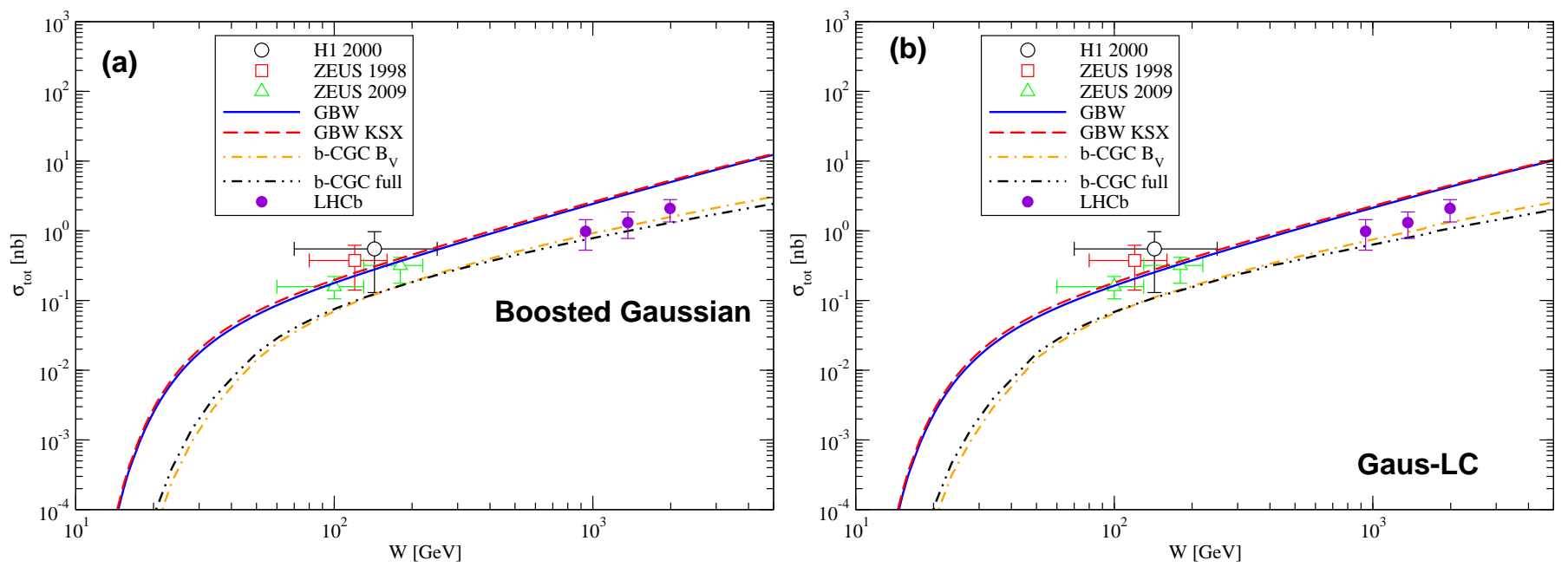

Fig. 5.10: Seção de choque de fotoprodução de $\Upsilon$ em colisões $\gamma p$.

podemos notar que embora tenham formas bastante parecidas, as normalizações para os dois modelos de função de onda são diferentes, levando a diferentes normalizações para as distribuições de rapidez e consequentemente, diferentes valores para as seções de choque.

A fim de estimar seções de choque $\gamma p \rightarrow V p$, é necessário especificar o expoente $\lambda$ para determinar $R_{g}$ e $\beta$. Em geral o fator $\lambda$ é fortemente dependente da distribuição de pártons utilizada. Por outro lado, tal dependência é menor em escalas duras e pequeno $x$. Portanto, devemos esperar que esse seja o caso para a produção de $\Upsilon$ no LHC. Vamos seguir a Ref.[50] e desconsiderar a dependência de $\lambda$ na escala considerando que o mesmo seja dado pelo expoente que determina a dependência da escala de saturação. Além disso, como no caso da produção de $J / \psi$, aqui desconsideramos as interações soft, que destruiriam o gap de rapidez.

Na Fig.5.10 apresentamos nossos resultados para a seção de choque de fotoprodução exclusiva de $\Upsilon$ em colisões $\gamma p$ para os modelos Boosted Gaussian (à esquerda) e GausLC (à direita). Aqui apresentamos dados experimentais de HERA (mais baixa energia) (Refs.[103, 104, 105]) e do LHC (Ref.[106]). É importante ressaltar que nossas predições foram publicadas antes dos dados da Ref.[106]. Como pode ser observado pelos gráficos, os dados experimentais estão dentro da incerteza teórica prevista por nossos cálculos. Além disso, os dados de HERA são melhor descritos pelas duas versões do modelo GBW, enquanto que os dados do LHC são melhor descritos pelas duas versões do modelo bCGC. Uma possível explicação para as diferenças entre as predições de cada modelo está no comportamento de $\mathcal{N}$ para $r$ pequeno (regime linear). Por um lado, temos que $\mathcal{N}_{G B W} \propto r^{2}$, enquanto que $\mathcal{N}_{b C G C} \propto r^{2 \gamma_{e f f}}$ com $\gamma_{\text {eff }} \leq 1$. Além disso, podemos observar que a aproximação da dependência em $t$ para a seção de choque é uma boa aproximação para $W \leq 200 \mathrm{GeV}$, mas superestima a seção de choque para valores maiores da energia de centro de massa $\gamma p$. 

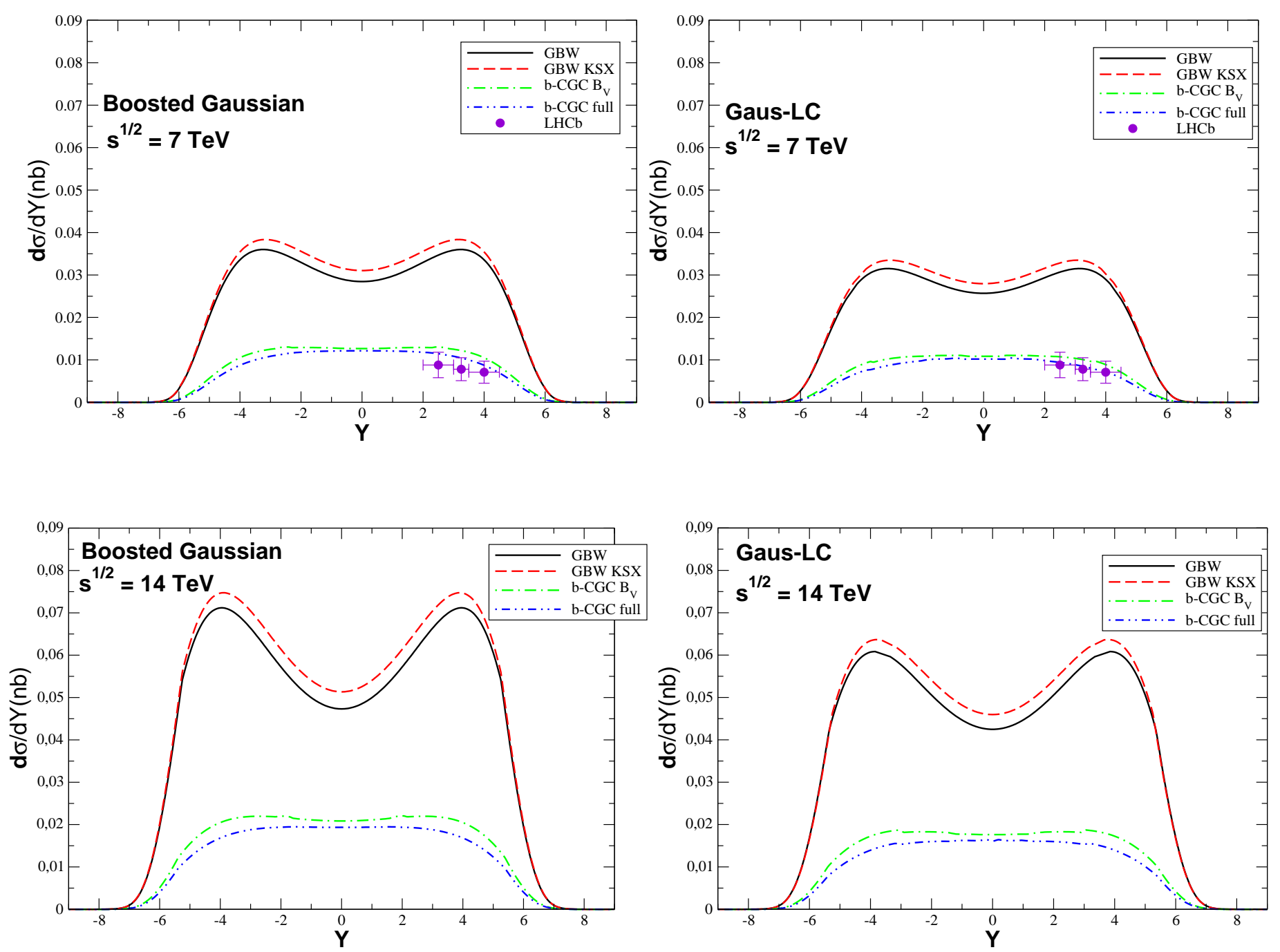

Fig. 5.11: Distribuição de rapidez para a fotoprodução difrativa de $\Upsilon$ em colisões pp a $\sqrt{s}=7 \mathrm{TeV}$ (acima) e a $\sqrt{s}=14 \mathrm{TeV}$ (abaixo). À esquerda usamos o modelo Boosted Gaussian para a função de onda do méson e à direita, o modelo Gaus$L C$.

\subsubsection{Colisões pp e pPb}

Nesta subseção vamos apresentar nossas predições para as seções de choque e distribuições de rapidez para a fotoprodução exclusiva de $\Upsilon$. As equações básicas já foram apresentadas no início da subseção 5.2.2, para o caso da produção de $J / \psi$.

Nos gráficos da Fig. 5.11, mostramos nossos resultados de distribuição de rapidez para a fotoprodução exclusiva de $\Upsilon$ em colisões $p p$ a $\sqrt{s}=7$ (acima) e $14 \mathrm{TeV}$ (abaixo). Conforme esperado, as diferenças entre as predições da seção de choque $\gamma p$ (Fig. 5.10) são refletidas nas distribuições de rapidez para o processo $p p$. Podemos observar que as predições feitas com o 

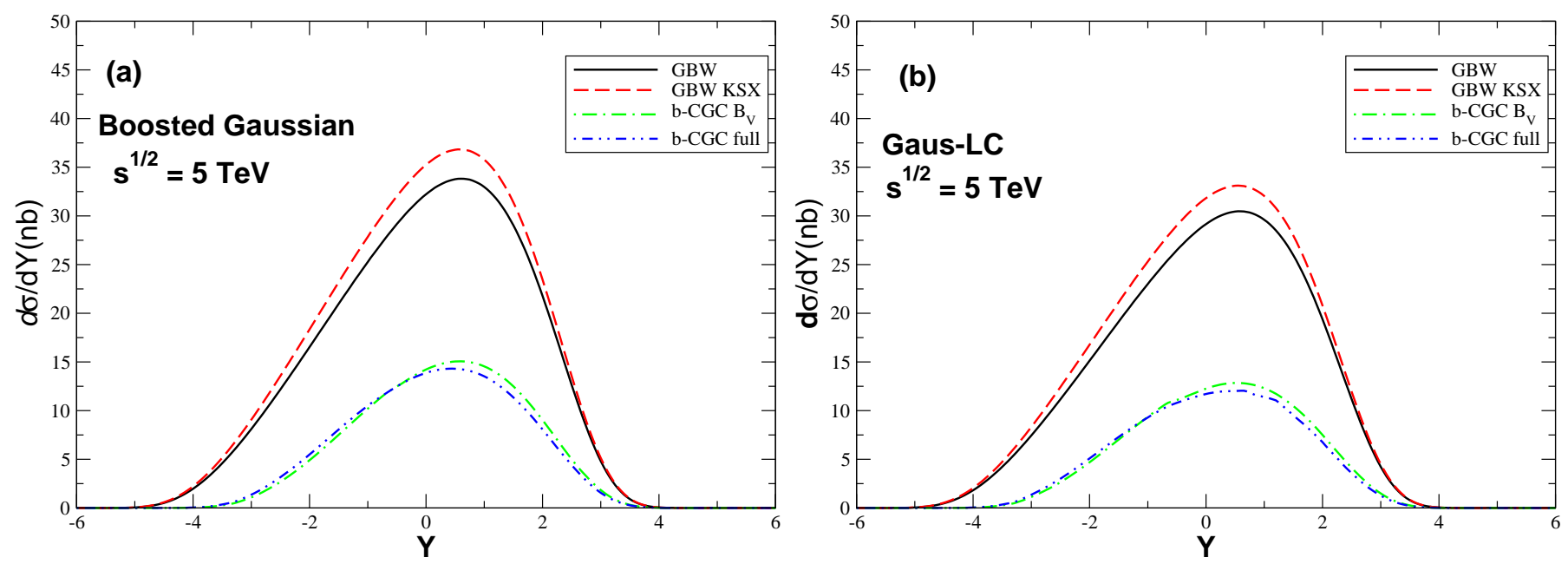

Fig. 5.12: Distribuição de rapidez para a fotoprodução difrativa de $\Upsilon$ em colisões $p P b$ em $\sqrt{s}=5$ TeV. À esquerda usamos o modelo Boosted Gaussian para a função de onda do méson e à direita, o modelo Gaus-LC.

modelo GBW-KSX nos fornecem o limite superior, enquanto que as do modelo bCGC full nos fornecem o limite inferior. As duas predições do modelo bCGC são praticamente idênticas em rapidez central passando a apresentar uma pequena diferença em $|Y| \approx 4$. As predições de GBW KSX e GBW apresentam uma pequena diferença em suas normalizações para o intervalo $|Y| \leq 4$. Além disso, as predições dos modelos GBW e bCGC diferem por um fator $2,7(3,5)$ para $Y=0(4)$, o que está relacionado com a dependência em energia da seção de choque $\gamma p$ para cada modelo, conforme discutido no último parágrafo da seçõ anterior. Para $\sqrt{s}=7 \mathrm{TeV}$ confrontamos nossas predições com os dados do LHCb [106]. Podemos observar que as predições feitas com o modelo bCGC apresentam boa concordância com a experiência. Para $\sqrt{s}=14 \mathrm{TeV}$ obtivemos resultados semelhantes. Contudo as diferenças entre as predições feitas com o GBW e com o bCGC (para as duas versões de cada um) são amplificadas com o aumento da energia. Por fim, como comentado anteriormente, podemos observar uma diferença na normalização das seções de choque dependendo do modelo de função de onda do $\Upsilon$ usado.

A seguir, apresentaremos nossas predições da distribuição de rapidez para a fotoprodução de $\Upsilon$ em colisões $p A$. Cabe aqui um breve comentário sobre esse tipo de interação. Poderíamos nos questionar, em um primeiro momento, se não seria necessário considerar interações do tipo $\gamma \mathrm{Pb}$, caso onde o próton atuaria como fonte de fótons. Como mostrado no capítulo 4, o fluxo de fótons equivalentes e, portanto, a seção de choque de interação $\gamma p$ será aumentada por um fator $Z^{2}\left(Z^{2}=(82)^{2}=6724\right.$ para o chumbo). Dessa forma concluímos que a interação $\gamma P b$ pode ser desprezada quando comparada com a interação $\gamma p$. 


\begin{tabular}{ccccc}
\hline \hline & GBW & GBW KSX & bCGC $B_{V}$ & bCGC full \\
\hline \hline$p p(\sqrt{s}=7 \mathrm{TeV})$ & $298.0 \mathrm{pb}$ & $318.0 \mathrm{pb}$ & $104.0 \mathrm{pb}$ & $91.0 \mathrm{pb}$ \\
$p p(\sqrt{s}=8 \mathrm{TeV})$ & $344.0 \mathrm{pb}$ & $366.0 \mathrm{pb}$ & $118.0 \mathrm{pb}$ & $103.0 \mathrm{pb}$ \\
$p p(\sqrt{s}=14 \mathrm{TeV})$ & $607.0 \mathrm{pb}$ & $638.0 \mathrm{pb}$ & $196.0 \mathrm{pb}$ & $167.0 \mathrm{pb}$ \\
$p \mathrm{~Pb}(\sqrt{s}=5 \mathrm{TeV})$ & $143.8 \mathrm{nb}$ & $157.2 \mathrm{nb}$ & $55.9 \mathrm{nb}$ & $54.4 \mathrm{nb}$ \\
\hline \hline \multicolumn{5}{c}{ Boosted Gaussian } \\
\hline$p p(\sqrt{s}=7 \mathrm{TeV})$ & $340.0 \mathrm{pb}$ & $363.0 \mathrm{pb}$ & $123.0 \mathrm{pb}$ & $110.0 \mathrm{pb}$ \\
$p p(\sqrt{s}=8 \mathrm{TeV})$ & $393.0 \mathrm{pb}$ & $419.0 \mathrm{pb}$ & $140.0 \mathrm{pb}$ & $124.0 \mathrm{pb}$ \\
$p p(\sqrt{s}=14 \mathrm{TeV})$ & $699.0 \mathrm{pb}$ & $740.0 \mathrm{pb}$ & $233.0 \mathrm{pb}$ & $201.0 \mathrm{pb}$ \\
$p \mathrm{~Pb}(\sqrt{s}=5 \mathrm{TeV})$ & $129.9 \mathrm{nb}$ & $141.7 \mathrm{nb}$ & $48.8 \mathrm{nb}$ & $46.9 \mathrm{nb}$ \\
\hline
\end{tabular}

Tab. 5.2: Seções de choque totais de fotoprodução exclusiva de $\Upsilon$ em colisões pp em $\sqrt{s}=$ 7, 8 e $14 \mathrm{TeV}$ e colisões $\mathrm{pPb}$ em $\sqrt{s}=5 \mathrm{TeV}$ considerando os modelos Gaus- $L C$ e Boosted Gaussian para a função de onda do $\Upsilon$.

Na Fig. 5.12, apresentamos nossas predições para a produção de $\Upsilon$ em colisões $p P b$ em $\sqrt{s}=5 \mathrm{TeV}$. Como esperado, a distribuição de rapidez é assimétrica em torno de $Y=0$. Observamos que as predições para GBW diferem por um fator 2.6 das predições de bCGC em $Y=0$. Na Tab. 5.2, apresentamos nossas predições para a seção de choque total de fotoprodução de $\Upsilon$ em colisões $p p$ e $p P b$ em energias do LHC. Como esperado de nossa análise da distribuição de rapidez e do comportamento das seções de choque $\gamma p$ com $W$, as predições para as seções de choque total são fortemente dependentes do modelo de $\mathcal{N}$ utilizado.

\subsection{Previsões para o Run II do LHC}

Nesta seção apresentamos nossas previsões para a produção dos mésons vetoriais $\rho, \phi, J / \psi$ e $\Upsilon$ em colisões $p p$ a $\sqrt{s}=13 \mathrm{TeV}$ e $\mathrm{PbPb}$ (coerentes) em $\sqrt{s}=5.02 \mathrm{TeV}$. O atual interesse desse estudo se deve ao fato de que estas energias serão atingidas no Run II do LHC, cujos primeiros dados já estão sendo divulgados. Em particular, nesse trabalho também estimamos a produção de mésons leves ( $\rho$ e $\phi)$. Aqui apresentaremos, seguindo o formalismo de dipolo (como desenvolvido nas seções anteriores desse capítulo), predições dos modelos bCGC NEW e IIM-RS (versão atualizada do IIM obtida na Ref.[50]) para a amplitude de espalhamento $\mathcal{N}$ e dos modelos Gaus-LC e Boosted Gaussian para a função de onda do méson vetorial. Nosso objetivo é estimar a incerteza teórica de seções de choque e distribuições de rapidez. Esses resultados fazem parte de um artigo que está em preparação.

\subsubsection{Resultados para colisões $p p$ e $P b P b$}

Como comentado no início dessa seção para o processo $p p$ utilizamos o modelo bCGC NEW (como só utilizamos sua versão mais recente, denotaremos aqui apenas por bCGC) 
integrado em $t$, enquanto que para o IIM-RS (a partir daqui denotaremos apenas por IIM) utilizamos o parâmetro de slope $B$ utilizados nas Refs.[107, 108, 109], dado por

$$
B=B_{0}+4 \alpha^{\prime} \ln \left(\frac{W}{W_{0}}\right)
$$

onde $W$ é a energia de centro de massa $\gamma p$. Os valores $B_{0}, \alpha^{\prime}$ e $W_{0}$ são, respectivamente:

- $4.99 \mathrm{GeV}^{-2}, 0.25 \mathrm{GeV}^{-2}$ e $90 \mathrm{GeV}$, para $J / \psi$;

- $3.68 \mathrm{GeV}^{-2}, 0.164 \mathrm{GeV}^{-2}$ e $95 \mathrm{GeV}$, para $\Upsilon$;

- $11 \mathrm{GeV}^{-2}, 0.25 \mathrm{GeV}^{-2}$ e $95 \mathrm{GeV}$, para $\rho$ e $\phi$.

Nossa escolha com relação às versões dos modelos bCGC e IIM se devem ao fato de serem bastante atuais. Para a produção coerente de mésons vetoriais em colisões $P b P b$ utilizamos a Eq.(5.20), a qual independe de $B$.

Vamos inicialmente fazer uma breve discussão a respeito do overlap entre as funções de onda do fóton e do méson vetorial para $Q^{2}=0$ para os dois modelos de $\psi_{V}^{*} \psi_{\gamma}$. Para isso vamos analisar a quantidade $W(r)$ definida na Eq. (5.31). Essa quantidade nos dá o overlap entre as funções de onda do fóton e do méson vetorial $\left(\psi_{V}^{*} \psi_{\gamma}\right)$. Como estamos interessados na fotoprodução $\left(Q^{2} \approx 0\right)$, vamos considerar apenas a polarização transversal para $Q^{2}=0$ (a polarização longitudinal se anula nesse caso). A Fig. 5.13 mostra o comportamento de $W(r)$ em função da separação transversal do dipolo $r$ para todos os mésons considerados em $Q^{2}=0$. Esses gráficos nos mostram que a função $W(r)$ seleciona dipolos "grandes" para mésons leves (Fig. 5.13 à esquerda) e dipolos "pequenos" para mésons pesados (Fig. 5.13 à direita). Por esta razão, os mésons leves são mais sensíveis aos efeitos de saturação do que os mésons pesados, conforme discutido no capítulo 3. Portanto o estudo de estados finais distintos é muito importante, tendo em vista que diferentes massas provam diferentes dinâmicas no limite de altas energias.
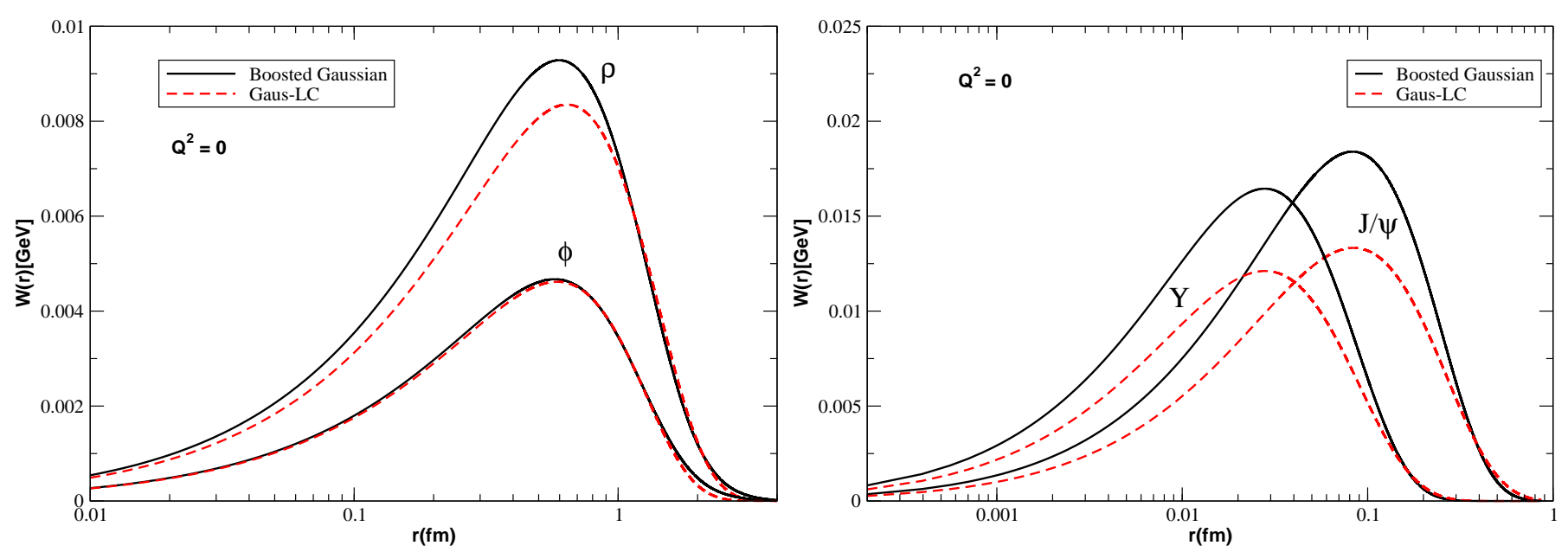

Fig. 5.13: Contribuição transversal do overlap entre as funções de onda do fóton e do méson vetorial integrados em z para $Q^{2}=0$. 

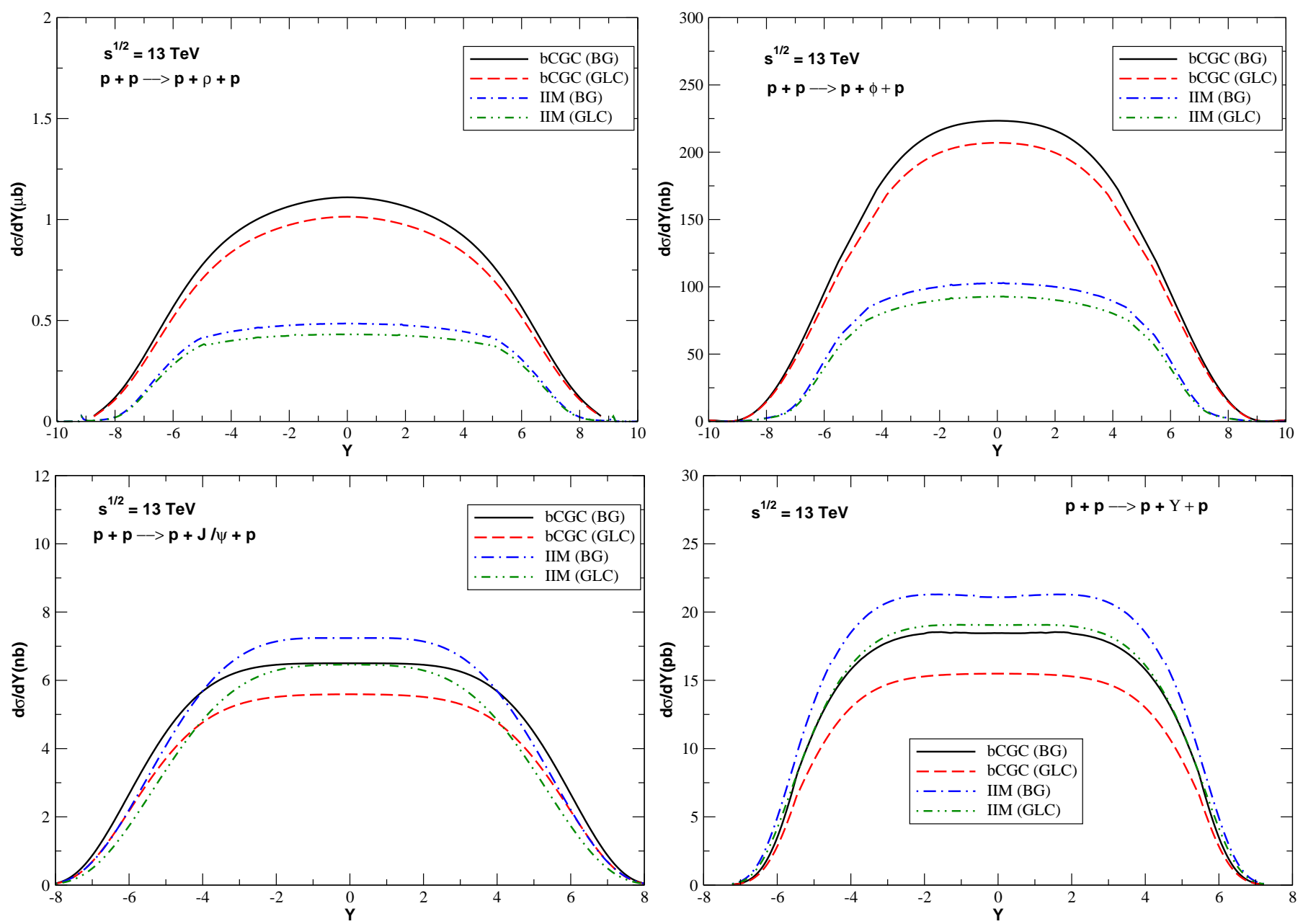

Fig. 5.14: Distribuições de rapidez em colisões pp a $\sqrt{s}=13 \mathrm{TeV}$.

Na Fig.5.14, apresentamos nossas predições para a produção de mésons vetoriais em colisões $p p$ em $\sqrt{s}=13 \mathrm{TeV}$. Podemos observar que, em rapidez central, o modelo bCGC fornece predições maiores do que as feitas com o modelo IIM para mésons leves e menores para mésons pesados. Além disso, as predições para a produção de mésons leves feitas com os modelo bCGC são, aproximadamente, o dobro do que as feitas com o IIM para rapidez central. Em contraste, as predições para a produção de $J / \psi$ e $\Upsilon$ possuem maior concordância entre si. Como já observado nas seções anteriores e esperado pela Fig.5.13, os cálculos feitos com o modelo Boosted Gaussian para $\psi_{V}$ fornecem normalizações superiores do que os feitos com o modelo Gaus-LC, para um mesmo modelo de $\mathcal{N}$.

Nossas predições para a produção de mésons vetoriais em colisões $P b P b$ em $\sqrt{s}=5.02$ TeV são mostradas na Fig.5.15. Conclusões semelhantes ao caso $p p$ podem ser obtidas. A Tab.5.3 contém nossas predições para as seções de choque totais. 

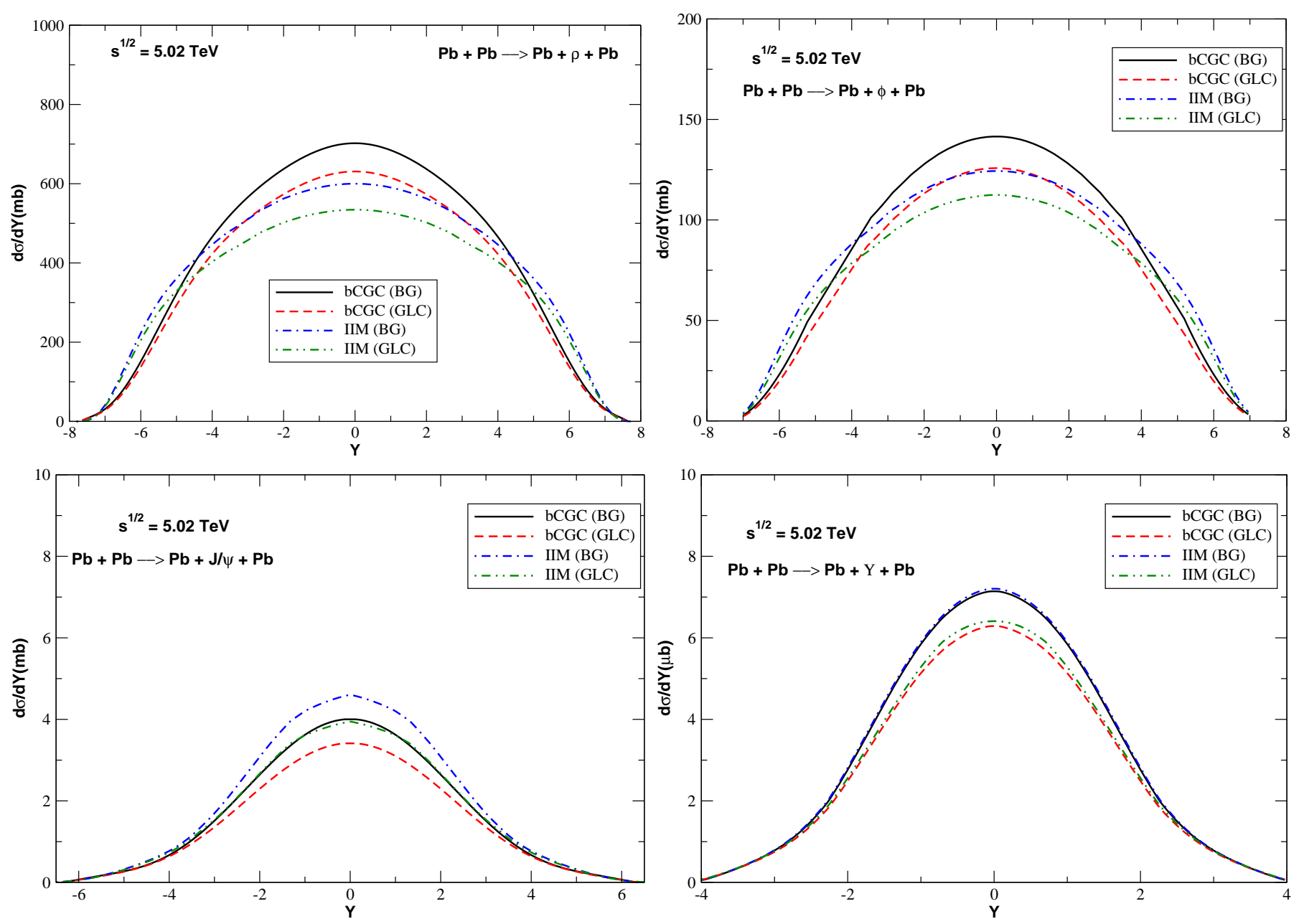

Fig. 5.15: Distribuições de rapidez em colisões $\mathrm{PbPb} a \sqrt{s}=5.02 \mathrm{TeV}$.

\subsection{Conclusões}

Nesse capítulo estudamos a produção de mésons vetoriais em colisões ultraperiféricas utilizando o formalismo de dipolo de cor e a aproximação de fótons equivalentes. Nosso objetivo era estimar a incerteza teórica de alguns dos modelos da física de saturação presentes na literatura. Em particular, nossos resultados estão em boa concordância com os dados experimentais do LHC para a produção de $J / \psi$ e $\Upsilon$. Além disso, estendemos nossos estudos para a produção de mésons vetoriais para energias disponíveis no Run 2 do LHC. Por fim, ressaltamos que esses processos podem ser usados em conjunto com diversos outros onde a física de saturação é aplicável de forma a fornecer um maior conhecimento da dinâmica da QCD em altas energias. 


\begin{tabular}{|ccc|ccc|}
\hline \multicolumn{3}{|c|}{$\mathrm{pp}(\sqrt{s}=13 \mathrm{TeV})$} & \multicolumn{3}{c|}{$\operatorname{PbPb}(\sqrt{s}=5,02 \mathrm{TeV})$} \\
\hline \hline Meson & bCGC & IIM & Meson & bCGC & IIM \\
\hline \hline \multicolumn{3}{|c|}{ Gauss-LC } & & \multicolumn{3}{c|}{ Gauss-LC } \\
$\phi$ & $11.74 \mu \mathrm{b}$ & $5.39 \mu \mathrm{b}$ & $\rho$ & $5794.1 \mathrm{mb}$ & $5454.00 \mathrm{mb}$ \\
$J / \psi$ & $2272.1 \mathrm{nb}$ & $1029.72 \mathrm{nb}$ & $\phi$ & $1083.6 \mathrm{mb}$ & $1069.67 \mathrm{mb}$ \\
$\Upsilon$ & $61.13 \mathrm{nb}$ & $64.48 \mathrm{nb}$ & $J / \psi$ & $18.84 \mathrm{mb}$ & $21.76 \mathrm{mb}$ \\
\hline \hline \multicolumn{3}{|c|}{ Boosted Gaussian } & $195.31 \mathrm{pb}$ & $\Upsilon$ & \multicolumn{3}{c|}{ Boosted Gaussian } \\
\hline$\rho$ & $156.74 \mathrm{pb}$ & $6.00 \mu \mathrm{b}$ & $\rho$ & $6416.5 \mathrm{mb}$ & $6069.15 \mathrm{mb}$ \\
$\phi$ & $2462.7 \mathrm{nb}$ & $1145.96 \mathrm{nb}$ & $\phi$ & $1228.72 \mathrm{mb}$ & $1194.65 \mathrm{mb}$ \\
$J / \psi$ & $72.38 \mathrm{nb}$ & $74.80 \mathrm{nb}$ & $J / \psi$ & $21.58 \mathrm{mb}$ & $24.82 \mathrm{mb}$ \\
$\Upsilon$ & $189.48 \mathrm{pb}$ & $221.68 \mathrm{pb}$ & $\Upsilon$ & $26.02 \mu$ & $26.31 \mu \mathrm{b}$ \\
\hline
\end{tabular}

Tab. 5.3: Seções de choque totais de fotoprodução exclusiva de mésons vetoriais em colisões pp em $\sqrt{s}=13 \mathrm{TeV}$ e colisões $\mathrm{PbPb} \mathrm{em} \sqrt{\mathrm{s}}=5$,02 TeV. 


\section{Capítulo 6}

\section{Produção dupla de mésons vetoriais em colisões $\gamma \gamma$}

Neste capítulo apresentamos nossas previsões para a produção dupla de mésons vetoriais em interações $\gamma \gamma$. Nas primeiras seções apresentamos as principais ferramentas para o estudo de seções de choque para a produção de dois mésons vetoriais $V_{1}$ e $V_{2}$ através da interação fóton-fóton. Conforme será discutido, este estudo pode ser feito com o formalismo de dipolo, onde a interação $\gamma \gamma$ é vista como uma interação dipolo-dipolo. Em seguida apresentamos nossas predições para o futuro colisor $e^{+} e^{-}$chamado ILC ("International Linear Collider"), onde analisamos o comportamento das seções de choque $\gamma-\gamma$ com a energia e com a virtualidade dos fótons, para diferentes modelos das amplitudes de espalhamento dipolo - hádron e dipolo - dipolo. Em seguida mostramos uma possível maneira de estudar a produção dupla de mésons vetoriais em colisores hadrônicos. Para isso, consideramos as seções de choque $\gamma-\gamma$ para $Q^{2}=0$ e utilizamos a generalização da aproximação de fótons equivalentes para o caso do processo fóton-fóton que é induzido inicialmente pelo processo ultraperiférico $p p, p P b$ e $P b P b$. Por fim, apresentamos nossas previsões para seções de choque e distribuições de rapidez para a produção dupla de mésons vetoriais em energias do LHC e do FCC ("Future Circular Collider"). Os resultados discutidos nesse capítulo foram publicados na Ref. [3] (para a produção dupla de mésons vetoriais em colisões $\gamma \gamma$ ) e na Ref. [4] (para a produção dupla de mésons vetoriais em colisões ultraperiféricas hádron-hádron).

\subsection{Formalismo de dipolo para a produção dupla de mésons vetoriais}

Vamos tratar esse tipo de processo considerando que cada fóton virtual flutua em um dipolo de cor, de forma que o processo fóton-fóton ocorre devido à interação dipolo-dipolo. Após o espalhamento dipolo-dipolo os dois mésons vetoriais são formados. Nos nossos cálculos, usamos o formalismo de dipolo considerando diferentes modelos para a amplitude de espalhamento dipolo-próton $\mathcal{N}$ e para a seção de choque dipolo-dipolo $\sigma_{d d}$, enquanto fixamos apenas um modelo para a função de onda do méson vetorial. Vamos estudar a dependência das seções de choque com a energia e com a virtualidade. Como veremos, a investigação do espalhamento fóton-fóton em altas energias pode ser útil para o estudo da 


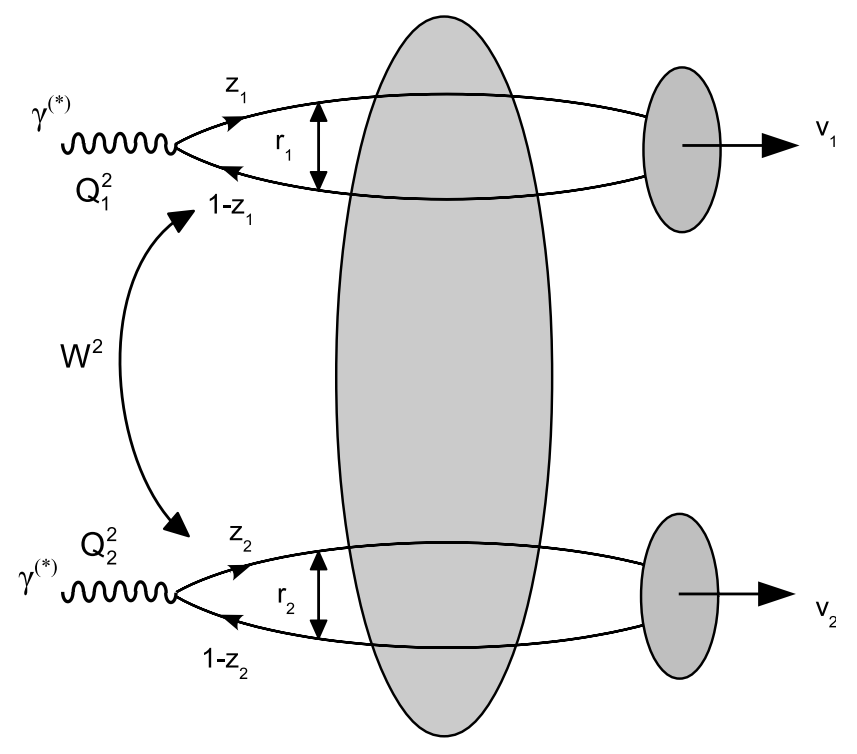

Fig. 6.1: Produção dupla de mésons vetoriais em interações $\gamma^{*} \gamma^{*}$ em altas energias na representação de dipolos de cor.

dinâmica da QCD em pequeno $x$.

Para a amplitude de espalhamento dipolo-próton, usamos os modelos rcBK e IIM-S e para as funções de onda de mésons vetorias o modelo Gaus-LC. A seguir, vamos discutir o formalismo da interação fóton-fóton.

O processo em que estamos interessados é $\gamma^{*} \gamma^{*} \rightarrow V_{1} V_{2}$ onde $V_{i}=\rho, \phi, J / \psi, \Upsilon$. Em altas energias, este espalhamento pode ser estudado com o formalismo de dipolo de cor. A Fig. 6.1 mostra dois fótons virtuais que flutuam em pares $q \bar{q}$ que interagem entre si dando origem a dois mésons vetoriais no estado final. A energia de centro de massa ao quadrado no processo $\gamma^{*} \gamma^{*}$ é dada por $W^{2}=(p+q)^{2}$, sendo $p$ e $q$ os momentos dos fótons. Podemos ainda definir as virtualidades dos fótons como $Q_{1}^{2}=-q^{2}$ e $Q_{2}^{2}=-p^{2}$. Além disso, $t$ é o quadrado do momento transferido. Desta forma, a seção de choque total de produção dupla de mésons vetoriais é $[110,111]$

$$
\begin{aligned}
\sigma\left(\gamma \gamma \rightarrow V_{1} V_{2}\right) & =\int \mathrm{d} t \frac{\mathrm{d} \sigma\left(\gamma \gamma \rightarrow V_{1} V_{2}\right)}{\mathrm{d} t} \\
& =\left.\frac{1}{B_{V_{1} V_{2}}} \frac{\mathrm{d} \sigma\left(\gamma \gamma \rightarrow V_{1} V_{2}\right)}{\mathrm{d} t}\right|_{t=0} \\
& =\frac{|\operatorname{Im} \mathcal{A}(s, t=0)|^{2}}{16 \pi B_{V_{1} V_{2}}}
\end{aligned}
$$


onde $\operatorname{Im} \mathcal{A}(s, t=0)$ é dada por

$$
\begin{aligned}
\operatorname{Im} \mathcal{A}\left(\gamma^{*} \gamma^{*} \rightarrow V_{1} V_{2}\right) & =\sum_{h, \bar{h}} \sum_{n, \bar{n}} \int \mathrm{d} z_{1} \mathrm{~d}^{2} r_{1} \Psi_{h, \bar{h}}^{\gamma}\left(z, r_{1}, Q_{1}^{2}\right) \Psi_{h, \bar{h}}^{V_{1} *}\left(z_{1}, r_{1}\right) \\
& \times \int \mathrm{d} z_{2} \mathrm{~d}^{2} r_{2} \Psi_{n, \bar{n}}^{\gamma}\left(z_{2}, r_{2}, Q_{2}^{2}\right) \Psi_{n, \bar{n}}^{V_{2} *}\left(z_{2}, r_{2}\right) \sigma_{d d}\left(x_{12}, r_{1}, r_{2}\right),
\end{aligned}
$$

sendo $\Psi^{\gamma}\left(\Psi^{V_{i}}\right)$ a função de onda do fóton virtual (méson vetorial). As helicidades dos quarks e antiquarks são denotadas por $h, \bar{h}, n$ e $\bar{n}$ de forma que as helicidades dos fótons e mésons devam ser implicitamente entendidas. A variável $r_{i}$ é a separação transversal do dipolo $i$ e $z_{i}\left(1-z_{i}\right)$ é a fração do momento do fóton carregada pelo quark $i$ (antiquark $i$ ). A variável $x_{12}$ ( $x$ de Bjorken) é definida de forma diferente para cada modelo de $\sigma_{d d}$.

\subsubsection{Previsões para o ILC}

A seção de choque dipolo-dipolo pode ser escrita como

$$
\sigma^{d d}\left(r_{1}, r_{2}, Y\right)=2 \int \mathrm{d}^{2} b \mathcal{N}^{d d}\left(r_{1}, r_{2}, b, Y\right)
$$

onde $\mathcal{N}^{d d}\left(r_{1}, r_{2}, b, Y\right)$ é a amplitude de espalhamento entre dois dipolos com tamanhos transversais $r_{1}$ e $r_{2}$, parâmetro de impacto relativo $b$ e separação de rapidez $Y$. Vamos usar dois modelos para $\sigma^{d d}$, os quais serão discutidos a seguir.

Na Ref. [112], foi assumido que o parâmetro de impacto relativo entre os dipolos deveria ser $b<R$, onde $R=\operatorname{Max}\left(r_{1}, r_{2}\right)$, de forma que $\mathcal{N}^{d d}$ fosse pequena quando não houvesse sobreposição entre os dipolos $(b>R)$. Desta forma, a seção de choque dipolo-dipolo pode ser escrita como

$$
\sigma^{d d}\left(r_{1}, r_{2}, Y\right)=2 \mathcal{N}(r, Y) \int_{0}^{R} \mathrm{~d}^{2} b=2 \pi R^{2} \mathcal{N}(r, Y)
$$

onde $\mathcal{N}$ é a amplitude de espalhamento frontal dipolo-alvo. A seção de choque $\sigma^{d d}$ pode ser escrita como

$$
\sigma^{d d}\left(r_{1}, r_{2}, Y\right)=2 \pi r_{1}^{2} \mathcal{N}\left(r_{2}, Y_{2}\right) \Theta\left(r_{1}-r_{2}\right)+2 \pi r_{2}^{2} \mathcal{N}\left(r_{1}, Y_{1}\right) \Theta\left(r_{2}-r_{1}\right)
$$

onde $Y_{i}=\ln 1 / x_{i} \mathrm{e}$

$$
x_{i}=\frac{Q_{i}^{2}+4 m_{f}^{2}}{W^{2}+Q_{i}^{2}} .
$$

Neste trabalho, vamos também seguir a Ref. [110] e usar a $\sigma^{d d}$ dada por

$$
\sigma_{a, b}^{d d}\left(r_{1}, r_{2}, Y\right)=\sigma_{0}^{a, b} \mathcal{N}\left(r_{1}, r_{2}, Y\right), \sigma_{0}^{a, b}=\frac{2}{3} \sigma_{0}
$$

onde $\sigma_{0}$ é um parâmetro livre para cada modelo de $\mathcal{N}$. Na equação acima, $\mathcal{N}\left(r_{1}, r_{2}, Y\right)=$ $\mathcal{N}\left(r_{e f f}, Y=\ln \left(1 / \tilde{x}_{a b}\right)\right)$, com

$$
r_{e f f}^{2}=\frac{r_{1}^{2} r_{2}^{2}}{r_{1}^{2}+r_{2}^{2}} \text { e } \tilde{x}_{a b}=\frac{Q_{1}^{2}+Q_{2}^{2}+4 m_{a}^{2}+4 m_{b}^{2}}{W^{2}+Q_{1}^{2}+Q_{2}^{2}} .
$$


Para $\mathcal{N}$ usamos os modelos IIM-S e rcBK. Além disso, nos resultados que serão apresentados a seguir, denotamos a Eq.(6.7) por "Model 1" e a Eq.(6.5) por "Model 2". É importante ressaltar que esses dois modelos para a interação dipolo-dipolo descrevem os dados de LEP ("Large Electron Positron Collider") para as seções de choque totais $\gamma \gamma$ (conforme as Refs.[110, 112]).

Como o valor do slope $B_{V_{1} V_{2}}$ não é bem conhecido para todos os casos, na maioria dos nossos resultados, calculamos o produto $B_{V_{1} V_{2}} \sigma\left(\gamma^{*} \gamma^{*} \rightarrow V_{1} V_{2}\right)$.

Na Fig. 6.2 apresentamos nossos resultados para o comportamento com a energia das seções de choque de produção de dois mésons iguais no estado final com $Q_{1}^{2}=Q_{2}^{2}=$ $Q^{2}=0$. Uma característica marcante do formalismo de dipolo é que ele nos permite tratar simultaneamente a produção dupla de $\rho$ via interação fóton-fóton, que é um processo tipicamente "soft", e a produção dupla de $\Upsilon$ que é um exemplo de processo "hard". Além
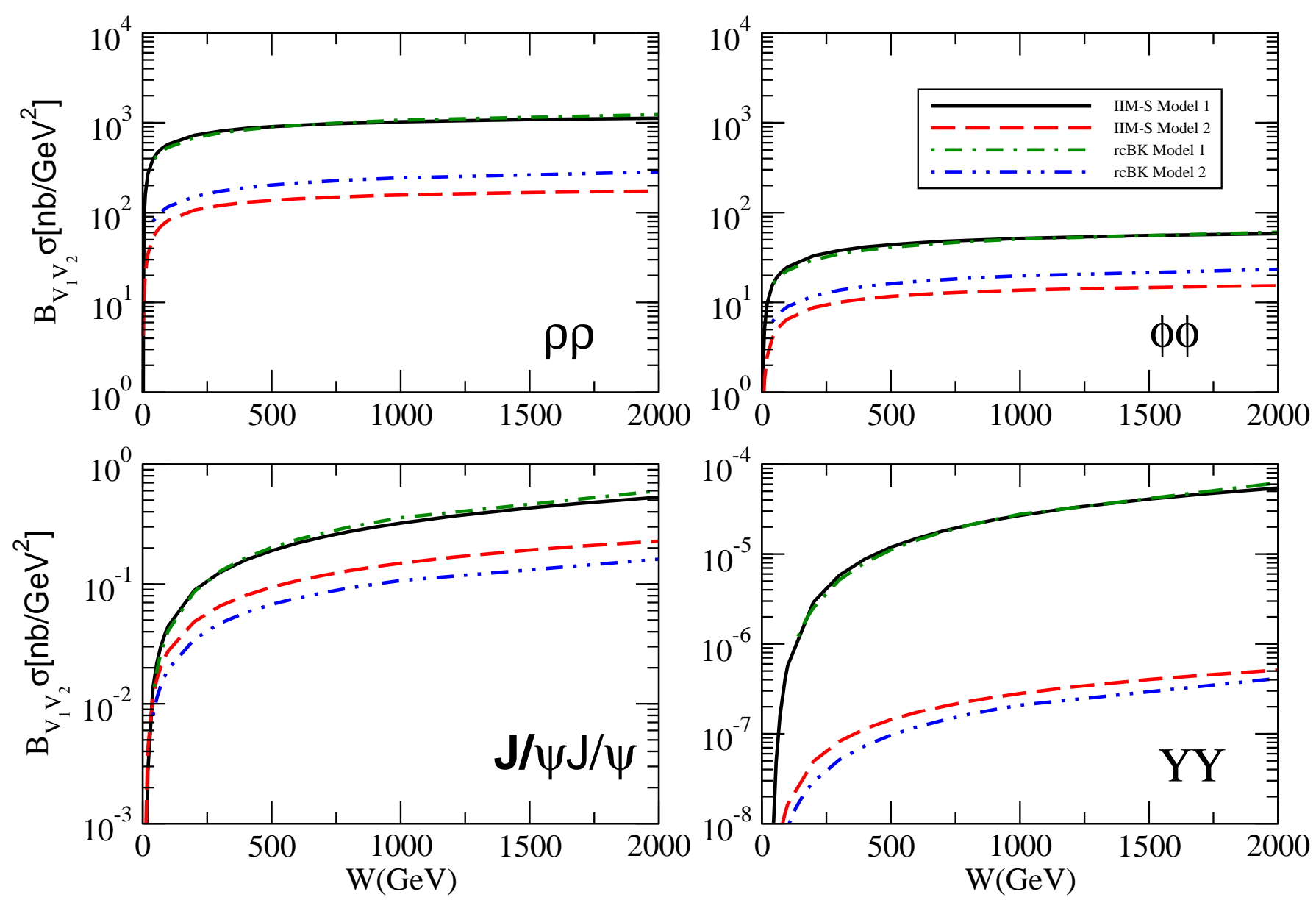

Fig. 6.2: Dependência do produto $B_{V_{1} V_{2}} \sigma\left(\gamma^{*} \gamma^{*} \rightarrow V_{1} V_{2}\right)$ com a energia assumindo $V_{1}=V_{2}$ e considerando $Q_{1}^{2}=Q_{2}^{2}=0$. 

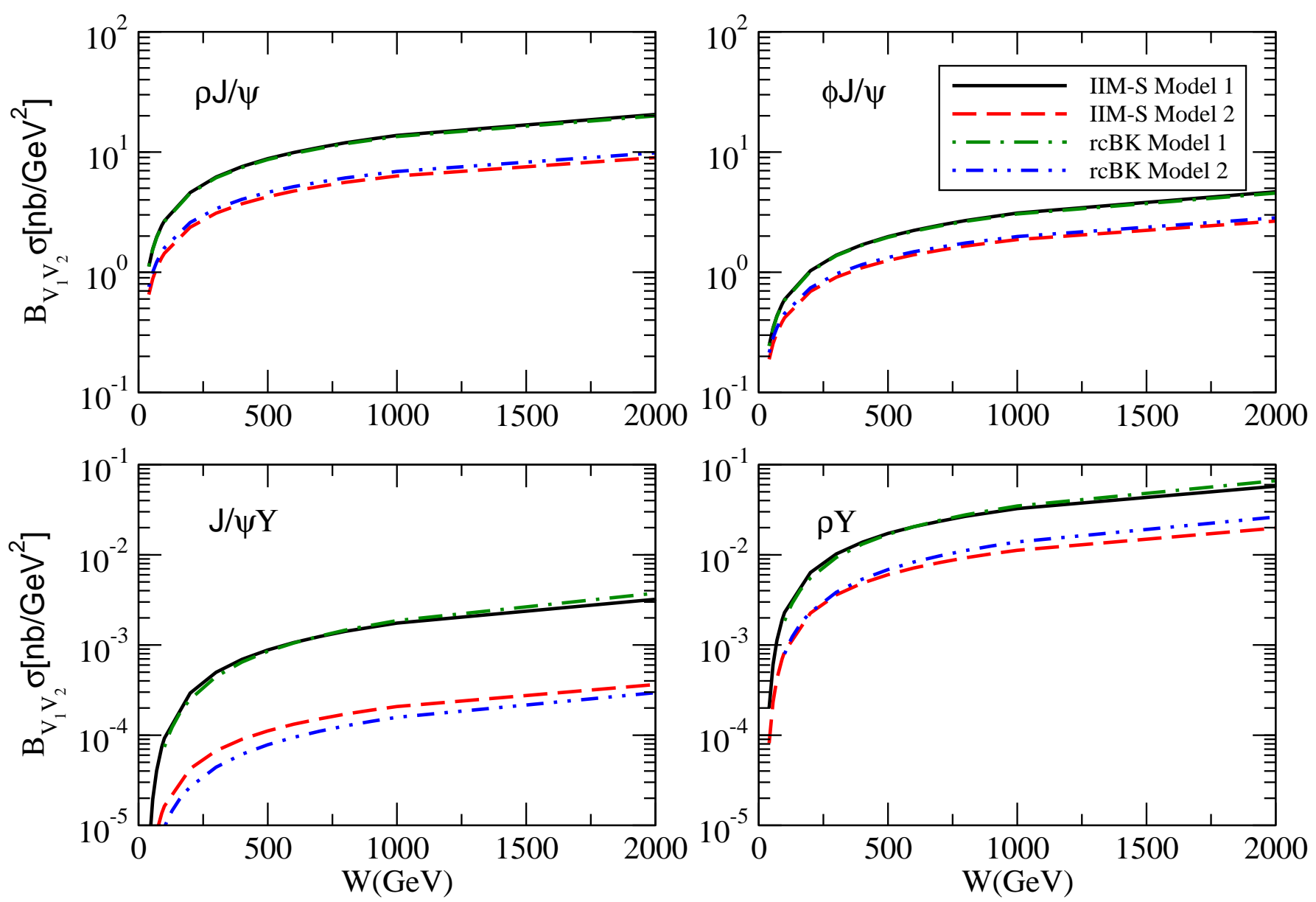

Fig. 6.3: Dependência do produto $B_{V_{1} V_{2}} \sigma\left(\gamma^{*} \gamma^{*} \rightarrow V_{1} V_{2}\right)$ com a energia assumindo $V_{1} \neq V_{2}$ e considerando $Q_{1}^{2}=Q_{2}^{2}=0$.

disso, o estudo da produção dupla de mésons vetoriais via interações fóton-fóton nos permite estudar a transição entre estes dois regimes. Podemos observar que as principais diferenças de previsões ocorrem entre os modelos para o tratamento de $\sigma^{d d}$, sendo as previsões feitas com o modelo 2 sempre menores do que as feitas com o modelo 1. Observamos também que as diferenças entre as previsões feitas com os modelos 1 e 2 (de $\sigma^{d d}$ ) para a produção dupla de $\Upsilon$, que está associada à interação de dois dipolos muito pequenos, são muito grandes quando comparadas com as outras combinações de mésons. Estas diferenças aparecem porque cada modelo de $\sigma_{d d}$ depende da separação dos dipolos e de $x$ de uma maneira diferente. Podemos notar ainda que com o modelo 1 , as previsões feitas com IIM-S e rcBK são praticamente idênticas. Já com o modelo 2 as previsões do modelo IIM-S são menores do que as feitas 

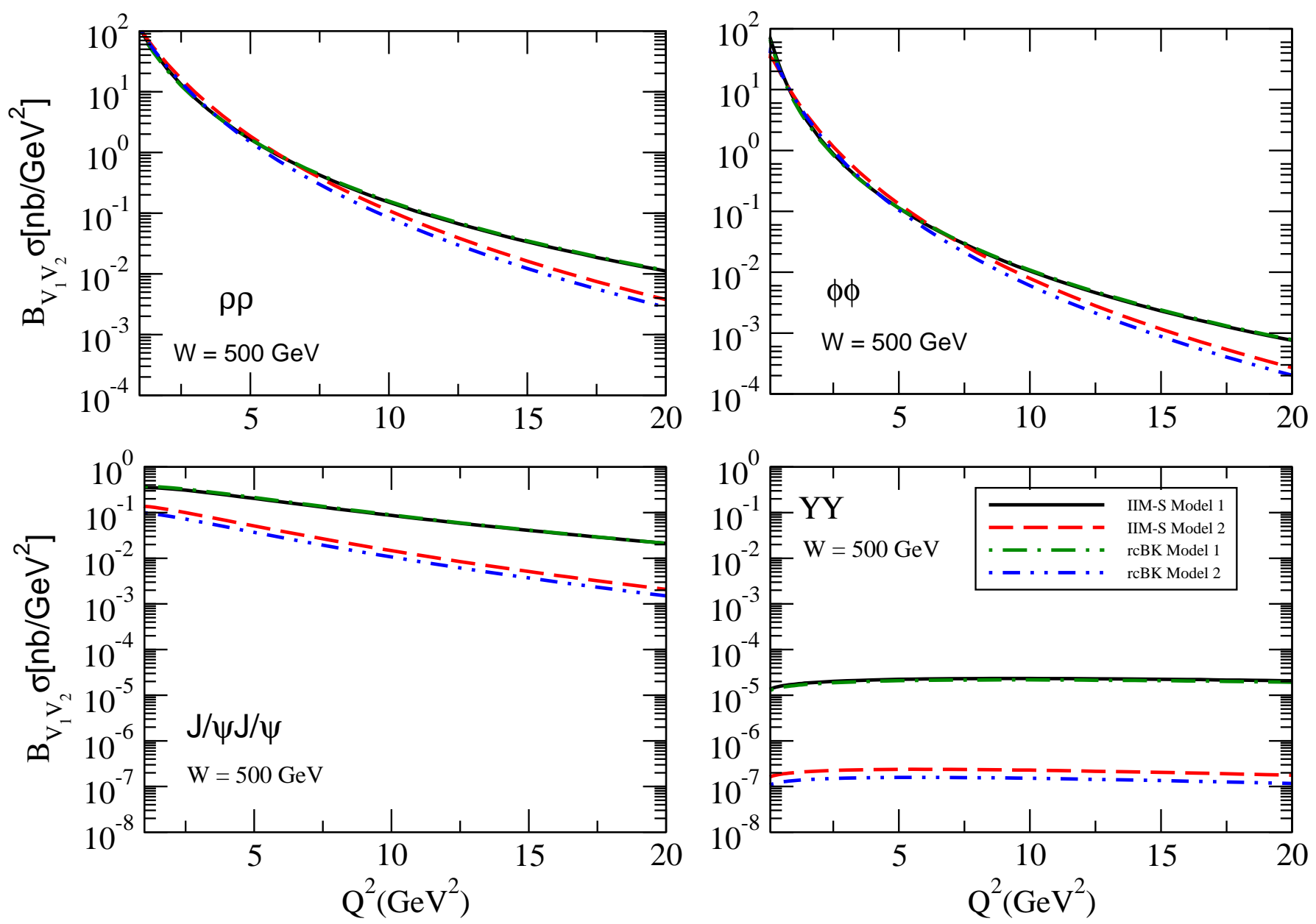

Fig. 6.4: Dependência do produto $B_{V_{1} V_{2}} \sigma\left(\gamma^{*} \gamma^{*} \rightarrow V_{1} V_{2}\right)$ com a virtualidade assumindo $V_{1}=V_{2}$ e considerando $Q_{1}^{2}=Q_{2}^{2}=Q^{2}$ para $W=500 \mathrm{GeV}$.

com o modelo rcBK para combinações de mésons leves e maiores para mésons pesados. Tal comportamento está diretamente associado à forma com a qual a transição entre pequenos e grandes dipolos ocorre para cada modelo de $\mathcal{N}$. Podemos observadar este comportamento também na Fig. 6.3, na qual apresentamos previsões para a produção de dois mésons diferentes no estado final.

A Fig. 6.4 mostra o comportamento das seções de choque de produção de dois mésons iguais no estado final com a virtualidade $\left(Q_{1}^{2}=Q_{2}^{2}=Q^{2}\right)$ para $W=500 \mathrm{GeV}$. Nesse caso devemos levar em conta tanto contribuições transversais quanto longitudinais para as seções de choque. Aqui, temos duas escalas duras ("hard"): $Q^{2}$ e a massa do quark. Na produção de $\rho \rho$ e $\phi \phi$ (mésons leves) a escala dominante é $Q^{2}$ e observamos que as seções de choque caem rapidamente com $Q^{2}$. Na produção de $\Upsilon \Upsilon$ as previsões são praticamente independentes de $Q^{2}$, uma vez que no intervalo considerado a escala que define o tamanho dos dipolos é a 

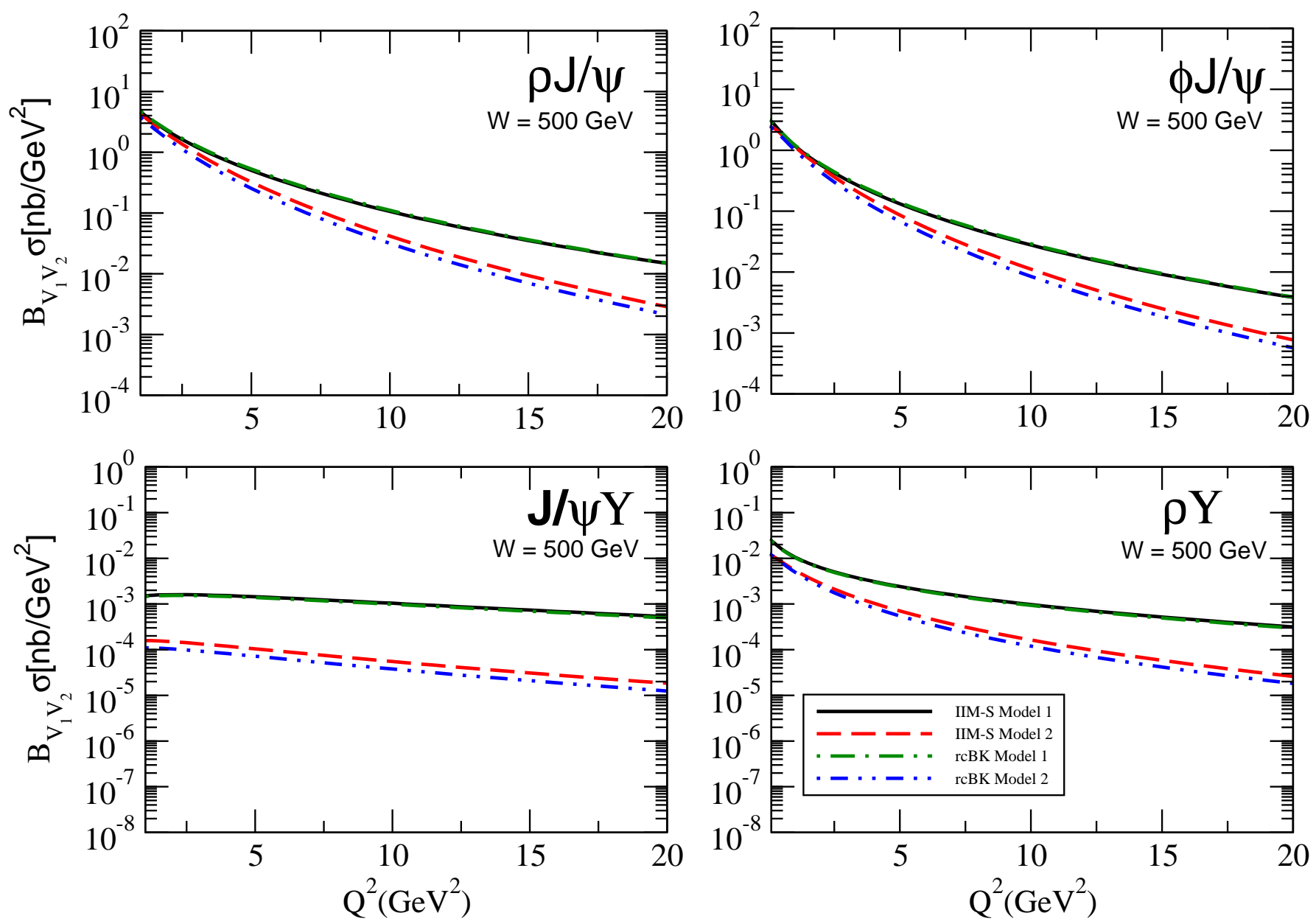

Fig. 6.5: Dependência do produto $B_{V_{1} V_{2}} \sigma\left(\gamma^{*} \gamma^{*} \rightarrow V_{1} V_{2}\right)$ com a virtualidade assumindo $V_{1} \neq V_{2}$ e considerando $Q_{1}^{2}=Q_{2}^{2}=Q^{2}$ para $W=500 \mathrm{GeV}$.

massa do quark bottom. Por outro lado, na produção de $J / \psi J / \psi$, o tamanho típico dos dipolos é dominado pela massa do quark charm para pequeno $Q^{2}$ e pela virtualidade do fóton quando $Q^{2}$ aumenta, levando a uma dependência suave com $Q^{2}$. Além disso, observamos que a diferença entre as previsões feitas com os modelos 1 e 2 para $\sigma^{d d}$ aumenta com o aumento de $Q^{2}$. A Fig. 6.5 mostra resultados semelhantes para a produção de dois mésons diferentes no estado final para $W=500 \mathrm{GeV}$. Nestes gráficos, observamos que as diferenças entre as previsões dos modelos 1 e 2 na região de alto $Q^{2}(\approx 20) \mathrm{GeV}^{2}$ tendem a aumentar com a diminuição das separações transversais dos dipolos.

A Fig. 6.6, mostra o comportamento das seções de choque normalizadas com a energia, virtualidade e massa dos quarks que compõem os mésons vetoriais. As diferentes seções de choque foram normalizadas a $1 \mathrm{em} W=100 \mathrm{GeV}$. Para $Q^{2}=0$ observamos que o crescimento das seções de choque dos mésons pesados é maior com o aumento da energia. 

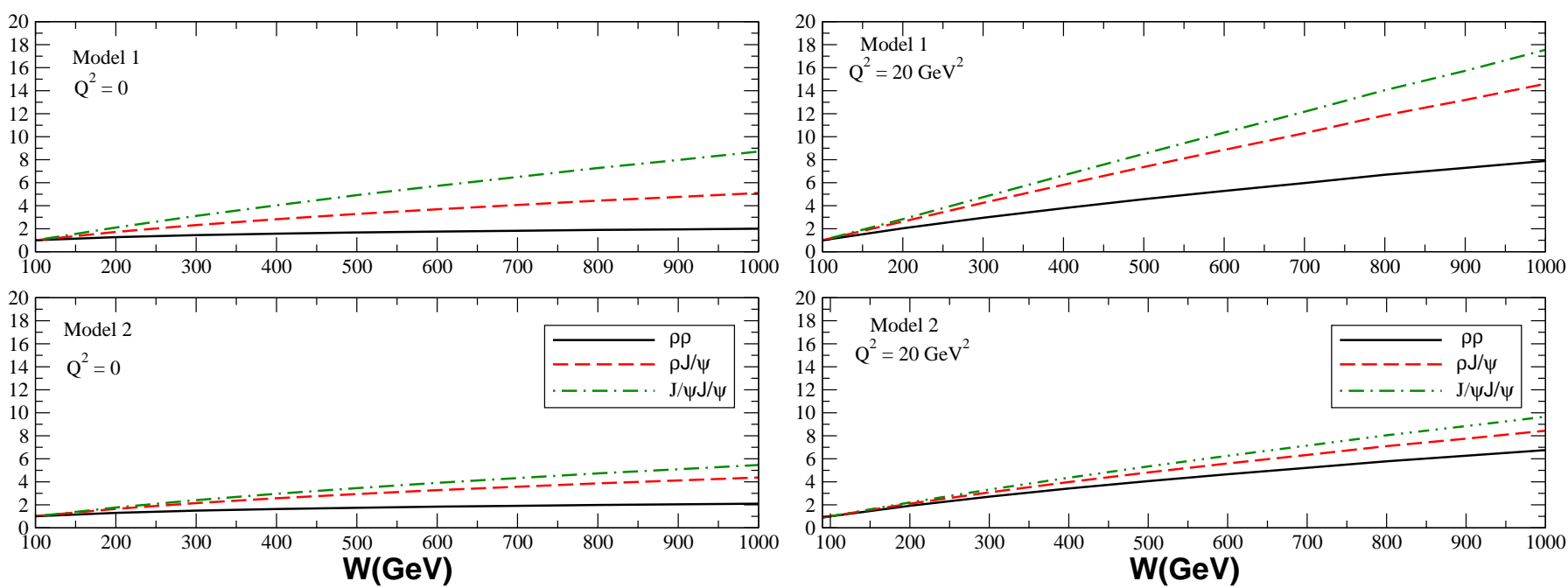

Fig. 6.6: Dependência da seção de choque normalizada com a energia e com a virtualidade $\left(Q_{1}^{2}=Q_{2}^{2}=Q^{2}\right)$.
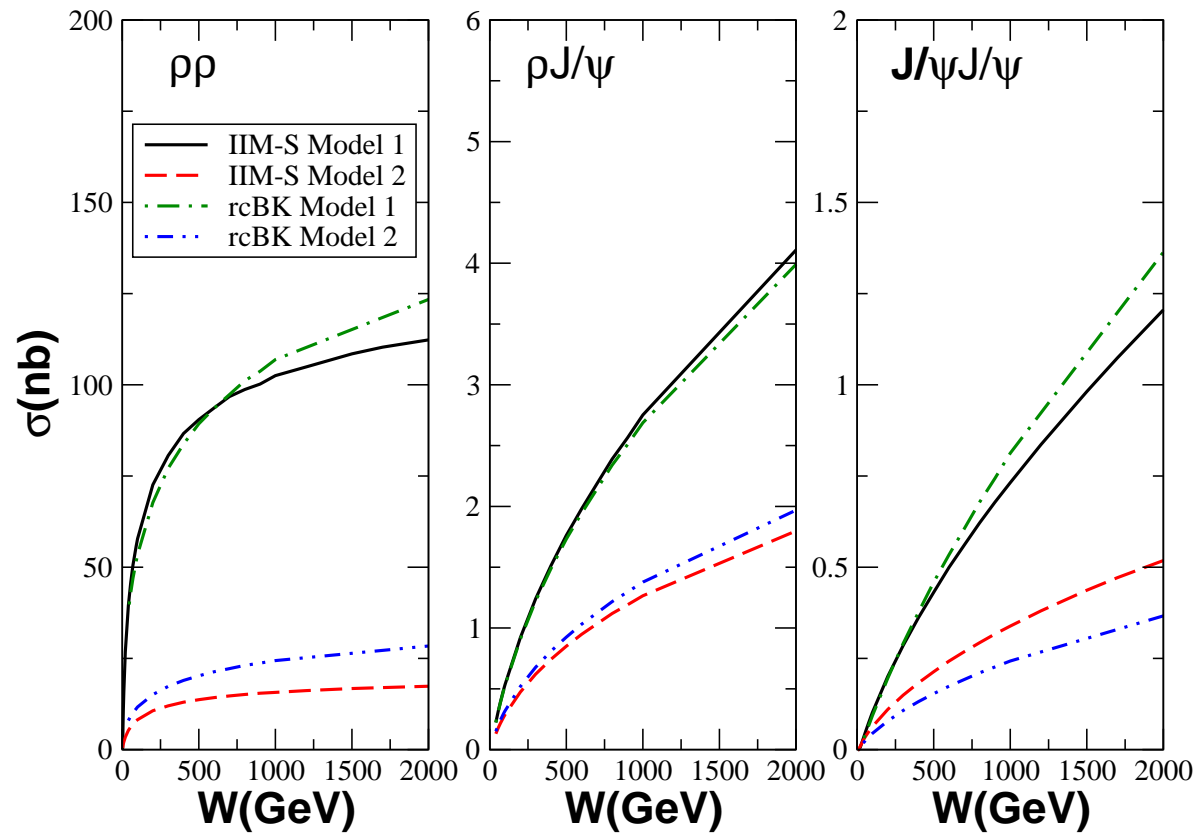

Fig. 6.7: Dependência de $\sigma\left(\gamma^{*} \gamma^{*} \rightarrow V_{1} V_{2}\right)$ com a energia para diferentes estados finais, considerando $Q_{1}^{2}=Q_{2}^{2}=0$. 


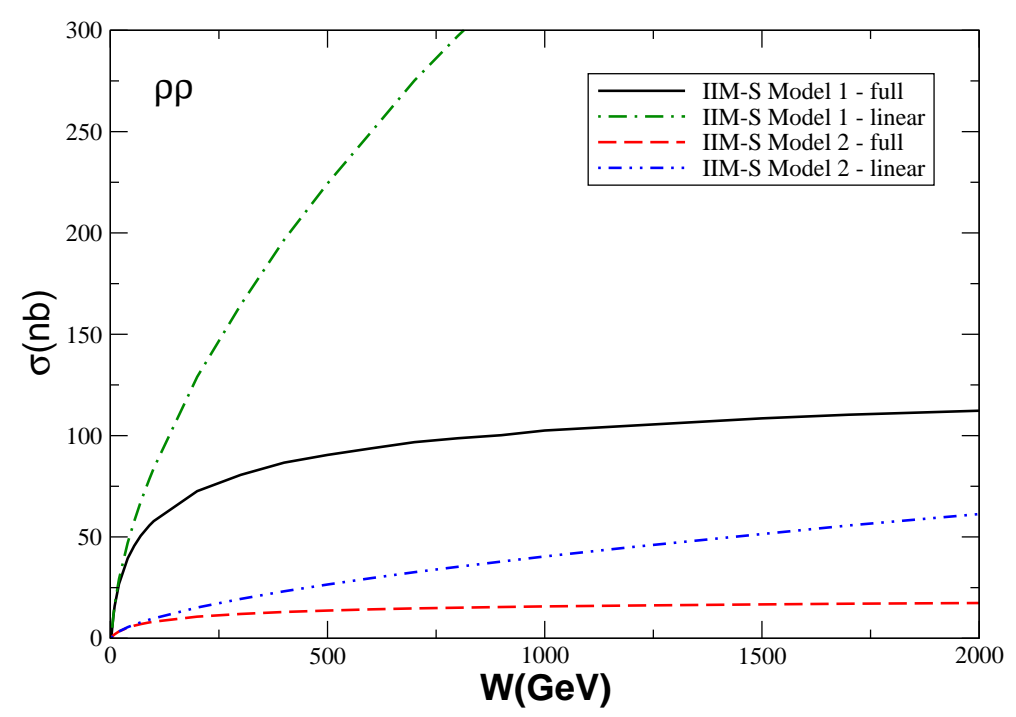

Fig. 6.8: Comparação entre as partes linear e completa do modelo IIM-S para o comportamento da seção de choque $\gamma \gamma \rightarrow \rho \rho$.

Além disso, o modelo 2 para $\sigma^{d d}$ prediz uma inclinação menor do que o modelo 1 . Para $Q^{2}=$ $20 \mathrm{GeV}^{2}$ observamos um comportamento semelhante. Entretanto, neste caso a produção de $\rho \rho$ apresenta um crescimento acentuado com a energia, que está diretamente associado à existência de um $Q^{2} \neq 0$ que funciona como uma escala hard para o processo.

A Fig. 6.7 mostra nossas previsões para a seção de choque de produção de $\rho \rho, \rho J / \psi$ e $J / \psi J / \psi$. Neste caso, diferentemente do que foi feito nas Figs. 6.2, 6.3, 6.4 e 6.5 (onde apresentamos o comportamento do produto $B_{V_{1} V_{2}} \sigma$ com a energia e com a virtualidade), aqui usamos valores de slope extraídos da Ref. [111]: $B_{\rho \rho}=10 \mathrm{GeV}^{-2}, B_{\rho J / \psi}=5 \mathrm{GeV}^{-2}$ e $B_{J / \psi J / \psi}=0,44 \mathrm{GeV}^{-2}$.

Finalmente, na Fig.6.8 apresentamos nossos resultados para a seção de choque de produção de $\rho \rho$ como função da energia para as versões lineares (aproximação de dipolos pequenos) e completa do modelo IIM-S. Podemos observar que o comportamento da seção de choque é fortemente modificado pelos efeitos de saturação.

\subsection{Previsões para o LHC e o FCC}

Nas seções anteriores, apresentamos nossas previsões para a produção dupla de mésons vetoriais em colisões fóton-fóton e concluímos que um estudo experimental deste processo será factível no futuro colisor ILC. Por outro lado, é possível estudar a produção dupla de mésons vetoriais em colisores hadrônicos como o LHC. O diagrama da Fig.6.9, mostra esse processo. Nesta seção discutiremos como este estudo teórico pode ser feito.

A produção dupla de mésons vetoriais em colisões hadrônicas ultraperiféricas devido à interação $\gamma-\gamma$ já foi estudada em vários trabalhos. Na Ref.[113], este estudo foi feito 


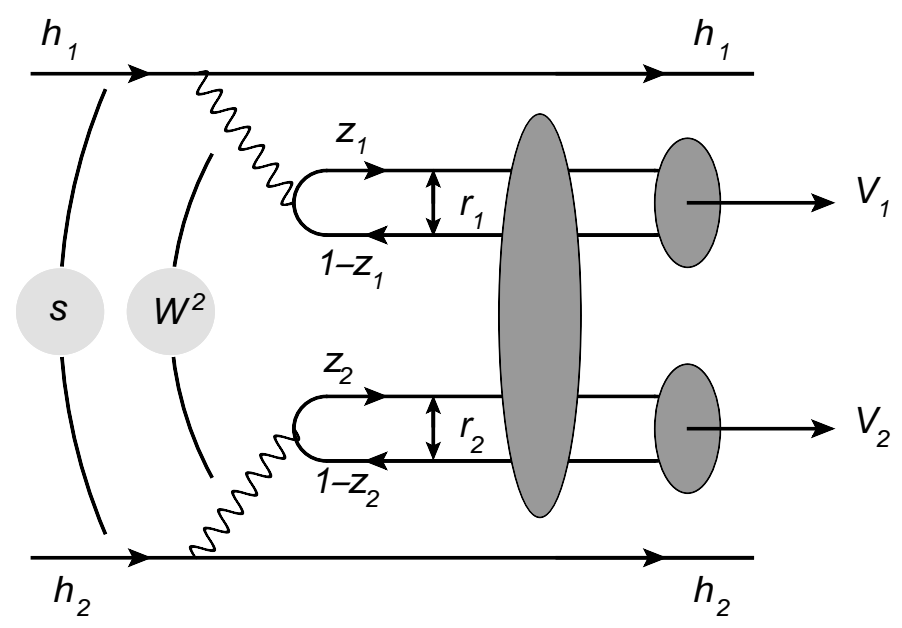

Fig. 6.9: Diagrama para o processo $A A \rightarrow A V V A$ devido à interação $\gamma \gamma$.

considerando-se a troca de dois glúons, que corresponde a mais baixa ordem em QCD em altas energias para o processo. Nas Refs.[114, 115], o cálculo foi feito considerando a dinâmica BFKL. Na Ref.[111], esse processo foi estudado com o formalismo de dipolo, incluindo modelos de saturação. Mais recentemente, nas Refs.[116] e [117] as produções duplas de $\rho$ e $J / \psi$ foram estudadas levando em conta não apenas efeitos de altas energias, mas também efeitos de baixas energias. Nesses trabalhos, foi encontrado que os efeitos de baixas energias são importantes para o processo. Em [116] os efeitos de baixas energias para a produção dupla de $\rho$ foram obtidos por um fit de dados experimentais e os de altas energias através da aproximação VDM-Regge (onde VDM denota o modelo de dominância de mésons vetoriais [18]). Já na Ref.[117], os efeitos de baixas energias foram calculados de diagramas do tipo "caixa" através das regras da QCD e de um formalismo não relativístico para estados ligados, enquanto que os de altas energias são levados em conta pela troca de dois glúons usando fatores de impacto relativístivos (essas contribuições são independentes da energia). Aqui, seguimos as Refs.[116, 117], para tratar a parte de baixas energias e nossos resultados das primeiras seções deste capítulo para altas energias, nos casos onde as virtualidades são iguais a zero. Nossos resultados foram publicados na Ref. [4].

$\mathrm{Na}$ aproximação de fótons equivalentes, a seção de choque de produção de dois mésons vetoriais numa colisão ultrperiférica entre dois hádrons é dada por

$$
\sigma_{h_{1} h_{2}}(\sqrt{s})=\int \sigma_{\gamma \gamma}(W) N\left(\omega_{1}, \vec{b}_{1}\right) N\left(\omega_{2}, \vec{b}_{2}\right) S_{a b s}^{2}(\vec{b}) \mathrm{d}^{2} \vec{b}_{1} \mathrm{~d}^{2} \vec{b}_{2} \mathrm{~d} \omega_{1} \mathrm{~d} \omega_{2}
$$

onde $\sqrt{s}$ é a energia do centro de massa da colisão $h_{1} h_{2}\left(h_{i}=p, P b\right)$ e $W=\sqrt{4 \omega_{1} \omega_{2}}$ é a energia do centro de massa $\gamma \gamma$. Além disso, $N(\omega, b)$ é o espectro de fótons equivalentes para fótons com energia $\omega$ em uma distância $\vec{b}$ da trajetória da fonte (este fluxo é discutido em 
mais detalhes no apêndice C). Este fluxo pode ser escrito em termos do fator de forma da fonte como

$$
N(\omega, b)=\frac{Z^{2} \alpha}{\pi^{2} \omega b^{2}}\left|\int_{0}^{\infty} \mathrm{d} u u^{2} J_{1}(u) \frac{F\left[\sqrt{\frac{u^{2}+\left(\frac{b \omega}{\gamma}\right)^{2}}{b^{2}}}\right]}{\left[u^{2}+\left(\frac{b \omega}{\gamma}\right)^{2}\right]}\right|^{2}
$$

O fator $S_{a b s}^{2}(\vec{b})$ é o fator de absorção dado por

$$
S_{a b s}^{2}(\vec{b})=\Theta\left(|\vec{b}|-R_{1}-R_{2}\right)=\Theta\left(\left|\vec{b}_{1}-\vec{b}_{2}\right|-R_{1}-R_{2}\right)
$$

sendo $R_{1,2}$ os raios das fontes dos fótons. A Eq.(6.9) pode ser escrita de uma forma bastante conveniente para calcularmos distribuições de rapidez. A rapidez do par é definida por

$$
Y=\frac{1}{2}\left(y_{V_{1}}+y_{V_{2}}\right)
$$

e se relaciona com $\omega_{1,2}$ e $W$ da seguinte maneira

$$
\omega_{1}=\frac{W}{2} e^{Y} \text { e } \omega_{2}=\frac{W}{2} e^{-Y}
$$

Assim, a seção de choque para o processo $h_{1} h_{2}$ pode ser escrita como

$$
\sigma_{h_{1} h_{2}}(\sqrt{s})=\int \sigma_{\gamma \gamma}(W) N\left(\omega_{1}, \vec{b}_{1}\right) N\left(\omega_{2}, \vec{b}_{2}\right) S_{a b s}^{2}(\vec{b}) \mathrm{d}^{2} \vec{b}_{1} \mathrm{~d}^{2} \vec{b}_{2} \frac{W}{2} \mathrm{~d} W \mathrm{~d} Y
$$

A fim de estimar seções de choque e distribuições de rapidez da forma mais realista possível, é necessário obter a seção de choque $\sigma_{\gamma \gamma}(W)$ para um grande intervalo de energia. Para altas energias, vamos usar nossas predições feitas com o formalismo de dipolo para a produção dupla de $\rho$ e $J / \psi$, apresentadas na Fig. 6.7, utilizando o modelo 2 para a interação dipolo-dipolo e o modelo IIM-S para $\mathcal{N}$. O formalismo de dipolo não é adequado para baixas energias. Para a produção dupla de $\rho$ e $J / \psi$ em baixas energias, seguiremos as Refs. $[116,117]$. Na Ref. [116], a produção dupla de $\rho$ em baixas energias foi diretamente extraída de dados experimentais de baixa energia. Já na Ref. [117], foi calculada a produção dupla de $J / \psi$ em baixa energia em uma aproximação não relativística. Nessas duas referências, os autores afirmam que as seções de choque $P b P b$ são dominadas por mecanismos de baixas energias. Nosso objetivo neste trabalho é estimar a magnitude dos mecanismos de altas energias em colisões $P b P b$ e apresentar predições para $p p$ e $p P b$. No que segue, apresentaremos nossas predições para as distribuições de rapidez e seções de choque totais denotando por "low energy" (LE) as predições feitas apenas com mecanismos de baixas energias e "low energy + IIM-S" (LHE - low and high energy) para as predições incluindo mecanismos de altas energias.

Na Fig. 6.10 apresentamos nossas predições para a produção dupla de mésons vetoriais em colisões $\mathrm{PbPb}$. Podemos obsrvar que as predições com LE e LHE são quase idênticas na produção dupla de $\rho$ em todas as energias consideradas (em concordância com a Ref. 

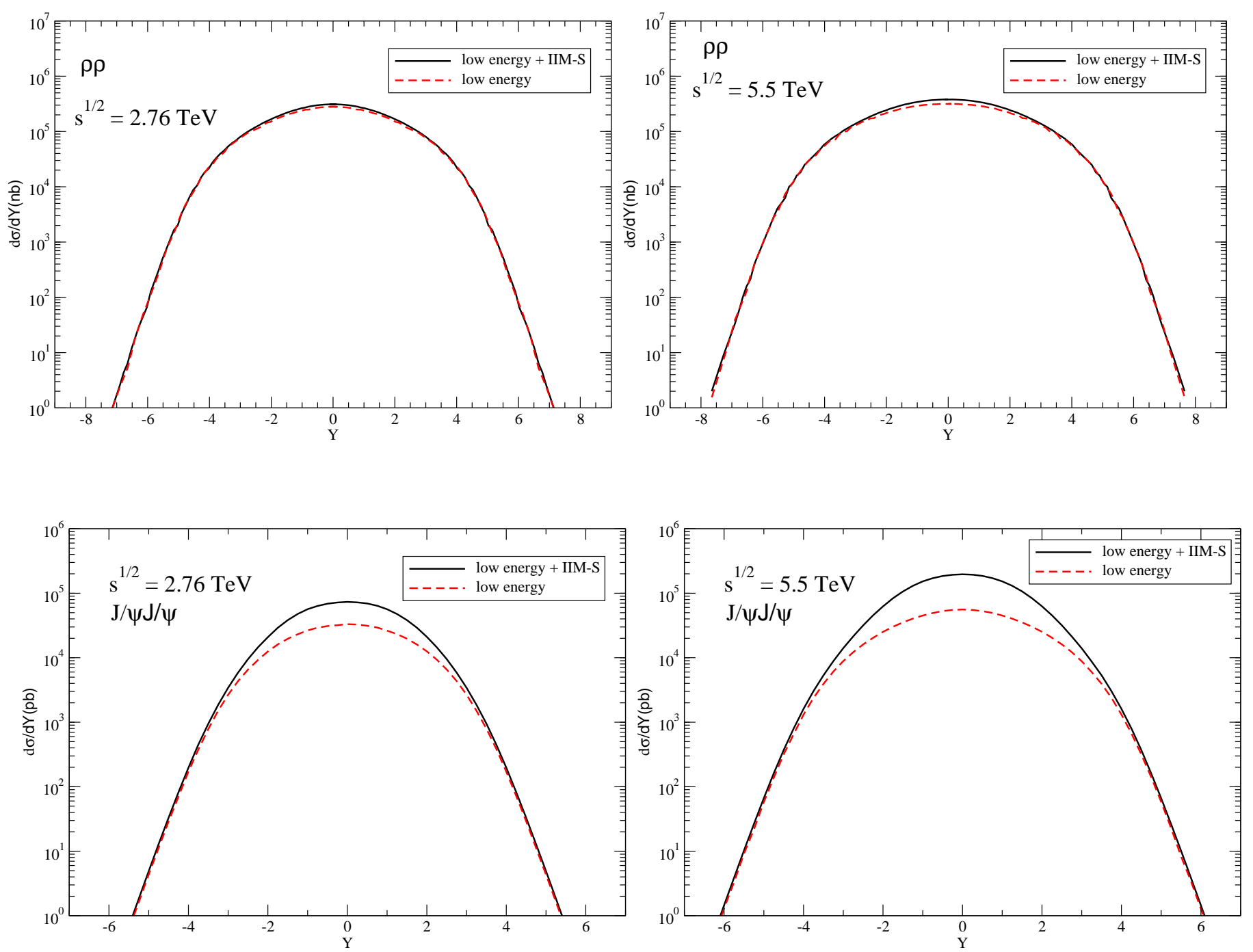

Fig. 6.10: Distribuições de rapidez para a produção dupla de mésons vetoriais em colisões $\mathrm{PbPb}$.

[116]). Este resultado é consequência do comportamento da seção de choque $\gamma \gamma \rightarrow \rho \rho$ a altas energias, que apresenta um crescimento suave com $W$, típico de grandes dipolos. Por outro lado, na produção dupla de $J / \psi$ esperamos que a seção de choque $\gamma \gamma \rightarrow J / \psi J / \psi$ cresça mais rapidamente com $W$. Em particular, observamos que um aumento na energia leva a um aumento da diferença entre as predições LE e LHE na região de rapidez central.

Na Fig. 6.11 apresentamos nossas predições para a produção dupla de mésons vetoriais em colisões $p P b$ em energias do LHC. Como esperado, neste caso a distribuição de rapidez é assimétrica em torno de $Y=0$. Observamos que as diferenças entre as predições LE e LHE começam a se tornar visíveis no caso da produção dupla de $\rho$ e são significativas para a produção dupla de $J / \psi$. A ampliação das diferenças entre as predições LE e LHE em colisões $p P b$, em comparação com $P b P b$ estão associadas à energia máxima $W_{\text {max }}^{\gamma \gamma}$ que 

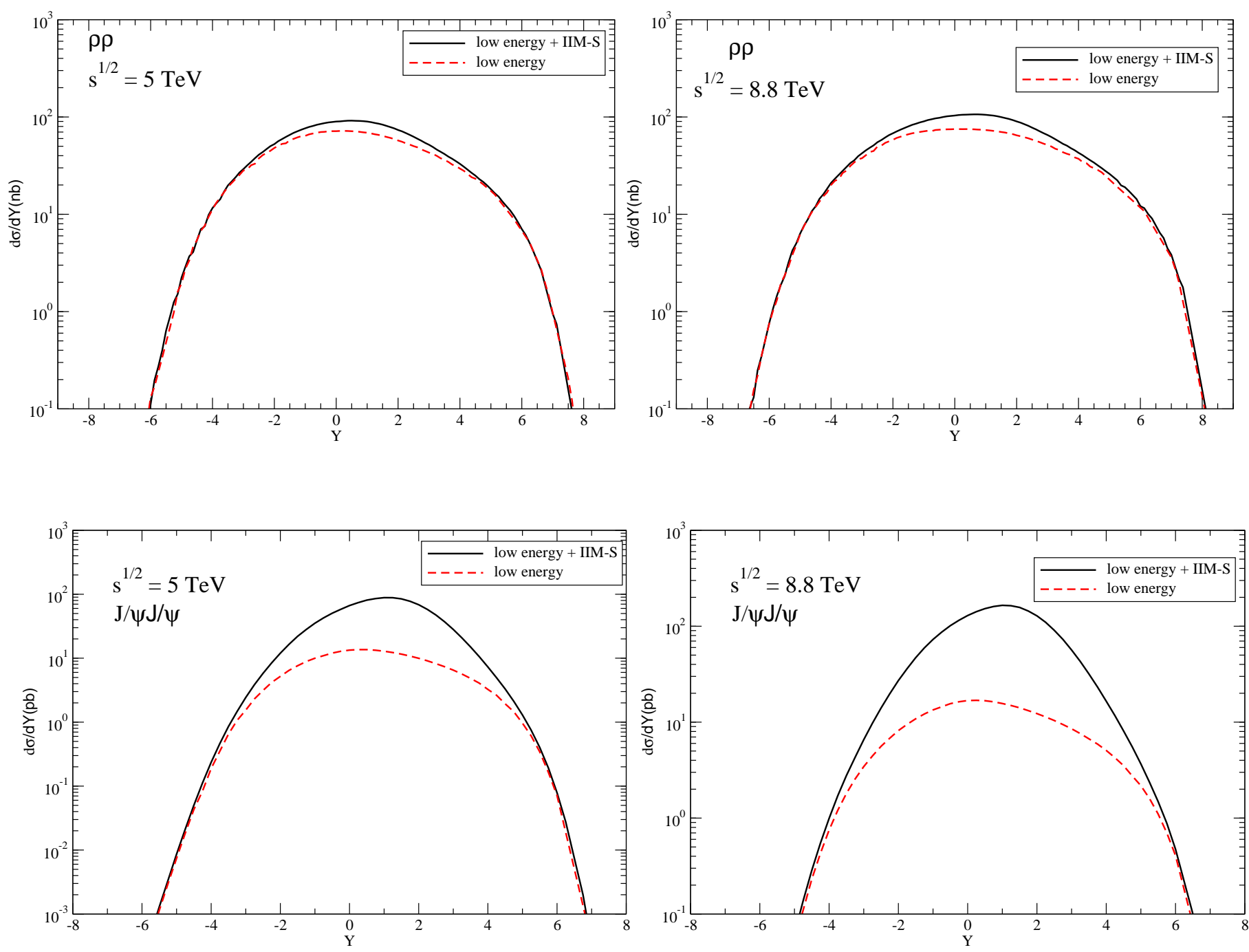

Fig. 6.11: Distribuições de rapidez para a produção dupla de mésons vetoriais em colisões $p P b$.

é atingida em cada colisão. Para o caso $p P b$ em $\sqrt{s}=8.8 \mathrm{TeV}$, temos que $W_{\text {max }}^{\gamma \gamma} \approx 260$ $\mathrm{GeV}$, enquanto que no caso $\mathrm{PbPb}, W_{\text {max }}^{\gamma \gamma} \approx 160 \mathrm{GeV}$. Desta forma, os mecanismos de altas energias possuem mais influência no caso $p P b$ do que no caso $P b P b$.

Na Fig.6.12, são apresentadas nossas predições para a produção dupla de mésons vetoriais em colisões $p p$. Neste caso, para colisões a $\sqrt{s}=14 \mathrm{TeV}$, temos que $W_{\max }^{\gamma \gamma} \approx 4500 \mathrm{GeV}$. Isto implica que a contribuição gluônica associada às altas energias se torna ainda mais importante neste caso. Podemos observar nos gráficos que as diferenças das predições LE e LHE aumentam neste tipo de colisão.

Outra forma de estimar as contribuições gluônicas é através da análise do comportamento das seções de choque dos processos $h_{1} h_{2} \rightarrow h_{1} V V h_{2}$ com a energia do centro de massa $h h$. Tal análise é apresentada na Fig. 6.13. Em concordância com nossas predições para as 

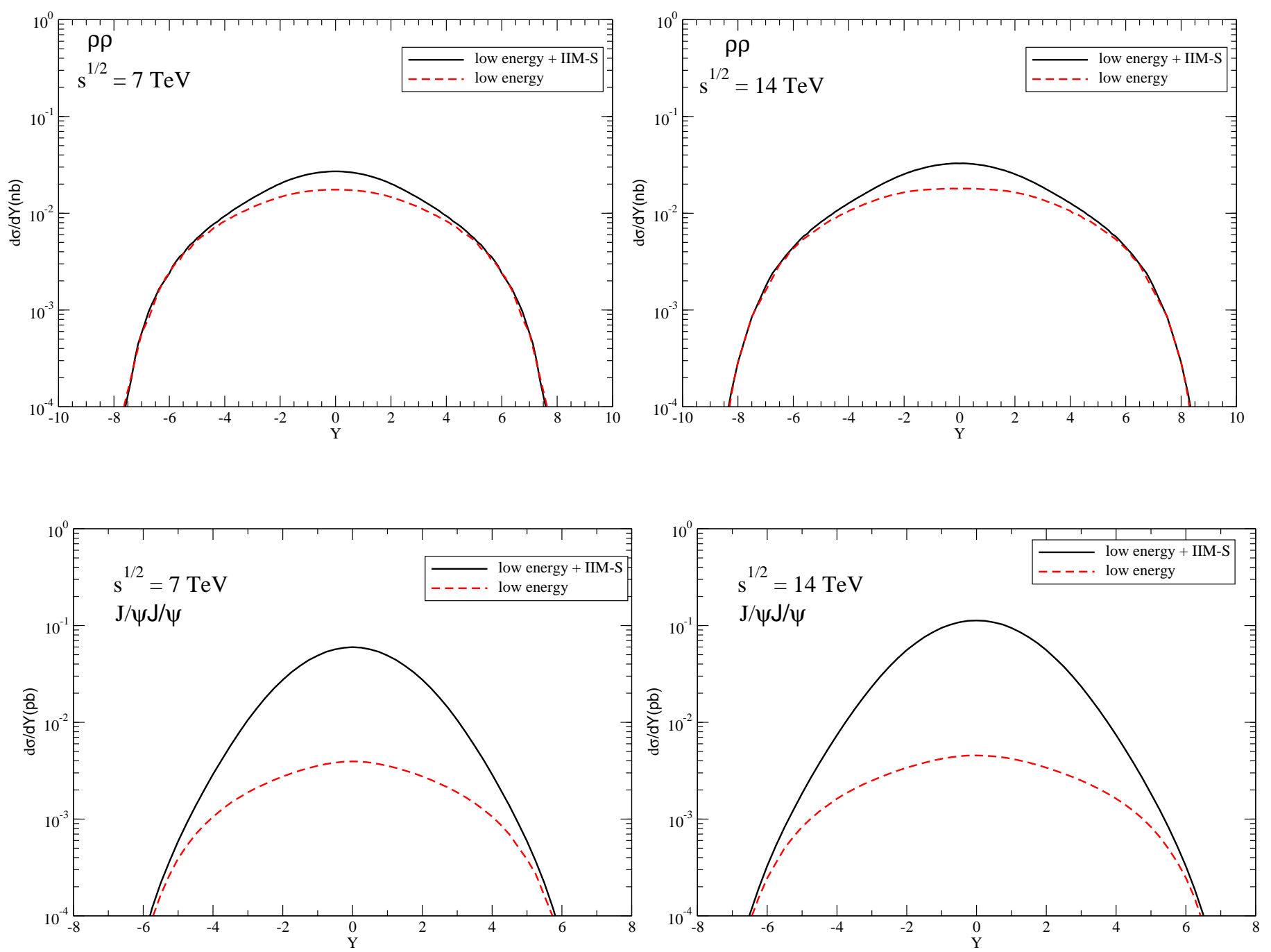

Fig. 6.12: Distribuições de rapidez na produção dupla de mésons vetoriais em colisões pp.

distribuições de rapidez, observamos que a contribuição gluônica é pequena para a produção dupla de $\rho$ e considerável para a produção dupla de $J / \psi$.

Por fim, nas Tabs. 6.1 e 6.2 apresentamos nossas predições para as seções de choque de produção dupla de mésons vetoriais para energias cobertas por vários colisores hadrônicos. Essa tabela nos permite chegar a conclusões similares às conclusões fornecidas pelos gráficos discutidos até aqui. Observamos que as diferenças entre as predições LE e LHE para duplo $\rho$ são bem menores do que as do caso da produção dupla de $J / \psi$. Observamos também que conforme aumentamos a energia de centro de massa da colisão, os efeitos de altas energias começam a ficar cada vez maiores. Em particular, destacamos que as seções de choque estudadas em intervalos de energia de futuros colisores como o FCC e CEPC - SPPC $\left(\sqrt{s}_{A A}=39 \mathrm{TeV}, \sqrt{s}_{p A}=63 \mathrm{TeV}\right.$ e $\left.\sqrt{s}_{p p}=100 \mathrm{TeV}\right)$ fornecem diferenças bastante razoáveis entre as predições com os mecanismos LE e LHE para a produção dupla de $J / \psi$. Portanto 

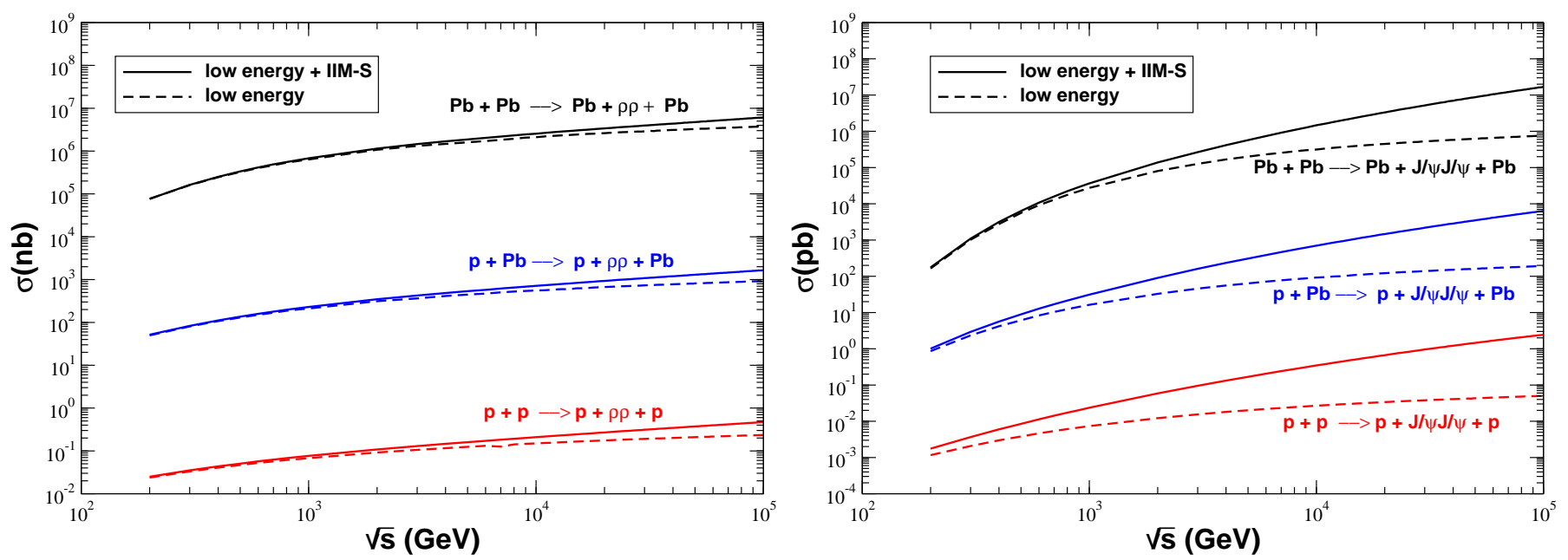

Fig. 6.13: Comportamento das seções de choque com $\sqrt{s}$.

\begin{tabular}{lll}
\hline \hline & Low energy & Low energy + IIM-S \\
\hline \hline$P b P b(\sqrt{s}=500 \mathrm{GeV})$ & $0.33 \times 10^{6}$ & $0.33 \times 10^{6}$ \\
$\mathrm{PbPb}(\sqrt{s}=2.76 \mathrm{TeV})$ & $1.27 \times 10^{6}$ & $1.39 \times 10^{6}$ \\
$\mathrm{PbPb}(\sqrt{s}=5.5 \mathrm{TeV})$ & $1.73 \times 10^{6}$ & $1.97 \times 10^{6}$ \\
$\mathrm{PbPb}(\sqrt{s}=39 \mathrm{TeV})$ & $3.11 \times 10^{6}$ & $4.35 \times 10^{6}$ \\
\hline$p P b(\sqrt{s}=5 \mathrm{TeV})$ & 449.45 & 536.43 \\
$p P b(\sqrt{s}=8.8 \mathrm{TeV})$ & 535.32 & 678.46 \\
$p P b(\sqrt{s}=63 \mathrm{TeV})$ & 851.82 & 1408.95 \\
\hline$p p(\sqrt{s}=500 \mathrm{GeV})$ & 0.047 & 0.051 \\
$p p(\sqrt{s}=7 \mathrm{TeV})$ & 0.14 & 0.18 \\
$p p(\sqrt{s}=13 \mathrm{TeV})$ & 0.16 & 0.23 \\
$p p(\sqrt{s}=14 \mathrm{TeV})$ & 0.17 & 0.24 \\
$p p(\sqrt{s}=100 \mathrm{TeV})$ & 0.24 & 0.47 \\
\hline \hline
\end{tabular}

Tab. 6.1: Seções de choque totais para a produção dupla de $\rho$ em interações $\gamma \gamma$ em colisões $p p, p P b$ and $\mathrm{PbPb}$ nas energias do RHIC, LHC, FCC e CEPC-SPPC. Valores em $n b$.

acreditamos que os futuros colisores podem ser usados para estudar estes tipos de processos.

\subsection{Conclusões}

Nesse capítulo estudamos a produção dupla de mésons vetoriais devido à interação $\gamma \gamma$. 


\begin{tabular}{lll}
\hline \hline & Low energy & Low energy + IIM-S \\
\hline \hline$P b P b(\sqrt{s}=500 \mathrm{GeV})$ & 5640 & 6423 \\
$P b P b(\sqrt{s}=2.76 \mathrm{TeV})$ & 116550 & 235565 \\
$\mathrm{PbPb}(\sqrt{s}=5.5 \mathrm{TeV})$ & 217019 & 658589 \\
$\mathrm{PbPb}(\sqrt{s}=39 \mathrm{TeV})$ & 578195 & 6861251 \\
\hline$p P b(\sqrt{s}=5 \mathrm{TeV})$ & 64 & 310 \\
$p P b(\sqrt{s}=8.8 \mathrm{TeV})$ & 86 & 607 \\
$p P b(\sqrt{s}=63 \mathrm{TeV})$ & 172 & 4309 \\
\hline$p p(\sqrt{s}=500 \mathrm{GeV})$ & 0.0038 & 0.0085 \\
$p p(\sqrt{s}=7 \mathrm{TeV})$ & 0.023 & 0.24 \\
$p p(\sqrt{s}=13 \mathrm{TeV})$ & 0.029 & 0.45 \\
$p p(\sqrt{s}=14 \mathrm{TeV})$ & 0.030 & 0.48 \\
$p p(\sqrt{s}=100 \mathrm{TeV})$ & 0.050 & 2.42 \\
\hline \hline
\end{tabular}

Tab. 6.2: Seções de choque totais para a produção dupla de $J / \psi$ em interações $\gamma \gamma$ em colisões $p p, p P b$ and $\mathrm{PbPb}$ nas energias do RHIC, LHC, FCC e CEPC-SPPC. Valores em $p b$.

Para isso, utilizamos o formalismo de dipolo de cor, modelando $\sigma_{\gamma \gamma}$ em termos de $\sigma_{d d}$. Estudamos o comportamento das seções de choque com a energia e virtualidade para a produção de diversas combinações de mésons no estado final. Esse estudo pode ser bastante útil para aumentar nosso conhecimento sobre a transição entre processos hard e soft. Tais processos poderão ser estudados no ILC. Mostramos também como esse tipo de processo pode ser aplicado em colisões ultraperiféricas entre dois hádrons através da aproximação de fótons equivalentes. Isso se torna extremamente interessante, pois viabiliza o estudo da produção dupla de mésons vetoriais em interações $\gamma \gamma$ no LHC. Aqui estudamos a produção dupla de $\rho$ e $J / \psi$ considerando também efeitos de baixas energias discutidos recentemente na literatura. Nossos resultados indicam que a produção dupla de $\rho$ é dominada pelos efeitos de baixas energias, enquanto que os efeitos de altas energias são negligenciáveis. Por outro lado, os efeitos de altas energias para a produção de $J / \psi J / \psi$ são importantes já para energias do LHC e se tornam ainda mais visíveis em energias de futuros colisores hadrônicos. 


\section{Capítulo 7}

\section{Produção dupla de mésons vetoriais em interações $\gamma h$ no LHC}

Neste capítulo, estudamos a produção dupla de mésons vetoriais em processos fóton hádron $(\gamma h)$ em colisões $p p, p A$ e $A A$ e vamos apresentar nossas previsões para a produção de $\rho \rho, J / \psi J / \psi$ e $\rho J / \psi$ através do mecanismo de duplo espalhamento ("double scattering mechanism" - DSM). Vamos calcular as seções de choque totais e as distribuições de rapidez em energias do LHC. Em particular vamos apresentar, pela primeira vez, a produção dupla de mésons vetoriais em colisões $p p$ e $p A$ devido ao mecanismo de duplo espalhamento. Nossos resultados foram publicados na Ref. [5].

\subsection{O mecanismo de duplo espalhamento}

No capítulo 5, estudamos a produção de um único méson vetorial em colisões $\gamma h$. Já no capítulo 6, estudamos a produção dupla de mésons vetoriais em colisões $\gamma \gamma$. Contudo, em interações $\gamma h$ também pode ocorrer a produção dupla de mésons vetoriais. Este processo ocorre quando, em um mesmo evento, temos um duplo espalhamento $\gamma h$ e uma configuração possível é mostrada na Fig. 7.1 (devemos considerar que os fótons podem ser emitidos pelos hádrons 1 e 2 em todas as configurações possíveis). Além disso, como as seções de choque $\gamma p(A)$ para a produção de um único méson vetorial é grande para vários casos, os processos onde ocorrem múltiplas interações $\gamma p(A)$ podem ter uma probabilidade considerável.

Esses processos já foram estudados na literatura na Ref.[75] para várias combinações de mésons no estado final e na Ref.[118] onde um estudo detalhado foi feito para a produção dupla de $\rho$. Nas Refs. [75, 118], o modelo VDM foi utilizado para estimar as seções de choque $\gamma A$. Neste trabalho, estudamos a produção de $\rho \rho, \rho J / \psi$ e $J / \psi J / \psi$ em colisões periféricas $p p, p P b$ e $P b P b$ (coerentes) e usamos o formalismo de dipolo para o cálculo das seções de choque $\gamma p(A)$.

Portanto, para estimar a produção dupla de mésons vetoriais, vamos seguir as Refs. [75, 118], onde o DSM é escrito em termos das seções de choque de produção de um único méson vetorial (em um único espalhamento $\gamma h$, que será denotado por SSM - "single scattering mechanism"). Dessa forma, a produção de um méson vetorial $V_{1}$ com rapidez $y_{1}$ e de um 


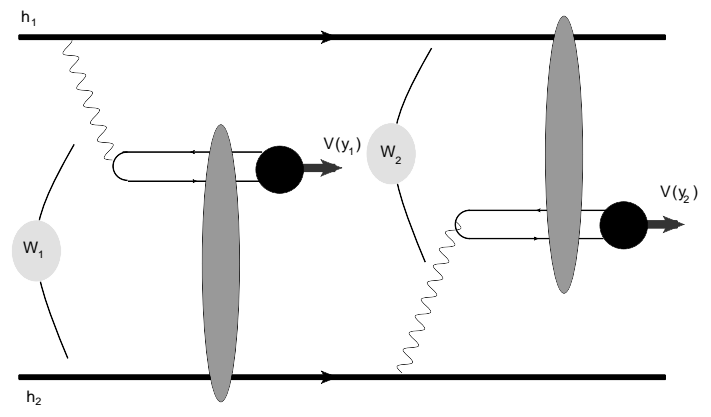

Fig. 7.1: Produção dupla de mésons vetoriais através do duplo espalhamento $\gamma h$.

segundo méson $V_{2}$ com rapidez $y_{2}$ devido ao processo DSM pode ser escrita como

$$
\frac{\mathrm{d}^{2} \sigma_{h_{1} h_{2} \rightarrow h_{1} V_{1} V_{2} h_{2}}}{\mathrm{~d} y_{1} \mathrm{~d} y_{2}}=\mathcal{C} \int_{b_{\text {min }}} \frac{\mathrm{d} \sigma_{h_{1} h_{2} \rightarrow h_{1} V_{1} h_{2}}}{\mathrm{~d}^{2} b \mathrm{~d} y_{1}} \times \frac{\mathrm{d} \sigma_{h_{1} h_{2} \rightarrow h_{1} V_{2} h_{2}}}{\mathrm{~d}^{2} b \mathrm{~d} y_{2}} \mathrm{~d}^{2} b
$$

onde $\mathcal{C}=1(1 / 2)$ para $V_{1} \neq V_{2}\left(V_{1}=V_{2}\right)$ e a seção de choque diferencial do processo SSM é definida por (analogamente ao que foi feito no cap. 5)

$$
\frac{\mathrm{d} \sigma_{h_{1} h_{2} \rightarrow h_{1} V h_{2}}}{\mathrm{~d}^{2} b \mathrm{~d} y}=\omega_{1} N\left(\omega_{1}, b\right) \sigma_{\gamma_{1} h_{2} \rightarrow V h_{2}}\left(\omega_{1}\right)+\omega_{2} N\left(\omega_{2}, b\right) \sigma_{\gamma_{2} h_{1} \rightarrow V h_{1}}\left(\omega_{2}\right)
$$

Nas equações acima $b$ é o parâmetro de impacto da colisão $h_{1} h_{2}, b_{\min }>R_{1}+R_{2}$ e

$$
\omega_{1}=\frac{M_{V}}{2} e^{y} \text { e } \omega_{2}=\frac{M_{V}}{2} e^{-y} .
$$

O fluxo de fótons equivalentes dependente de $b$ e $\omega$ é dado pela Eq. (6.10). Usamos o fator de forma igual a 1 para o caso em que a fonte de fótons é o $\mathrm{Pb}$ e o fator de forma de tipo dipolo para o caso onde o próton atua como fonte de fótons (maiores detalhes podem ser encontrados no apêndice C).

Para calcular a seção de choque para o processo SSM via Eq. (7.2) (e, consequentemente para o processo DSM via Eq. (7.1)), é necessário conhecer a seção de choque $\sigma_{\gamma h \rightarrow V h}(\omega)$, que pode ser calculda pelo formalismo desenvolvido no capítulo 5. Aqui, para a amplitude de espalhamento dipolo-hádron $\mathcal{N}$ utilizamos o modelo bCGC NEW.

\subsection{Previsões para o LHC}

Nesta seção vamos apresentar nossas previsões para o DSM. Na Fig. 7.2 mostramos nossos resultados para as seções de choque de produção dupla de mésons vetoriais devido 

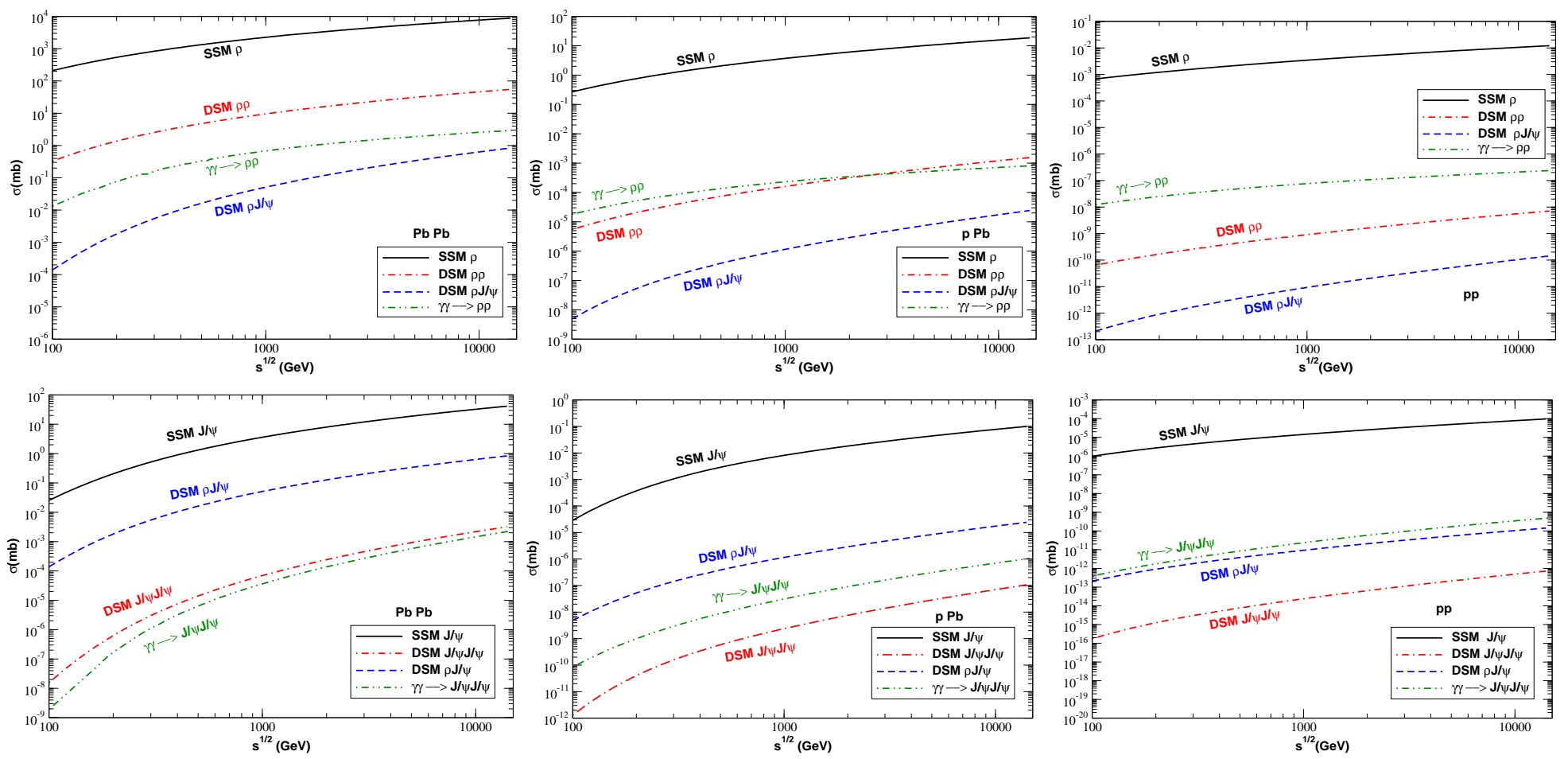

Fig. 7.2: Dependência da seção de choque com a energia para a produção dupla de mésons vetoriais através dos mecanismos de duplo espalhamento e de interação $\gamma \gamma$.

\begin{tabular}{||c|c|c|c|c||}
\hline \hline Colisão & Mecanismo & $\rho \rho$ & $J / \psi J / \psi$ & $\rho J / \psi$ \\
\hline \hline$P b P b(\sqrt{s}=2.76 \mathrm{TeV})$ & $\mathrm{DSM}$ & $21.150 \mathrm{mb}$ & $402.301 \mathrm{nb}$ & $0.18 \mathrm{mb}$ \\
& $\gamma \gamma$ & $1.389 \mathrm{mb}$ & $235.565 \mathrm{nb}$ & - \\
\hline $\mathrm{PbPb}(\sqrt{s}=5.5 \mathrm{TeV})$ & $\mathrm{DSM}$ & $29.421 \mathrm{mb}$ & $1054.951 \mathrm{nb}$ & $0.35 \mathrm{mb}$ \\
& $\gamma \gamma$ & $1.973 \mathrm{mb}$ & $658.589 \mathrm{nb}$ & - \\
\hline \hline$p P b(\sqrt{s}=5 \mathrm{TeV})$ & $\mathrm{DSM}$ & $702.595 \mathrm{nb}$ & $28.473 \mathrm{pb}$ & $8.929 \mathrm{nb}$ \\
& $\gamma \gamma$ & $537.432 \mathrm{nb}$ & $310.194 \mathrm{pb}$ & - \\
\hline \hline$p p(\sqrt{s}=7 \mathrm{TeV})$ & $\mathrm{DSM}$ & $4.354 \mathrm{pb}$ & $3.223 \times 10^{-4} \mathrm{pb}$ & $7.469 \times 10^{-2} \mathrm{pb}$ \\
& $\gamma \gamma$ & $182.442 \mathrm{pb}$ & $0.2412 \mathrm{pb}$ & - \\
\hline$p p(\sqrt{s}=14 \mathrm{TeV})$ & $\mathrm{DSM}$ & $7.083 \mathrm{pb}$ & $7.256 \times 10^{-4} \mathrm{pb}$ & $14.288 \times 10^{-2} \mathrm{pb}$ \\
& $\gamma \gamma$ & $237.006 \mathrm{pb}$ & $0.4793 \mathrm{pb}$ & - \\
\hline \hline
\end{tabular}

Tab. 7.1: Seções de choque totais para a produção dupla de mésons vetoriais através dos mecanismos de duplo espalhamento e de interação $\gamma \gamma$ em energias do LHC.

aos processos DSM (duplo espalhamento $\gamma h$ ) e $\gamma \gamma$ (o qual foi discutido no capítulo anterior) e comparamos também com a produção de um único méson criado no processo SSM. Para a produção dupla de $\rho$ (gráficos superiores), o DSM domina frente ao $\gamma \gamma$ em colisões $P b P b$. Por outro lado, esses mecanismos são comparáveis em colisões $p P b$ e, em colisões $p p$ o processo $\gamma \gamma$ passa a dominar. Para a produção dupla de $J / \psi$ (gráficos inferiores), os mecanismos 

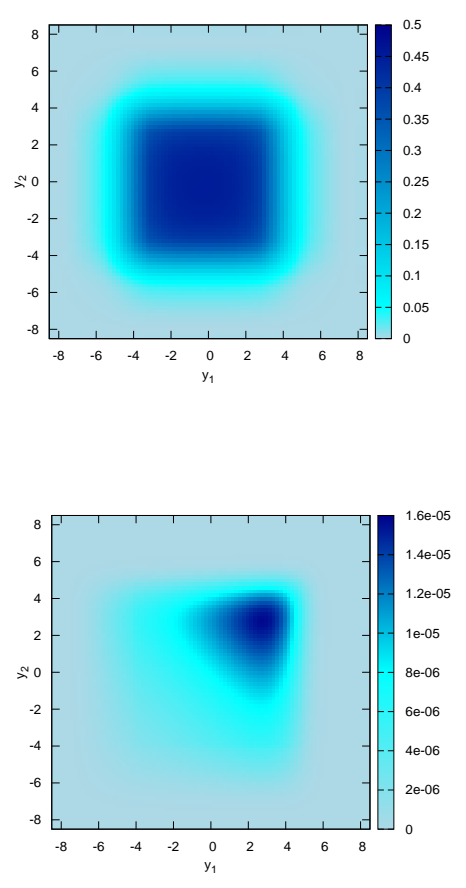
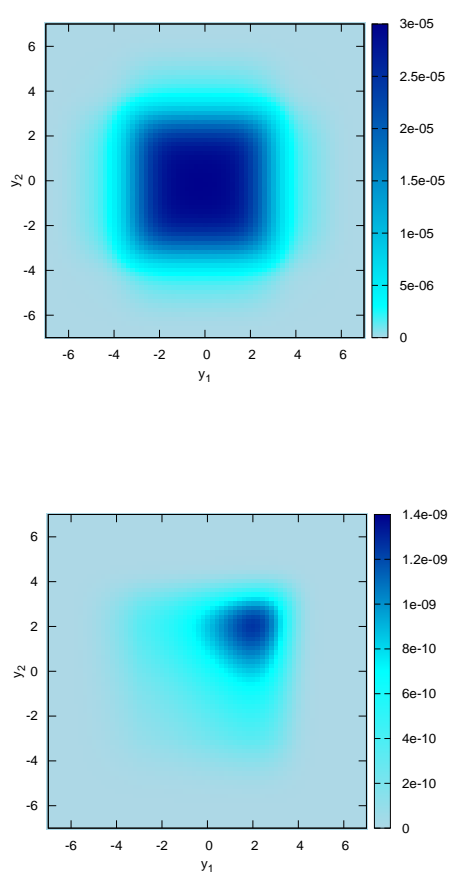
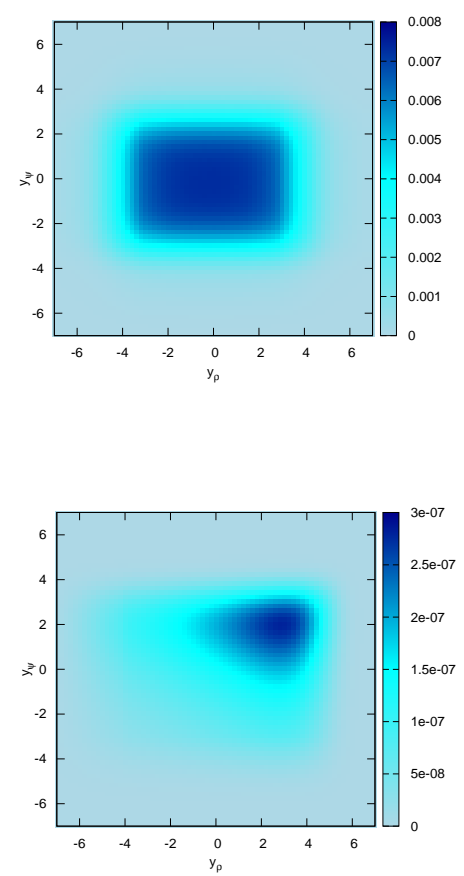

Fig. 7.3: Distribuições de rapidez para a produção dupla de $\rho$ (primeira coluna), $J / \psi$ (segunda coluna) e para a produção mista $\rho J / \psi$ (terceira coluna) devido ao mecanismo de duplo espalhamento em colisões $\mathrm{PbPb} a \sqrt{s}=5.5 \mathrm{TeV}$ (painel superior) e em colisões $\mathrm{pPb} a \sqrt{s}=5 \mathrm{TeV}$ (painel inferior). As distribuições de rapidez estão em $m b$.

DSM e $\gamma \gamma$ fornecem predições bastante semelhantes no caso $P b P b$, embora as predições feitas com o DSM sejam maiores. Para colisões $p P b(p p)$, o processo DSM fornece seções de choque que são um fator da ordem de 10(100) menores do que as obtidas com o processo $\gamma \gamma$. Além disso, podemos observar que a produção mista de $\rho$ e $J / \psi$ devido ao processo DSM é importante em energias do LHC. Na Tab. 7.1, mostramos nossas previsões para as seções de choque de produção dupla de mésons vetoriais devido aos dois mecanismos em colisões $p p, p P b$ e $P b P b$ para energias do LHC.

Na Fig. 7.3 apresentamos nossas previsões para as distribuições de rapidez para a produção dupla de mésons vetoriais em colisões $P b P b$ e $p P b$ via DSM. Para as colisões $\mathrm{PbPb}$ (painel superior) temos uma distribuição simétrica no caso da produção dupla de $\rho$ e $J / \psi$ (primeira e segunda coluna), enquanto que para a produção mista $\rho J / \psi$ a distribuição é assimétrica, sendo maior para a rapidez associada ao $\rho$. No caso de colisões $p P b$ (painel inferior), observamos uma assimetria associada às diferentes fontes de fótons para todos os casos. Para o caso da produção mista $\rho J / \psi$ (terceira coluna) temos também a assimetria devido aos diferentes estados finais produzidos e, como no caso $\mathrm{PbPb}$, a distribuição de rapidez associada ao $\rho$ é maior do que a do $J / \psi$. 


\subsection{Conclusões}

O fato de que seções de choque para a produção de um único méson vetorial possam ser muito grandes, se reflete na ocorrência de seções de choque para a produção dupla de mésons devido ao duplo espalhamento $\gamma h$ (DSM) com valores não desprezíveis. Nesse capítulo discutimos o DSM para a produção de $\rho \rho, \rho J / \psi$ e $J / \psi J / \psi$ em colisões ultraperiféricas para energias do LHC. Comparamos esses resultados com os do capítulo anterior devido ao processo $\gamma \gamma$. Nossos resultados indicam que o processo DSM domina o $\gamma \gamma$ em colisões $\mathrm{PbPb}$, é comparável em colisões $p P b$ e é menor em colisões $p p$. Portanto as colisões com diferentes projéteis podem ser úteis para separar a produção dupla devido a cada um desses processos. 


\section{Capítulo 8}

\section{Conclusão}

Neste trabalho estudamos a produção exclusiva de mésons vetoriais em colisões ultraperiféricas hádron-hádron através dos subprocessos $\gamma h$ e $\gamma \gamma$. Nos processos $\gamma h$, estudamos a produção de um (single scattering mechanism) e de dois mésons vetoriais, devido a dois espalhamentos $\gamma h$ na mesma colisão (double scattering mechanism). Além disso, a produção dupla de mésons vetoriais foi também estudada no processo $\gamma \gamma \rightarrow V V$ (onde $V$ denota o méson vetorial) e estendida para colisores hadrônicos em colisões periféricas $A A \rightarrow A V V A$. Esses cálculos foram feitos com auxílio do formalismo de dipolo de cor e da aproximação de fótons equivalentes. Em particular, no formalismo de dipolo, utilizamos modelos da física de saturação para as seções de choque de interação dipolo-alvo. Fizemos, também, uma revisão referente aos métodos necessários para esses cáculos apresentando as principais características da QCD em altas energias e da produção de partículas em colisões ultraperiféricas. Nossos resultados para as seções de choque (totais e diferenciais) indicam que o estudo experimental dos processos discutidos é viável e pode servir para aumentar o nosso conhecimento a respeito da dinâmica da QCD em altas energias.

No capítulo 5 apresentamos nossas predições para a produção de um único méson vetorial em colisões ultraperiféricas. Mostramos que os modelos da física de saturação possuem boa concordância com os dados experimentais do LHC disponíveis até o momento. Esse fato é extremamente significativo, tendo em vista que: (i) os modelos da física de saturação têm sucesso em descrever, também, outros processos e características da QCD em altas energias (DIS inclusivo e difrativo, scaling geométrico, dependência da multiplicidade hadrônica com a energia, entre vários outros) e (ii) que os modelos fenomenológicos utilizados foram fixados por dados de HERA e, portanto, não tiveram nenhum parâmetro alterado para descrever os dados recentes do LHC. Em especial, mostramos que na região de rapidez central as diferenças entre modelos são ampliadas com o aumento de $\sqrt{s}$.

No capítulo 6, calculamos as seções de choque para a produção dupla de mésons vetoriais em colisões fóton-fóton. Para tanto, fixamos um único modelo de função de onda do méson vetorial (Gaus-LC) enquanto usamos dois modelos para a seção de choque dipolo-próton e dois modelos para a seção de choque dipolo-dipolo. Estimamos o comportamento das seções de choque com a energia e com a virtualidade. Observamos que as maiores diferenças entre as predições se dão pela escolha do modelo da seção de choque dipolo-dipolo. Argumentamos que esse processo poderá ser estudado no futuro colisor $e^{+} e^{-}$ILC. Logo após, mostramos como esse processo pode ser estudado no LHC através de colisões periféricas $p p, p P b$ e $\mathrm{PbPb}$, aplicando o método de fótons equivalentes em produção dupla de $\rho$ e $J / \psi$. Para 
isso, utilizamos nossos resultados para as seções de choque $\gamma \gamma$ calculadas com o formalismo de dipolo, para altas energias, combinadas com seções de choque de processos de baixas energias presentes na literatura. Nossos resultados sugerem que a produção dupla de $J / \psi$ sofre maiores efeitos da física de altas energias comparada com a produção dupla de $\rho$, a qual é dominada por processos de baixas energias.

No capítulo 7, estudamos a produção dupla de mésons vetoriais devido ao mecanismo de duplo espalhamento (denotado por DSM) em colisões ultraperiféricas. Conforme discutido no texto, esse processo ocorre devido ao duplo espalhamento $\gamma h$ em uma mesma colisão hh e, desta forma, é baseado no formalismo utilizado no cap.5. Apresentamos nossas predições para a produção de $\rho \rho, J / \psi J / \psi$ e $\rho J / \psi$. Para os casos de produção dupla de $\rho$ e $J / \psi$, comparamos nossos resultados com os do processo $\gamma \gamma$. Mostramos que os processos DSM dominam os processos $\gamma \gamma$ em colisões $P b P b$, são comparáveis em colisões $p P b$ e são dominados pelos processos $\gamma \gamma$ em colisões $p p$. Argumentamos que o estudo desses processos com diferentes projéteis podem ser interessantes no auxílio da separação experimental dos mesmos.

Em resumo, mapeamos alguns dos principais processos de fotoprodução de mésons vetoriais que podem ser estudados no LHC e em futuros colisores nas chamadas colisões ultraperiféricas. As interações $\gamma h$ e $\gamma \gamma$ foram estudadas via formalismo de dipolo de cor com modelos da física de saturação (ou física do CGC). Os dados experimentais atuais para colisões ultraperiféricas hádron-hádron mostram que tal estudo é possível e pode servir para vincular a dinâmica da QCD em altas energias. É interessante notar, como já mencionado anteriormente, que a física do CGC é universal e aplicável, com sucesso, em inúmeros experimentos. Portanto consideramos extremamente importante esta exploração de colisões periféricas que vem sendo feita tanto do ponto de vista fenomenológico quanto do ponto de vista experimental, a fim de buscar estender o conjunto de processos estudados pela física do CGC.

Por fim, existem várias extensões possíveis aos nossos trabalhos. Podemos estender nossos resultados para energias de futuros colisores e considerar outros modelos de $\mathcal{N}$ e $\psi_{V}$ além de diferentes modelos para efeitos e correções para a produção de um único méson vetorial. Em nossos trabalhos para a produção dupla de mésons vetoriais, podemos utilizar fatores de forma mais realistas para o cálculo do fluxo de fótons equivalentes. Além disso, futuramente, pretendemos estudar a fotoprodução de dijatos no LHC e em futuros colisores e ampliar nossa compreensão dos efeitos de múltiplos espalhamentos em interações coerentes e incoerentes. 
Apêndices 


\section{Apêndice A}

\section{Funções de onda do cone de luz}

\section{A.1 Função de onda do fóton}

Nesse apêndice, vamos seguir as regras para a construção de funções de onda do cone de luz para o fóton enunciadas em [119] e os cálculos presentes em [120]. A Fig.A.1 mostra a diagrama correspondente à função de onda do cone de luz de um fóton flutuando em um par quark-antiquark.

Para o cálculo da função de onda um fóton com momentum $q=\left(q^{+}, q^{-}=-\frac{Q^{2}}{2 q^{+}}, \vec{q}_{T}=0\right)$, na notação do cone de luz (como definido na Ref. [18]), multiplica-se:

- um fator de cor $\sqrt{N_{C}}$

- um fator de sabor $e_{f}$

- um termo de espinor

$$
\bar{u}_{h}\left(z q^{+}, \vec{k}\right) \varepsilon_{\mu}(q, \lambda) \gamma^{\mu} v_{\bar{h}}\left((1-z) q^{+},-\vec{k}\right)
$$

- um fator $\left(\sqrt{2} z q^{+}\right)^{-1 / 2}\left(\sqrt{2}(1-z) q^{+}\right)^{-1 / 2}$ para linhas de quark e antiquark

- um denominador de energia

$$
-\sqrt{2} q^{+}\left[Q^{2}+\frac{\vec{k}^{2}+m^{2}}{z(1-z)}\right]^{-1}
$$

Além disso, os vetores polarização são definidos como

$$
\begin{aligned}
\varepsilon_{L} & =\left(\frac{q^{+}}{Q}, \frac{Q}{2 q^{+}}, \overrightarrow{0}\right) \\
\varepsilon_{T}^{\lambda}=-\frac{1}{\sqrt{2}}\left(0,0, \vec{\varepsilon}_{T}^{\lambda}\right) & \rightarrow \vec{\varepsilon}_{T}^{\lambda}=-\frac{1}{\sqrt{2}}(1, i \lambda)
\end{aligned}
$$

na notação $(+,-, \perp)$ e $\lambda= \pm 1$.

Os elementos de matriz espinorial do cone de luz são dados por

$$
\bar{u}_{h} \gamma^{+} v_{\bar{h}}=2 \sqrt{z(1-z)} q^{+} \delta_{h,-\bar{h}},
$$




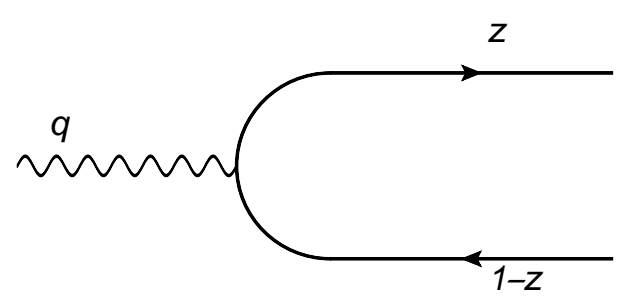

Fig. A.1: Diagrama para a função de onda do cone de luz do fóton.

$$
\begin{gathered}
\bar{u}_{h} \gamma^{-} v_{\bar{h}}=-\frac{\vec{k}^{2}+m^{2}}{\sqrt{z(1-z)} q^{+}} \delta_{h,-\bar{h}}, \\
\bar{u}_{h} \gamma^{i} v_{\bar{h}}=\frac{(1-2 z) k^{i} \mp i \varepsilon^{i j 3} k^{j}}{\sqrt{z(1-z)}} \delta_{h,-\bar{h}} \mp m \frac{\delta^{i 1} \mp i \delta^{i 2}}{\sqrt{z(1-z)}} \delta_{h, \bar{h}},
\end{gathered}
$$

onde nos termos com $\mp$, usamos o sinal superior para $h=1 / 2$ e o sinal inferior para $h=-1 / 2$.

Dessa forma, a função de onda para o fóton será dada por

$$
\begin{aligned}
\psi_{h, \bar{h}}^{\lambda}(z, \vec{k}) & =-\sqrt{N_{C}} e e_{f}\left(\frac{1}{2 z q^{+}(1-z) q^{+}}\right)^{1 / 2} \frac{\left(-\sqrt{2} q^{+}\right)}{Q^{2} z(1-z)+\vec{k}^{2}+m^{2}} z(1-z) \bar{u} \varepsilon \cdot \gamma v \\
& =-\sqrt{N_{C}} e e_{f} \frac{z(1-z)}{\sqrt{z(1-z)}} \frac{\bar{u} \varepsilon \cdot \gamma v}{Q^{2} z(1-z)+\vec{k}^{2}+m^{2}} \\
& =-\sqrt{N_{C}} e e_{f} \sqrt{z(1-z)} \varepsilon_{\mu}^{\lambda} \frac{\bar{u} \gamma^{\mu} v}{\vec{k}^{2}+\epsilon^{2}}
\end{aligned}
$$

onde na última linha definimos $\epsilon^{2}=Q^{2} z(1-z)+m^{2}$.

Vamos a partir de agora estudar as polarizações longitudinal e transversa separadamente.

\section{A.1.1 Polarização longitudinal}

O produto escalar $\varepsilon_{\mu}^{L} \gamma^{\mu}$ é dado por

$$
\varepsilon_{\mu}^{L} \gamma^{\mu}=\varepsilon^{L+} \gamma^{-}+\varepsilon^{L-} \gamma^{+}
$$


e, usando as Eqs.(A.4) e (A.5), obtemos

$$
\bar{u} \varepsilon^{-} \gamma^{+} v=\left(\frac{Q}{2 q^{+}}\right) 2 \sqrt{z(1-z)} q^{+} \delta_{h,-\bar{h}}=\sqrt{z(1-z)} Q \delta_{h,-\bar{h}}
$$

$\mathrm{e}$

$$
\bar{u} \varepsilon^{+} \gamma^{-} v=\left(\frac{q^{+}}{Q}\right)\left(-\frac{\vec{k}^{2}+m^{2}}{\sqrt{z(1-z)} q^{+}}\right) \delta_{h,-\bar{h}}=-\frac{\vec{k}^{2}+m^{2}}{Q \sqrt{z(1-z)}} \delta_{h,-\bar{h}}
$$

Dessa forma

$$
\begin{aligned}
\psi^{L} & =-\frac{\sqrt{N_{C}} e e_{f} \sqrt{z(1-z)}}{\vec{k}^{2}+\epsilon}\left[\sqrt{z(1-z)} Q+(\sqrt{z(1-z)} Q-\sqrt{z(1-z)} Q)-\frac{\vec{k}^{2}+m^{2}}{\sqrt{z(1-z)} Q}\right] \delta_{h,-\bar{h}} \\
& =-\frac{\sqrt{N_{C}} e e_{f} \sqrt{z(1-z)}}{\vec{k}^{2}+\epsilon}\left[2 \sqrt{z(1-z)} Q-\frac{\vec{k}^{2}+m^{2}+z(1-z) Q^{2}}{\sqrt{z(1-z)} Q}\right] \delta_{h,-\bar{h}} \\
& =-\sqrt{N_{C}} e e_{f}\left[\frac{2 z(1-z) Q}{\vec{k}^{2}+\epsilon}-\frac{1}{Q}\right] \delta_{h,-\bar{h}} .
\end{aligned}
$$

Vamos agora fazer uma transformada de Fourier da equação acima a fim de obter a função de onda no espaço das posições. O segundo termo da equação acima produzirá uma $\delta^{2}(\vec{r})$ ao efetuarmos a transformada de Fourier, que forçará um dipolo com separação nula, o qual não interage com um alvo via interação forte (limite de transparência de cor). Portanto esse termo deverá ser desconsiderado de nossas contas. Além disso, a transformada de Fourier atua na função

$$
f(\vec{k})=\frac{1}{\vec{k}^{2}+\epsilon^{2}},
$$

Assim, a transformada de Fourier fica

$$
\begin{aligned}
\int e^{i \vec{k} \cdot \vec{r}} \frac{1}{\vec{k}^{2}+\epsilon^{2}} \frac{d^{2} \vec{k}}{(2 \pi)^{2}} & =\int_{0}^{\infty}\left[\int_{0}^{2 \pi} e^{i|\vec{k}||\vec{r}| \cos \varphi} d \varphi\right] \frac{|\vec{k}| d|\vec{k}|}{(2 \pi)^{2}} \frac{1}{|\vec{k}|^{2}+\epsilon^{2}} \\
& =\int_{0}^{\infty}\left[2 \pi J_{0}(|\vec{k}||\vec{r}|)\right] \frac{|\vec{k}| d|\vec{k}|}{(2 \pi)^{2}} \frac{1}{|\vec{k}|^{2}+\epsilon^{2}} \\
& =\frac{1}{2 \pi} \int_{0}^{\infty}|\vec{k}| J_{0}(|\vec{k}||\vec{r}|) \frac{d|\vec{k}|}{|\vec{k}|^{2}+\epsilon^{2}} \\
& =\frac{K_{0}(\epsilon r)}{2 \pi},
\end{aligned}
$$

onde usamos

$$
\int_{0}^{2 \pi} e^{i|\vec{k}| \vec{r} \mid \cos \varphi} d \varphi=2 \pi J_{0}(|\vec{k}||\vec{r}|)
$$


$\mathrm{e}$

$$
\int_{0}^{\infty} d t \frac{t^{\nu+1} J_{\nu}(a t)}{\left(t^{2}+z^{2}\right)^{\mu+1}}=\frac{a^{\mu} z^{\nu-\mu}}{2^{\mu} \Gamma(\mu+1)} K_{\nu-\mu}(a z) .
$$

Dessa forma, a função de onda para a polarização longitudinal dos fótons no espaço das posições é dada por (dividindo pelo fator de normalização $\sqrt{4 \pi}$ )

$$
\psi^{L}(z, r)=-\sqrt{\frac{N_{C}}{4 \pi}} e e_{f} 2 z(1-z) Q \frac{K_{0}(\epsilon r)}{2 \pi} \delta_{h,-\bar{h}} .
$$

Tomando o módulo quadrado e somando sobre os spins do par $q \bar{q}$, obtemos $\left(e^{2}=4 \pi \alpha_{e m}\right)$

$$
\left|\psi^{L}(z, r)\right|^{2}=\sum_{f} \frac{N_{C}}{4 \pi} \frac{4 \pi}{\pi^{2}} \alpha_{e m} e_{f}^{2} z^{2}(1-z)^{2} Q^{2} K_{0}^{2}(\epsilon r) \sum_{h} \sum_{\bar{h}} \delta_{h,-\bar{h}} \delta_{h,-\bar{h}}
$$

e, como

$$
\sum_{h} \sum_{\bar{h}} \delta_{h,-\bar{h}} \delta_{h,-\bar{h}}=\sum_{\bar{h}} \delta_{-\bar{h},-\bar{h}}=\delta_{-1 / 2,-1 / 2}+\delta_{1 / 2,1 / 2}=2
$$

obtemos

$$
\left|\psi^{L}(z, r)\right|^{2}=\frac{2 N_{C}}{\pi^{2}} \sum_{f} \alpha_{e m} e_{f}^{2} z^{2}(1-z)^{2} Q^{2} K_{0}^{2}(\epsilon r) .
$$

\section{A.1.2 Polarização transversal}

O produto escalar $\varepsilon_{\mu}^{T} \gamma^{\mu}$ é dado por

$$
\varepsilon_{\mu}^{T} \gamma^{\mu}=-\vec{\varepsilon}_{T}^{\lambda} \cdot \vec{\gamma}=-\varepsilon_{T}^{(\lambda) i} \gamma^{i}
$$

Além disso, da forma como foi definido o vetor polarização e o elemento de matriz $\bar{u} \gamma^{i} v$, teremos que efetuar os seguintes produtos:

$$
\vec{\varepsilon}_{T}^{\lambda} \cdot \vec{k}=-\frac{1}{\sqrt{2}}\left[k^{1}+i \lambda k^{2}\right]
$$

e

$$
\begin{aligned}
\varepsilon^{1} k^{2}-\varepsilon^{2} k^{1} & =-\frac{1}{\sqrt{2}}\left[k^{2}-i \lambda k^{1}\right] \\
& =-i \lambda\left\{-\frac{1}{\sqrt{2}}\left[k^{1}+i \lambda k^{2}\right]\right\} \\
& =-i \lambda \vec{\varepsilon}_{T}^{\lambda} \cdot \vec{k}
\end{aligned}
$$

onde usamos o fato de que $\lambda X=X / \lambda$, pois $\lambda= \pm 1$.

Vamos usar a Eq.(A.6) e analisar separadamente os casos $h=\bar{h}$ e $h=-\bar{h}$. 
Caso $\mathbf{h}=-\overline{\mathbf{h}}$

Nesse caso temos

$$
\begin{aligned}
-\varepsilon_{T}^{(\lambda) i} \bar{u} \gamma^{i} v & =-\left\{\frac{(1-2 z) \vec{\varepsilon}_{T}^{\lambda} \cdot \vec{k} \mp i\left(\varepsilon^{1} k^{2}-\varepsilon^{2} k^{1}\right)}{\sqrt{z(1-z)}}\right\} \\
& =-\left\{\frac{(1-2 z) \mp \lambda}{\sqrt{z(1-z)}}\right\}\left(\vec{\varepsilon}_{T}^{\lambda} \cdot \vec{k}\right) \\
& =\frac{1}{\sqrt{2}} \frac{(1-2 z) \mp \lambda}{\sqrt{z(1-z)}}\left[k^{1}+i \lambda k^{2}\right] .
\end{aligned}
$$

Analisando para cada polarização de fóton e par $q \bar{q}$ possvel separadamente, obtemos

- $\lambda=+, h=+, \bar{h}=-$

$$
-\varepsilon_{T}^{(\lambda) i} \bar{u} \gamma^{i} v=-\frac{\sqrt{2} z}{\sqrt{z(1-z)}}\left[k^{1}+i k^{2}\right]
$$

- $\lambda=+, h=-, \bar{h}=+$

$$
-\varepsilon_{T}^{(\lambda) i} \bar{u} \gamma^{i} v=\frac{\sqrt{2}(1-z)}{\sqrt{z(1-z)}}\left[k^{1}+i k^{2}\right]
$$

- $\lambda=-, h=+, \bar{h}=-$

$$
-\varepsilon_{T}^{(\lambda) i} \bar{u} \gamma^{i} v=\frac{\sqrt{2}(1-z)}{\sqrt{z(1-z)}}\left[k^{1}-i k^{2}\right]
$$

- $\lambda=-, h=-, \bar{h}=+$

$$
-\varepsilon_{T}^{(\lambda) i} \bar{u} \gamma^{i} v=-\frac{\sqrt{2} z}{\sqrt{z(1-z)}}\left[k^{1}-i k^{2}\right] .
$$

Caso $\mathbf{h}=\overline{\mathbf{h}}$

Nesse caso temos

$$
\begin{aligned}
-\varepsilon_{T}^{(\lambda) i} \bar{u} \gamma^{i} v & =\mp(-1) \frac{\left[\delta^{i 1} \mp i \delta^{i 2}\right]}{\sqrt{z(1-z)}} \varepsilon_{T}^{(\lambda) i} \\
& =\mp(-1) \frac{m[(-1 / \sqrt{2}) \mp(1 / \sqrt{2}) \lambda]}{\sqrt{z(1-z)}} \\
& =\mp \frac{m}{\sqrt{2}} \frac{[1 \pm \lambda]}{\sqrt{z(1-z)}} .
\end{aligned}
$$

Analisando para cada polarização de fóton e par $q \bar{q}$ possv́el separadamente, obtemos 
- $\lambda=+, h=+, \bar{h}=+$

$$
-\varepsilon_{T}^{(\lambda) i} \bar{u} \gamma^{i} v=-\frac{\sqrt{2} m}{\sqrt{z(1-z)}}
$$

- $\lambda=-, h=-, \bar{h}=-$

$$
-\varepsilon_{T}^{(\lambda) i} \bar{u} \gamma^{i} v=\frac{\sqrt{2} m}{\sqrt{z(1-z)}}
$$

- $(\lambda=+, h=\bar{h}=-)$ e $(\lambda=-, h=\bar{h}=+)$

$$
-\varepsilon_{T}^{(\lambda) i} \bar{u} \gamma^{i} v=0
$$

Juntando todos esses resultados em uma notação compacta, temos para $\psi_{h \bar{h}}^{T(\lambda=+)}(\vec{k}, z)$ e $\psi_{h \bar{h}}^{T(\lambda=-)}(\vec{k}, z)$

$$
\begin{aligned}
\psi_{h \bar{h}}^{T(\lambda=+)}(\vec{k}, z) & =\sqrt{2} \sqrt{N_{C}} e e_{f}\left\{\left[k_{1}+i k^{2}\right]\left(z \delta_{h+} \delta_{\bar{h}-}-(1-z) \delta_{h-} \delta_{\bar{h}+}\right)+m \delta_{h+} \delta_{\bar{h}+}\right\} \\
& \times \frac{1}{\vec{k}^{2}+\epsilon^{2}}
\end{aligned}
$$

e

$$
\begin{aligned}
\psi_{h \bar{h}}^{T(\lambda=-)}(\vec{k}, z) & =-\sqrt{2} \sqrt{N_{C}} e e_{f}\left\{\left[k_{1}-i k^{2}\right]\left((1-z) \delta_{h+} \delta_{\bar{h}-}-z \delta_{h-} \delta_{\bar{h}+}\right)+m \delta_{h+} \delta_{\bar{h}+}\right\} \\
& \times \frac{1}{\vec{k}^{2}+\epsilon^{2}}
\end{aligned}
$$

A transformada de Fourier atuará na função $f(\vec{k})$ [ver a Eq.(A.12)] que já foi calculada para o caso longitudinal e em $\left[k^{1} \pm i k^{2}\right] f(\vec{k})$, que produzirá

$$
\int \frac{d^{2} \vec{k}}{(2 \pi)^{2}} e^{i \vec{k} \cdot \vec{r}} \frac{k^{1} \pm i k^{2}}{\vec{k}^{2}+\epsilon^{2}}=\int \frac{d^{2} \vec{k}}{(2 \pi)^{2}} e^{i \vec{k} \cdot \vec{r}} \frac{k^{1}}{\vec{k}^{2}+\epsilon^{2}} \pm i \int \frac{d^{2} \vec{k}}{(2 \pi)^{2}} e^{i \vec{k} \cdot \vec{r}} \frac{k^{2}}{\vec{k}^{2}+\epsilon^{2}}
$$

mas

$$
\begin{aligned}
e^{i \vec{k} \cdot \vec{r}} & =e^{i k^{1} x+i k^{2} y} \\
\rightarrow k^{1} e^{i \vec{k} \cdot \vec{r}} & =-i \partial_{x} e^{i \vec{k} \cdot \vec{r}} \\
\rightarrow k^{2} e^{i \vec{k} \cdot \vec{r}} & =-i \partial_{y} e^{i \vec{k} \cdot \vec{r}} .
\end{aligned}
$$

Logo

$$
\int \frac{d^{2} \vec{k}}{(2 \pi)^{2}} e^{i \vec{k} \cdot \vec{r}} \frac{k^{1} \pm i k^{2}}{\vec{k}^{2}+\epsilon^{2}}=-i\left(\partial_{x} \pm i \partial_{y}\right) f(r),\left[f(r)=\frac{K_{0}(\epsilon r)}{2 \pi}\right]
$$


Na equação acima, $f(r)$ não possui dependência angular. Tratando $r$ como uma variável complexa, temos

$$
\begin{gathered}
r=x+i y=|r| e^{i \theta}=|r| \cos \theta+i|r| \operatorname{sen} \theta \\
r^{*} r=|r|^{2} \\
\rightarrow x=|r| \cos \theta ; y=|r| \operatorname{sen} \theta \\
\rightarrow \tan \theta=\frac{y}{x} \cdot|r|^{2}=x^{2}+y^{2}
\end{gathered}
$$

de forma que

$$
\begin{aligned}
& \rightarrow \partial_{x}=\cos \theta \partial_{r}-\frac{\operatorname{sen} \theta}{r} \partial_{\theta} \\
& \rightarrow \partial_{y}=\operatorname{sen} \theta \partial_{r}+\frac{\cos \theta}{r} \partial_{\theta} .
\end{aligned}
$$

Então a Eq.(A.35) fica

$$
\int \frac{d^{2} \vec{k}}{(2 \pi)^{2}} e^{i \vec{k} \cdot \vec{r}} \frac{k^{1} \pm i k^{2}}{\vec{k}^{2}+\epsilon^{2}}=-i(\cos \theta \pm i \operatorname{sen} \theta) \partial_{r} f(r)=-i e^{ \pm i \theta} \partial_{r} f(r)
$$

e usando o fato de que $K_{0}^{\prime}(X)=-K_{1}(X)$, obtemos

$$
\int \frac{d^{2} \vec{k}}{(2 \pi)^{2}} e^{i \vec{k} \cdot \vec{r}} \frac{k^{1} \pm i k^{2}}{\vec{k}^{2}+\epsilon^{2}}=i e^{ \pm i \theta} \epsilon \frac{K_{1}(\epsilon r)}{2 \pi}
$$
$\sqrt{4 \pi})$

As funções de onda $\psi^{T(\lambda= \pm)}(z, r)$ são dadas por (dividindo pelo fator de normalização

$$
\begin{array}{r}
\psi_{h \bar{h}}^{T(\lambda=+)}(\vec{r}, z)=\sqrt{2} \sqrt{\frac{N_{C}}{4 \pi}} e e_{f}\left\{i e^{i \theta} \epsilon\left(z \delta_{h+} \delta_{\bar{h}-}-(1-z) \delta_{h-} \delta_{\bar{h}+}\right) \frac{K_{1}(\epsilon r)}{2 \pi}\right. \\
\left.+m \delta_{h+} \delta_{\bar{h}+} \frac{K_{0}(\epsilon r)}{2 \pi}\right\}
\end{array}
$$

e

$$
\begin{aligned}
& \psi_{h \bar{h}}^{T(\lambda=-)}(\vec{r}, z)=-\sqrt{2} \sqrt{\frac{N_{C}}{4 \pi}} e e_{f}\left\{i e^{-i \theta} \epsilon\left((1-z) \delta_{h+} \delta_{\bar{h}-}-z \delta_{h-} \delta_{\bar{h}+}\right) \frac{K_{1}(\epsilon r)}{2 \pi}\right. \\
& \left.+m \delta_{h+} \delta_{\bar{h}+} \frac{K_{0}(\epsilon r)}{2 \pi}\right\}
\end{aligned}
$$


Tomando o módulo quadrado, a média na polarização do fóton e a soma sobre os spins do quark e do antiquark, obtemos

$$
\begin{aligned}
\left|\psi_{h, \bar{h}}^{T(\lambda=+)}\right|^{2} & =\frac{1}{2} \sum_{h} \sum_{\bar{h}} \sum_{f} 2 \frac{N_{C}}{4 \pi} 4 \pi \alpha_{e m} e_{f}^{2}\left\{\epsilon^{2}\left[z^{2} \delta_{h+} \delta_{\bar{h}-}+(1-z)^{2} \delta_{h-} \delta_{\bar{h}+}\right] \frac{K_{1}^{2}(\epsilon r)}{4 \pi^{2}}\right. \\
& \left.+m^{2} \delta_{h+} \delta_{\bar{h}+} \frac{K_{0}^{2}(\epsilon r)}{4 \pi^{2}}\right\} \\
& =\frac{1}{2} \frac{N_{C} \alpha_{e m}}{2 \pi^{2}} \sum_{f} e_{f}^{2}\left\{\epsilon^{2}\left[z^{2}+(1-z)^{2}\right] K_{1}^{2}(\epsilon r)+m^{2} K_{0}^{2}(\epsilon r)\right\}
\end{aligned}
$$

Além disso, o termo $\left|\psi_{h, \bar{h}}^{T(\lambda=-)}\right|^{2}$ produzirá a mesma expressão. Somando as contribuições para $\lambda= \pm 1$ temos

$$
\left|\psi_{h, \bar{h}}^{T}\right|^{2}=\frac{N_{C} \alpha_{e m}}{2 \pi^{2}} \sum_{f} e_{f}^{2}\left\{\epsilon^{2}\left[z^{2}+(1-z)^{2}\right] K_{1}^{2}(\epsilon r)+m^{2} K_{0}^{2}(\epsilon r)\right\} .
$$

Por fim, vamos fazer um breve comentário sobre os fatores de normalização de $1 / 4 \pi$ que foram incluídos nas funções de onda. Esse fator pode estar presente no espaço de fase do cone de luz, e portanto estar presente explicitamente na fórmula do formalismo de dipolo (como na Ref.[31]), ou pode estar absorvido diretamente nas funções de onda, que é a forma que utilizamos nessa tese.

\section{A.2 Funções de onda de mésons vetoriais}

As funções de onda do cone de luz para mésons vetoriais são construídas de forma análoga ao caso do fóton virtual em que calculamos anteriormente. Porém, nesse caso temos um estado ligado e não uma partícula fundamental e, portanto, não temos condições de obtê-la através de um cálculo de primeiros princípios da QCD.

Uma forma de modelar a função de onda do méson vetorial é assumindo que o méson vetorial é, predominantemente, um estado $q \bar{q}$ e que sua estrutura de spin é a mesma do fóton. Assim temos

$$
\begin{aligned}
\psi_{h \bar{h}}^{T(\lambda= \pm)}(\vec{r}, z) & = \pm \sqrt{2} \sqrt{\frac{N_{C}}{4 \pi}} \frac{1}{z(1-z)}\left\{i e^{ \pm i \theta}\left(z \delta_{h \pm} \delta_{\bar{h} \mp}-(1-z) \delta_{h \mp} \delta_{\bar{h} \pm}\right) \partial_{r}+m \delta_{h \pm} \delta_{\bar{h} \pm}\right\} \\
& \times \phi_{T}(r, z)
\end{aligned}
$$

$\mathrm{e}$

$$
\psi^{L}(z, r)=\sqrt{\frac{N_{C}}{4 \pi}} \delta_{h,-\bar{h}}\left[M_{V}+\delta \frac{m_{f}^{2}-\nabla_{r}^{2}}{M_{V} z(1-z)}\right] \phi_{L}(r, z),
$$

onde $\nabla_{r}^{2}=(1 / r) \partial_{r}+\partial_{r}^{2}$ e $M_{V}$ é a massa do méson. Além disso, nas equações acima, $\phi_{L, T}(r, z)$ são as partes escalares da função de onda do méson vetorial e são obtidas da função de onda do fóton pelas trocas

$$
e_{f} e z(1-z) \frac{K_{0}(\epsilon r)}{2 \pi} \rightarrow \phi_{T, L}(r, z)
$$




\begin{tabular}{||c|c|c|c|c|c|c|c||}
\hline \hline Meson & $M_{V} / \mathrm{GeV}$ & $m_{f} / \mathrm{GeV}$ & $e_{f}$ & $N_{T}$ & $R_{T}^{2} / \mathrm{GeV}^{-2}$ & $N_{L}$ & $R_{L}^{2} / \mathrm{GeV}^{-2}$ \\
\hline \hline$J / \psi$ & 3,097 & 1,4 & $2 / 3$ & 1,23 & 6.5 & 0,83 & 3,0 \\
\hline$\phi$ & 1,019 & 0,14 & $1 / 3$ & 4,75 & 16,0 & 1,41 & 9,7 \\
\hline$\rho$ & 0,776 & 0,14 & $1 / \sqrt{2}$ & 4,47 & 21,9 & 1,79 & 10,4 \\
\hline$\Upsilon$ & 9,460 & 4,5 & $1 / 3$ & 0,67 & 2,16 & 0,47 & 1,01 \\
\hline$\Upsilon$ & 9,460 & 4,2 & $1 / 3$ & 0,76 & 1,91 & 0,47 & 1,01 \\
\hline \hline
\end{tabular}

Tab. A.1: Parâmetros para a função de onda Gaus-LC.

\begin{tabular}{||c|c|c|c|c|c|c||}
\hline \hline Meson & $M_{V} / \mathrm{GeV}$ & $m_{f} / \mathrm{GeV}$ & $e_{f}$ & $\mathcal{N}_{T}$ & $\mathcal{N}_{L}$ & $\mathcal{R}^{2} / \mathrm{GeV}^{-2}$ \\
\hline \hline$J / \psi$ & 3,097 & 1,4 & $2 / 3$ & 0,578 & 0,575 & 2,3 \\
\hline$\phi$ & 1,019 & 0,14 & $1 / 3$ & 0,919 & 0,825 & 11,2 \\
\hline$\rho$ & 0,776 & 0,14 & $1 / \sqrt{2}$ & 0,911 & 0,853 & 12,9 \\
\hline$\Upsilon$ & 9,460 & 4,5 & $1 / 3$ & 0,469 & 0,469 & 0,55 \\
\hline$\Upsilon$ & 9,460 & 4,2 & $1 / 3$ & 0,481 & 0,480 & 0,57 \\
\hline \hline
\end{tabular}

Tab. A.2: Parâmetros para a função de onda Boosted Gaussian.

$\mathrm{e}$

$$
2 Q \rightarrow M_{V}
$$

Existem modelos para essas funções onda dos quais utilizamos o "Gaus-LC" e o "BoostedGaussian" [31].

Outro comentário importante é sobre o fator $\delta$ que surge na Eq.(A.42). Esse fator é um fator não local (que não existe no caso de fótons) e que depende do modelo usado. Para os modelos usados nessa tese, temos $\delta=0$ para Gaus-LC e $\delta=1$ para Boosted Gaussian.

Em nossos cálculos estamos interessados na produção exclusiva de mésons vetoriais, onde no estado inicial temos um fóton virtual o qual flutua em um dipolo de cor que se converte em um méson vetorial no estado final. No formalismo de dipolo isso é descrito pelo chamado overlap entre as funções de onda do fóton virtual e do méson vetorial, que é dado por

$$
\left(\Psi_{V}^{*} \Psi\right)_{T}=e_{f} e \frac{N_{c}}{\pi z(1-z)}\left\{m_{f}^{2} K_{0}(\epsilon r) \phi_{T}(r, z)-\left[z^{2}+(1-z)^{2}\right] \epsilon K_{1}(\epsilon r) \partial_{r} \phi_{T}(r, z)\right\},
$$

para a polarização transversa e

$$
\left(\Psi_{V}^{*} \Psi\right)_{L}=e_{f} e \frac{N_{c}}{4 \pi^{2}} 2 Q z(1-z) K_{0}(\epsilon r)\left[M_{V} \phi_{L}(r, z)+\delta \frac{m_{f}^{2}-\nabla_{r}^{2}}{M_{V} z(1-z)} \phi_{L}(r, z)\right]
$$

para a polarização longitudinal.

Vamos agora explicitar os modelos para $\phi_{T, L}$ usados nessa tese. Para o modelo Gaus-LC usamos

$$
\phi_{T}(r, z)=N_{T}[z(1-z)]^{2} \exp \left(-r^{2} / 2 R_{T}^{2}\right)
$$


$\mathrm{e}$

$$
\phi_{L}(r, z)=N_{L} z(1-z) \exp \left(-r^{2} / 2 R_{L}^{2}\right) .
$$

Já, para o modelo Boosted Gaussian usamos

$$
\phi_{T, L}(r, z)=\mathcal{N}_{T, L} z(1-z) \exp \left(-\frac{m_{f}^{2} \mathcal{R}^{2}}{8 z(1-z)}-\frac{2 z(1-z) r^{2}}{\mathcal{R}^{2}}+\frac{m_{f}^{2} \mathcal{R}^{2}}{2}\right) .
$$

As constantes $R_{T, L}$ e $N_{T, L}$ do modelo Gaus-LC, bem como, as constantes $\mathcal{N}_{T, L}$ e $\mathcal{R}$ do modelo Boosted Gaussian são obtidas através de condições de normalização impostas à função de onda do méson vetorial (para maiores detalhes ver Ref.[31]), e os valores podem ser encontrados nas Refs.[2, 31] e são apresentados nas Tabs.A.1 e A.2. 


\section{Apêndice B}

\section{Soluções da Equação BK}

Nessa seção, vamos apresentar algumas soluções analíticas aproximadas da equação BK, válidas para certos regimes cinemáticos. Para isso, vamos seguir as referências $[9,42]$

\section{B.1 Soluções fora da região de saturação}

Para dipolos muito pequenos, a amplitude de espalhamento é pequena e os efeitos de saturação não são importantes. Dessa forma temos $\mathcal{N} \ll 1$.

Então para $\mathcal{N} \ll 1$, a equação BK pode ser linearizada $\left(\bar{\alpha}_{s}=\frac{\alpha_{s} N_{c}}{\pi}\right)$

$$
\frac{\partial \mathcal{N}\left(x_{10}, Y\right)}{\partial Y}=\frac{\bar{\alpha}_{s}}{2 \pi} \int \mathrm{d} x_{2} \frac{x_{10}^{2}}{x_{20}^{2} x_{21}^{2}}\left[\mathcal{N}\left(x_{12}, Y\right)+\mathcal{N}\left(x_{20}, Y\right)-\mathcal{N}\left(x_{10}, Y\right)\right] .
$$

A equação acima é exatamente a equação BFKL no espaço das posições [9, 18].

Vamos partir da solução formal da equação acima (maiores detalhes podem ser encontrados nas Refs.[9, 42])

$$
\begin{aligned}
\mathcal{N}\left(x_{\perp}, Y\right) & =\int \mathrm{d} \nu e^{\bar{\alpha}_{s} Y \chi(0, \nu)}\left(x_{\perp} Q_{s 0}\right)^{1+2 i \nu} C_{\nu} \\
& =\int \mathrm{d} \nu C_{\nu} \exp \left\{\bar{\alpha}_{s} Y \chi(0, \nu)+(1+2 i \nu) \ln \left(x_{\perp} Q_{s 0}\right)\right\}
\end{aligned}
$$

onde $Q_{s 0}$ é uma escala transversa inicial.

\section{B.1.1 Aproximação de Difusão}

Vamos considerar a aproximação de difusão que corresponde a tomar $x_{\perp} \sim \rho\left(\equiv 1 / Q_{s 0}\right)$. Inicialmente, temos

$$
\begin{aligned}
\mathcal{N}\left(x_{\perp}, Y\right) & =\int_{-\infty}^{\infty} \mathrm{d} \nu C_{\nu} x_{\perp}^{1+2 i \nu} \rho^{-1-2 i \nu} e^{\bar{\alpha}_{s} \chi(0, \nu) Y} \\
& =\frac{x_{\perp}}{\rho} \int_{-\infty}^{\infty} \mathrm{d} \nu C_{\nu} \exp \left\{\bar{\alpha}_{s} \chi(0, \nu) Y+\ln \left(\frac{x_{\perp}}{\rho}\right)^{2 i \nu}\right\} \\
& =\frac{x_{\perp}}{\rho} \int_{-\infty}^{\infty} \mathrm{d} \nu C_{\nu} \exp \left\{\bar{\alpha}_{s} \chi(0, \nu) Y+2 i \nu \ln \left(\frac{x_{\perp}}{\rho}\right)\right\}
\end{aligned}
$$


Vamos calcular essa integral usando o método do ponto de sela. Como $x_{\perp} \sim \rho$, temos que $\ln \left(x_{\perp} / \rho\right) \sim 0$ e portanto o ponto de sela do integrando será o ponto de sela de $\chi(0, \nu)$ o qual ocorre em $\nu_{D}=0$. Expandindo $\chi(0, \nu)$ em torno do ponto de sela, obtemos [42]

$$
\chi(0, \nu) \approx 4 \ln 2-14 \zeta(3) \nu^{2}
$$

sendo $\zeta$ a função zeta de Riemann.

Dessa forma a amplitude $\mathcal{N}$ fica

$$
\begin{aligned}
\mathcal{N} & \approx \frac{x_{\perp}}{\rho} C_{\nu_{D}} \int_{-\infty}^{\infty} \mathrm{d} \nu \exp \left\{\bar{\alpha}_{s} Y\left[4 \ln 2-14 \zeta(3) \nu^{2}\right]+2 i \nu \ln \left(\frac{x_{\perp}}{\rho}\right)\right\} \\
& =\frac{x_{\perp}}{\rho} C_{\nu_{D}} e^{(4 \ln 2) \bar{\alpha}_{s} Y} \int_{-\infty}^{\infty} \mathrm{d} \nu \exp \left\{-14 \bar{\alpha}_{s} Y \zeta(3) \nu^{2}+i \nu \ln \left(\frac{x_{\perp}}{\rho}\right)^{2}\right\}
\end{aligned}
$$

Podemos identificar o termo entre chaves da equação acima como um termo do tipo $a^{2}+$ $2 a b\left(=(a+b)^{2}-b^{2}\right)$, com

$$
\begin{aligned}
a^{2} & =-14 \bar{\alpha}_{s} Y \zeta(3) \nu^{2} \rightarrow a=i \nu \sqrt{14 \bar{\alpha}_{s} Y \zeta(3)} \\
2 a b & =2 i \nu \ln \left(\frac{x_{\perp}}{\rho}\right) \rightarrow b=\frac{2 i \nu \ln \left(\frac{x_{\perp}}{\rho}\right)}{2 i \nu \sqrt{14 \bar{\alpha}_{s} Y \zeta(3)}}=\frac{\ln \left(\frac{x_{\perp}}{\rho}\right)}{\sqrt{14 \bar{\alpha}_{s} Y \zeta(3)}} \\
\rightarrow b^{2} & =\frac{\ln ^{2}\left(\frac{x_{\perp}}{\rho}\right)}{14 \bar{\alpha}_{s} Y \zeta(3)} .
\end{aligned}
$$

Sendo assim, fazendo a troca $a^{2}+2 a b \rightarrow(a+b)^{2}-b^{2}$ no argumento da exponencial, obtemos

$$
\begin{aligned}
\mathcal{N} & =\frac{x_{\perp}}{\rho} C_{\nu_{D}} e^{(4 \ln 2) \bar{\alpha}_{s} Y} \exp \left\{-\frac{\ln ^{2}\left(x_{\perp} / \rho\right)}{14 \bar{\alpha}_{s} Y \zeta(3)}\right\} \\
& \left.\times \int_{-\infty}^{\infty} \mathrm{d} \nu \exp \left\{i\left(\nu \sqrt{14 \bar{\alpha}_{s} Y \zeta(3)}-\frac{i \ln \left(\frac{x_{\perp}}{\rho}\right)}{\sqrt{14 \bar{\alpha}_{s} Y \zeta(3)}}\right)\right]^{2}\right\} .
\end{aligned}
$$

Efetuando a seguinte troca de variáveis

$$
\begin{aligned}
u & =\nu \sqrt{14 \bar{\alpha}_{s} Y \zeta(3)}-\frac{i \ln \left(\frac{x_{\perp}}{\rho}\right)}{\sqrt{14 \bar{\alpha}_{s} Y \zeta(3)}} \\
\rightarrow \mathrm{d} u & =\sqrt{14 \bar{\alpha}_{s} Y \zeta(3)} \mathrm{d} \nu
\end{aligned}
$$

a integral em $\nu$ pode ser reescrita como

$$
\frac{1}{\sqrt{14 \bar{\alpha}_{s} Y \zeta(3)}} \int_{-\infty}^{\infty} \mathrm{d} u e^{-u^{2}}=\frac{\sqrt{\pi}}{\sqrt{14 \bar{\alpha}_{s} Y \zeta(3)}}
$$

Juntando todos os resultados, a amplitude para a aproximação de difusão é dada por

$$
\mathcal{N}=\frac{x_{\perp}}{\rho} C_{\nu_{D}} \sqrt{\frac{\pi}{14 \bar{\alpha}_{s} Y \zeta(3)}} \exp \left\{\left(\alpha_{\mathbb{P}}-1\right) Y-\frac{\ln ^{2}\left(x_{\perp} / \rho\right)}{14 \bar{\alpha}_{s} Y \zeta(3)}\right\},
$$

onde $\alpha_{\mathbb{P}}-1=4 \bar{\alpha}_{s} \ln 2$ é o intercept do Pomeron perturbativo (Pomeron BFKL). 


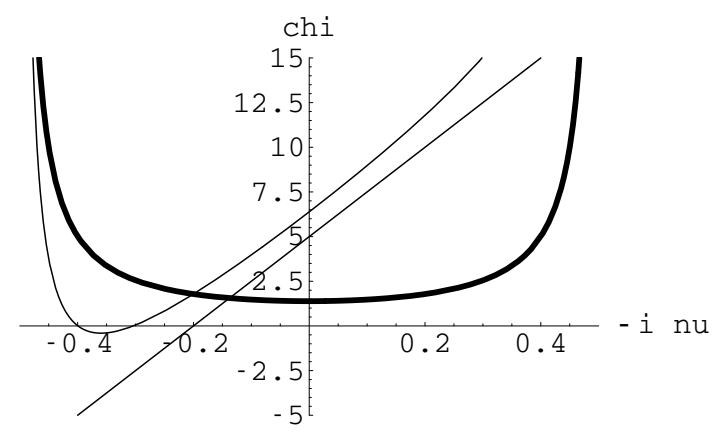

Fig. B.1: Comportamento da função característica da BFKL [42].

\section{B.1.2 Aproximação de Duplo Logaritmo Dominante}

Vamos estudar o limite de dipolos muito pequenos $x_{\perp} Q_{s 0} \ll 1$ (isto é, $Q^{2}$ muito grande), que corresponde à aproximação de duplo logaritmo dominante ("Double Logarithm Approximation" - DLA).

Se não existisse o termo logaritmico na Eq.(B.2), teríamos um ponto de sela em $\nu=0$. Entretanto, o termo logaritmico com argumento muito menor que um, desloca o ponto de sela em direção à $\nu=-i / 2$ (conforme mostra a Fig.B.1). Expandindo $\chi(0, \nu)$ em torno de $\nu=-i / 2$, temos $[9,42]$

$$
\chi(0, \nu)=\frac{2}{1-2 i \nu} .
$$

A Eq.(B.2) pode ser reescrita como

$$
\mathcal{N}\left(x_{\perp}, Y\right)=\int_{-\infty}^{\infty} \mathrm{d} \nu C_{\nu} e^{F(\nu)}
$$

onde definimos

$$
F(\nu)=a \chi(0, \nu)+(1+2 i \nu) \ln r
$$

$\mathrm{e}$

$$
a=\bar{\alpha}_{s} Y, r=x_{\perp} Q_{s 0} .
$$

Para resolver a Eq.(B.11), vamos utilizar o método do ponto de sela. Impondo que $\mathrm{d} F / \mathrm{d} \nu=0$, encontramos o ponto de sela $\nu^{*}$

$$
\nu^{*} \approx-\frac{i}{2}\left(1-\sqrt{\frac{2 \bar{\alpha}_{s} Y}{\ln \left(1 / x_{\perp} Q_{s 0}\right)}}\right) .
$$


Até segunda ordem em $\nu-\nu^{*}$, podemos escrever

$$
F(\nu) \approx F\left(\nu^{*}\right)+\left(\nu-\nu^{*}\right) \frac{\mathrm{d} F\left(\nu^{*}\right)}{\mathrm{d} \nu}+\frac{\left(\nu-\nu^{*}\right)^{2}}{2} \frac{\mathrm{d}^{2} F\left(\nu^{*}\right)}{\mathrm{d} \nu^{2}} .
$$

Da forma como definimos $F$, temos

$$
\frac{\mathrm{d}^{2} F}{\mathrm{~d} \nu^{2}}=a \frac{\mathrm{d}^{2} \chi(0, \nu)}{\mathrm{d} \nu^{2}}=a \chi^{\prime \prime}(0 . \nu)
$$

Com isso, a amplitude pode ser escrita como

$$
\mathcal{N}=C_{\nu^{*}} e^{F\left(\nu^{*}\right)} \int_{-\infty}^{\infty} \mathrm{d} \nu e^{\left(a \chi^{\prime \prime}\left(0, \nu^{*}\right) / 2\right)\left(\nu-\nu^{*}\right)^{2}}
$$

onde

$$
F\left(\nu^{*}\right)=\ln r^{2}+2 \sqrt{2 a \ln (1 / r)} .
$$

Fazendo uma troca de variáveis adequada, a integral que surge na Rq.(B.17) pode ser escrita como

$$
\int_{-\infty}^{\infty} \mathrm{d} \nu e^{\left(a \chi^{\prime \prime}\left(0, \nu^{*}\right) / 2\right) \nu^{2}}=\sqrt{\frac{-2 \pi}{a \chi^{\prime \prime}\left(0, \nu^{*}\right)}} .
$$

No ponto de sela, a derivada segunda da função característica fica

$$
\begin{aligned}
\chi^{\prime \prime}\left(0, \nu^{*}\right) & =-\frac{16}{\left(1-2 i \nu^{*}\right)^{3}} \\
& =\frac{-16}{(2 a)^{3 / 2}} \ln ^{3 / 2}(1 / r) .
\end{aligned}
$$

Juntando esses resultados (e substituindo $a$ e $r$ pelas variáveis originais), obtemos a amplitude de espalhamento em DLA

$$
\mathcal{N}=\left(x_{\perp} Q_{s 0}\right)^{2} C_{\nu^{*}} \frac{\sqrt{\pi}}{2}\left(2 \bar{\alpha}_{s} Y\right)^{1 / 4} \ln ^{-3 / 4}\left(\frac{1}{x_{\perp} Q_{s 0}}\right) \exp \left\{2 \sqrt{2 \bar{\alpha}_{s} Y \ln \left(\frac{1}{x_{\perp} Q_{s 0}}\right)}\right\} .
$$

Observamos que $\mathcal{N}$ cresce com a rapidez e para altas energias, violará o limite de disco negro. Portanto, esse resultado é válido para baixas energias (longe da região de saturação).

\section{B.1.3 Solução próximo ao regime de saturação}

Vamos agora estudar a região onde o tamanho do dipolo ainda é pequeno (portanto fora da saturação), mas não muito menor do que $Q_{s 0}\left(x_{\perp} Q_{s 0} \lesssim 1\right)$. Como essa região ainda é linear, podemos usar a Eq.(B.2).

A localização do ponto de sela $\left(\nu_{s p}\right)$ é determinada por

$$
\bar{\alpha}_{s} \chi^{\prime}\left(0, \nu_{s p}\right) Y+2 i \ln \left(x_{\perp} Q_{s 0}\right)=0 .
$$


Vamos aproximar a integral em $\nu$ (na Eq.(B.2)) pelo valor do integrando no ponto de sela

$$
\mathcal{N}\left(x_{\perp}, Y\right) \propto\left(x_{\perp} Q_{s 0}\right)^{1+2 i \nu_{s p}} e^{\bar{\alpha}_{s} \chi\left(0, \nu_{s p}\right) Y}
$$

A amplitude dada pela Eq.(B.23) cresce com a energia e com o tamanho do dipolo. Quando $\mathcal{N} \sim 1$, essa aproximação quebra e devemos usar a equação BK. Vamos estudar a região onde ocorre essa quebra do regime linear. Queremos encontrar uma linha no plano $\left(x_{\perp}, Y\right)$ ao longo da qual $\mathcal{N} \approx 1$. Isso irá nos fornecer a escala de saturação.

A escala de saturação $Q_{s}=Q_{s}(Y)$ é definida pela condição

$$
\mathcal{N}\left(x_{\perp}=1 / Q_{s}(Y), Y\right)=\text { constante }
$$

onde a constante é da ordem de 1. Usando a Eq.(B.23) na equação acima, obtemos

$$
1=\left(\frac{Q_{s 0}}{Q_{s}(Y)}\right)^{1+2 i \nu_{0}} e^{\bar{\alpha}_{s} \chi\left(0, \nu_{0}\right) Y}
$$

levando a

$$
\bar{\alpha}_{s} \chi\left(0, \nu_{0}\right) Y+\left(1+2 i \nu_{0}\right) \ln \left(\frac{Q_{s 0}}{Q_{s}(Y)}\right)=0
$$

com $\nu_{0}=\nu_{s p}$. Tomando a condição de ponto de sela (Eq.(B.22)) para $x_{\perp}=1 / Q_{s}(Y)$ ao longo da linha de saturação

$$
\bar{\alpha}_{s} \chi^{\prime}\left(0, \nu_{0}\right) Y+2 i \ln \left(\frac{Q_{s 0}}{Q_{s}(Y)}\right)=0 .
$$

Da Eq.(B.26), podemos escrever

$$
Q_{s}(Y)=Q_{s 0} \exp \left\{\bar{\alpha}_{s} \frac{\chi\left(0, \nu_{0}\right)}{1+2 i \nu_{0}} Y\right\} .
$$

Usando as Eqs.(B.26) e (B.27), obtemos

$$
\frac{\chi^{\prime}\left(0, \nu_{0}\right)}{\chi\left(0, \nu_{0}\right)}=\frac{2 i}{1+2 i \nu_{0}} .
$$

A equação acima pode ser resolvida numericamente, fornecendo

$$
\nu_{0} \approx-0.1275 i
$$

Usando esse valor na Eq.(B.28), temos

$$
Q_{s}(Y) \approx Q_{s 0} e^{2.44 \bar{\alpha}_{s} Y} .
$$

Observamos que a escala de saturação cresce com uma exponencial em $Y$. 
Vamos eliminar a dependência em $Y$ da Eq.(B.23). Usando a Eq.(B.28)

$$
\bar{\alpha}_{s} Y=\ln \left(\frac{Q_{s}(Y)}{Q_{s 0}}\right)^{\frac{\left(1+2 i \nu_{0}\right)}{\chi\left(0, \nu_{0}\right)}}
$$

de forma que a Eq.(B.23) fica

$$
\mathcal{N}\left(x_{\perp}, Y\right) \propto\left(x_{\perp} Q_{s 0}\right)^{1+2 i \nu_{s p}}\left(\frac{Q_{s}(Y)}{Q_{s 0}}\right)^{\left(1+2 i \nu_{0}\right) \frac{\chi\left(0, \nu_{s p}\right)}{\chi\left(0, \nu_{0}\right)}}
$$

Como $\nu_{s p}=\nu_{0}$, obtemos $[121,122]$

$$
\mathcal{N}\left(x_{\perp}, Y\right) \propto\left[x_{\perp} Q_{s}(Y)\right]^{1+2 i \nu_{0}} .
$$

Em geral, deveríamos esperar que $\mathcal{N}\left(x_{\perp}, Y\right)$ fosse função de duas variáveis independentes. Mas pela Eq.(B.33), vemos que a amplitude escala com o produto $x_{\perp} Q_{s}(Y)$. Esse scaling observado fora da região de saturação é o chamado scaling geométrico extendido.

\section{B.2 Solução dentro da região de saturação}

Dentro da região de saturação, quando os dipolos são grandes (isto é $1 / \Lambda_{Q C D} \gg x_{\perp} \gg$ $\left.1 / Q_{s}(Y)\right)$ o limite de disco negro $(\mathcal{N}=1)$ é alcançado.

Temos que

$$
S=1-\mathcal{N}
$$

e portanto no limite de disco negro

$$
S \ll 1
$$

A equação BK em termos de $S$ no limite de disco negro (onde o termo não linear pode ser desprezado) fica

$$
\frac{\partial S\left(x_{10}, Y\right)}{\partial Y}=-\frac{\alpha_{s} N_{c}}{2 \pi^{2}} \int \mathrm{d}^{2} x_{2} \frac{x_{10}^{2}}{x_{20}^{2} x_{21}^{2}} S\left(x_{10}, Y\right) .
$$

Temos a seguinte relação $[9,123]$

$$
\int \mathrm{d}^{2} x_{2} \frac{x_{10}^{2}}{x_{20}^{2} x_{21}^{2}}=4 \ln \frac{x_{10}}{\rho}
$$

onde $\rho$ é um corte que evita as divergências para $x_{20}$ e $x_{21}$ pequenos. Tomando $\rho=1 / Q_{s}(y)$, a equação BK pode ser escrita como

$$
\frac{\partial S\left(x_{10}, Y\right)}{\partial Y}=-\bar{\alpha}_{s} \ln \left[x_{10} Q_{s}(Y)\right]^{2} S\left(x_{10}, Y\right)
$$


Motivados pela equação acima e pelo cálculo da subseção anterior, vamos definir a varável de scaling

$$
\xi=\ln \left[x_{\perp} Q_{s}(Y)\right]^{2}
$$

onde $x_{\perp}=x_{10}$. Temos que

$$
\frac{\partial \xi}{\partial Y}=2\left[x_{\perp}^{2} Q_{s}^{2}\right]^{-1} x_{\perp}^{2} Q_{s}(Y) \frac{\partial Q_{s}(y)}{\partial Y}
$$

e usando a Eq.(B.28), podemos escrever

$$
\frac{\partial Q_{s}(y)}{\partial Y}=\frac{\bar{\alpha}_{s} \chi\left(0, \nu_{0}\right)}{1+2 i \nu_{0}} Q_{s}(Y)
$$

de forma que

$$
\begin{aligned}
\frac{\partial \xi}{\partial Y} & =\frac{\partial \ln \left[x_{\perp}^{2} Q_{s}^{2}(Y)\right]}{\partial Y} \\
& =\frac{2 \chi\left(0, \nu_{0}\right)}{1+2 i \nu_{0}} \bar{\alpha}_{s} .
\end{aligned}
$$

Usando a Eq.(B.40) juntamente com a equação acima, obtemos a seguinte equação diferencial

$$
\frac{\partial S(\xi)}{\partial Y}=-\frac{1+2 i \nu_{0}}{2 \chi\left(0, \nu_{0}\right)} \xi S(\xi)
$$

cuja solução é

$$
S(\xi)=S_{0} \exp \left\{-\frac{1+2 i \nu_{0}}{2 \chi\left(0, \nu_{0}\right)} \frac{\xi^{2}}{2}\right\}
$$

onde $S_{0}$ é uma constante menor do que 1. A amplitude de dipolo correspondente é

$$
\mathcal{N}(\xi)=1-S_{0} \exp \left\{-\frac{1+2 i \nu_{0}}{2 \chi\left(0, \nu_{0}\right)} \frac{\xi^{2}}{2}\right\} .
$$

A equação acima é conhecida como fórmula de Levin e Tuchin [123]. Novamente, a solução acima é dada em termos de uma única variável $(\xi)$. Esse é o scaling geométrico. 


\section{Apêndice $\mathrm{C}$}

\section{Aproximação de Fótons Equivalentes}

\section{C.1 Derivação do espectro de fótons equivalentes}

Nesse apêndice vamos derivar o espectro de fótons equivalentes através da QED. Em nossos cálculos, vamos seguir a Ref.[62]. Consideremos os dois tipos de processos, mostrados na Fig.C.1: (a) colisão entre um fóton real $\left(k^{2}=0\right)$ e uma partícula com quadrimomentum $q\left(q^{2}=m^{2}\right)$, cujo sistema resultante é uma partícula ou um conjunto de partículas com quadrimomentum $Q$; (b) colisão entre a mesma partícula de quadrimomentum $q$ e outra partícula de quadrimomentum $p$ e massa $M\left(p^{2}=M^{2}\right)$. Após a colisão, a última partícula tem quadrimomentum $p^{\prime}$ e o mesmo sistema com quadrimomentum $Q$ é formado. Esse processo pode ser considerado como uma colisão entre a partícula de quadrimomentum $q$ e um fóton virtual emitido pela partícula de quadrimomentum $p$. Esse fóton tem quadrimomentum $k=p-p^{\prime}\left(k^{2}<0\right)$. Se $|k|^{2}$ é pequeno, o fóton virtual não é muito diferente de um fóton real. Tal situação é possível em colisões de partículas muito rápidas: o campo eletromagnético de uma partícula carregada com velocidade $v \approx 1$ é praticamente transverso ao seu movimento e, portanto, tem propriedades similares àquelas do campo de uma onda de luz. Nessas condições a seção de choque do processo (b) pode ser expressa em termos da seção de choque do processo (a).

Vamos supor que a partícula de massa $M$ é ultra-relativística, com energia (no referencial de repouso da partícula de massa $m) \epsilon \gg M$.

A amplitude do processo (a) (onde o fóton é real) pode ser escrita como

$$
M_{f i}^{r}=-e e_{\mu} J^{\mu}
$$

onde $e_{\mu}$ é o quadrivetor polarização do fóton e $J_{\mu}$ é a corrente de transição correspondente ao vértice no diagrama. A amplitude do processo (b) é

$$
M_{f i}=Z e^{2} \frac{1}{k^{2}} j_{\mu} J^{\mu},
$$

sendo $j_{\mu}$ a corrente de transição da partícula de massa $M$ (vértive inferior), $Z e$ é a carga dessa partícula e $J_{\mu}$ é uma função de $k=Q-q$ e portanto é diferente do caso anterior onde $k^{2}=0$. Se no segundo caso

$$
|k|^{2} \ll m^{2}
$$




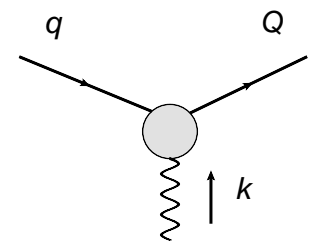

(a)

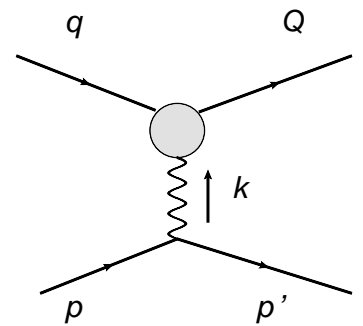

(b)

Fig. C.1: (a) Colisão entre um fóton real e uma partícula de quadrimomentum q e (b) uma partícula de quadrimomentum inicial p emite um fóton virtual que interage com uma partícula de quadrimomentum q. Nos dois casos é gerado um estado final com quadrimomentum $Q$.

podemos tomar $J$ para $k^{2}=0$.

Se a mudança no momentum da partícula de massa $M$, quando um fóton virtual é emitido $\left(\vec{p}-\vec{p}^{\prime}=\vec{k}\right)$, é pequena comparada com $|\vec{p}| \approx \epsilon$ (momentum inicial), podemos tomar $\vec{p}=\vec{p}^{\prime}$ na corrente de transição $j$. O movimento da partícula $M$ pode ser considerado como um movimento uniforme em linha reta. Como tal movimento é quase clássico, a corrente correspondente é independente do spin da partícula:

$$
j^{\mu}=2 p^{\mu} .
$$

Considerando que o movimento ocorre no eixo $x\left(\vec{p}=p_{x} \hat{i}\right)$ e usando a condição de que a corrente seja transversa, obtemos

$$
\begin{aligned}
j^{\mu} k_{\mu} & =2 \epsilon \omega-2 p_{x} k_{x}=0 \\
\rightarrow \omega & =\frac{p_{x}}{\epsilon} k_{x}=v k_{x} .
\end{aligned}
$$

Portanto

$$
\begin{aligned}
-k^{2} & =-\omega^{2}+k_{x}^{2}+\vec{k}_{\perp}^{2}=-\omega^{2}+\frac{\omega^{2}}{v^{2}}+\vec{k}_{\perp}^{2}= \\
& =\frac{\omega^{2}}{v^{2}}\left(1-v^{2}\right)+\vec{k}_{\perp}^{2} \approx \omega^{2}\left(1-v^{2}\right)+\vec{k}_{\perp}^{2},
\end{aligned}
$$

onde $\vec{k}_{\perp}=k_{y} \hat{j}+k_{z} \hat{k}$. Vemos que a condição (C.3) é equivalente a $\left|\vec{k}_{\perp}\right| \ll m$ e $\omega \ll$ $m / \sqrt{1-v^{2}}=\gamma m$.

Da condição de que a corrente seja transversa $\left(J^{\mu} k_{\mu}=0\right)$, temos (usando a Eq.(C.5), $k^{\mu}=(\omega, \vec{k})$ e $\left.J^{\mu}=\left(J_{0}, \vec{J}\right)\right)$

$$
J^{\mu} k_{\mu}=J_{0} \omega-J_{x} k_{x}-\vec{J}_{\perp} \cdot \vec{k}_{\perp}=0
$$




$$
\rightarrow J_{0}=\frac{J_{x}}{v}+\frac{\vec{J}_{\perp} \cdot \vec{k}_{\perp}}{\omega}
$$

Portanto, obtemos para $j_{\mu} J^{\mu}$

$$
\begin{aligned}
j_{\mu} J^{\mu} & =2\left(\epsilon, p_{x} \hat{i}\right) \cdot\left(J_{0}, \vec{J}\right) \\
& =2\left\{\epsilon\left[\frac{J_{x}}{v}+\frac{\vec{J}_{\perp} \cdot \vec{k}_{\perp}}{\omega}\right]-p_{x} J_{x}\right\} \\
& =2\left\{\frac{\left(\epsilon^{2}-p_{x}^{2}\right)}{p_{x}} J_{x}+\epsilon \frac{\vec{J}_{\perp} \cdot \vec{k}_{\perp}}{\omega}\right\},
\end{aligned}
$$

onde na última linha usamos $v=p_{x} / \epsilon$. Tomando $p_{x} \approx \epsilon$ e usando $\epsilon^{2}-p_{x}^{2}=M^{2}$

$$
j_{\mu} J^{\mu} \approx 2 \frac{\epsilon}{\omega}\left(\vec{k}_{\perp} \cdot \vec{J}_{\perp}+\frac{\omega M^{2}}{\epsilon^{2}} J_{x}\right) .
$$

O produto $J \cdot e$ na Eq.(C.1) pode ser feito no gauge transverso em 3 dimensões, onde $e^{\mu}=(0, \vec{e})$ e $e^{\mu} k_{\mu}=-\vec{e} \cdot \vec{k}=0$. Dessa forma obtemos

$$
e_{x}=-\frac{\vec{e}_{\perp} \cdot \vec{k}_{\perp}}{k_{x}} \approx-\frac{\vec{e}_{\perp} \cdot \vec{k}_{\perp}}{\omega}
$$

usando $\omega=v k_{x}$ e $v \approx 1$ no último sinal de igualdade. Logo

$$
\begin{aligned}
J^{\mu} e_{\mu} & =\left(J_{0}, \vec{J}\right) \cdot(0, \vec{e}) \\
& =-\left(e_{x} J_{x}+\vec{e}_{\perp} \cdot \vec{J}_{\perp}\right) \\
& =-\vec{e}_{\perp} \cdot\left(\vec{J}_{\perp}-\frac{\vec{k}_{\perp}}{\omega} J_{x}\right) .
\end{aligned}
$$

As expressões (C.8) e (C.10) são proporcionais se os segundos termos de cada uma delas forem desprezíveis. Como a corrente $J$ pertence ao vértice superior do diagrama (b), ela não depende da direção de $\vec{p}$; portanto $J_{x}$ e $\overrightarrow{J_{\perp}}$ devem ser quantidades da mesma ordem. Para que os termos em questão sejam negligenciáveis, devemos ter $\left|\vec{k}_{\perp}\right| \ll \omega$ e $\omega \ll \epsilon^{2}\left|\vec{k}_{\perp}\right| / M^{2}$. Essas condições são compatíveis com as condições anteriormente impostas em $\vec{k}_{\perp}$ e $\omega$.

Assumindo na Eq.(C.10) que o fóton é polarizado no plano de $\vec{x}$ e $\vec{k}$, temos $\vec{e}_{\perp} \| \vec{k}_{\perp}$. Além disso, a condição $\left|\vec{k}_{\perp}\right| \ll \omega$ implica que $\vec{e}_{\perp}^{2} \approx \vec{e}^{2}=1$ (vetor unitário), pois o 4-vetor $e^{\mu}$ satisfaz

$$
\begin{aligned}
e^{\mu *} e_{\mu} & =-1 \\
\rightarrow(0)^{2}-|\vec{e}|^{2} & =-1 \\
\rightarrow|\vec{e}|^{2} & =1 .
\end{aligned}
$$

Essas informações nos permitem reescrever as amplitudes como

$$
M_{f i}^{r}=-e\left[-\vec{e}_{\perp} \cdot \vec{J}_{\perp}\right]
$$


$\mathrm{e}$

$$
\begin{aligned}
M_{f i} & =Z e^{2} \frac{1}{k^{2}}\left[2 \frac{\epsilon}{\omega} \vec{k}_{\perp} \cdot \vec{J}_{\perp}\right] \\
& =e \frac{Z e}{k^{2}} 2 \frac{\epsilon}{\omega} \vec{k}_{\perp} \cdot \vec{J}_{\perp},
\end{aligned}
$$

mas

$$
\begin{aligned}
& \hat{k}_{\perp}=\vec{e}_{\perp}=\frac{\vec{k}_{\perp}}{\left|\vec{k}_{\perp}\right|} \\
& \vec{k}_{\perp}=\vec{e}_{\perp}\left|\vec{k}_{\perp}\right|
\end{aligned}
$$

$\log 0$

$$
\begin{aligned}
M_{f i} & =e \vec{e}_{\perp} \cdot \vec{J}_{\perp} \frac{Z e}{k^{2}} 2 \frac{\epsilon}{\omega}\left|\vec{k}_{\perp}\right| \\
& =M_{f i}^{r} \frac{Z e}{-k^{2}} 2 \frac{\epsilon}{\omega}\left|\vec{k}_{\perp}\right| .
\end{aligned}
$$

De acordo com a discussão prévia, as seguintes condições aqui assumidas são satisfeitas

$$
\begin{aligned}
\left|\vec{k}_{\perp}\right| & \ll \omega \ll m \gamma, \\
\frac{\omega}{\gamma^{2}} & \ll\left|\vec{k}_{\perp}\right| \ll m
\end{aligned}
$$

com a notação $\gamma=\epsilon / M=1 / \sqrt{1-v^{2}}$.

Disso, podemos encontrar a relação entre as correspondentes seções de choque. As seções de choque para os dois casos podem ser escritas como [18]

$$
\begin{aligned}
\mathrm{d} \sigma_{r} & =\left|M_{f i}^{r}\right|^{2}(2 \pi)^{4} \delta^{4}\left(P_{f}-P_{i}\right) \frac{1}{4 m \omega} \mathrm{d} \rho_{Q} \\
\mathrm{~d} \sigma & =\left|M_{f i}\right|^{2}(2 \pi)^{4} \delta^{4}\left(P_{f}-P_{i}\right) \frac{1}{4 m \epsilon} \frac{\mathrm{d}^{3} p^{\prime}}{2 \epsilon(2 \pi)^{3}} \mathrm{~d} \rho_{Q},
\end{aligned}
$$

onde $\mathrm{d} \rho_{Q}$ representa o espaço de fase do sistema de partículas $Q$. Além disso, para o caso com fóton real, temos $P_{f}-P_{i}=Q-k-q$ e para o caso com fóton virtual $P_{f}-P_{i}=Q+p^{\prime}-p-q$, com $-k=p^{\prime}-p$. Portanto, no caso em que o fóton virtual tem $k^{2} \approx 0$, as duas funções delta das seções de choque acima são iguais. Usando as Eqs.(C.6) e (C.13), obtemos

$$
\left|M_{f i}\right|^{2}=\left|M_{f i}^{r}\right|^{2} \frac{Z^{2} e^{2}}{\left(-k^{2}\right)^{2}} \frac{4 \epsilon^{2}}{\omega^{2}} \vec{k}_{\perp}^{2}
$$

com

$$
\left(-k^{2}\right)^{2}=\left[\frac{\omega^{2}}{\gamma^{2}}+\vec{k}_{\perp}^{2}\right]^{2} .
$$


Levando as duas expressões acima na Eq.(C.13), obtemos (usando $e^{2}=4 \pi \alpha$ )

$$
\begin{aligned}
\mathrm{d} \sigma & =\left[\left|M_{f i}^{r}\right|^{2}(2 \pi)^{4} \delta^{4}\left(P_{f}-P_{i}\right) \frac{1}{4 m \omega} \mathrm{d} \rho_{Q}\right]\left[\frac{Z^{2} \alpha}{\pi^{2}} \frac{\vec{k}_{\perp}^{2}}{\omega\left(\vec{k}_{\perp}^{2}+\frac{\omega^{2}}{\gamma^{2}}\right)^{2}}\right] \mathrm{d}^{3} p^{\prime}= \\
& =\mathrm{d} \sigma_{r} \frac{n(\vec{k})}{\omega} \mathrm{d}^{3} p^{\prime},
\end{aligned}
$$

onde definimos

$$
n(\vec{k})=\frac{Z^{2} \alpha}{\pi^{2}} \frac{\vec{k}_{\perp}^{2}}{\left(\vec{k}_{\perp}^{2}+\frac{\omega^{2}}{\gamma^{2}}\right)^{2}} .
$$

Na Eq.(C.18), d $\sigma_{r}$ é a seção de choque para o processo (a), resultante de uma colisão entre um fóton real e uma partícula em repouso, onde um sistema de partículas com momentum $Q$ é formado ; d $\sigma$ se refere ao processo (b) de formação do mesmo sistema de partículas $Q$ quando uma partícula rápida (de massa $M$ ) colide com a mesma partícula de massa $m$ em repouso do caso anterior, perdendo momentum $\vec{p}-\vec{p}^{\prime}=\vec{k}$. O fator $n(\vec{k})$ na Eq.(C.18) pode ser interpretado como a densidade de número de fótons equivalentes ao campo eletromagnético da partícula rápida de massa $M$ no espaço de $\vec{k}$.

A integração em $\mathrm{d}^{3} p^{\prime}$ é equivalente à integração em $\mathrm{d}^{3} k=\mathrm{d} k_{x} \mathrm{~d}^{2} \vec{k}_{\perp} \approx \mathrm{d}_{\omega} \mathrm{d}^{2} \vec{k}_{\perp}$ onde usamos $\omega=v k_{x}, v \approx 1$. Logo

$$
\begin{aligned}
\mathrm{d} \sigma & =\mathrm{d} \sigma_{r} \frac{n(\vec{k})}{\omega} \mathrm{d} \omega \mathrm{d}^{2} \vec{k}_{\perp} \\
& =\mathrm{d} \sigma_{r}\left(n(\vec{k}) 2 \pi\left|\vec{k}_{\perp}\right| \mathrm{d}\left|\vec{k}_{\perp}\right|\right) \frac{\mathrm{d} \omega}{\omega} \\
& =\frac{n(\omega)}{\omega} \mathrm{d} \sigma_{r} \mathrm{~d} \omega,
\end{aligned}
$$

que leva a

$$
\sigma=\int \mathrm{d} \omega \frac{n(\omega)}{\omega} \sigma_{\gamma}(\omega)
$$

onde definimos $\sigma_{r}=\sigma_{\gamma} \mathrm{e}$

$$
\begin{aligned}
n(\omega) & =\int n(\vec{k}) 2 \pi\left|\vec{k}_{\perp}\right| \mathrm{d}\left|\vec{k}_{\perp}\right| \\
& =\frac{2 Z^{2} \alpha}{\pi} \int \frac{\left|\vec{k}_{\perp}\right|^{3} \mathrm{~d}\left|\vec{k}_{\perp}\right|}{\left(\vec{k}_{\perp}^{2}+\frac{\omega^{2}}{\gamma^{2}}\right)^{2}} .
\end{aligned}
$$

A integral sobre $\mathrm{d}\left|\vec{k}_{\perp}\right|$ diverge logaritmicamente quando $\left|\vec{k}_{\perp}\right|$ é grande e isso nos possibilita obter o resultado em aproximação logaritmica. É assumido que não apenas o argumento do logaritmo é grande, mas ele próprio é grande. Para essa precisão, é suficiente tomar o limite de integração $\left|\left(\vec{k}_{\perp}\right)_{\text {max }}\right| \sim m$, que é o limite superior da desigualdade (C.15). A integração nos dá para a distribuição de frequência de fótons equivalentes $[62,124]$

$$
n(\omega)=\frac{2}{\pi} Z^{2} \alpha \log \left(\frac{\gamma m}{\omega}\right) .
$$


A equação acima nos dá uma importante informação em relação a distribuição de fótons equivalentes. Através dela, vemos que a distribuição de fótons é grande para pequeno $\omega$ e pequena para $\omega$ grande.

Na Eq.(C.21), obtivemos a equação básica que estávamos procurando. Poderíamos, por exemplo, tratar uma colisão próton-próton considerando que um próton atua como fonte de fótons, os quais irão colidir com o próton alvo. Se conhecemos a seção de choque fótonpróton para a produção de um certo estado final $X$ bem como o fluxo de fótons equivalentes devido a um próton ultra-relativístico, então podemos facilmente calcular a seção se choque para a produção do mesmo estado final $X$ devido á colisão próton-próton. A Eq.(C.21) pode ainda ser generalizada para o caso em que as duas partículas atuam como fontes de fótons os quais interagem entre si, de forma que [63]

$$
\sigma=\int \mathrm{d} \omega_{1} \mathrm{~d} \omega_{2} \frac{n\left(\omega_{1}\right)}{\omega_{1}} \frac{n\left(\omega_{2}\right)}{\omega_{2}} \sigma_{\gamma \gamma}\left(\omega_{1}, \omega_{2}\right)
$$

onde $\sigma_{\gamma \gamma}$ é a seção de choque de interação fóton-fóton, que nos possibilita estudar a produção de um estado final $X$ devido a colisão fóton-fóton.

\section{C.2 Espectro de Fótons Equivalentes Dependente do Parâmetro de Impacto}

Em alguns casos, é necessário o conhecimento da dependência que o fluxo de fótons equivalentes possui nos parâmetros de impacto. A inclusão da dependência de $b$ no fluxo de fótons pode ser feita naturalmente seguindo uma derivação semiclássica [66]. Aqui, entretanto, seguiremos a Ref.[125].

A seção de choque para a produção de uma partícula $X$ devido a interação ultraperiférica de dois hádrons é dada por

$$
\sigma_{A_{1} A_{2} \rightarrow A_{1} A_{2} X}^{W W}(\sqrt{s})=\iint \mathrm{d} \omega_{1} \mathrm{~d} \omega_{2} \frac{n_{A_{1}}\left(\omega_{1}\right)}{\omega_{1}} \frac{n_{A_{2}}\left(\omega_{2}\right)}{\omega_{2}} \sigma_{\gamma \gamma \rightarrow X}\left(\omega_{1}, \omega_{2}\right)
$$

onde $n(\omega)$ é o espectro de fótons equivalentes dado por

$$
\frac{n(\omega)}{\omega}=\frac{4 Z^{2} \alpha}{\omega} \int \frac{\mathrm{d}^{2} k_{\perp}}{(2 \pi)^{2}}\left(\frac{F\left(\vec{k}_{\perp}^{2}+\omega^{2} / \gamma^{2}\right)}{\vec{k}_{\perp}^{2}+\omega^{2} / \gamma^{2}}\right)^{2}\left|\vec{k}_{\perp}\right|^{2} .
$$

O espectro de fótons equivalentes dependente de $\mathbf{b}$, denotado por $N(\omega, b)$, é definido de forma que

$$
\frac{n(\omega)}{\omega}=\int \mathrm{d}^{2} b N(\omega, b)
$$

e pode ser escrito como

$$
N(\omega, b)=\frac{4 Z^{2} \alpha}{\omega}\left|\int \frac{\mathrm{d}^{2} k_{\perp}}{(2 \pi)^{2}} \vec{k}_{\perp} \frac{F\left(\vec{k}_{\perp}^{2}+\omega^{2} / \gamma^{2}\right)}{\vec{k}_{\perp}^{2}+\omega^{2} / \gamma^{2}} e^{-i \vec{b} \cdot \vec{k}_{\perp}}\right|^{2}
$$


uma vez que a integração em $\mathrm{d}^{2} \vec{b}$ leva a equação acima à Eq.(C.26).

Consideremos agora o caso onde exista uma simetria no ângulo azimutal. A integral em $k_{\perp}$ da Eq.(C.28) pode ser escrita como

$$
\begin{aligned}
\int \frac{\mathrm{d}^{2} k_{\perp}}{(2 \pi)^{2}} \vec{k}_{\perp} \frac{F\left(\vec{k}_{\perp}^{2}+\omega^{2} / \gamma^{2}\right)}{\vec{k}_{\perp}^{2}+\omega^{2} / \gamma^{2}} e^{-i \vec{b} \cdot \vec{k}_{\perp}} & =\int \frac{\mathrm{d} \varphi k_{\perp} \mathrm{d} k_{\perp}}{(2 \pi)^{2}} \frac{F\left(\vec{k}_{\perp}^{2}+\omega^{2} / \gamma^{2}\right)}{\vec{k}_{\perp}^{2}+\omega^{2} / \gamma^{2}} \vec{k}_{\perp} e^{-i \vec{b} \cdot \vec{k}_{\perp}} \\
& =\int \frac{\mathrm{d} \varphi k_{\perp} \mathrm{d} k_{\perp}}{(2 \pi)^{2}} \frac{F\left(\vec{k}_{\perp}^{2}+\omega^{2} / \gamma^{2}\right)}{\vec{k}_{\perp}^{2}+\omega^{2} / \gamma^{2}} \vec{k}_{\perp} e^{-i b k_{\perp} \cos \varphi}
\end{aligned}
$$

Vamos reescrever o vetor $\vec{k}_{\perp}$ de uma forma mais adequada. Primeiramente, notamos que

$$
\nabla_{b} e^{-i \vec{b} \cdot \vec{k}_{\perp}}=-i \vec{k}_{\perp} e^{-i \vec{b} \cdot \vec{k}_{\perp}}
$$

Então a integral em $k_{\perp}$ fica

$$
\begin{aligned}
I & =-i \int \frac{k_{\perp} \mathrm{d} k_{\perp}}{(2 \pi)^{2}} \frac{F\left(\vec{k}_{\perp}^{2}+\omega^{2} / \gamma^{2}\right)}{\vec{k}_{\perp}^{2}+\omega^{2} / \gamma^{2}} \nabla_{b}\left[\int_{0}^{2 \pi} e^{-i b k_{\perp} \cos \varphi} \mathrm{d} \varphi\right] \\
& =-i \int \frac{k_{\perp} \mathrm{d} k_{\perp}}{(2 \pi)^{2}} \frac{F\left(\vec{k}_{\perp}^{2}+\omega^{2} / \gamma^{2}\right)}{\vec{k}_{\perp}^{2}+\omega^{2} / \gamma^{2}} 2 \pi \nabla_{b} J_{0}\left(b k_{\perp}\right)
\end{aligned}
$$

onde usamos

$$
\int_{0}^{2 \pi} e^{-i b k_{\perp} \cos \varphi} \mathrm{d} \varphi=2 \pi J_{0}\left(b k_{\perp}\right)
$$

Vamos agora aplicar o gradiente em coordenadas polares na $J_{0}\left(b k_{\perp}\right)$ :

$$
\begin{aligned}
\nabla_{b} J_{0}\left(b k_{\perp}\right) & =\hat{e}_{b} \frac{\partial J_{0}\left(b k_{\perp}\right)}{\partial b}+\frac{1}{b} \hat{e}_{\varphi} \frac{\partial J_{0}\left(b k_{\perp}\right)}{\partial \varphi} \\
& =-\hat{e}_{b} k_{\perp} J_{1}\left(b k_{\perp}\right)
\end{aligned}
$$

onde usamos

$$
J_{0}^{\prime}(x)=-J_{1}(x)
$$

Levando a Eq.(C.31) na Eq.(C.29) e o resultado disso na Eq.(C.28), obtemos

$$
N(\omega, b)=\frac{Z^{2} \alpha}{\pi^{2} \omega}\left|\int_{0}^{\infty} \mathrm{d} k_{\perp} k_{\perp}^{2} \frac{F\left(\vec{k}_{\perp}^{2}+\omega^{2} / \gamma^{2}\right)}{\vec{k}_{\perp}^{2}+\omega^{2} / \gamma^{2}} J_{1}\left(b k_{\perp}\right)\right|^{2}
$$

onde usamos o fato de que $\hat{e}_{b} \cdot \hat{e}_{b}=1$.

Por fim, fazendo a seguinte troca de variáveis

$$
k=\frac{u}{b},
$$


obtemos para a integral em $k_{\perp}$

$$
\begin{aligned}
\int_{0}^{\infty} \frac{\mathrm{d} u}{b} \frac{u^{2}}{b^{2}} \frac{F\left[\frac{u^{2}}{b^{2}}+\frac{\omega^{2}}{\gamma^{2}}\right]}{\left[\frac{u^{2}}{b^{2}}+\frac{\omega^{2}}{\gamma^{2}}\right]} J_{1}(u) & =\int_{0}^{\infty} \mathrm{d} u u^{2} J_{1}(u) \frac{b^{2}}{b^{3}} \frac{F\left[\frac{u^{2}+\frac{b^{2} \omega^{2}}{\gamma^{2}}}{b^{2}}\right]}{\left[u^{2}+\frac{b^{2} \omega^{2}}{\gamma^{2}}\right]} J_{1}(u) \\
& =\frac{1}{b} \int_{0}^{\infty} \mathrm{d} u u^{2} J_{1}(u) \frac{F\left[\frac{u^{2}+\frac{b^{2} \omega^{2}}{\gamma^{2}}}{b^{2}}\right]}{\left[u^{2}+\frac{b^{2} \omega^{2}}{\gamma^{2}}\right]} J_{1}(u)
\end{aligned}
$$

Levando a equação acima na Eq.(C.33), obtemos

$$
N(\omega, b)=\frac{Z^{2} \alpha}{\pi^{2} \omega b^{2}}\left|\int_{0}^{\infty} \mathrm{d} u u^{2} J_{1}(u) \frac{F\left[\frac{u^{2}+\left(\frac{b \omega}{\gamma}\right)^{2}}{b^{2}}\right]}{\left[u^{2}+\left(\frac{b \omega}{\gamma}\right)^{2}\right]}\right|^{2} .
$$

\section{C.2.1 Fatores de Forma}

\section{Núcleo}

Vamos trabalhar com a integral da Eq.(C.36). Para o fator de forma de monopolo no caso nuclear, temos

$$
F(q)=\frac{\Lambda^{2}}{\Lambda^{2}+q^{2}}
$$

Reescrevendo $F(q)$ na forma da Eq.(C.36) e tomando $x=b \omega / \gamma$, obtemos

$$
F(q)=\frac{b^{2} \Lambda^{2}}{b^{2} \Lambda^{2}+x^{2}+u^{2}}
$$

Podemos escrever

$$
\frac{F\left(\sqrt{\frac{\left(\frac{b \omega}{\gamma}\right)^{2}+u^{2}}{b^{2}}}\right)}{x^{2}+u^{2}}=\frac{1}{x^{2}+u^{2}}-\frac{1}{b^{2} \Lambda^{2}+x^{2}+u^{2}}
$$

que é parte do integrando da integral em $u$.

Usando a fórmula [126]

$$
\int_{0}^{\infty} \frac{u^{\nu+1} J_{\nu}(a u) \mathrm{d} u}{\left(u^{2}+x^{2}\right)^{\mu+1}}=\frac{a^{\mu} x^{\nu-\mu}}{2^{\mu} \Gamma(\mu+1)} K_{\nu-\mu}(a u)
$$

válido para $a>0 ; \operatorname{Rex}>0$ e $-1<\operatorname{Re\nu }<\operatorname{Re} \mu+3 / 2$. Tomando em (C.40) $a=1, \nu=$ $1, \mu=0$, obtemos para a integral da Eq.(C.36) (primeiro termo de (C.39))

$$
\int_{0}^{\infty} \frac{u^{2} J_{1}(u) \mathrm{d} u}{\left(u^{2}+x^{2}\right)}=x K_{1}(x)
$$


Fazendo algo semelhante para o segundo termo de (C.39), obtemos

$$
N(\omega, b)=\frac{Z^{2} \alpha_{e m}}{\pi^{2} \omega}\left[\frac{\omega}{\gamma} K_{1}\left(\frac{b \omega}{\gamma}\right)-\sqrt{\left(\frac{\omega^{2}}{\gamma^{2}}+\Lambda^{2}\right)} K_{1}\left(b \sqrt{\frac{\omega^{2}}{\gamma^{2}}+\Lambda^{2}}\right)\right]^{2}
$$

que é o espectro de fótons equivalentes devido ao núcleo.

\section{Próton}

O fator de forma (de dipolo) do próton é

$$
F(q)=\frac{\Lambda^{4}}{\left(\Lambda^{2}+q^{2}\right)^{2}}
$$

Tomando $x=b \omega / \gamma$, temos

$$
\begin{aligned}
& F\left(\sqrt{\frac{x^{2}+u^{2}}{b^{2}}}\right)=\frac{\Lambda^{4}}{\left(\Lambda^{2}+\frac{x^{2}+u^{2}}{b^{2}}\right)^{2}} \\
= & \frac{b^{4} \Lambda^{4}}{\left(b^{2} \Lambda^{2}+x^{2}+u^{2}\right)^{2}}=\frac{b^{4} \Lambda^{4}}{\left(z^{2}+u^{2}\right)^{2}}
\end{aligned}
$$

onde definimos $z^{2}=b^{2} \Lambda^{2}+x^{2}=b^{2}\left(\Lambda^{2}+\frac{\omega^{2}}{\gamma^{2}}\right)$. Então

$$
\begin{gathered}
\frac{F\left(\sqrt{\frac{x^{2}+u^{2}}{b^{2}}}\right)}{x^{2}+u^{2}}=\frac{b^{4} \Lambda^{4}}{\left(b^{2} \Lambda^{2}+x^{2}+u^{2}\right)^{2}} \frac{1}{x^{2}+u^{2}} \\
=-\frac{1}{x^{2}+b^{2} \Lambda^{2}+u^{2}}-\frac{b^{2} \Lambda^{2}}{\left(x^{2}+b^{2} \Lambda^{2}+u^{2}\right)^{2}}+\frac{1}{x^{2}+u^{2}} .
\end{gathered}
$$

Temos que fazer 3 integrais referentes à Eq.(C.36):

(i) tomando $\nu=1, \mu=0, a=1$ :

$$
-\int_{0}^{\infty} \frac{u^{2} J_{1}(u) \mathrm{d} u}{\left(u^{2}+z^{2}\right)}=-z K_{1}(z)
$$

(ii) tomando $\nu=1, \mu=1, a=1$ :

$$
-b^{2} \Lambda^{2} \int_{0}^{\infty} \frac{u^{2} J_{1}(u) \mathrm{d} u}{\left(u^{2}+z^{2}\right)^{2}}=-\frac{b^{2} \Lambda^{2}}{2} K_{0}(z)
$$

(iii) tomando $\nu=1, \mu=0, a=1$ :

$$
\int_{0}^{\infty} \frac{u^{2} J_{1}(u) \mathrm{d} u}{\left(u^{2}+x^{2}\right)}=x K_{1}(x)
$$


Dessa forma, o fluxo do próton é

$$
N(\omega, b)=\frac{\alpha_{e m}}{\pi^{2}} \frac{1}{b^{2} \omega}\left[-z K_{1}(z)-\frac{b^{2} \Lambda^{2}}{2} K_{0}(z)+x K_{1}(x)\right]^{2}
$$

lembrando que

$$
x=\frac{b \omega}{\gamma}
$$

e

$$
z=b \sqrt{\Lambda^{2}+\frac{\omega^{2}}{\gamma^{2}}}
$$

Por fim, o fluxo pode ser reescrito como

$$
\begin{array}{r}
N(\omega, b)=\frac{\alpha_{e m}}{\pi^{2}} \frac{1}{\omega}\left[\frac{\omega}{\gamma} K_{1}\left(\frac{b \omega}{\gamma}\right)-\sqrt{\left(\Lambda^{2}+\frac{\omega^{2}}{\gamma^{2}}\right)} K_{1}\left(b \sqrt{\left(\Lambda^{2}+\frac{\omega^{2}}{\gamma^{2}}\right)}\right)\right. \\
\left.-\frac{b \Lambda^{2}}{2} K_{0}\left(b \sqrt{\left(\Lambda^{2}+\frac{\omega^{2}}{\gamma^{2}}\right)}\right)\right]^{2}
\end{array}
$$

que é o espectro de fótons equivalentes devido ao próton. 


\section{Bibliografia}

[1] V. P. Goncalves, B. D. Moreira, and F. S. Navarra. Investigation of diffractive photoproduction of $J / \Psi$ in hadronic collisions. Phys.Rev., C90(1):015203, 2014.

[2] V. P. Goncalves, B. D. Moreira, and F. S. Navarra. Exclusive $\Upsilon$ photoproduction in hadronic collisions at CERN LHC energies. Phys.Lett., B742:172-177, 2015.

[3] F. Carvalho, V. P. Goncalves, B. D. Moreira, and F. S. Navarra. Double vector meson production in the International Linear Collider. Eur. Phys. J., C75(8):392, 2015.

[4] V. P. Goncalves, B. D. Moreira, and F. S. Navarra. Double vector meson production in $\gamma \gamma$ interactions at hadronic colliders. Eur. Phys. J., C76(3):103, 2016.

[5] V. P. Goncalves, B. D. Moreira, and F. S. Navarra. Double vector meson production in photon-hadron interactions at hadronic colliders. Eur. Phys. J., C76(7):388, 2016.

[6] V. P. Goncalves, B. D. Moreira, and F. S. Navarra. Exclusive heavy vector meson photoproduction in hadronic collisions at the LHC: predictions of the Color Glass Condensate model for Run 2 energies, arXiv:1612.06254, 2016.

[7] D. J. Gross and F. Wilczek. Ultraviolet Behavior of Nonabelian Gauge Theories. Phys.Rev.Lett., 30:1343-1346, 1973.

[8] H. D. Politzer. Reliable Perturbative Results for Strong Interactions? Phys.Rev.Lett., 30:1346-1349, 1973.

[9] Y. V. Kovchegov and E. Levin. Quantum Chromodynamics at High Energy. Cambridge University Press, Cambridge, 2012.

[10] M. E. Peskin and D. V. Schroeder. An Introduction To Quantum Field Theory (Frontiers in Physics). Westview Press, 1995.

[11] R. Devenish and A. Cooper-Sarkar. Deep Inelastic Scattering. Oxford University Press, New York, 2004.

[12] J. D. Bjorken. Asymptotic Sum Rules at Infinite Momentum. Phys.Rev., 179:15471553, 1969.

[13] P. Marage. Hadronic structure, low x physics and diffraction, arXiv:9911426, 1999.

[14] K. Hagiwara et al. Review of particle physics. Particle Data Group. Phys.Rev., D66:010001, 2002. 
[15] Y. L. Dokshitzer. Calculation of the Structure Functions for Deep Inelastic Scattering and e+ e- Annihilation by Perturbation Theory in Quantum Chromodynamics. Sov.Phys.JETP, 46:641-653, 1977.

[16] V. N. Gribov and L. N. Lipatov. Deep inelastic e p scattering in perturbation theory. Sov.J.Nucl.Phys., 15:438-450, 1972.

[17] G. Altarelli and G. Parisi. Asymptotic Freedom in Parton Language. Nucl.Phys., B126:298, 1977.

[18] V. Barone and E. Predazzi. High-Energy Particle Diffraction. Springer, Berlin, 2002.

[19] C. G. J. Callan and D. J. Gross. High-energy electroproduction and the constitution of the electric current. Phys.Rev.Lett., 22:156-159, 1969.

[20] C. Adloff et al. Deep inelastic inclusive e p scattering at low x and a determination of alpha(s). Eur. Phys. J., C21:33-61, 2001.

[21] S. Chekanov et al. A ZEUS next-to-leading-order QCD analysis of data on deep inelastic scattering. Phys. Rev., D67:012007, 2003.

[22] E. A. Kuraev, L. N. Lipatov, and V. S. Fadin. The Pomeranchuk Singularity in Nonabelian Gauge Theories. Sov.Phys.JETP, 45:199-204, 1977.

[23] I. I. Balitsky and L. N. Lipatov. The Pomeranchuk Singularity in Quantum Chromodynamics. Sov.J.Nucl.Phys., 28:822-829, 1978.

[24] L. V. Gribov, E. M. Levin, and M. G. Ryskin. Semihard Processes in QCD. Phys.Rept., 100:1-150, 1983.

[25] I. Balitsky. Operator expansion for high-energy scattering. Nucl.Phys., B463:99-160, 1996.

[26] Y. V. Kovchegov. Small x F(2) structure function of a nucleus including multiple pomeron exchanges. Phys.Rev., D60:034008, 1999.

[27] N. Nikolaev and B. G. Zakharov. Color transparency and scaling properties of nuclear shadowing in deep inelastic scattering. Z. Phys., C49:607-618, 1991.

[28] N. N. Nikolaev and B. G. Zakharov. Pomeron structure function and diffraction dissociation of virtual photons in perturbative QCD. Z. Phys., C53:331-346, 1992.

[29] N. N. Nikolaev and B. G. Zakharov. The Triple pomeron regime and the structure function of the pomeron in the diffractive deep inelastic scattering at very small $\mathrm{x} . Z$. Phys., C64:631-652, 1994.

[30] A. H. Mueller. Soft gluons in the infinite momentum wave function and the BFKL pomeron. Nucl.Phys., B415:373-385, 1994. 
[31] H. Kowalski, L. Motyka, and G. Watt. Exclusive diffractive processes at HERA within the dipole picture. Phys.Rev., D74:074016, 2006.

[32] S. Munier, A. M. Stasto, and A. H. Mueller. Impact parameter dependent S matrix for dipole proton scattering from diffractive meson electroproduction. Nucl.Phys., B603:427-445, 2001.

[33] M. Froissart. Asymptotic behavior and subtractions in the Mandelstam representation. Phys. Rev., 123:1053-1057, 1961.

[34] A. Martin. Unitarity and high-energy behavior of scattering amplitudes. Phys. Rev., 129:1432-1436, 1963.

[35] A. H. Mueller and J. Qiu. Gluon Recombination and Shadowing at Small Values of x. Nucl.Phys., B268:427, 1986.

[36] Y. V. Kovchegov. Brief Review of Saturation Physics. Acta Phys. Polon., B45(12):2241-2256, 2014.

[37] L. D. McLerran. What is the evidence for the color glass condensate? In Structure and dynamics of elementary matter. Proceedings, NATO Advanced Study Institute, Camyuva-Kemer, Turkey, September 22-October 2, 2003, pages 183-205, 2004.

[38] L. D. McLerran and R. Venugopalan. Computing quark and gluon distribution functions for very large nuclei. Phys. Rev., D49:2233-2241, 1994.

[39] L. D. McLerran and R. Venugopalan. Gluon distribution functions for very large nuclei at small transverse momentum. Phys. Rev., D49:3352-3355, 1994.

[40] L. D. McLerran and R. Venugopalan. Green's functions in the color field of a large nucleus. Phys. Rev., D50:2225-2233, 1994.

[41] E. Iancu. QCD in heavy ion collisions, arXiv:1205.0579,2014.

[42] J. Jalilian-Marian and Y. V. Kovchegov. Saturation physics and deuteron-Gold collisions at RHIC. Prog.Part.Nucl.Phys., 56:104-231, 2006.

[43] T. Lappi. FYSH560 High Energy Scattering in QCD. University of Jyväskylä, Departmanent of Physics, Lecture notes, 2011.

[44] H. Mäntysaari. Balitsky-Kovchegov Equation. University of Jyväskylä, Departmanent of Physics, Tese de doutorado, 2011.

[45] K. J. Golec-Biernat and M. Wusthoff. Saturation effects in deep inelastic scattering at low $Q^{2}$ and its implications on diffraction. Phys.Rev., D59:014017, 1998.

[46] K. J. Golec-Biernat and M. Wusthoff. Saturation in diffractive deep inelastic scattering. Phys.Rev., D60:114023, 1999. 
[47] M. Kozlov, A. Shoshi, and W. Xiang. On possible implications of gluon number fluctuations in DIS data. JHEP, 0710:020, 2007.

[48] E. Iancu, K. Itakura, and S. Munier. Saturation and BFKL dynamics in the HERA data at small x. Phys.Lett., B590:199-208, 2004.

[49] G. Soyez. Saturation QCD predictions with heavy quarks at HERA. Phys.Lett., B655:32-38, 2007.

[50] A. H. Rezaeian and I. Schmidt. Impact-parameter dependent Color Glass Condensate dipole model and new combined HERA data. Phys.Rev., D88:074016, 2013.

[51] J. L. Albacete, N. Armesto, J. G. Milhano, and Carlos A. Salgado. Non-linear QCD meets data: A Global analysis of lepton-proton scattering with running coupling BK evolution. Phys.Rev., D80:034031, 2009.

[52] A. M. Stasto, K. J. Golec-Biernat, and J. Kwiecinski. Geometric scaling for the total $\gamma^{*} p$ cross-section in the low x region. Phys. Rev. Lett., 86:596-599, 2001.

[53] H. Abramowicz and A. Caldwell. HERA collider physics. Rev. Mod. Phys., 71:12751410, 1999.

[54] E. Levin and A. H. Rezaeian. Gluon saturation and energy dependence of hadron multiplicity in pp and AA collisions at the LHC. Phys. Rev., D83:114001, 2011.

[55] V. Khachatryan et al. Transverse-momentum and pseudorapidity distributions of charged hadrons in $p p$ collisions at $\sqrt{s}=7 \mathrm{TeV}$. Phys. Rev. Lett., 105:022002, 2010.

[56] E. Fermi. On the Theory of the impact between atoms and electrically charged particles. Z.Phys., 29:315-327, 1924.

[57] C. F. von Weizsacker. Radiation emitted in collisions of very fast electrons. Z.Phys., 88:612-625, 1934.

[58] E. J. Williams. Correlation of Certain Collisions Problems with Radiation Theory. Mat.-Fys. Meddel, 13:4, 1935.

[59] C. A. Bertulani, S.r R. Klein, and J. Nystrand. Physics of ultra-peripheral nuclear collisions. Ann.Rev.Nucl.Part.Sci., 55:271-310, 2005.

[60] A. J. Baltz. The Physics of Ultraperipheral Collisions at the LHC. Phys. Rept., 458:1-171, 2008.

[61] G. Baur et al. Hot topics in ultraperipheral ion collisions. In Electromagnetic probes of fundamental physics. Proceedings, Workshop, Erice, Italy, October 16-21, 2001, pages 235-241, 2002.

[62] V. B. Berestetskii, E. M. Lifshitz, and L. P. Pitaevskii. Quantum Electrodynamics. Pergamon Press, New York, 1982. 
[63] V. M. Budnev, I. F. Ginzburg, G. V. Meledin, and V. G. Serbo. The Two photon particle production mechanism. Physical problems. Applications. Equivalent photon approximation. Phys. Rept., 15:181-281, 1975.

[64] J. Nystrand. Electromagnetic interactions in nucleus-nucleus and proton-proton collisions. Nucl.Phys., A752:470-479, 2005.

[65] M. Drees and D. Zeppenfeld. Production of Supersymmetric Particles in Elastic ep Collisions. Phys. Rev., D39:2536, 1989.

[66] J. D. Jackson. New York, NY, 3rd ed. edition.

[67] C. A. Bertulani. Probing Two-Photon Decay Widths of Mesons at energies available at the CERN LHC. Phys. Rev., C79:047901, 2009.

[68] B. D. Moreira, C. A. Bertulani, V. P. Goncalves, and F. S. Navarra. Production of exotic charmonium in $\gamma \gamma$ interactions at hadronic colliders. Phys. Rev., D94:094024, 2016.

[69] M. Klusek-Gawenda and A. Szczurek. Exclusive muon-pair productions in ultrarelativistic heavy-ion collisions - realistic nucleus charge form factor and differential distributions. Phys. Rev., C82:014904, 2010.

[70] J. A. Crittenden. Exclusive production of neutral vector mesons at the electron proton collider HERA. 1997.

[71] J. L. Abelleira Fernandez et al. A Large Hadron Electron Collider at CERN: Report on the Physics and Design Concepts for Machine and Detector. J. Phys., G39:075001, 2012.

[72] C. Marquet. Open questions in QCD at high parton density. Nucl. Phys., A904905:294c-301c, 2013.

[73] C. Adler et al. Coherent $\rho^{0}$ production in ultraperipheral heavy ion collisions. Phys. Rev. Lett., 89:272302, 2002.

[74] B. I. Abelev et al. $\rho^{0}$ photoproduction in ultraperipheral relativistic heavy ion collisions at $\sqrt{s_{N N}}=200 \mathrm{GeV}$. Phys. Rev., C77:034910, 2008.

[75] S. Klein and J. Nystrand. Exclusive vector meson production in relativistic heavy ion collisions. Phys. Rev., C60:014903, 1999.

[76] V. P. Goncalves and C. A. Bertulani. Peripheral heavy ion collisions as a probe of the nuclear gluon distribution. Phys. Rev., C65:054905, 2002.

[77] L. Frankfurt, M. Strikman, and M. Zhalov. Fading out of $J / \psi$ color transparency in high-energy heavy ion peripheral collisions. Phys. Lett., B540:220-226, 2002. 
[78] V. P. Goncalves and M. V. T. Machado. The QCD pomeron in ultraperipheral heavy ion collisions. IV. Photonuclear production of vector mesons. Eur. Phys. J., C40:519$529,2005$.

[79] L. Frankfurt, M. Strikman, and M. Zhalov. Predictions of the generalized Glauber model for the coherent rho production at RHIC and the STAR data. Phys. Rev., C67:034901, 2003.

[80] S. Chatrchyan et al. Exclusive photon-photon production of muon pairs in protonproton collisions at $\sqrt{s}=7 \mathrm{TeV}$. JHEP, 01:052, 2012.

[81] S. Chatrchyan et al. Study of exclusive two-photon production of $W^{+} W^{-}$in $p p$ collisions at $\sqrt{s}=7 \mathrm{TeV}$ and constraints on anomalous quartic gauge couplings. JHEP, 07:116, 2013.

[82] B. Abelev et al. Coherent $J / \psi$ photoproduction in ultra-peripheral $\mathrm{Pb}-\mathrm{Pb}$ collisions at $\sqrt{s_{N N}}=2.76 \mathrm{TeV}$. Phys.Lett., B718:1273-1283, 2013.

[83] K. Akiba et al. LHC Forward Physics. J. Phys., G43:110201, 2016.

[84] G. G. da Silveira. Exclusive processes in pp collisions in CMS. In Proceedings, 21st International Workshop on Deep-Inelastic Scattering and Related Subjects (DIS 2013): Marseilles, France, April 22-26, 2013, 2013.

[85] J. Bartels, K. J. Golec-Biernat, and Krisztian Peters. On the dipole picture in the nonforward direction. Acta Phys. Polon., B34:3051-3068, 2003.

[86] A. G. Shuvaev, K. J. Golec-Biernat, A. D. Martin, and M. G. Ryskin. Off diagonal distributions fixed by diagonal partons at small $\mathrm{x}$ and xi. Phys. Rev., D60:014015, 1999.

[87] N. Armesto. A Simple model for nuclear structure functions at small $\mathrm{x}$ in the dipole picture. Eur.Phys.J., C26:35-43, 2002.

[88] K. Tuchin. Coherent and incoherent diffractive hadron production in pA collisions and gluon saturation. Phys. Rev., C79:055206, 2009.

[89] T. Lappi and H. Mantysaari. $J / \psi$ production in ultraperipheral $\mathrm{Pb}+\mathrm{Pb}$ and $p+\mathrm{Pb}$ collisions at energies available at the CERN Large Hadron Collider. Phys.Rev., C87(3):032201, 2013.

[90] B.Z. Kopeliovich, J. Nemchik, A. Schafer, and A.V. Tarasov. Color transparency versus quantum coherence in electroproduction of vector mesons off nuclei. Phys.Rev., C65:035201, 2002.

[91] E. Andrade-II, I. Gonzlez, A. Deppman, and C. A. Bertulani. Evidence of subnucleonic degrees of freedom in $\mathrm{J} / \psi$ photoproduction in ultraperipheral collisions at the CERN Large Hadron Collider. Phys. Rev., C92:064903, 2015. 
[92] V. Guzey, M. Strikman, and M. Zhalov. Disentangling coherent and incoherent quasielastic $J / \psi$ photoproduction on nuclei by neutron tagging in ultraperipheral ion collisions at the LHC. Eur. Phys. J., C74(7):2942, 2014.

[93] R Aaij et al. Exclusive $J / \psi$ and $\psi(2 \mathrm{~S})$ production in pp collisions at $\sqrt{s}=7 \mathrm{TeV}$. J.Phys., G40:045001, 2013.

[94] R. Aaij et al. Updated measurements of exclusive $J / \psi$ and $\psi(2 \mathrm{~S})$ production crosssections in pp collisions at $\sqrt{s}=7 \mathrm{TeV}$. J.Phys., G41:055002, 2014.

[95] E. Abbas et al. Charmonium and $e^{+} e^{-}$pair photoproduction at mid-rapidity in ultraperipheral $\mathrm{Pb}-\mathrm{Pb}$ collisions at $\sqrt{s_{\mathrm{NN}}}=2.76 \mathrm{TeV}$. Eur.Phys.J., C73(11):2617, 2013.

[96] S. Chekanov et al. Exclusive photoproduction of $J / \psi$ mesons at HERA. Eur.Phys.J., C24:345-360, 2002.

[97] A. Aktas et al. Elastic $J / \psi$ production at HERA. Eur.Phys.J., C46:585-603, 2006.

[98] C. Alexa et al. Elastic and Proton-Dissociative Photoproduction of $J / \psi$ Mesons at HERA. Eur.Phys.J., C73(6):2466, 2013.

[99] G. Watt and H. Kowalski. Impact parameter dependent colour glass condensate dipole model. Phys.Rev., D78:014016, 2008.

[100] B. Abelev et al. Exclusive $\mathrm{J} / \psi$ photoproduction off protons in ultra-peripheral p-Pb collisions at $\sqrt{s_{\mathrm{NN}}}=5.02 \mathrm{TeV}$. Phys. Rev. Lett., 113(23):232504, 2014.

[101] A. C. Caldwell and M. S. Soares. Vector meson production in the Golec-Biernat Wusthoff model. Nucl.Phys., A696:125-137, 2001.

[102] B. E. Cox, J. R. Forshaw, and R. Sandapen. Diffractive $\Upsilon$ production at the LHC. JHEP, 0906:034, 2009.

[103] J. Breitweg et al. Measurement of elastic $\Upsilon$ photoproduction at HERA. Phys.Lett., B437:432-444, 1998.

[104] C. Adloff et al. Elastic photoproduction of $J / \psi$ and $\Upsilon$ mesons at HERA. Phys.Lett., B483:23-35, 2000.

[105] S. Chekanov et al. Exclusive photoproduction of $\Upsilon$ mesons at HERA. Phys.Lett., B680:4-12, 2009.

[106] R. Aaij et al. Measurement of the exclusive production cross-section in pp collisions at $\sqrt{s}=7 \mathrm{TeV}$ and $8 \mathrm{TeV}$. JHEP, 09:084, 2015.

[107] G. Sampaio dos Santos and M. V. T. Machado. Exclusive photoproduction of quarkonium in proton-nucleus collisions at energies available at the CERN Large Hadron Collider. Phys. Rev., C89(2):025201, 2014. 
[108] G. Sampaio dos Santos and M. V. T. Machado. Light vector meson photoproduction in hadron-hadron and nucleus-nucleus collisions at energies available at the CERN Large Hadron Collider. Phys. Rev., C91(2):025203, 2015.

[109] G. Sampaio dos Santos and M. V. T. Machado. On theoretical uncertainty of color dipole phenomenology in the $J / \psi$ and $\Upsilon$ photoproduction in pA and AA collisions at the CERN Large Hadron Collider. J. Phys., G42(10):105001, 2015.

[110] N. Timneanu, J. Kwiecinski, and L. Motyka. Saturation model for two photon interactions at high-energies. Eur.Phys.J., C23:513-526, 2002.

[111] V.P. Goncalves and M.V.T. Machado. Dipole model for double meson production in two-photon interactions at high energies. Eur.Phys.J., C49:675-684, 2007.

[112] V. P. Goncalves, M. S. Kugeratski, E. R. Cazaroto, F. Carvalho, and F. S. Navarra. Non-linear QCD dynamics in two-photon interactions at high energies. Eur.Phys.J., C71:1779, 2011.

[113] I. F. Ginzburg, S. L. Panfil, and V. G. Serbo. The Semihard Processes $\gamma \gamma \rightarrow \psi$ X, $\gamma \gamma \rightarrow \psi \psi, \gamma \gamma \rightarrow \rho \psi$. Nucl. Phys., B296:569-581, 1988.

[114] J. Kwiecinski and L. Motyka. Diffractive $J / \psi$ production in high-energy $\gamma \gamma$ collisions as a probe of the QCD pomeron. Phys. Lett., B438:203-210, 1998.

[115] V. P. Goncalves and W. K. Sauter. Double vector meson production from the BFKL equation. Eur. Phys. J., C44:515-522, 2005.

[116] M. Klusek, W. Schafer, and A. Szczurek. Exclusive production of $\rho^{0} \rho^{0}$ pairs in $\gamma \gamma$ collisions at RHIC. Phys. Lett., B674:92-97, 2009.

[117] S. Baranov, A. Cisek, M. Klusek-Gawenda, W. Schafer, and A. Szczurek. The $\gamma \gamma \rightarrow J / \psi J / \psi$ reaction and the $J / \psi J / \psi$ pair production in exclusive ultraperipheral ultrarelativistic heavy ion collisions. Eur. Phys. J., C73(2):2335, 2013.

[118] M. Kusek-Gawenda and A. Szczurek. Double-scattering mechanism in the exclusive $\mathrm{AA} \rightarrow \mathrm{AA} \rho^{0} \rho^{0}$ reaction in ultrarelativistic collisions. Phys. Rev., C89(2):024912, 2014.

[119] H. G. Dosch, T. Gousset, G. Kulzinger, and H. J. Pirner. Vector meson leptoproduction and nonperturbative gluon fluctuations in QCD. Phys. Rev., D55:2602-2615, 1997.

[120] R. Sandapen. The Colour Dipole Model Of Diffractive Scattering. The University of Manchester, Tese de doutorado, 2002.

[121] E. Iancu, K. Itakura, and L. McLerran. Geometric scaling above the saturation scale. Nucl.Phys., A708:327-352, 2002.

[122] A. H. Mueller and D. N. Triantafyllopoulos. The Energy dependence of the saturation momentum. Nucl.Phys., B640:331-350, 2002. 
[123] E. Levin and K. Tuchin. Solution to the evolution equation for high parton density QCD. Nucl.Phys., B573:833-852, 2000.

[124] C. A. Bertulani and G. Baur. Electromagnetic Processes in Relativistic Heavy Ion Collisions. Phys.Rept., 163:299, 1988.

[125] M. Vidovic, M. Greiner, C. Best, and G. Soff. Impact parameter dependence of the electromagnetic particle production in ultrarelativistic heavy ion collisions. Phys. Rev., C47:2308-2319, 1993.

[126] M. Abramowitz and I. A. Stegun. Handbook of mathematical functions : with formulas, graphs, and mathematical tables. Dover, New York, 1965. 